


\section{Líbrare \\ OF THE}

Whew Work state Weterinarp College

AT

Cornell Unípersity 
SF 919.F92 Cornell University Library

Text-book of general therapeutics for ve

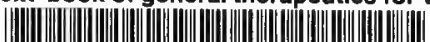

31924003359076 vet 


\section{Cornell University Library}

The original of this book is in the Cornell University Library.

There are no known copyright restrictions in the United States on the use of the text. 


\title{
TEXT-BOOK OF
}

\section{GENERAL THERAPEUTICS}

\section{FOR VETERINARIANS}

\author{
BY \\ EUGEN FRÖHNER \\ PRIVY COUNCILLOR AND PROFESSOR OF SPECIAL PATHOLOGY AND THERAPEUTICS IN THE \\ VETERINARY COLLEGE AT BERLIN
}

AUTHORIZED TRANSLATION FROM THE FOURTH REVISED

GERMAN EDITION

BY

LOUIS A. KLEIN

PROFESSOR OF PHARMACOLOGY AND VETERINARY HYGIENE IN THE SCHOOL OF VETERINAEY MEDICINE AT THE UNIVERSITY OF PENNSYLVANIA, AND DEAN OF THE FACULTY

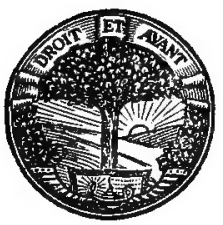

PHILADELPHIA \& LONDON J. B. LIPPINCOTT COMPANY 


$$
\text { MYS } 58 .
$$

Copyright, 1914, by J. B. LIPPINCoxt CoMPanY

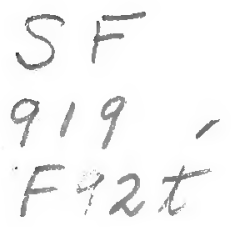

$$
\begin{aligned}
& \text { NEW YORE STATE } \\
& \text { VTERINARY COLLEGE } \\
& \text { LIUARY }
\end{aligned}
$$

PRINTED BY J. B. IIPPINCOTT COMPANY AT THE WASHINGTON SQUARE PRESS

PEILADELPEIA, U. S. A. 


\section{TRANSLATOR'S PREFACE}

THE popularity of Fröhner's General Therapeutics among veterinarians to whom it is accessible in German and the lack of a similar work in English suggested this translation, which it is hoped will prove equally as serviceable to American and English veterinarians. A few additions which have been made to the text, most of them of an explanatory character, have been placed between brackets ([l]). The German regulations governing disinfection for the different infectious diseases have been included in the translation; while they do not have the force of law in this country, the methods they describe, with some allowance for differences in conditions, are also applicable here. The etymological footnotes explaining the derivation of the terms referring to therapeutic action have been omitted. In the descriptions of the therapeutic uses of the individual drugs, the names used in the United States Pharmacopœia and in the United States Dispensatory have been employed, and these have been followed, wherever it seemed desirable, by the common or English name. Nonofficial drugs are indicated by an asterisk $\left({ }^{*}\right)$. Doses are given in the metric system, as in the original, with the equivalent in the apothecaries' system.

LOUIS A. KLEIN.

Philadelphia, July, 1914. 



\section{PREFACE TO THE FOURTH EDITION}

Is the new edition of General Therapeutics the chapters on chemotherapy, protective vaccination, and disinfection have been rewritten. The "therapia sterilisans magna" of Ehrlich has become of great importance to veterinarians, although at the beginning of its introduction the judgment concerning it was too optimistic (contagious pneumonia!). Considerable development has also occurred in the last ten years in the field of protective vaccination. The more complete vaccination statistics now available make possible a more exact judgment concerning the value or worthlessness of the different immune sera. More recent observations have compelled a partial modification of our views in regard to disinfection; this is especially true in respect to preliminary disinfection and its relation to cleaning. The new German veterinary sanitary law has been included in the revision. The reader will also find numerous changes and improvements in other chapters.

E. Fröhner.

BerLin. 



\section{PREFACE TO THE FIRST EDITION}

AFTER I had written special text-books on Pharmacology, including pharmacognosy and pharmaceutical chemistry, on Prescription Writing, and on Toxicology, there remained as a final task, in my restricted department of instruction, General Therapeutics. The present book is therefore a supplemental and concluding volume to the first three.

The presentation of the fundamentals of general therapeutics will always remain a difficult undertaking. No department of medicine undergoes such frequent changes in methods and opinions as therapeutics. A permanent system of general therapeutics cannot be set forth, especially in our own day, in which great changes have occurred in the domain of general pathology, the former absolute domination of the cellular pathology being to some extent shaken by the developments in serum therapy and the old humoral pathology again appearing in the scheme. On the contrary, the discussion must rather be limited to a presentation and interpretation of the present status of the knowledge concerning the subject. This applies especially to the two modern questions of the day in general therapeutics,-namely, disinfection and vaccination. But there are several other questions which at this time have not been definitely settled; for instance, the nature of the antipyretic, diuretic, expectorant, cholagogue, and derivative actions. To find the correct middle ground in the midst of all this uncertainty is not easy. If there is in anything an imminent temptation to present an extreme optimistic or pessimistic conception, it is certainly the case with general therapeutics. At any rate, it is always commendable in a text-book prepared for students and young veterinarians to accept the positive rather than the negative stand-point in doubtful cases. To fill a studious young man at the outset with nihilistic views concerning the efficacy of this or that therapeutic method appears to me to be more hazardous than if one in good faith presented some particular curative system perhaps in a somewhat too optimistic light. There will be an opportunity in practice later to test everything and to retain the best. But if the practitioner, on account of preconceived opinions brought with him from school, excludes one and another curative 
method from this test, there will necessarily result a narrowness in therapeutic methods.

A second difficulty in the preparation of a text-book on general therapeutics is the abundance of material. One must here choose between a broad, voluminous discussion or a short, concise presentation. I am fully aware of the advantages and disadvantages of the two plans. I decided that this book should be as concise as possible, because, for one reason, among others, we already have in our veterinary literature a large and valuable text-book on therapeuties (Ellenberger), in which the assistance of nature in healing and the history of therapeutics especially are given a large amount of space. Therefore, I have limited my discussion of the subject to a presentation of artificial assistance.

There are yet to be subjoined some observations concerning the relations between veterinary curative methods and those of human medicine. There can be no doubt that in veterinary medicine we have derived very many, if not the most, of our therapeutic conceptions from human medicine. But it may not also be known that there are several curative methods peculiar to veterinary medicine, as, for instance, blisters, the actual cautery, lactagogues and ruminatorics; and that some methods, as vaccination and disinfection, are much more extensively employed in veterinary than in human medicine. The prophylactic measures also show, as is well known, a greater stage of development, especially in reference to combating epidemics (sanitary laws), in the domain of veterinary medicine than in that of human medicine, in which in our day preparations were first made for the formulation of sanitary laws. On the other hand, different highly developed curative methods in human medicine can never obtain practical consideration in veterinary medicine,--for example, pneumotherapy, balneotherapy, climatotherapy, mechanotherapy, orthopædics, suggestive therapy, etc. Those who are interested in these subjects must be referred to the text-books on General Therapeutics by Ziemssen, A. Hoffman, and others.

Finally, in justification of the introduction of the numerous etymological footnotes into the book, I may remark that on account of the strangeness of many of the therapeutic terms a short explanation seemed to me to be necessary in the interest of students.

BERLIN.

E. Fröhner. 


\section{CONTENTS}

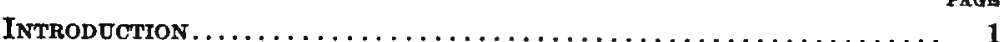

The History of Therapedtics...................... 9

1. Hippocrates........................... 10

2. Galen............................. 12

3. Paracelsus............................ 14

4. Boerharve........................... 17

5. Brown ............................. 18

6. Rademacher........................... 19

7. The Homcopathy of Hahnemann................ 19

8. The Cellular Pathology of Virchow.............. 21

9. The Serum Therapy of von Behring............. 22

10. The Chemotherapy of Ehrlich ................ 23

General Therapeutrcs of the Digeaseg of the Organg of Digestion 25

I. General Therapeutics of tee Diseases of the Stomach 25

1. Dietetic Treatment........................ 29

2. Medicines. (Stomach Remedies. Stomachics)........ 29

3. Mechanical Treatment..................... 33

4. Operative Treatment................... 34

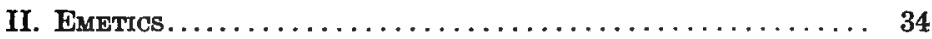

III. General Therapeutics of the Diseases of the Intestines 38

1. Dietetic Treatment..................... 40

2. Cathartics. Laxatives.................... 40

3. Constipating Remedies. Styptics............. 48

4. Mechanical and Operative Treatment........... 51

IV. General Therapedtics of the Diseases of the Liver... 52

1. Cholagogues. Stimulants to Bile Secretion......... 54

2. Dietetic and Mechanical Methods............ 55

General Therapedtics of the Diseases of the Organg of Circu-

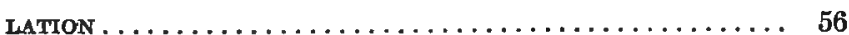

I. General Therapeutics of the Diseases of the Heart... 56

Cardiacs. Heart Remedies................... 57 
II. General Therapeutics of the Diseaseg of the Blood... 60

Dietetic Method. Blood-forming Remedies. Blood Plastics. 63

III. Genfral Therapedtica of the Diseases of the BloodvESSELS............................. 64

1. Methods of Arresting Hemorrhage............ 66

2. Vasomotor Stimulants. Drugs that Contract Bloodvessels............................ 71

3. Vasodilators. Agents that Dilate Blood-vessels....... 72

IV. General Therapeutics of the Exudates and Trangudates.

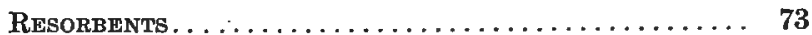

Resorbing Medicines. Resorbents ............... 76

General Therapeutics of Fever................... 80

Medical Antipyretics.......................... 84

Generat Therapeutics of the Diseases of the Nervous Ststem.. 88

Drugs Acting on the Nerves. Neurotics. Nervines....... 90

1. Stimulants to the Nervous System. Excitants....... 93

2. Drugg that Calm the Nerves. Sedatives .......... 97

3. Narcosis and Anæsthesia........................... 101

General Therapedtics of the Diseases of the Respiratort appa-

RATUS ........................... 103

Expectorants............................ 107

General Therapedtics of the Diseases of the Urinary Organs. 112

Diuretics ............................... 113

General Therapeutics of the Diseaseg of the Genital Organs. 118

1. Ecbolics. Abortives....................... 119

2. Drugs that Stimulate the Sexual Impulse. Aphrodisiacs. . . 120

3. Drugs that Depress the Sexual Impulse. Anaphrodisiacs.... 121

4. Drugs that Increase Milk Secretion. Galactagogues.... . . 122

5. Drugs that Depress Milk Secretion. Antigalactagogues... . 127

General Therapeutics of the Glands (Diaphoretics and Siala-

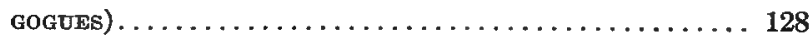

1. Drugs that Stimulate the Secretion of Sweat. Diaphoretics. 129

2. Drugs that Stimulate the Secretion of Saliva. Sialagogues. . 131

Greneral Therapedtich of the Diseages of Metabolism........ 133

1. Plastic Remedies. Plastics................... 134

2. Nutrients. Roborants..................... 137

3. Reducing Remedies. Antiplastics.............. 138 
General Therapedtics of Dismases of the Ere............ 141

1. Pupil-dilating Remedies. Mydriatics.............. 141

2. Pupil-contracting Remedies. Myotics............. 142

Gengral Therapedtics of the Skin and Mucods Membranes.... 143

1. General Therapeutics of the Diseases of the Skin......... 144

2. Cutaneous Irritants. Acrics................... 146

3. Caustics............................... 152

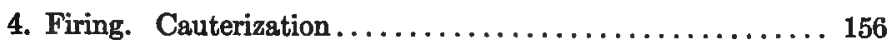

5. Astringents........................ 157

Drdgs that Kill Parasites. Antiparasitics............... 160

1. External Antiparasitics. Antepizoa .............. 160

2. Worm Remedies. Anthelmintics............... 165

Disinfectants. Antiseptics . . . . . . . . . . . . . . . 169

1. Disinfection in General . . . . . . . . . . . . . . . 169

2. Disinfection for Infectious Animal Diseases........... 183

I. Supplement $A$ to the Instructions of the Federal Council, December 25, 1911 (Disinfection in Infectious Diseases)....................... 184

II. The Most Important Disinfectants for Animal Infections 201

3. Disinfection of Wounds...................... 209

4. Internal Antiseptics..................... 213

5. The Conservation of Animal Products............ 214

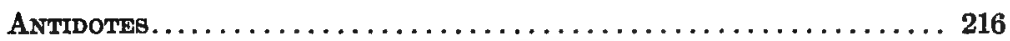

VACCination. ImMUNization. InOcULATION ............. 225

1. Immunity, Mitigation, and Methods of Inoculation....... 225

2. The Different Varieties of Vaccination............ 235

I. Protective Vaccination................ 236

Veterinary Police Regulations Concerning Vaccina-

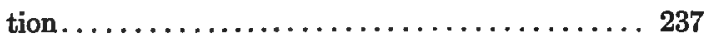

Protective Vaccination for the Different Animal

Infections................... 237

'II. Curative Vaccination................. 252

III. Diagnostic Inoculation. . . . . . . . . . . . 254

WATER as a REMEdT. HYDROTHERAPY.................. 265

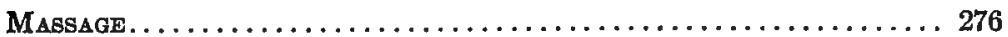

i Electricity as a Remedy. Electrotherapy.............. 282 
BugEDing............................................. 286

General Theraptutice of the Organs of Locomotion (Mugclde, Tendong, Nertegs, Articulations, Bones)................ 288

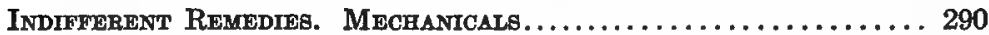

1. Protectives............................... 290

2. Emollients.............................. 291

3. Cleansing Remedies.......................... 292

AIr $\Delta A$ A Remedr..................................... 295

$I_{\text {NDEX }} \ldots \ldots \ldots \ldots \ldots \ldots \ldots \ldots \ldots \ldots \ldots \ldots \ldots \ldots \ldots \ldots, 297$ 


\section{TEXT-BOOK OF \\ GENERAL THERAPEUTICS}

\section{FOR VETERINARIANS}

\section{INTRODUCTION}

Natural and Artificial Healing.-In the healing of disease two possibilities are to be considered. The disease is healed by the curative forces of nature without the assistance of medicines (natural healing), or artificial-i.e., medical-healing processes are necessary (artificial healing). Whether a disease can be left to nature's powers or whether medical treatment is expedient or necessary is the question which the therapeutist must first consider carefully in every case. What may be healed by nature? What may be healed by medical treatment? To these questions may be added a third: What is usually incurable?

1. What May be Healed by Nature?-The answer to this question may be based upon clinical observations and experimental investigations in general surgery and pathology. In surgery as well as in pathology it has been demonstrated repeatedly that the natural healing force and the regenerative capacity of the individual tissue cells and organs are extraordinarily great. The spontaneous healing of wounds and fractures of bones, the reactive protective processes of the body in inflammation (local leucocytosis) and in fever (general leucocytosis), the spontaneous checking of hemorrhage, the organization of a thrombus, the encapsulation of purulent areas and of foreign bodies, the restoration of epithelium and of nerve-fibres, the formation of anastomoses, ankyloses (spavin, ring bone), and nearthroses, the throwing off of gangrenous parts, the resorption processes in pneumonia and pleurisy, the compensatory processes of the heart, kidneys, liver, and lungs, the spontaneous healing and natural immunity of the infectious 
diseases (aphthous fever, strangles, influenza, contagious pneumonia), the spontaneous recoveries from colic in numerous cases, and many other natural healing and corrective processes verify this statement.

A clear insight into the nature of spontaneous healing is afforded by a study of the processes of inflammation. While the inflammatory changes were formerly regarded as harmful and were combated with remedies that reduce inflammation, especially cold, it is now known that inflammation is a healing reaction and should not be opposed but favored. Just as fever, pain, cough, vomiting, diarrhœa, and other disease phenomena exert a natural healing tendency in removing and combating the cause of disease, so do the processes attending inflammation constitute a natural eliminative and healing process. The dilation and hyperæmia of the vessels; the change in the capillary walls by which they become permeable; the out-wandering of the white blood-cells (local leucocytosis) and the exudation of blood-plasma into the tissues; the removal of endotoxins from the inflamed area by phagocytosis; the proliferation of the autochthonous connective-tissue cells; the liquefaction and resolution of the solid inflammatory products by enzymes (lysins); the occurrence of toxin-binding, neutralizing antitoxins, of bacteria-destroying humoral and leucocytic bactericides (bacteriolysins), of bacteria-clumping agglutinins, and of opsonins which prepare the bacteria to be taken up by phagocytosis; the leucocytosis of the blood (lymphocytes, neutrophiles, eosinophile leucocytes); positive and negative chemotaxis; the regeneration of the injured tissue-cells by increased growth of cells; proliferation of tissues and capsule formation-all these are nothing more than protective and healing processes directed against the causes of disease, especially bacteria, and to the regeneration of the injured tissue, such regeneration occurring in living, healthy animal bodies through the adaptability and reproductive powers of the cells. These natural eliminative and regenerative processes should not be disturbed by improper treatment, but should be regarded as natural healing processes and not only favored but even stimulated under certain circumstances. 
2. What May be Healed Artificially?-The problem of artificial assistance consists above all in supporting, promoting, and accelerating the natural healing processes. Natura sanat medicus curat. Disinfected, ligatured, and bandaged wounds, reduced bone fractures, and incised abscesses heal better and more rapidly than when left entirely to nature's efforts. This is also true of the artificial removal of fluid exudates (hydrothorax, ascites, acute hydrocephalus, laminitis), and of the medicinal treatment of heart weakness and dangerously high fever, pulmonary congestion, cerebral hyperæmia (phlebotomy), and numerous other diseases.

In many cases natural healing entirely fails to remove the disease. Then it is only curable through artificial methods. Surgery and obstetrics are especially rich in examples of this kind. To this class of cases belong most of the neoplasms and parasitic diseases, especially mange, deviations in the position of the fotus and of the gravid uterus, invaginations and incarcerations of the intestines, herniæ and prolapses, ulcers and fistulæ, urinary calculi, foreign bodies in the stomach and intestines, tympanites of the rumen and intestines, wounds of the carotid artery, etc.

3. What is Incurable?-The answer to this question willdepend upon the present extent of medical knowledge. There is a large number of diseased conditions which are incurable in spite of all discoveries and therapeutic progress, and which will probably remain so in the future. A dead tissue or organ cannot be replaced in its original form. Incurable also are atrophic conditions, chronic hydrocephalus, chronic interstitial nephritis and hepatitis, ankyloses, many neoplasms in internal organs, fractures of the cervical, dorsal, and lumbar vertebræ, atrophy of the optic nerve, detachment of the retina, emphysema of the lungs, progressive atrophy of the posterior crico-arytenoid muscle (roaring), generalized carcinomatosis, sarcomatosis, tuberculosis, and actinomycosis, echinococcus disease, glanders, and rabies. In the large domestic animals (horses, cattle) fractures of the upper bones of the extremities, severe pelvic fractures, purulent inflammation of the large joints, and perforating wounds of the intestines are, as a rule, incurable. Many diseased conditions are curable in man that are 
incurable in animals, because in the treatment of animals the external conditions necessary to healing are not obtainable in the same degree as in the treatment of man. For instance, many bone fractures in horses and cattle are incurable because a fixation dressing cannot be applied to the part affected. Some operations can not be performed on large animals because of the difficulty of maintaining asepsis. Furthermore, the task of the veterinarian is essentially different from that of the physician. Frequently, the purpose of veterinary therapeutics is not the healing of disease, but to make the animal serviceable. A horse with an amputated leg is, indeed, healed, but is not serviceable. Neurectomy, on the other hand, does not heal ring bone or spavin, but it makes the horse again serviceable. Even economical considerations (value of the animal, time required for treatment) often govern the veterinary therapeutist.

Remedies and Medicines.-The remedies which may be used in the treatment of disease are numerous and various. The term remedy does not signify the same thing as medicine or drug; the latter is only a special form of the former. There is not always, as is frequently incorrectly assumed by the laity, a sharp contrast between medical and drugless treatment (so-called natural method). On the contrary, the same therapeutic effect produced by drugs may frequently be obtained from other, non-medical remedies,e.g., through mechanical methods. In general, dietetic, chemical (medicines), mechanical (massage, hydrotherapy), thermic (heat, cold), electrical (electrotherapy), and operative measures are to be considered as therapeutic remedies. Also of importance as healing factors are rest, movement, and exercise. Disinfection, inoculation, and sanitary police regulations are of especial value in combating infectious diseases.

If, after the use of this or that therapeutic measure, the disease is cured, this is not in itself proof that the treatment applied was the cause of the cure. Post hoc is not propter hoc. On the contrary, every recovery should be considered candidly and objectively to determine what share in the cure is to be credited to the healing powers of nature and what part was played by the therapeutic 
measures employed. The observance of this rule is very necessary, especially in veterinary therapeutics. Fallacious, speculative conclusions from meagre casuistic material, and unjustifiable commendation and overrating of new remedies are in veterinary medicine almost more common than in human medicine.

Healing Methods.-General therapeutics differs considerably from special therapeutics and pharmacology. The latter considers in a detailed manner the actions and uses of the individual medicines in the different diseases. General therapeutics embraces the different views concerning the treatment of diseases and the action of remedies in general. Out of the sum of individual observations it constructs certain general rules and laws, on which are based the employment and systematic grouping of the curative agents. Such a consideration of the healing agents naturally leads to the formulation of the so-called healing methods. General therapeutics can therefore be defined as the study of the healing methods.

Thenumber of healing methods has been large from the remotest times. From an entirely general standpoint there are usually distinguished as special healing methods the direct, indirect, and derivative, the local and general, the causal, radical, and symptomatic, the empirical, statistical, rational, and physiological, and the prophylactic, expective, abortive, and vital. Concerning the nature of these methods, the following may be stated:

1. The direct healing method consists in the direct or immediate application of the remedy to the disease (indicatio morbi). A direct therapeutic process, for example, is the employment of antiseptics, antiparasitics, and antidotes-the bacteria, parasites, and poisons concerned being directly influenced. Other examples are the use of caustics and many operations (extirpation of tumors, removal of foreign bodies).

2. The indirect healing method attacks the disease through the medium of the circulation and the nervous system. It is, therefore, also called the general method. This method includes the dietetic medicines, which influence disease through nutrition and metabolism; the resorbents and derivatives, which act through the 
circulation; and cutaneous irritation, which operates reflexly through the nervous system upon the distant disease process. The derivative action is also regarded as an independent healing method. This action can be attained through the operation of cutaneous stimulation, cathartics, diuretics, diaphoretics, sialagogues, and bleeding. By far the greater number of cases of all diseases are treated by the indirect method, because only in isolated cases can the therapeutic measures be applied directly to the disease.

3. The causal method attacks not the disease itself but the cause, e.g., in the infectious diseases, the bacteria; in poisonings, the poison. To this extent it agrees with the direct method. With reference to its value, it must be observed that the removal of the cause is only possible in certain diseases (surgical measures, antisepsis, antiparasitics, antidotes, emetics, cathartics). Furthermore, after the removal of the cause the disease frequently does not cease, e.g., rheumatic diseases after the removal of the cold irritant; and finally, in many diseases the cause is either not accessible (distomatosis, echinococci) or is not known (ultravisible virus, epilepsy, diabetes mellitus).

4. The radical method is directed against the root or source of the disease and not merely to the removal of the associated disturbances. As the definition indicates, the radical method is related partly to the causal and partly to the direct (local) method. Goitre and actinomycosis may be treated indirectly through the medium of the blood stream with iodine. The radical method consists in the operative removal of the diseased parts. In a similar manner are herniæ and urinary calculi treated radically,-i.e., by operation. Unfortunately, there are only a small number of diseases in which complete restoration can be attained by the radical method.

5. The symptomatic or palliative method is directed against neither the cause of the disease nor the disease itself, but only against the symptoms of the disease. In the infectious diseases, for example, the fever is treated, in laryngeal catarrh the cough, in gastric catarrh the vomiting, in intestinal catarrh the diarrhoea, in brain diseases the psychic excitement and convulsions, in lameness 
the pain (neurotomy). Practical experience teaches that in very many cases treatment must be confined to this method.

6. The rational or physiological method is based upon scientific observations and experimental investigations of the cause, nature, and pathogenesis of the different diseases, and the action of the individual medicines. The therapeutist seeks to explain scientifically the action of his remedies upon the basis of the contemporaneous knowledge of therapeutics. Inflammation and the infectious diseases, for instance, are caused by the action of microorganisms and are therefore treated with antiseptics; the diseases of the heart with drugs whose physiological action upon the heart has been exactly investigated (digitalis).

7. The empirical healing method is, in contradistinction to the rational, based upon practical experience only, without the nature of the disease and the action of the remedies being considered scientifically in connection with each other. This method includes the former common employment of potassium nitrate, calomel, phlebotomy, and the other so-called antiphlogistics in the inflammatory diseases. It should be the endeavor of scientific therapeutics to extend the rational method more and more and to reduce empiricism. On the other hand, it must not be overlooked that our knowledge of the nature of disease and of the action of healing remedies is not yet so complete that the empirical method can be dispensed with entirely. Furthermore, many valuable therapeutic measures, which are at this time regarded as rational, were discovered in a purely empirical manner (massage, hydrotherapy, folk-medicines). The statistical healing method is in a certain sense a subdivision of the empirical. It is based upon the statistical demonstration of the effectiveness of a remedy or a cure.

8. The expectant or waiting method leaves the mastery of the disease to the healing powers of nature and resorts to medication only when the automatic regulation of the body fails. It conforms in part to the dietetic method, and is very properly practised more in recent times than formerly. Especially in the infectious diseases with a typical course (influenza, contagious pneumonia, strangles, foot-and-mouth disease) is therapeutic interference 
indicated only when certain abnormal complications appear (very high fever, heart weakness, diarrhce, etc.). The same principle also applies to catarrh of the upper air-passages, gastric catarrh, intestinal catarrh, and to numerous surgical conditions (distortions, tendinitis, spavin, etc.).

9. The prophylactic method is, strictly speaking, not a curative method, but a process of preventing the occurrence of disease and its extension to healthy animals. Nevertheless, it is of great importance ("Prevention is better than cure"). It consists in attention to hygiene, dietetics, rational breeding and feeding, disinfection, and the sanitary police measures for controlling disease.

10. The abortive method combats disease in its initial or formative stage. Emetics and calomel appear to have an abortive effect against certain infectious diseases (canine distemper, swine erysipelas) in consequence of their action in removing the cause of the infection from the body. In poisonings an abortive cure can be spoken of in the same sense. This is also true of arecoline and phlebotomy in laminitis and in cerebral inflammation, likewise of amputation of the tail in tetanus resulting from infection of a wound in the tail. On the other hand, the claim that contagious pneumonia of horses is influenced by the intravenous injection of salvarsan, in the sense that inflammation of the lungs does not develop or that complications and secondary diseases do not occur, does not appear to have been proved.

11. The conservative method aims at the greatest possible conservation of the diseased organ. In veterinary surgery it has a certain importance (preservation of cutaneous flaps in abraded wounds and also in wounds of the wings of the nostrils and of the eyelids).

12. The vital method (vital cure, indicatio vitalis) concerns itself with the preservation of life when it is suddenly threatened in the course of a disease. It is really a symptomatic method (tracheotomy in pharyngitis and œdema of the glottis; bleeding in œedema of the lungs; puncture of the pleural cavity, peritoneal cavity, the rumen, intestines, and bladder when collections of fluids or gas threaten life). 


\section{THE HISTORY OF THERAPEUTICS}

THE history of therapeutics, or medicine, extends far back into the remotest antiquity. The earliest traditions are derived from Indian (Upavedas, Agurveda, Susrutah des Dramoantari), Egyptian (Isis, Osiris, Horus, Harpocrates, priest medicine), and Hebraic (Moses, Levites, prophets, Essenes) literature. Very old also is the Chinese medicine (Ching de chung Ching). But the real scientific therapeutics begins only with the Greeks, with an introductory period which may be designated as the philosophical (Thales of Miletus, Pythagoras, Alcmæon, Empedocles, Anaxagoras, Democritus). The Grecian medicine was also in the beginning a "priest medicine." The so-called Asclepiadæ were associations of priests, purported to be founded by Asculapius the god of medicine, who held temple-polyclinics in which they practised their secret medical art, which was transmitted by oral communication. Out of one of these temples of Asclepiadæ at Cos came Hippocrates (400 B.C.), the founder of Grecian medicine. His teachings, the humoral pathology, entirely dominated Grecian and later also Roman medicine; its influence extended even through the middle ages into modern times (sixteenth and seventeenth centuries). The writings of Galen (131-200 A.D.), which were regarded as medical dogma of Biblical authority throughout the entire middle ages, a period of not less than fifteen hundred years, are nothing more than an amalgamation of the medicine of Hippocrates with the philosophy of Plato. The Arabian school (9001000 A.D.) introduced by Rhazes and Avicenna, and the so-called Monks' medicine, especially the school at Salerno (about 1100 A.D.), were also founded upon the teachings of Hippocrates. The Arabian school also included the new factors of alchemy and spiritualism.

In the sixteenth century, Paracelsus (1493-1541) began the actual reformation of the Galenic and Hippocratic teachings. Believing chemistry to be the basis of therapeutics, he created his 
system of chemiatrics or iatrochemistry, but, as he was strongly under the influence of the spiritualism of the Arabian school, his ideas in part acquired a mysticism. The scientific foundation of modern therapeutics was laid in the period from the sixteenth to the eighteenth century by exact anatomical, pathological, physiological, and clinical-pharmacological investigations. Prominent in this work were Vesalius (1514-1564), Fallopius (1523-1562), Malpighi (1628-1694), the founder of modern anatomy and histology ; Morgagni (1682-1771), the creator of pathological anatomy; Harvey (1578-1657), the discoverer of the circulation of the blood and founder of physiology; Ambroise Paré(1517-1590), the reformer of surgery, and Sydenham (1624-1689), the master of practical clinical medicine.

A special position in the modern history of therapeutics is held by Boerhaave (1668-1738), the founder of the modern humoral pathology (hæmatopathology); Brown (1735-1788), the author of Brownianism; Rademacher (1772-1849), the author of the experience or empirical method; Hahnemann (1755-1843), the father of homceopathy, and also several discoverers of natural healing methods, especially Hufeland (1762-1836), Schönlein (1794-1864), and Priessnitz (1799-1851).

The history of modern therapeutics in the nineteenth and twentieth centuries lacks the prominent, central character of former periods, because it consists of the special history of the several branches into which medicine has been divided. Of general curative methods, only three are of importance: the cellular therapy of Virchow, the serum therapy of von Behring, and the chemotherapy of Ehrlich. The first locates the disease and also the healing powers in the cells, the second uses the therapeutic action of the immune bodies of the blood-serum (antitoxins), and the third treats certain infectious diseases (protozoa, trypanosomes, spirilla) with specific chemical substances.

\section{HIPPOCRATES}

Biographical.-Hippocrates was born in the year 460 B.c., on the island of Cos, in Asia Minor, the son of Heraclides, one of the 
Asclepiadæ and a teacher in the medical templè school at Cos. He was a contemporary of Pericles. He travelled in Asia Minor, Greece, Scythia, and Lybia and resided subsequently in Thessaly, where he died in Larissa in 364 (375?). He published the medical secrets of the priests of the Asclepiadæ and his own experiences in several books (Aphorisms, Prognostics, Epidemics, Treatment of Inflammatory Diseases, Wounds of the Head, Herniæ). His expression, "Life is short, art is long, opportunity fleeting, experience deceptive, judgment difficult," is well known.

The Theory of Hippocrates.-The humoral pathology of Hippocrates attributed all diseases to changes in the fluids of the body. The body contained four cardinal fluids or humors: the blood, the mucus, the yellow and the black bile. The normal mixture of these four fluids (i.e., health) is the crasia, while an unequal mixture generates disease, or dyscrasia. The problem of therapeutics is to change dyscrasia into crasia. This can be accomplished in three ways: 1 , by removal of the superfluous fluid,-e.g., of the blood by phlebotomy, of the bile by cholagogues, of mucus by drugs that increase the secretion of mucus (derivative, depletive method); 2 , by altering or rendering harmless the superfluous fluid in the body by cooking, ripening, or transforming,--pepsis, coctio, maturatio, alteratio (alterative method); 3 , by restoring deficient cardinal fluids (dietetic method).

In addition to the crasia theory, Hippocrates also formulated a crisis theory. According to this latter theory the fever reaches the crisis or turning point on certain so-called critical days. The seventh day especially was regarded as the critical day and as the proper time for therapeutic interference. Depleting drugs especially were administered on these days to increase the critical eliminations. Cathartics and emetics, especially the vegetable drastics (veratrum, euphorbium, daphne), were used for their derivative action. Phlebotomy was employed to reduce fever only in strong and full-blooded individuals. In addition to the external remedies, he assumed the presence of an internal, primitive healing force which induces the crisis. 
Aristotle, also of a family of Asclepiadæ; lived from 384 to 322 в.с.; was teacher of Alexander the Great; pupil of Plato; founder of natural history and comparative anatomy; teacher of the elementary qualities; discovered the nerves and gave the aorta its name.

Herophilus and Erasistratus, celebrated anatomists of the Alexandrine school (time of the Ptolemies). Herophilus discovered the sensibility of the nerves, the finer anatomy of the eye, distinguished between systole and diastole, and named the duodenum. Erasistratus discovered the lymphvessels and healed liver abscesses by operative incision.

\section{GALEN}

Biographical.-Claudius Galenus was born in Pergamos, 131 A.D., during the reign of the Emperor Hadrian. He was the son of a builder. After pursuing a course of study in philosophy, he studied four years in the school of the Pergamonic physicians. He then visited Smyrna and Corinth, Asia Minor and Palestine, and finally the at that time celebrated medical school at Alexandria, where anatomy especially had flourished from ancient times and where alone the dissection of the human body was permitted. In addition, toxicology was there thoroughly taught. Poisons and antidotes at that time formed the chief part of pharmacology. Returning to Pergamos in 159, he was appointed physician to the gladiators. In 165 he received an appointment under the Emperor Marcus Aurelius in Rome, where he gave lectures on physiology and prepared the royal electuary, a mixture of 62 drugs. The prescription was written by Andromachos, the physician of Emperor Nero, and was obtained from the Alexandria school. In 180 Galen was the physician of Emperor Commodus, and in 193 of Emperor Septimius Severus. He died in the year 200 A.D. His numerous writings, in all about 500, were destroyed in great part through the burning of the Temple of Peace in the reign of Commodus. Eightythree medical works were preserved, among them the writings on "Healing Methods," "Critical Days," "Functions of the Parts of the Human Body," "Combination and Force of the Simple Medicines," and "Differentiation of the Different Varieties of Pulse."

The teachings of Galen were later acknowledged even by the church; doubt of their correctness was regarded as sacrilege. His 
dogmas received a consideration only equalled by that given to the Bible, and because of this were a serious hindrance to the development of medicine for the succeeding fifteen hundred years. When the students of medicine explained that dissection was necessary to discover the errors of Galen, the church forbade the opening of human bodies and stated that Galen never could have erred and that dissections were therefore not only unnecessary but would be reprehensible.

Pergamos, his native city, had golden medals struck in honor of Galen, and he was for other reasons well aware of his position, as is shown by the following statement: "Hippocrates indeed had made something of a track and broken the path, but I have smoothed it and made it passable, as Emperor Trajan did with the military roads in the Roman Empire."

The Galenic Theory.-The humoral pathology of Hippocrates, with its four cardinal fluids and the crasis and crisis theories, was the nucleus of the Galenic system of medicine. In addition to dyscrasia, Galen regarded as causes of disease changes in the so-called elementary qualities (heat, cold, dryness, and moisture). Natural, primary forces of the body were attraction, adhesion, secretion (apocritical), and elimination. He assumed that every medicine possessed specific elementary qualities. In his anatomical and physiological studies he came very close to the discovery of the circulation of the blood. He was convinced that respiration served to maintain the body heat; he compared the respiration with combustion and contended that the flame and animal life were supported by the same constituents of the atmosphere. These constituents he called "air spirits," and, after they were taken into the blood, "life spirits" (spiritus vitalis). Fever was an unnatural change in the temperature. The lightest form of fever, the "ephemeral," occurred when only the "air spirits" were embarrassed. If the blood and fluids were affected, there arose "septic" or putrid fever. When the heart and solid parts of the body became hot, then the fever was "hectical." A fever continuing one day was due to mucus; three days, to yellow bile; four days, to black bile. 
Arabian Medicine.-Its foundation was the theories of Hippocrates and Galen. Added to these were the new elements of chemistry and pharmacy. As alchemists, the Arabian physicians discovered analysis, synthesis, distillation, sublimation, precipitation, preparation of salts, and the manufacture of alcohol. They were also believers in spiritualism, and considered medicines as the bearers of a spirit, to which their power was due. The most celebrated Arabian physicians were Rhazes (923 A.D.) and Avicenna (978 A.D.). Rhazes was first a teacher of medicine and philosophy in Bagdad, later director of the lazaretto in Ray. His works were: "On the Healing of Diseases," "Aphorisms," and "Antidotes." They contain the earliest discussion of smallpox. Medicines mentioned are: mercurial preparations, copper sulphate, arsenic, nitrate of potash. Avicenna, a native of Bokhara, studied in Bagdad, was private physician to the prince of Ray and later Vizier in Hamadan. On account of an insurrection be fled to Ispahan, dressed as a monk. To his chief works he gave the name of "Canons"; they contained nearly everything concerning the entire subject of medicine and attained a wide circulation in Europe. His catalogue of medicines was very extensive (rhubarb, silver, gold, many plants).

Monks' Medicine.-From the sixth to the sixteenth century, a period of one thousand years, in Italy, Germany, France, and other countries of western Europe, medicine was almost exclusively in the hands of the monks, who conducted special medical schools. Of the latter the most celebrated was the school at Salerno, from the tenth to the thirteenth century, a Neapolitan Benedictine cloister, where pharmacy, pharmacology, and dietetics especially were taught (Regimen sanitatis Salerni, Antidotariam minus, De simplici medicini, Eros). Another Neapolitan cloister school was the one at Monte Casino. In France, in the thirteenth century, was the school of Montpellier, which later developed into the University.

\section{PARACELSUS}

Biographical.-Philippus Aureolus Theophrastus Bombastus ab Hohenheim, called Paracelsus, was born near Zurich in 1493, the son of a physician. He studied in Basel and travelled in almost all the countries of Europe. In 1527 he became professor of physics, medicine, and surgery in Basel and also city physician, but left there secretly a year later on account of differences with the municipal authorities. After that he lived an unsettled, wandering life in a number of cities in Alsace, Bavaria, Würtemburg, Austria, Switzerland, and other lands, until, at the age of 48 years, he died in 1541, in Salzburg, where he was buried. Besides a large number 
of philosophical and theosophical works he wrote numerous medical books. Of the latter the most important are: "Commentary on the Aphorisms of Hippocrates," the "Three Great Books on the Treatment of Wounds," "The Hospital Book," the "Seven Books on Open Wounds," "Lectures on Wounds," "Minor Surgery," "The Bath of the Priests," "Booklet on the Pestilence in the City of Stertzingen," "On the Gout," "On Syphilis." Opinion concerning the influence of Paracelsus as a reformer of medicine was formerly very much divided. Disregarding his personal shortcomings, with which his pupils especially reproached him, and his inclination toward mysticism, there can be no doubt that Paracelsus, by his thorough refutation of the crasia theory and humoral pathology of Hippocrates and Galen, and through his own new, chemiatric system, exerted a very great effect upon the development of medicine.

The Theory of Paracelsus.-The so-called chemiatric, chemical or spagyrical system of Paracelsus was the first to associate the chemical properties of medicines with their action upon the body. He called these properties "virtue and force in medicines." In opposition to the humors and elementary substances of Galen, he attributed life and disease to organic processes within the body, and spoke of a "vita propria" of the organs. Chemistry, in his opinion, was the foundation of all therapeutics. We owe to him the introduction into the materia medica of several important chemicals, especially iron and its compounds, the preparations of antimony, sulphur, copper, zinc, and sodium nitrate. He also gave exact indications for the use of the mercurial preparations. His chief remedy was opium in the form of a tincture, called after him Tinctura Paracelsi. In place of the formerly exclusively used decoctions, he used tinctures, extracts, and essences. He also called attention to numerous chemical combinations that were incompatible, and was the first to discuss the composition and action of mineral waters. His efforts in the domain of surgery were also considerable. He was the first to declare that the surgeon should also be a physician. Pus, he said, was a "natural balsam" which favored cicatrization, and its healing action should not be interfered 
with by the excessive use of salves or plasters. In his philosophical writings two terms frequently occur which are used in modern natural science: macrocosmus and microcosmus. The first term, as he used it, indicated nature as a whole, the latter the separate individuals.

Vesal.-Andreas Vesal (Vesalius), founder of modern anatomy, was born in Brussels in 1514, but had to flee from Belgium on account of his activity in anatomical work. He studied in Paris under Sylvius, held in Italy the position of demonstrator of anatomy, and in 1537 was called by the Republic of Venice to be professor of anatomy at Padua, after he had published his celebrated work "De corporis humani Fabrica libri septem" in 1535. Subsequently he was private physician to Karl V and Philip II in Madrid. Condemned to death by the Spanish inquisition as a magician or conjurer, he was pardoned by Philip II to take a penitential journey, and died in 1564 on a pilgrimage to Jerusalem. Vesal opposed the theory of Galen, and demonstrated, among other things, that Galen's anatomy was founded upon the ape and not upon man.

Fallopius.-Gabriel Fallopius, pupil and successor of Vessl, was born in Modena in 1523, and from 1547 on was professor of anatomy and surgery in Ferrara, Pisa, and Padua. He wrote "Observationes Anatomicæ" in 1561 and discovered the Fallopian canals in the temporal bone.

Malpighi--Marcellus Malpighi, 1628-1694, discovered the capillary circulation and laid the foundations of the microscopical anatomy of animals and plants (Malpighian corpuscles of the kidneys). He was professor of anatomy in Bologna, Pisa, and Messina, and, at the time of his death, private physician to Pope Innocent XII.

Morgagni.-Giambattista Morgagni, founder of pathological anatomy, pupil of Valsalva, born in Forli in 1682, was called in 1711 to the University at Padua to the chair formerly held by Vesal. Here he attracted numerous students from all lands, especially Germany. In 1761 he issued, in five books, his famous work: "De Sedibus et Causis Morborum." His name is a part of several anatomical terms (Morgagnian liquor between the lens and its capsule, Morgagnian cavity). He died in 1771, 89 years old.

Harvey.-William Harvey, founder of physiology, pupil of Fabricius ab Aquapendente, born in Folkstone in 1578, elected professor of anatomy in London in 1615, discovered the circulation of the blood in 1628. He was the author of the conclusion "omne animal ex ovo," and showed that, contrary to Galen, the blood was not one of four fluids, but the only vital fluid ("humor cardinalis").

Ambroise Paré.-Ambroise Pare, founder of modern surgery and obstet- 
rics, lived in Paris from 1517 to 1590 , and became known through his use of ligatures on the blood-vessels in operations.

Sydenham.-Thomas Sydenham, 1624 to 1689 , physician in London, reformed practical clinical medicine, bringing it back to the methods of nature and of experience. Symptoms of diseases were regarded by him as the effort of nature to eliminate the disease materials. The tincture of opium was named after him Tinctura Sydenhami.

\section{BOERHAAVE}

Biographical.-Herman Boerhaave was born in the Netherlands in 1668 , the son of a clergyman. He becameprofessor of medicine, botany, and chemistry in Leyden, where he published his celebrated works: "Institutiones medicæ in usum annuæ exercitionis" (1708) and "Aphorismi de cognoscendis et curandis morbus" (1709). In addition to these, he published the "Elementa chemiæ" (1718). He died in 1738. His best-known pupil was van Swieten (1700-1722), the private physician of the Empress Maria Theresa and the founder of the Vienna school.

The System of Boerhaave.-Boerhaave can be regarded, to a certain extent, as the founder of the modern humoral pathology, since he pointed to the chemical changes in the composition of the blood as the cause and essence of disease. His humoral pathology is therefore a hæmato-pathology. The treatment recommended by him was the removal from the blood of the injurious substances or irritants by means of cathartics, diuretics, disinfectants and resolvents. He assumed the presence of different kinds of harmful irritants in the blood: acid, alkaline, salty, fatty and glutinous. In his opinion organic life consisted of motion. Fever he regarded as the effort of life to overcome death (compare the concurrence of this view with the modern idea that fever is a protective reaction against the infectious material). Worthy of note also is his conception of inflammation, which he regarded as a complete stasis of blood in the smaller vessels.

F. Hoffmann.-Founder of the so-called iatromechanical or mechanicodynamic school; born in Halle 1660; professor in Halle 1694; private physician to King Frederic I and professor in Berlin 1708 to 1712; died in Halle in 1742. His name is preserved in the preparation introduced by him known as "Hoffmann's drops." 
Stahl.-Founder of the chemical phlogiston theory and the spiritual theory (principal work: Theoria medica vera, 1701); expective method of treatment. Born in Ansbach in 1660, professor in Halle 1694; private physician to King Frederic William I in Berlin 1716; died 1734. Stahl's ointment for burns and Stahl's pills perpetuate his name.

Albrecht von Haller.-Physician, anatomist, physiologist, botanist, and poet. Discoverer of the irritability of the muscle fibres. Born in Bern 1708; professor in Göttingen 1736; president of the Royal Society of the Sciences. Died in Bern 1777.

\section{BROWN}

Biographical.-F. Brown was born in Bumbe,England, in 1735. At first a linen weaver, he later studied theology and medicine and was a physician in London. His principal work, "Elementa medicinæ," was written in 1780 . He died in London in 1788. His most celebrated follower was the Italian, Rasori (died 1837).

Brown's Theory.-Brown was the founder of the so-called Brownianism, which was in direct opposition to the humoral pathology and attributed all disease to a deficiency or excess of stimuli or excitability. A medium degree of stimulation or excitability constituted health. Disease was due to either an increase of the excitability (sthenia) or a decrease (asthenia). Medicines also were sthenic, i.e., strengthening (alcohol, camphor, arnica), or asthenic, i.e., weakening (bleeding, hunger). The one-sided Brownian theory was later transformed by Röschlaub into the socalled "stimulation theory" and by Rasori into the theory of "contra-stimulus" (irritant and counter-irritant). These two modifications of Brownianism are also only of historical interest.

Hufeland.-An opponent of Brownianism, he contrasted the vital forces, vis vitalis, with the natural healing forces, vis nature medicatrix, and promoted especially the antipyretic method of treatment (cold-water applications). His chief work was entitled "Makrobiotik," published in 1796. Born in Langensalza in 1762, he died in 1836 in Berlin, where he was a professor and private physician to King Frederic William III.

Schönlein.-Founder of the expective method of treatment. He regarded disease as an independent process which must be allowed to run its course. He taught medicine from the stand-point of the natural sciences and introduced methods of clinical examination. The foundations were laid by him of the so-called natural-history school and the later physiocratic or natural 
healing method (in contrast to the technocratic or artificial healing method). He was born in Bamberg in 1793, was a professor and clinician in Würzburg, Zürich, and Berlin from 1839 to 1859 , and died in 1864 .

\section{RADEMACHER}

System of Treatment.-Rademacher (born in 1772 in Hamm, physician in Goch, on the lower Rhine, died 1849) is the founder of the system of treatment based on experience, or the empirical method. In his work, "Rechtfertigung der Erfahrungsheillehre der alten scheidekünstigen Geheimärzte," heassumes, entirely upon empirical grounds, the existence of specific relationsbetween certain medicines and certain individual organs. Diseases he divided into two classes: "organic diseases" and "universal diseases," and he also recognized two corresponding divisions of medicines: "organic remedies" with local action and "universal remedies" with multiple action. He tested the effect of the individual organ remedies and universal remedies upon the different diseases and named the disease after the remedy found to be effective. The local diseases, for example, were named: digitalis disease of the heart, chelidonium disease of the liver, and antimony disease of the lungs, while the universal diseases were called: copper disease, iron disease, saltpeter disease. In this way he caused the diagnosis to be based upon the therapeutics. While the fundamental thoughts of the Rademacher system concerning the importance of experience and the specific local action of drugs may be acceptable, his nomenclature and many of his conclusions certainly are not. His influence upon therapeutics was therefore only temporary.

Natural Healing Method.-The author of the natural healing methodi.e., the drugless treatment of disease with water, diet, heat, exercise, rest, air, and dressing-was Priessnitz (1799-1851). With reference to his method, see chapter on hydrotherapy. In addition, the following are also to be mentioned as followers of the natural healing method: Schroth (dry diet, dry-bread cure), G. Jäger (wool), Lahmann (cotton wool), and others.

\section{THE HOMCEOPATHY OF HAHNEMANN}

Biographical.-Samuel Hahnemann, the founder of homœopathy, was born in Meissen in 1755. He practised medicine in 
Köthen from 1820 to 1835 , when he moved to Paris, where he died in 1843. Hahemann came into prominence with his new doctrine in 1796. His chief works were: "Organon der rationellen Heilkunde" (1810) and "Reine Arzneimittellehre" (1811).

Hahnemann's Theory.-Disease, according to Hahnemann, consists of the symptoms only. The treatment of disease, therefore, consists of combating and removing the symptoms (symptomatic method). Certain drugs produce in the healthy organism symptoms like those caused by disease. Digitalis, for example, produces symptoms exactly similar to those of heart disease. In the treatment of any disease, therefore, a drug should be used that will produce in a healthy organism symptoms similar to those observed in the particular disease; in heart disease, for instance, digitalis. Hence the motto of homcopathy: Similia similibus curantur.

Hahnemann further maintained that every drug acted the more powerfully the more it was diluted. For this reason a drug should always be administered in extreme dilution, i.e., in the smallest dose possible. Moreover, only one drug should be used at a time. Allopathy, according to Hahnemann, acts contrary to the purpose. There are three forms of homœopathic preparations: Triturates, dilutions, and pellets. A triturate of one part of the drug and 99 parts of milk sugar is called the first triturate; one part of the first triturate with 99 parts of milk sugar forms the second triturate, and so on. Similarly, the first dilution or potence is a solution of one part of thedrug in 99 parts of alcohol, the second dilution or potence a solution of one part of the first dilution in 99 parts of alcohol, etc. With these dilutions the pellets, which are made of milk sugar, are moistened.

The charlatanism of the homøopathic theory is not without effect upon thelaity even to-day. However, it is more to be deplored that Hahnemann has found many followers in medical circles. If, on the one hand, the so-called newer homcopathy has sought to coincide more with modern investigation, and if, on the other hand, serum therapy in many respects has assumed a homœopathic char- 
acter, this cannot change the judgment concerning the homoopathic fundamental of potency or dilution, which is far too inconsistent to be accepted by the healthy human understanding.

Isopathy.-A variety of homœopathy, which treated "like with like" (aqualia arqualibus curantur), e.g., the use of roasted proglottides against tape-worms and the administration of powdered pieces of carcinoma in the treatment of carcinoma. The preparations of the modern organotherapy (thyroid gland against goitre, prostate against diseases of that gland, ovaries in ovarian diseases, spermatozoa in impotence) and also vaccination (Jennerization) show a close relation to the old isopathy.

Mesmerism.-Mesmer, the originator of mesmerism or the theory of animal magnetism, was a physician in Vienna and Paris (1734 to 1815). He claimed to be able to cure disease by touch and stroking, through a "magnetic force" that passed from him to the patient. Mesmer was the forerunner, on the one hand, of the fraudulent spiritualism, and, on the other, of hypnotism (suggestion), which in recent times has become an important therapeutic factor for man.

\section{THE CELLULAR PATHOLOGY OF VIRCHOW}

Definition of Cellular Pathology.-According to Virchow, there are no general or universal diseases. On the contrary, the pathologist finds in every diseased body a considerable, indeed, as a rule, by far the greater part of the organs normal. A diseased body, in which every part is changed, is never seen. The diseased or the inert portion includes only a part of the body. The question that confronts the physician is: where is the disease ( $u b i$ est morbus)? and he must be able in every case to point out the location of the malady in the body (principle of localization). The scientific investigation with reference to the situation of the disease (Sedes morbi) is extended to the tissues and finally to the cells of the organs which show the actual areas of disease (cellular pathology).

In some diseases, especially many nervous diseases and intoxications, visible anatomical changes are not present in the organs or cells. Nevertheless, the localization of the disease in such cases must be established upon a physiological or chemical basis, even in the absence of anatomical alterations which can be demonstrated by the usual methods. 
As a basis for treatment, Virchow's theory requires that medicines must exert a local action upon the diseased organ or its cells (cellular therapy, localized therapy).

\section{THE SERUM THERAPY OF VON BEHRING}

Definition of Serum Therapy.-The etiological investigation of the infectious diseases begun by Pasteur and Koch resulted in an etiological therapy, which was first used by Lister in surgery (antiseptic treatment of wounds). This etiological principle was introduced in internal medicine especially by von Behring. His system, because of its specific, pronounced humoral pathological character, is the direct opposite of the cellular pathology theory. According to von Behring, in the course of infectious diseases in the animal or human body chemical bodies are formed, especially in the bloodserum, which can be employed not only as protectives but also as curative agents against these diseases. The so-called antitoxins are specific antidotes to the poisons (toxins) and bacteria of these diseases; some neutralize chemically the poisons formed by the pathogenic bacteria (antitoxic action), others destroy the pathogenic organisms (bacteriolytic action). They form the foundation of the modern serum therapy or isotherapy. Recently, isotherapy has developed in two different directions, which are to be distinguished as isotherapeutic and homœotherapeutic principles in the restricted sense. Isotherapy uses the so-called isobodies for immunization, i.e., the same agent which causes the disease to be combated: the bacteria themselves. It includes Jenner's method of vaccination against smallpox, Pasteur's system of anthrax vaccination, and von Behring's protective vaccination against tuberculosis. Homcotherapy does not use the living or killed organisms, but the toxins or antibodies produced by them, and may therefore be called isotoxic therapy. To this division belong von Behring's diphtheria serum, tuberculin and mallein, tetanus, swine erysipelas and hog cholera serum, etc. For more extensive details of serum therapy see the chapter on immunity and vaccination. 


\section{THE CHEMOTHERAPY OF EHRLICH}

Definition of Chemotherapy.-Formerly, the pharmacology of medicines was tested only on healthy animals, and only drugs which produced symptomatic effects were included in the tests. Medicines which act specifically were tested rarely (quinine against malaria, mercury and iodine against syphilis). The new experimental therapeutics produces certain infectious diseases artificially in experimental animals and studies the action of curative agents upon these diseased animals. Specific medicines are also produced synthetically for use in the treatment of certain infectious diseases.

Ehrlich has shown experimentally that the specific relations of the curative agent to different parts of the body, its so-called tropic properties, may be very different. A distinction must be made between the organotropic action, i.e., the relation to certain organs (neurotropic action, etc.), and the parasitotropic action, which is exerted not upon the animal body itself but upon the parasites present in the body. In serum therapy, products of the body act as protective agents in a purely parasitotropic manner and without any organotropic effect. Since the body and its cells are not influenced by these protective substances, serum therapy excels any other method of treatment in those cases to which it is applicable. But serum therapy cannot be employed in some of the infectious diseases, e.g., in malaria, trypanosomiasis, and spirillosis. In such cases, chemical antiparasitic remedies must be used (chemotherapy instead of serum therapy). These chemical substances, however, are mostly poisons, which are not only harmful to the parasites but also to the organism (parasitotropic and organotropic action). In chemotherapy, only such medicines can be used as will kill the parasites without doing any considerable harm to the body. Corrosive sublimate, carbolic acid, and arsenic are not suitable on account of their strong organotropic action. On the other hand, the extremely poisonous organotropic action of arsenic can be reduced by certain synthetical combinations and the parasitotropic action relatively increased. The first of these synthetic, chemotherapeutic, arsenical preparations were atoxyl (sodium arsanil- 
icum), an amino derivate of phenyl arsin, an organically combined arsenious acid, and arsacetin, an acetyl compound of atoxyl. The knowledge that only the trivalent arsenic group exerts a poisonous effect upon the cells of the body led by degrees to synthesis of arsenophenylglycin and salvarsan, organic arsenical preparations with a predominant, parasitotropic, specific action against syphilis and spirillosis. A remedy with a similar action against trypanosomes is trypanred. The complete and immediate sterilization of the organism with parasitotropic chemical substances is termed by Ehrlich therapia sterilisans magna. 


\section{GENERAL THERAPEUTICS OF THE DISEASES OF THE ORGANS OF DIGESTION}

I. General Therapeutics of the Diseases of the Stomach

Pathology.-The diseases of the organs of digestion in our domestic animals are more varied and therefore more difficult to treat than those of man, especially the diseases of the stomach. While gastric ulcers and carcinoma of the stomach are generally rare in animals and the nervous dyspepsia so frequent in man probably does not occur at all, the therapeutics of stomach diseases in veterinary medicine is very complicated because the anatomical arrangement of the stomach is different in the several species of animals. The ruminants, especially, with their peculiar gastric apparatus, are in a separate class from the animals with a single stomach. Among the latter there exists again a considerable difference between the gastric digestion of the herbivora (horse), carnivora (dog, cat), and omnivora (swine). Other peculiarities are observed in the gastric apparatus of fowl.

The most important and the most frequent diseases of the stomach in animals are those occurring in connection with feeding (absolute and relative overfeeding, impaction of the rumen, acute tympanites, spoiled feed). The therapeutics is therefore first of all prophylactic (diet). In most cases the anatomical changes are confined to the mucous membrane (acute and chronic gastric catarrh, gastritis). However, in cattle all three layers of the stomach wall are very frequently affected (traumatic gastritis). In some animal species, parasitic diseases of the stomach are of importance (stomach-worm disease). Poisons also are not infrequently the cause of stomach diseases (irritants and irritant narcotic poisons). The stomach is also very often affected secondarily in general infectious and constitutional diseases.

Physiology.-The physiological processes concerned in gastric digestion are partly mechanical and partly chemical. Therefore, 
the therapeutics of diseases of the stomach will vary according to whether the disturbance of digestion originates in the muscular action and innervation of the stomach wall, in the gland secretions and chemical properties of the gastric juice, or in decomposition of the stomach contents. Accordingly, treatment may be required for the muscles, the glands, or the contents. From a therapeutic standpoint, the following physiological facts are of importance:

1. Mechanism of the Stomach.-In the horse, as well as in the other domestic animals with a single stomach (dog, hog, cat), the pylorus and cardia close immediately after a meal, and remain closed for a time. Then, the muscular layer in the stomach wall begins to contract, the pylorus is opened simultaneously, and the contents of the stomach are gradually emptied into the intestines. A part of the food and water ingested at this time, likewise of any medicines administered, pass directly into the intestines without detention in the stomach. This explains why medicines given by the mouth are sometimes surprisingly prompt in their action (cathartics, acetanilid, anthelmintics). The opening of the pylorus appears to be caused reflexly by the stimulant action of the gastric juice secreted and collecting in large amount. In addition, stimuli originating in the duodenum also operate to empty the stomach. In a similar way, other stimulants (alcohol, veratrum, oil of turpentine and spices) act reflexly through the intestines as well as the stomach and accelerate the opening of the pylorus and the emptying of the stomach.

The normal period of retention of food in the stomach differs greatly with the kind of food, the method of feeding and the animal species, as well as with the individual. in horses, after a small meal of oats, the passage of the contents of the stomach into the intestines begins in 2 to 3 hours and continues for more than 12 hours. Drinking water during or immediately after eating hastens the passage of the material in the stomach into the intestines. Water alone or fluid medicines, on the contrary, pass through the stomach of the horse very quickly; part in a few minutes. Exercise, especially trotting, retards the emptying of the stomach of the horse. 
In ruminants (cattle, sheep, goats), the mechanism of the stomach is especially complicated. Solid food and medicines pass into the rumen and reticulum and often remain, especially in the rumen, several days. Medicines should therefore not be administered to cattle in solid form if a prompt effect is desired. On the other hand, fluids or fluid medicines pass in part directly through the omasum and abomasum into the intestines, where they are rapidly absorbed and act promptly; the greater part, it is true, passes into the reticulum and thence into the rumen. Rumination results from the muscular activity of the rumen and reticulum and the pressure of the inferior abdominal muscles. The necessary conditions for the occurrence of rumination are a medium-full rumen and a certain amount of fluid in the rumen and reticulum. When the rumen is over-full or empty, or when the contents are dry, rumination ceases. The correction of these defects, especially supplying water when the contents of the rumen are dry, is of great therapeutic importance in disturbances of digestion. For the same reason, evacuating medicines which also stimulate gland secretions (arecoline, pilocarpine) are to be preferred to the mere muscle stimulants (eserine, barium chloride) in the treatment of impaction of the rumen.

The innervation of the stomach consists of the vagus and the sympathetic nerves and the automatic gastric centres-one each for opening and for closing the pylorus and the cardia. The motor centre (vagus) is situated in the corpora quadrigemina; the inhibitory centre (sympathetic) in the spinal cord. Contraction of the stomach wall may be produced by stimulation of the vagus nerve, or reflexly by stimulation of the gastric mucous membrane (stimulant stomachics). The secretion of gastric juice is influenced by the vagus.

2. Chemistry of Gastric Digestion.-The principal constituents of the gastric juice are hydrochloric acid, secreted by the glands of the fundus, and pepsin, secreted by the glands of the fundus and of the pylorus. Other ferments, rennet, a fat-splitting ferment, and a lactic acid ferment, are also present in the stomach. The function of the gastric juice is a double one. Of first impor- 
tance is the antiseptic and antizymotic action of the hydrochloric acid. In consequence of the presence of this substance the gastric juice is a natural protective agent against abnormal fermentative and putrefactive processes, and likewise against pathogenic bacteria. These facts are of the greatest therapeutic importance (prophylactic, causal, radical treatment of disease). It is also worthy of note that the antiseptic protective action of hydrochloric acid may be obtained from other acids, as lactic, acetic and phosphoric. The proteolytic or peptonizing action of the gastric juice, or of the pepsin, is only of secondary consideration therapeutically. Moreover, the digestion of proteids is not an exclusive physiological function of the stomach, but can be entirely accomplished by the pancreatic ferment (trypsin) and in part by the enzyme of the intestinal juice (erepsin). The proteolytic action of pepsin in the stomach occurs only when the contents are acid (hydrochloric, lactic, acetic acid). The optimum acidity is 0.2 per cent. If the acidity is less than 0.1 per cent. or more than 0.6 per cent. peptonization is inhibited. Accordingly, the average therapeutic dose of hydrochloric acid for the horse is 15 grams [one-half ounce], figuring the volume of the stomach to be 15 litres [15 quarts]. Absorption is relatively slight in the full stomach, especially in the stomach of the horse and in the first three stomachs of ruminants, and is limited to fluids or dissolved food and medicines. It is more rapid in the empty stomach of the fasting animal, especially in the stomach of dogs and in the abomasum of ruminants. These facts should be considered in administering medicines. On the other hand, of no special therapeutic importance are the different periods of gastric digestion in the horse (proteolytic pepsin digestion, amylolytic action of the saliva, mixed digestion) or the fact that in ruminants only maceration (rumen) and amylolytic (saliva) digestion occurs in the first three stomachs and proteolytic digestion in the fourth. More important is the fact that the contents of the stomach are arranged in layers and that no mixing takes place.

Therapeutic Methods.-Diseases of the stomach may be treated in several different ways. The methods most used are the dietetic, the medical, the mechanical, and the operative. 


\section{DIETETIC TREATMENT}

Since the greater portion of the diseases of the stomach in the domestic animals is due to abnormal properties in the food or to excessive feeding, the most important task of the veterinarian is the regulation of the diet. Sound, easily digested food, given in small quantity and at frequent intervals, is a primary requisite - green fodder, hay, carrots, molasses, etc., for herbivora; milk, raw meat, mucilaginous soups for dogs. In some cases, especially in colic of the horse, impaction of the rumen in cattleand acute gastric catarrh in the dog, it is advisable to withhold food entirely. Dogs must be deprived of water also sometimes; in morbid conditions of the stomach these animals drink large quantities of water and persistent vomiting results. These methods, by resting the affected parts, bring about complete recovery in many cases. Work animals should have bodily rest in addition, because severe exertion or exercise reduces or entirely suppresses gastric digestion.

\section{MEDICINES. (STOMACH REMEDIES. STOMACHICS)}

Synonyms: Digestives in a restricted sense, peptics, ruminatorics, anti-dyspeptics, antemetics, antacids, neutralizants, absorbents, suppletives; stimulants to the appetite, improvers of the digestion, acid-combating, stimulants to rumination.

Classification.-According to their action and composition, the stomachics are divided into the following groups:

(a) Physiological Stomachics.-These are normally contained in the gastric juice and when decreased in quantity or absent are supplied artificially. They are: acidum hydrochloricum and pepsinum. It is best to administer both together, as it is not practicable to determine in animals if one or the other is absent. They can be used in the treatment of all diseases of the stomach and in all disturbances of digestion in the course of febrile and general diseases with the exception of the rarely occurring gastric ulcers (calves). In veterinary practice hydrochloric acid is most commonly used.

The dose of hydrochloric acid for cattle is $15-30$, 5 ss to $5 \mathrm{j}$; horses, 10-20, 3 ijss to $3 \mathrm{v}$; calves, sheep, goats and swine, 1-2, 
mxv to $\mathrm{xxx}$; dogs, cats and fowl, 0.1-0.5, mi to vij. Of pepsin, the dose for horses is $5-10,3 \mathrm{j}$ to $3 \mathrm{ijss}$; dogs, $0.1-1$, grs. jss to xv. Phosphoric acid or lactic acid may be used in place of hydrochloric acid.

(b) Saline Stomachics stimulate the secretion of hydrochloric acid, dissolve collections of mucus upon the gastric mucous membrane, and at the same time combat fermentation. The most important are sodii chloridum, sodii bicarbonas, sodii sulphas and *Carlsbad salts. ${ }^{1}$ They are the best remedies for chronic gastric catarrh.

The dose of sodium chloride, sodium bicarbonate, sodium sulphate and Carlsbad salts for cattle is $50-100$, 5 jss to iij; horses, 25-50, 3 vj to xij; sheep and goats, $2-5$, grs. $x x x$ to $3 \mathrm{j}$; dogs, 1-2, grs. $\mathrm{xv}$ to $\mathrm{xxx}$.

(c) BitTer Stomachics (Amara).-In disturbances of gastric digestion these improve the appetite, increase the secretion of gastric juice, stimulate reflexly the contraction of the stomach wall and prevent or check fermentation. The alleged negative results obtained in experiments with bitter stomachics on healthy individuals and outside of the animal body do not disprove their favorable effect upon disturbances of digestion. Gentiana and aloe are the most important of those used in veterinary medicine. Others are: *condurango, cinchona, *absinthium, taraxacum, calumba, *centaury, *menyanthes, *centaurea, quassia, *cetraria, *achillea, [nux vomica], and strychnina. As a rule, the amara are administered in small doses.

The dose of aloes as a stomachic for cattle is $5-10,3 j$ to ijss; horses, sheep and goats, $2-5$, grs. $x x x$ to $3 j$; dogs $0.1-0.5$, grs. jss to vij. Of gentian, absinthe and the other vegetable amara, except nux vomica and strychnine, the dose for cattle is $25-50,3 \mathrm{vj}$ to xij; horses, 10-25, 3 ijss to vj; sheep and goats, 2-5, grs. $x x x$ to $3 j$. The tinctures of these drugs are usually administered to dogs, the

* [Not official in the U. S.]

1 [The formula for artificial Carlsbad salts, Sal Carolinum factitium, is as follows: Sodium sulphate 22 parts, sodium bicarbonate 18 parts, sodium chloride 9 parts, and potassium sulphate 1 part.] 
dose being 5 to 10 drops. Strychnine is also administered [in Germany] in the form of the tincture to dogs, calves, and lambs. [In the United States, where the tincture of strychnine is not official, tinctura nucis vomicæ is prescribed as a bitter stomachic in the following doses: for horses and cattle, $4-25,3 \mathrm{j}$ to vj; dogs, $0.3-1$, nuv to xv.] Oxgall, fel bovis, is obsolete; it is also harmful, as it precipitates pepsin. Condurango is alleged to be a specific for gastric carcinoma.

In the old therapeutics there were superfluous subclasses of the bitter stomachics: Amara vera or pura (gentian), A. mucilaginosa (cetraria), A. aromatica (absinthe), A. salina (magnesium sulphate), A. adstringentia (cinchona), A. tetanica (strychnine), A. cathartica (aloe).

(d) Aromatic Stomachics.-These stimulate the activity of the glands of digestion, the appetite and the peristalsis of the stomach. To this class belong rheum, calamus, anisum, fœniculum, carum and *fuctis juniperi; also sinapis alba and nigra, *angelica, valeriana and aurantii amari cortex.

The dose of rhubarb as a stomachic for horses and cattle is 10-25, 3 ijss to vj. Of the other aromatics mentioned, the dose for cattle is $25-50,3 \mathrm{vj}$ to xii; horses, $10-25$, 3 ijss to vj; sheep and goats, 2-5, grs. $x \times x$ to $3 \mathrm{j}$; dogs, 0.5-2, grs. vij to $\mathrm{xxx}$. Rhubarb is given to dogs in the form of the tincture, the dose being $4,3 \mathrm{j}$.

(e) Stimulant Stomachics.- The most important of these is alcohol, which is also antiseptic; in addition, the group includes the pungent spices: piper and capsicum, likewise the new remedy, *orexin. All these substances cause hyperæmia of the gastric mucous membrane, abundant secretion of gastric juice, increased peristalsis and a more rapid emptying of the stomach. They are contra-indicated in severe affections of the stomach.

The dose of alcohol as a stomachic for horses and cattle is 25-50, $3 \mathrm{vj}$ to $\mathrm{xij}$; dogs, 2-5, mxxx to $3 \mathrm{j}$. The dose of the peppers for cattle is $10-20,3$ ijss to $\mathrm{v}$; horses, $5-10,3 \mathrm{j}$ to ijss; swine, $1-2$, grs. $\mathrm{xv}$ to $\mathrm{xxx}$; dogs, $0.1-0.2$, gr. $\mathrm{j}$ to iij.

(f) Disinfectant Stomachics.-These are used in fermenta- 
tion and decomposition of the stomach contents, also in infectious gastric catarrh and against parasites (stomach worms, larvæ of the bot $\mathrm{fly}$ ). To this class belong acidum hydrochloricum, creolin, creosotum, naphthalenum, bismuthi subnitras, quinina, iodum, oleum terebinthinæ and carbonei disulphidum.

Of these, the most important in veterinary medicine are hydrochloric acid and creolin. Both may be given to cattle and horses in doses of 10-25, 3ijss to vj; sheep and goats, $0.5-1$, mivij to $\mathrm{xv}$; dogs, 0.1-1, mj to $\mathrm{xv}$; fowl, 0.1-0.25, mj to iij. The dose of carbon disulphide (bot fly larvæ) for horses is $10,3 \mathrm{ijss}$, repeated four times at intervals of one hour.

(g) Narcotic Stomachics (Antemetics) are prescribed in painful affections of the stomach and in persistent vomiting. The sedative remedies are opii pulvis, cocainæ hydrochloridum and menthol.

Opium is most frequently used. Dose for horses and cattle, 5$25, \mathrm{zj}$ to $\mathrm{vj}$; dogs, $0.1-0.5$, grs. $\mathrm{j}$ to vij. [Of the tinctura opii, the dose for horses and cattle is $30-60$, $5 \mathrm{j}$ to $\mathrm{ij}$; dogs, $0.2-2$, niiij to $\mathrm{xxx}$.]

(h) Antacids (Acid-neutralizing Stomachics, Absorbents). -These combine with abnormal acids or an excess of normal acids in the stomach and neutralize excessive amounts of hydrochloric and lactic acids or abnormal acids (fatty acids, carbonic acid, poisonous acids). To this group belong sodii bicarbonas, potassii bicarbonas, soap, liquor calcis, calcii carbonas præcipitatus, *sodium, *potassium, magnesii oxidum, magnesii carbonas, aqua ammoniæ and ammonii carbonas.

Soap is an important absorbent for carbolic acid especially; lime water for oxalic and sulphuric acids, and the other substances mentioned above for hydrochloric, lactic and the fatty acids, etc. The dose is ad libitum except for sodium, potassium, and the oxide and carbonate of magnesia. Of the latter, the dose for horses and cattle is $5-25$, $3 \mathrm{j}$ to $\mathrm{vj}$; sheep and goats, 1-2, grs. $\mathrm{xv}$ to $\mathrm{xxx}$; dogs, $0.2-1$, grs. iij to $\mathrm{xv}$.

(i) RuMINATORICS.-The drugs that stimulate rumination are emetics, muscle stimulants, and drastic stimulants of the gastric 
mucous membrane. Those most commonly used are antimonii et potassii tartras, veratrum, ipecacuanha, alcohol, *veratrina, arecolinæ hydrobromidum, physostigminæ salicylas or sulphas [eserine], *eseridin tartras, pilocarpinæ hydrochloridum or nitras, oleum terebinthinæ, *tabacum, colchici semen, ammonii carbonas and aqua ammoniæ.

In cattle practice, the most important ruminatorics are tartar emetic, veratrum, veratrin and arecoline. Doses: Tartar emetic, for cattle, 10-20, 3 ijss to $\mathrm{v}$. Powdered veratrum or the tincture, for cattle, $5-10,3 i$ to ijss; sheep and goats, $0.5-2$, grs. vij to $x x x$. Oil of turpentine, cattle, 25-50, $3 \mathrm{vj}$ to $\mathrm{xij}$; sheep and goats, 2-5, noxx to $3 \mathrm{j}$. Veratrin and eserine, for cattle, 0.1-0.2, grs. $11 / 2$ to 3. Arecoline, for cattle, up to 0.05 , gr. $3 / 4$.

In carnivora, an emetic not rarely acts as a good stomachic.

\section{MECHANICAL TREATMENT}

In ruminants, the rumen is situated in the left flank and is in contact with the abdominal wall when greatly distended. It can therefore be very easily massaged. Massage, or kneading, of the rumen is of the greatest importance in the treatment of digestive disturbances in cattle, especially impaction of the rumen, acute tympanites and all atonic conditions of the musculature of the rumen. It stimulates reflexly. the muscular contractions of the rumen, which under normal conditions occur about twice a minute. In dogs, the stomach when full also lies close to the abdominal wall and can also be massaged. It is not possible, however, to massage the stomach of the horse, because even when full it can not be palpated through the abdominal wall.

Of the other mechanical methods, the most important is the introduction of the œsophageal sound in ruminants and of the stomach tube in horses to remove gas and the fluid contents of the stomach. Electrotherapy and hydrotherapy are not usually employed on animals. The emetics also act in a mechanical manner in emptying the stomach of its contents (see p. 34).

[For several years, American veterinarians have been using soft rubber tubes to remove gas from the stomach of horses and to 
wash out the stomach. There are two types of these tubes: one is passed through the mouth and the other is introduced into the œsophagus by way of the inferior meatus of one of the nasal cavities, a flexible rattan stilet being inserted into the tube before it is passed, and withdrawn after the tube is in position.

In the small animal clinic at Copenhagen, Prof. Hansen washes out the stomach of dogs, as well as other parts of the alimentary canal, by injecting per rectum a 0.9 per cent. solution of sodium chloride at body temperature, the posterior part of the body being elevated. After 2 to 3 litres are injected into small dogs and up to 9 litres in large dogs, the fluid, mixed with alimentary matters, is vomited. The injection is continued until the fluid is vomited clear. The method has been in use in the small animal clinic at the University of Pennsylvania for the last two years. In toxic, hemorrhagic, and catarrhal enteritis and in icterus the results are very satisfactory.]

\section{OPERATIVE TREATMENT}

In acute tympanites of ruminants puncture of the rumen is in most cases necessary to remove the gas. The rumen may at the same time be irrigated with water or fluid medicines introduced through the cannula. Gastrotomy is resorted to to remove foreign bodies from the stomach of dogs (stones, coins, balls, corks) and cattle (metallic foreign bodies in the reticulum, abnormal collections of food and poisonous plants in the rumen). Finally, diseases of the stomach may be treated indirectly by operation, as by the extraction of diseased teeth in the dog.

\section{EMETics}

Synonyms: Vomitives, vomitories, nauseosa, nauseotics.

Method of Action.-Vomition results, according to some, from an active, primary contraction of the stomach; according to others, from the passive compression of the stomach by the convulsive contraction of the inferior abdominal muscles and the diaphragm. The cardia being relaxed at the same time and the pylorus closed, 
the contents of the stomach are forced upward and forward. Vomiting is under the control of the centre of vomition (a paired centre situated in the region of the calamus scriptorius). This centre can be directly stimulated through the blood and indirectly by stimulation of peripheral parts of the body, especially the stomach. Emetics which can cause vomiting by acting through the blood and stimulating the vomiting centre without necessarily coming in contact with the stomach are called central or general emetics. Apomorphine is a good example of this variety. Those that cause vomiting reflexly by irritating the gastric mucous membrane are called local or topical emetics. Examples are copper sulphate, zinc sulphate, tartar emetic. Some emetics operate both ways, e.g., em tin. - Pathology teaches that vomiting may also be caused reflexly by the irritation of numerous peripheral parts outside of the stomach, as the mucous membrane of the base of the tongue, the throat, cesophagus, intestines, uterus, pelvis of the kidney and gall ducts, and the terminations of the auricular branch of the vagus (otorrhoea). Centric vomiting may also occur in different diseases of the brain and in uræmia. Finally, stimuli applied to centres related to the centre of vomition appear to pass readily over the latter. Hydrocyanic acid, which stimulates the respiratory centre, may exert a similar action upon the centre of vomition. The drugs that have a specific stimulant effect upon the vagus centre (digitalis, squill, strophanthus) may also stimulate the vomiting centre.

The effects of vomiting upon the stomach and other organs of the body are very extensive. In the first place, the stomach, cesophagus, pharynx and to a certain extent the buccal cavity are emptied. The peristaltic movement of the stomach wall is increased through the contraction of the gastric musculature. Intestinal peristalsis is also increased, being stimulated reflexly by the contraction of the stomach. Emetics which exert an especially pronounced action upon the intestines are called emetocathartics. A further effect of vomiting is the reflex stimulation of the secretion of the stomach and intestinal juices and of the bile. The contraction of the inferior abdominal muscles exerts 
compression upon all of the organs in the abdominal cavity. In consequence of this, the elimination of bile and pancreatic secretion is accelerated and a certain amount of blood is expressed out of the large vessels and organs of the posterior part of the abdomen, these organs becoming anæmic while the neighboring organs become hyperæmic. During vomiting the pulse and respiration are accelerated, metabolism is increased, and there is also at the same time increased activity of the sweat glands and the mucous glands of the bronchial membrane. The forced expiration occurring during vomiting increases the expectoration of secretions from the bronchi and lungs. The nervous system in general is stimulated, but it is questionable if the irritability of the muscles is decreased. Finally, the nausea that precedes voling has an alterative effect upon the gastric nerves. Emetics which produce a pronounced and long-continued nausea are called nauseating emetics (apomorphine).

Uses.-The diverse and wide-spread effects of emetics demonstrate that the use of emetics has unwisely been discredited. In veterinary medicine, they are indispensable in the treatment of certain diseases; in other diseases practical experience, in confirmation of the aforementioned physiological effects, has shown that they are of decided value. The most important indications for the employment of emetics are the following:

1. The removal of excessive quantities of food, foreign bodies, and poisons from the stomach and osophagus.

2. To empty the stomach of decomposing or fermenting food, and of collections of mucus in gastric catarrh.

3. Licking-disease of cattle and wool-eating of sheep are most promptly cured by emetics. This is also true of other forms of depraved appetite (eating of sand by horses).

4. The removal of stasis of bile (icterus).

5. The removal of infectious material from the stomach in infectious diseases. For example, in the early stages of canine distemper and erysipelas of swine an emetic is indicated. In such cases emetics act as alteratives (so-called abortive treatment). 
6. Expectoration of inflammatory products from the respiratory tract, especially in young dogs affected with distemper.

7. The removal of blood stasis in the liver and other organs in the posterior part of the body.

Emetics are contraindicated in very weak animals, gastric ulcers, inflammation of the stomach, advanced pregnancy, herniæ and certain diseases of the heart and blood vessels (aneurism).

Emetics.-1. Apomorphinæ hydrochloridum. This is the best emetic for dogs. The dose subcutaneously is $0.002-0.01$, gr. $1 / 40$ to $1 / 8$. As a specific against licking-disease in cattle and wool-eating in sheep, it is used subcutaneously in doses of 0.1-0.2, grs. jss to iij. Cats as a rule require ten to twenty times the dose for the dog. Apomorphine usually does not cause vomiting in swine.

2. Veratrina. The most important emetic for swine. Dose: $0.02-0.03$, gr. $1 / 4$ to $1 / 3$, in alcohol, subcutaneously. In place of veratrin subcutaneously, veratrum may be administered per os or per rectum. Dose for swine, $0.5-2$, grs. viij to $x x x$; dogs, $0.05-0.2$, grs. $3 / 4$ to iij.

3. Ipecacuanha. An emetic for cats, dogs, and swine. Dose for dogs and swine, 1-3, grs. xv to xlv; cats, $0.25-0.75$, grs. iij to $\mathrm{x}$. [Vinum ipecacuanhæ, on account of its stimulating properties, is a valuable emetic in canine and feline practice. Dose for dogs, 1-4, IएXv to $3 \mathrm{j}$; cats, one-half the quantity.]

4. Antimonii et potassii tartras. Tartar emetic, an emetocathartic; therefore suitable only for robust animals (swine, large dogs). Dose for swine, $1-2$, grs. $x v$ to $x x x$; dogs, 0.1 to 0.3 , grs. jss to jv. Vinum antimonii is also useful and is given to dogs in teaspoonful to tablespoonful doses.

5. Cupri sulphas. A specific emetic in phosphorus poisoning. It unites with the phosphorus to form phosphor-copper, which is mat poisonous. Dose for dogs, $0.1-0.3$, grs. jss to jv.

Emetics like mustard, table salt, ammonium carbonate, and zinc sulphate, which were formerly much used by the laity, are now prescribed only in emergency.

For antemetics, see the chapter on stomachics. 


\section{iII. General Therapedtics of the Diseases of the INTESTINES}

Pathology.-The diseases of the intestines most frequently requiring treatment are acute and chronic intestinal catarrh, very often associated with gastric catarrh; constipation; colic, with its numerous anatomical causes (impaction, displacements, etc.); the different forms of enteritis, and helminthiasis (tape worms, round worms). More rarely, foreign bodies and neoplasms are present in the intestines, which are also affected secondarily in many of the infectious diseases. The incurable chronic intestinal catarrhs, which are especially frequent in cattle and dogs, and some cases of chronic gastric catarrh are not merely a superficial disease of the epithelium but a severe parenchymatous affection of the intestinal glands, accompanied by atrophy of the latter and formation of new connective tissue; the pathological processes being analogous to those of chronic nephritis. These circumstances explain the fruitlessness of treatment.

Physiology.-The cause of the normal peristaltic movements of the intestines is the reflex stimulation of the intestinal nerves by the intestinal contents. In addition to a number of automatic centres in the intestinal wall (plexus myentericus), there are also constrictor and dilator nerve apparatuses. The accelerator nerve is the vagus (cranial); the inhibitory nerve is the splanchnic (spinal). Stimulation of the vagus causes intestinal contraction. Stimulation of the splanchnic (morphine) retards or inhibits intestinal contraction; paralysis of the splanchnic (atropine) increases the intestinal movements. The average time required for food to pass through the digestive tract is 3 to 4 days for horses and cattle, sometimes double this period and more for the latter; for dogs 12 to 15 hours; swine 24 to 36 hours.

The reaction of the contents of the anterior part of the intestines is acid, because of the presence of the acid from the stomach. The small intestines are therefore relatively poor in bacteria. In the large intestines, and in herbivora even in the ileum, the reaction is alkaline in consequence of the neutralization of the gastric 
juice by the bile, pancreatic juice and intestinal secretions, all of which are alkaline. Intestinal digestion takes place principally in the small intestines. In the large intestines a further digestion takes place, and here also occur, especially in the cæcum, in addition to the resorption of the fluid and dissolved substances, fermentative and putrefactive processes (indol, skatol, phenol, cresol, fatty acids, amido acids, ammonia, carbon dioxide, hydrogen, hydrogen sulphide, and other products of the decomposition of albumin and cellulose). In contrast with the small intestines, the large intestines, on account of the alkaline reaction and the absence of oxygen, are a place for the incubation of anaërobic bacteria (colon bacillus, bacillus putrificus-decomposers of albumin). The bile and the pancreatic juice are the most important of the digestive secretions, the intestinal juice possessing only a slight amylolytic and at times a proteolytic action. The bile possesses antitoxic and antiseptic properties (immunization in infectious diseases, removal of poisons from the blood). It also prepares the fats for absorption in the intestines and operates as a stimulant to peristalsis of the large intestines. The pancreatic juice contains proteolytic (trypsin), amylolytic and fat-splitting ferments.

In regard to intestinal absorption it is to be noted that the nutrient substances in solution and the fats are absorbed by the activity of the intestinal epithelium and the contraction of the villi, assisted by the amœboid functions of the leucocytes (phagocytosis). The water-soluble substances are also taken up by diffusion. When the intestinal epithelium is desquamated over a considerable area, as in catarrh, or when the intestinal villi are paralyzed, as in inflammation, then absorption is distributed or entirely suppressed.

Therapeutic Methods.-The diseased intestine may be influenced in several ways. The mucous membrane may be treated directly with demulcents, narcotics, astringents, antiseptics or stimulants. The glands may be acted upon by pilocarpine or arecoline; the muscles by eserine or barium chloride; the nerves by morphine. Treatment of the intestinal contents is also very impor- 
tant and is accomplished by dieting and by the administration of cathartics, intestinal styptics, disinfectants and anti-ferments. The principal therapeutic methods are similar to those used in the treatment of diseases of the stomach, namely: dietetic, medical (cathartics, styptics), mechanical and operative.

\section{DIETETIC TREATMENT}

Fasting, or a diet of non-irritating, easily digested food, will serve, more than anything else, to alleviate a catarrhal or inflamed mucous membrane. Green fodder, bran, or linseed meal is best for horses; raw meat, milk, eggs, and mucilaginous soups for dogs. When catarrh is present in the intestines, the stomach is frequently healthy and the appetite is very good. This must be remembered so that overfeeding will be avoided. Taking a large quantity of food at one meal is not to be permitted.

\section{CATHARTICS. LAXATIVES}

Synonyms: Purgatives, drastics, lenitives, eccoprotics, aperients, aperitives, evacuants, peristalties, osmotics.

Classification.-For the purposes of general therapeutics it is best to divide the cathartics into three groups according to the intensity of their action: mild, medium and strong. Although these different degrees of action may be obtained with the same cathartic by simply varying the dose, and although the intensity of action may vary with the species, yet for practical reasons and because it affords a better conception of the large number of cathartics, it is desirable to classify these drugs in the three following groups:

(a) Drastics, very powerful cathartics, which operate on the horse in doses of a few grams, decigrams and even centigrams. This group includes arecoline, eserine, pilocarpine, tartar emetic, calomel, podophyllum, croton oil, gamboge, jalap, colocynth and barium chloride.

(b) Purgatives, cathartics of medium strength, laxatives in the restricted sense. In this group are included aloes and the saline 
purgatives: sodium sulphate, potassium sulphate and magnesium sulphate; also, fructus rhamni catharticæ, frangula and cascara sagrada.

(c) Laxatives or Lenitives, mild cathartics, which must be given in very large doses and most of which do not operate upon horses and cattle. This group includes castor oil, senna, sulphur, manna, tamarind, syrup, honey, glycerin, and the fixed oils.

Theories Regarding Catharsis.-The question of how and in what manner catharsis is brought about after the administration of a cathartic has been answered in many different ways. A number of theories have been proposed by Liebig, Buchheim, Aubert, Voit, Hay, Brieger, Radziejewski, and others. From these we learn that catharsis is not a simple process but a very complicated action resulting usually from several factors operating together.

(a) According to Liebig, the neutral salts operate principally in a purely physical manner, i.e., in accordance with the laws of osmosis. Water passes from the blood, which is poor in salts, into the intestines, which contain a large amount of salt. The "animal membrane" separating the blood from the intestinal contents is the intestinal mucous membrane. If a solution of Glauber's salt or epsom salt is placed in an isolated loop of the small intestine the latter will be filled to distention in a few hours with a clear yellow, alkaline fluid. At the same time the mucous membrane will retain its normal pale color. The fluid which passes from the blood into the intestines operates as a stimulant to peristalsis and diarrhoea occurs.

(b) On the other hand, Buchheim asserts that the neutral salts produce catharsis by retarding or suppressing absorption of intestinal fluids in consequence of their slight diffusibility. The more slowly and least diffusible salts, sodium sulphate, potassium sulphate and magnesium sulphate, therefore, possess a cathartic action in contrast with the rapidly and readily diffusible sodium chloride. Other cathartics, especially the drastics, probably retard absorption by paralyzing the intestinal epithelium and villi.

(c) Peristalsis is reflexly stimulated and accelerated through the direct stimulation of the nerves in the intestinal mucous mem- 
brane by most of the cathartics, including calomel, aloes, rhubarb, sulphur and especially the neutral salts. The latter operate in at least three different ways. Proof of their stimulant action is found in the fact that very dilute solutions, which could not generate an osmotic stream, produce diarrhœea. Radziejewski studied experimentally the influence of cathartics upon peristalsis by means of intestinal fistulæ in dogs and found that the peristaltic movements of the intestines and the flow of fluid through the fistulæ were considerably increased after the administration of laxatives. Intestinal peristalsis may be stimulated reflexly from the stomach by some drugs; croton oil, for example, will produce diarrhœa while it is yet in the stomach. Acceleration of peristalsis causes a more rapid discharge of the intestinal contents. On account of the decreased absorption of the intestinal fluids, the discharges are thin and fluid; they are the unchanged contents of the small intestines.

(d) Increased secretion of the intestinal glands with increase of the intestinal fluids and acceleration of peristalsis. The most important representatives of this group are arecoline and pilocarpine, specific gland stimulants. The neutral salts also stimulate the intestinal glands during their elimination from the blood, thus making their action a four-fold combination. Intestinal secretion is also stimulated reflexly by those drugs which irritate the mucous membrane, especially the drastics.

The intestinal fluids, including the secretions of the mucous glands, Lieberkühn's and Bruner's glands, the pancreas and liver, can only be increased in sufficient amount to produce diarrhœa when the blood is rich in water. As Hay has pointed out, fluid bowel discharges are not produced by the neutral salts, even in large doses, when animals have been without water for several days and have received only dry feed. This observation agrees with the well-known experience of veterinarians that a horse or cow which has received a cathartic must be permitted to drink a large quantity of water in order to accelerate the action of the drug and increase its effect.

(e) Transudation and exudation of blood-serum as a cause of 
collection of fluid and increased peristalsis occur as the result of the action of any of the irritant cathartics which produce hyperæmia, catarrh or inflammation in the intestinal mucous membrane. In this group are included croton oil, tartar emetic, colocynth, jalap, gamboge and other drastics. These drugs should not be prescribed in acute inflammatory conditions of the intestines.

(f) Intestinal tetanus, with rapid expulsion of the intestinal contents, is the cause of the cathartic action of eserine, nicotine and barium chloride and partly also of arecoline and pilocarpine.

(g) Paralysis of the splanchnic, the inhibitory nerve of the intestines, results in the acceleration of peristalsis. Atropine acts in this manner.

(b) Lubrication of the intestinal walls mechanically accelerates the passage of the intestinal contents. This is the mode of action of the fixed oils, but in part only of castor oil.

Results of Catharsis. - Scarcely any other action of drugs is accompanied by so many local and general effects as catharsis. To begin with, the intestinal canal is emptied of whatever it may contain: normal contents, fermented or decomposed food, calculi, concrements, poisons, inflammatory products, parasites or infectious materials. Secondly, peristalsis is accelerated and the secretions of the intestinal glands increased. Some cathartics, as aloes, rhubarb, colocynth and podophyllum, at the same time increase the secretion of bile; these are called the cholagogue cathartics. The withdrawal of fluid from the blood into the intestines has the effect of condensing the blood, in consequence of which the blood absorbs fluids from the tissues (hydragogue cathartics). The volume of the blood is decreased by the loss of fluid and the blood-pressure is lowered. The distribution of blood is materially changed and the body temperature is lowered. While the intestinal mucous membrane and the other abdominal organs are rich in blood, especially after the administration of drastics, the more distant organs, as the brain, lungs and skin, are correspondingly poor in blood, the blood being diverted from the latter to the intestines. The activity of the kidneys is at first increased by some cathartics; for example, diuresis is increased a few hours 
after the administration of the neutral salts. Usually, however, the secretory activity of the kidneys is reduced by cathartics. This is also the case with the sweat glands. Another effect of the increased secretion of the intestinal glands is the elimination of infectious material, metabolic products and poisons from the blood. Emptying the intestinal canal withdraws nourishment and more or less disturbance of the appetite, and digestion is also associated with catharsis. Finally the drastics, like cutaneous irritants, by irritating the sensory nerves in the intestinal mucous membrane exert reflexly a general stimulant action upon the nervous system, affecting the animal as well as the vegetative functions. In this action the principle of counter irritation plays an important rôle.

Use of Cathartics.-On account of their manifold action cathartics are employed in the treatment of a large number of diseases.

1. Constipation and depressed peristalsis in the course of intestinal catarrhs, constipation-colic in horses, fecal stasis in dogs, and high fever with suppressed intestinal secretions.

2. Overfeeding of herbivora; also the presence of foreign bodies, poisons, parasites, bacteria, and toxins in the intestines.

3. Diarrhoeas and gastric catarrhs due to fermenting foodmasses or infectious materials in the intestines. These conditions occur in dysentery, canine distemper, influenza of horses, swine erysipelas and fowl cholera. Calomel is especially indicated in these conditions because it is also disinfectant.

4. Liver diseases, especially catarrhal icterus.

5. Hyperæmia and inflammation of the lungs, pleura, brain, spinal cord, skin (urticaria), pododerm (laminitis; arecoline) and udder (mastitis). In these conditions cathartics are used for their derivative and antiphlogistic effects.

6. Dropsical conditions; also to assist the resorption of fluid and solid exudates.

7. Acute and chronic nephritis, and uræmia. The compensatory increase in the secretions of the intestinal glands relieves the kidneys in these conditions. 
8. In obesity, gout and increased sexual impulse to withdraw nourishment and reduce bodily strength.

9. Prophylactically, against parturient apoplexy and azoturia.

10. To prepare cryptorchids for operations; also animals for intestinal operations (laparotomy).

Cathartics are contraindicated in severe non-infectious inflammation of the stomach or intestines, in very weak, anæmic animals, in advanced pregnancy, in peritonitis (extension of the inflammation over the whole peritoneum by the increased peristalsis), and in prolapse of the rectum. When a cathartic is absolutely necessary in any of these conditions castor oil or calomel should be used. On account of the elimination of cathartics in the milk, neutral salts or other indifferent substances should be given to milk cows instead of aloes.

The Individual Cathartics.-1. Physostigminæ salicylas. Physostigminæ sulphas. Eserine. An important cathartic for the horse in constipation colic. Operates within a half hour. Also used in combination with pilocarpine. Contraindicated in excessive distention and paralysis of the intestines, engorgement of the stomach, tympanites, spasmodic colic, pregnancy and dyspnœe. Dose for the horse, $0.05-0.1$, gr. $3 / 4$ to jss, subcutaneously.

2. Arecolinæ hydrobromidum. Arecoline combines the action of physostigmine (eserine) and pilocarpine, is cheaper, more stable and is effective in smaller dose. Especially valuable in laminitis, colic and inflammation of the brain. Dose for the horse, $0.05-0.08$, gr. $3 / 4$ to $\mathrm{j}$.

3. Aloe. Aloes. A cathartic for horses and cattle in impaction colic, chronic intestinal catarrhs, inflammation of the brain and laminitis; also a cholagogue. It has the disadvantage of operating slowly: 18 to 36 hours after administration. Dose for the horse, 25-50, $3 \mathrm{vj}$ to xij; for cattle, $40-60,3 \times$ to $5 \mathrm{ij}$. [Dose of aloin for the horse, 8-12, $3 \mathrm{ij}$ to ij; produces less dulness, nausea and griping than aloes and is more prompt in action.]

4. The neutral salts: Sodii sulphas, magnesii sulphas, and *sal Carolinum factitium (artificial Carlsbad salts). Mild and slow acting cathartics; used alone or to assist other cathartics, as aloes, 
arecoline and eserine, in constipation and colic, and to produce a derivative action. They are at the same time diuretics and cholagogues. For cattle, they are in general somewhat too weak, and in horses their action occurs only after 1 or 2 days. They are too bulky for dogs. The dose of sodium sulphate, magnesium sulphate and Carlsbad salts for horses is $250-500, \mathrm{lb}$. ss to $\mathrm{j}$; for cattle, $500-1000,1 \mathrm{lb}$. j to ij; sheep and goats, $25-50,3 \mathrm{vj}$ to xij; swine, $25-50$, $3 \mathrm{vj}$ to xij; dogs, 10-25, 3 ijss to vj; cats and fowl, 2-5, 3 ss to j.

5. Hydrargyri chloridum mite. Calomel. The most important cathartic for dogs and swine; best intestinal disinfectant; may also be given in inflammatory and ulcerous conditions of the intestines; should not be administered to cattle and should be used on horses with caution. Dose as a laxative, for swine, $1-4$, grs. $x v$ to $3 \mathrm{j}$; dogs, 0.2-0.4, grs. iij to vj; cats, 0.1, grs. jss; fowl, 0.05-0.2, grs. $3 / 4$ to $\mathrm{iij}$.

6. Antimonii et potassii tartras. Tartar emetic. A good laxative for horses and cattle, especially in colic and parturient apoplexy and to produce a derivative action upon the intestines; should not be given to weak animals. Dose for horses, $2-10$, 3 ss to ijss; cattle, 10-20, 3 ijss to v; sheep 0.5-2, grs. vij to xxx; calves, $0.3-0.5$, grs. iv to vij.

7. Oleum ricini. Castor oil. A mild cathartic for all the domestic animals, but especially for dogs; may also be given in inflammation of the intestines; contraindicated in phosphorus and cantharides poisoning. Dose for dogs, $15-60$, 5 ss to ij; horses, 250-750, Oss to jss; cattle, 500-1000, Oj to ij; foals, calves, sheep and goats, 50-250, 兮 jss to viij; swine, $50-100$, 5 jss to iij; cats and fowl, 10-30, 3 ijss to 5 i.

[8. Oleum lini. Linseed oil. In the United States linseed oil is more commonly used as a cathartic for horses and cattle than castor oil. Dose same as castor oil.]

9. Oleum tiglii. Croton oil. The most powerful drastic cathartic for the horse; other drugs are more desirable; contraindicated in all cases of intestinal inflammation and for weak and sensitive animals. Dose for horses, $0.6-1.5$, wx to $\mathrm{xx}$; cattle, 1-2, mexv to $\mathrm{xxx}$; dogs, $0.07-0.12$, wij to ij. 
10. Rheum. Rhubarb. A very mild cathartic for dogs and cats, the catharsis being followed by constipation. Also a stomachic and cholagogue. Dose for dogs, $5-15,3 \mathrm{j}$ to iij; cats and fowl, $2-5,3$ ss to 3 j. [Of Tinctura rhei, the cathartic dose for the $\operatorname{dog}$ is 1 to $2 \frac{1}{2}$ tablespoonfuls; cats, $1 / 4$ to 1 teaspoonful.]

11. Senna. A cathartic for the small domestic animals, especially swine. Dose for swine, $10-25,3$ ijss to vj; dogs, $5-15,3 \mathrm{j}$ to iij ; cats, $2-5,3$ ss to j; fowl, 1-2, grs. xv to xxx.

12. Cambogia. Gamboge. A drastic cathartic for swine and dogs. Dose for swine, $2-4,3$ ss to $j$; dogs, $0.2-1$, grs. iij to xv.

13. Jalapa. Jalap. A drastic cathartic, cholagogue and hydragogue for the small domestic animals. Dose for swine, 5-15, $3 \mathrm{j}$ to iij; dogs, 0.5 to 4 , grs. vij to $3 \mathrm{j}$.

14. Podophyllum. A powerful drastic cathartic and cholagogue. The dose should be gauged cautiously. Dose for dogs, $0.1-0.25$, grs. jss to iijss.

15. Colocynthis. Colocynth. Action same as podophyllum. Dose for swine, $8,3 \mathrm{ij}$; dogs and cats, $0.2-2$, grs. iij to xxx.

16. *Syrupus rhamni catharticæ (N.F.). Action the same as podophyllum and colocynth. Dose for dogs, 1 to 2 tablespoonfuls.

17. Tabacum. Tobacco. Used formerly in colic in horses in the form of smoke clysters.

18. *Barii chloridum. Quickest acting cathartic when injected intravenously. Dose for the horse, $0.5-1.25$, grs. vij to xviij, the latter for large horses. A heroic and very toxic colic remedy; must be used with caution in heart weakness (given in fractional doses, $0.25-0.5$, grs. iij to vij, at 15-minute intervals).

19. Sulphur sublimatum, S. præcipitatum, S. lotum. Sulphur. Rarely used now as a cathartic. Dose for swine, $10-25,3 \mathrm{ijss}$ to vj.

20. Manna. A mild cathartic. Dose for dogs, 10-50, 3 ijss to xij.

21. Glycerinum. Glycerin. Used in the form of clysters. An uncertain cathartic.

Patients, especially horses and cattle, should be prepared for the action of a cathartic; feed should be withdrawn and plenty of drinking water given. If there is no action within the usual time 
the animal should be given exercise at a walk. Usually, the dose should not be repeated. During catharsis the animal should be protected from cold and overexertion. These statements apply especially to aloes and croton oil. Generally, only one cathartic is given, as eserine, arecoline, calomel, aloes or castor oil. Sometimes several are given, one after another, when the first one fails to operate. In the Berlin medical clinic, for example, in cases of simple constipation-colic of the horse, arecoline is first administered. If, after an hour, no action occurs, then eserine is injected. If the eserine also fails to operate in an hour (intestinal obstruction), then an aloes pill is given. Two aloes pills, one shortly after the other, are dangerous and should not be given. In some cases several drugs are combined; for example, aloes with sodium sulphate, or croton oil with castor oil. Tartar emetic and aloes should not be administered at the same time (poisoning).

\section{CONSTIPATING REMEDIES. STYPTICS}

Synonyms: Enterostyptics, antidiarrhœecs, obstruents, antidysenterics, anticathartics, anexosmotics, astringents, protectives.

Actions and Uses.-The constipating drugs, which in their action are directly opposed to the cathartics, operate to check diarrhœa in several ways: by reducing increased sensibility of the intestinal mucous membrane; by depressing the increased peristalsis; by contracting the hyperæmic and swollen mucous membrane, and finally by inhibiting abnormal fermentation which may be the cause of intestinal irritation. These drugs are accordingly divided into the following groups:

(a) Demulcent Styptics (Protectives).-Linseed, althæa, acacia, compound acacia powder (N.F.), tragacanth, *salep, *mallow, emulsions, mucilaginous food materials (mucilage of oats and rye, rice water). The vegetable mucilage contained in these substances covers the inflamed and abnormally sensitive intestinal mucous membrane, preventing the intestinal contents from coming into direct contact with it and protecting it from irritation. The drugs in this group of styptics are only mildly constipating and are 
indicated only in slight diarrhœa or in the beginning of the condition. They are frequently combined with styptics of the other groups.

(b) Narcotic StYPtics.-Opium, morphine, and codeine. These anæsthetize the intestinal mucous membrane and thereby prevent the reflex stimulation of peristalsis. They also stimulate the splanchnic nerve, the inhibitory nerve of theintestines. Opium is the most valuable antidiarrhœic and should be prescribed as early as possible and in sufficient quantity in every severe case of diarrhœa, dysentery, and enteritis. It is frequently administered with mucilaginous drugs.

(c) Astringent Strytrcs.-Tannin or tannic acid, *tannoform, *tannalbin, *tannigen, *tannopin (the last three very expensive); the tannin-containing vegetable astringents: oak bark, cinchona bark, rhubarb, nutgall, *atechu, ${ }^{2}$ rhatany, *tormentilla root, salvia leaves, uva ursi, *folia juglans, red wine, *coffee and *roasted acorns; the metallic astringents: alum, iron sulphate, lead acetate, argentic nitrate, zinc oxide, argilla, and lime water. These drugs operate by constricting the blood vessels of the hyperæmic and inflamed intestinal mucous membrane, by contracting the latter and expressing the fluids, and by the formation of a scab of tannin or metallic albuminate. The metallic astringents are the most constipating, while the vegetable astringents are frequently ineffective in severe diarrhœa, especially in horses and cattle, even in very large doses.

(d) Antiseptic Stryptics.-Calomel, *creolin, tar, creosote, bismuth subnitrate and salicylic acid. These operate by suppressing or destroying the bacteria of fermentation and infectious materials in the intestinal canal which cause diarrhœa by their decomposition products. Dysentery and other infectious diarrhœas especially are effectively treated with creolin, calomel or salicylic acid. Even anthrax in cattle has been cured many times by creolin.

Drugs.-1. Opium. An excellent styptic for all domestic

2 [Replaced by gambir in U. S. Pharmacopœia, 1900.] 
animals in dysentery, intestinal catarrh and enteritis. Dose for horses, 5-20, $3 \mathrm{j}$ to v; cattle, 10-25, 3 ijss to vj; sheep, goats, foals, calves, and swine, $1-3$, grs. $x v$ to xlv; dogs, $0.1-0.5$, grs. jss to vij; cats, $0.05-0.2$, gr. $3 / 4$ to iij; chickens, 0.1 , grs. jss; pigeons, 0.05 , gr. $3 / 4$. Dose of Tinctura opii ten times these quantities. *Pantopon is an artificial mixture of the alkaloids of opium.

2. Acidum tannicum. Tannic acid or tannin. A styptic for the lighter diarrhœas of mycotic, toxic, and infectious origin. Dose for horses, $5-15$, $3 \mathrm{j}$ to $3 \mathrm{jv}$; cattle, 10-25, 3 ijss to vj; sheep and goats, 1-2, grs. xv to xxx; dogs, 0.1-0.5, grs. jss to vij; fowl, $0.01-0.05$, gr. $1 / 8$ to $3 / 4$. Dose of the tannin-containing vegetable drugs, Quercus (oak bark), etc., for horses and cattle, 25-50, 3vj to xij. The action of *tannalbin, *tannopin, *tannoform, and the other newer preparations is similar to that of tannic acid. The dose of tannoform for horses and cattle is 10-25, $3 \mathrm{ijss}$ to vj; calves and foals, $5-10,3 \mathrm{j}$ to ijss; dogs, 1-2, grs. $\mathrm{xv}$ to $\mathrm{xxx}$.

3. Rheum. Rhubarb. A valuable antidiarrhœic in dysentery of sucklings, especially white scours of calves and foals. Dose for horses and cattle, $25-50,3 \mathrm{vj}$ to xij; calves and foals, $5-10,3 \mathrm{j}$ to ijss; dogs, 1-2, grs. xv. to xxx; lambs, fowl and cats, $0.5-1$, grs. vij to xv. Of tinctura rhei, the dose for dogs is $1 / 4$ to 1 teaspoonful.

4. Alumen. Alum. A mild styptic in diarrhœa and intestinal hemorrhage. Dose for horses and cattle, 10-25, 3 ijss to vj; dogs, $0.5-2$, grs. vij to $\mathrm{xxx}$.

5. Ferri sulphas. Iron sulphate. A more powerful styptic, especially for infectious diarrhcas, dysentery, fowl cholera, and gastric and intestinal hemorrhages. Dose for horses, 10-25, 3 ijss to vj; dogs, $0.1-0.5$, grs. jss to vij; fowl, $0.05-0.2$, grs. $3 / 4$ to iij.

6. Liquor calcis. Lime water. A good styptic in diarrhca of sucklings, dysentery and intestinal ulcers. Dose for calves, $1 / 2$ to 1 pint; dogs, 1 to 2 tablespoonfuls. Calcii carbonas præcipitatus (precipitated calcium carbonate) has a similar action. [Dose for

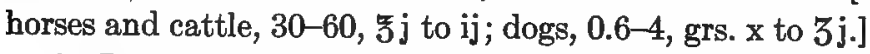

7. Plumbi acetas. Lead acetate. A very powerful styptic in persistent diarrhcea; must be administered to cattle cautiously. Dose for horses, 2-10, 3 ss to ijss; cattle, 1-5, grs. $x v$. to $3 j$; sheep, 
goats, and swine, 0.3-1, grs. jv to xv; dogs, $0.05-0.3$, grs. $3 / 4$ to jv; cats and fowl, $0.01-0.05$, grs. $1 / 8$ to $3 / 4$.

8. Argenti nitras. Silver nitrate. The most powerful styptic. Specific for intestinal ulcers. Dose for horses and cattle, 0.5-2, grs. vij to $\mathrm{xxx}$; sheep, goats and swine, 0.05-0.1, grs. $3 / 4$ to jss; dogs, $0.01-0.05$, grs. $1 / 8$ to $3 / 4$; fowl and cats, $0.01-0.02$, grs. $1 / 8$ to $1 / 4$.

9. ${ }^{*}$ Creolin. A specific against all diarrhœas of mycotic and infectious origin, calf cholera and diarrhœe of pigs. Dose for horses and cattle, 10-25, 3 ijss to vj; calves, sheep, goats, and swine, $0.5-1$, mpvij to xv; dogs, $0.5-1$, mpij to $\mathrm{xv}$; fowl, $0.1-0.25$, mojss to jv. Naphthalenum and resorcinol (resorcin) may be used in the same doses.

10. Hydrargyri chloridum mite. Calomel. A styptic in the dysenteric type of diarrhcea in the dog. Dose, 0.03-0.1, grs. $1 / 3$ to jss. Not to be administered to cattle, sheep or goats.

11. Bismuthi subnitras. Bismuth subnitrate. A disinfectant styptic for all of the domestic animals. Dose for horses, 5-15, $3 \mathrm{j}$ to jv; dogs, $0.25-1$, grs. jv to $\mathrm{xv}$.

12. Acidum salicylicum. Salicylic acid. An important disinfectant styptic in calf cholera. Dose, $1-5$, grs. $x v$ to $3 \mathrm{j}$ in alcoholic solution.

\section{MECHANICAL AND OPERATIVE TREATMENT}

The depressed peristalsis of constipation and colic may be stimulated reflexly by mechanical means. Massage of the abdomen, which is especially valuable in coprostasis in dogs; the application of irritants to the skin; exercise; the manual emptying of the rectum; infusions of water and rectal clysters (soap, oil, glycerin, suppositories), and massage of the intestines per rectum in colic in horses all operate in this manner.

Operative measuresinclude puncture of the intestines with trocar and cannula (flatulent colic), enterotomy (foreign bodies in dogs), laparotomy (invagination and incarceration of intestinal loops in cattle), and the reposition of the displaced colon in horses by rolling as in torsion of the uterus. The operative treatment of 
herniæ, invaginations and prolapse of the intestines is a chapter in itself.

The physical treatment of colic in horses is especially important. Rectal examination and treatment has always received great consideration in the clinics and from judicious practitioners. (See the text-books on special pathology and therapeutics.)

Carminatives. Gas-expelling Drugs.-The name carminative is used to designate a group of drugs which remove flatulence from the intestines. Abnormal fermentation of the intestinal contents produces collections of gases (carburetted hydrogen, hydrogen, hydrogen sulphide, carbonic acid) which cause flatulence and tympanites, with painful distention and tetanic contraction of the intestinal wall (flatulent colic). In horses the gaseous collection occurs especially in the large intestines; in cattle, in the rumen; in the small domestic animals, in the small and large intestines. Three varieties of carminatives are recognized, as follows:

(a) Antispasmodics: This group includes the ethereal oils-caraway, fennel, anise, chamomile, peppermint, melissa, valerian, angelica, garlic, and asafetida; also chloral hydrate, ether and spirit of ether. The aromatics (caraway, chamomile, peppermint, etc.) possess antispasmodic and antifermentative properties and are the most used carminatives. Dose for the horse, 10-25, 3 ijss to vj; cattle, $25-50,3$ vi to xij; dogs, $0.5-1$, grs. vij to xxx. Dose of the ethereal oils: horses, 1-5, परxv to $3 \mathrm{j}$; dogs, drop doses. Ether is a good carminative for horses and dogs. Dose for horses, 25-50, 3vj to xij; dogs, 2-5, 3 ss to $j$ (internally).

(b) Gas absorbents: Compound rhubarb powder, magnesium oxide, lime water, ammonia water. Pulvis thei compositus is an important carminative (absorbent) for sucklings.

(c) Anti-ferments: *Creolin, calomel and naphthalin. Creolin is given to horses and cattle in doses of 10-25, 3ijss to vj; calves, sheep and goats, $0.5-1$, mevij to $\mathrm{x}$.

\section{General Therapeutics of the Diseases of the Liver}

Pathology.-The therapeutic importance of diseases of the liver in animals is relatively slight compared to man. Of most frequent occurrence and of greatest practical importance are the parasitic diseases, especially distomatosis (liver rot) and echinococcus cysts, but these cannot be treated directly, and can at best only be influenced by prophylactic measures. This is also true of 
eirrhosis of the liver and acute yellow atrophy (lupinosis). Gallstones, abscesses, ruptures, and carcinoma are rare and as a rule are incurable. There remains for practical therapeutics only icterus, which is frequently presented for treatment, especially in dogs.

Physiology.-The physiological functions of the liver are not entirely understood. In addition to the secretion of bile (digestion of fat) and the production of glycogen (regulation of carbohydrate metabolism), the liver appears to be active in the removal of poisons from the body. This function is exercised chiefly in the excretion of toxic substances formed in the body (auto-intoxication), but poisons and drugs are also eliminated. In addition, antitoxic substances appear to be formed in the liver to protect and immunize the body against infectious diseases; an example of this is the immunizing action of the bile in rinderpest. Finally, important transformations and metabolic processes take place in the liver (disintegration of red blood-cells, formation of urea and uric acid, decomposition of alkaloids). On the other hand, recent investigations cast doubt upon the antiseptic properties formerly attributed to bile by which it was assumed to prevent putrefaction in the intestines (only the free bile acids exert a disinfectant action upon the intestinal contents).

Therapeutic Methods.-In icterus and in circulatory disturbances of the liver (acute and passive hyperæmia, hepatitis), the liver can be affected either by stimulation of the secreting liver cells, or by removal of mechanical obstructions to the outflow of bile, or by regulating the circulation of blood in the organ. Cathartics and intestinal disinfectants act as prophylactics by protecting the liver from harmful material in the intestines. The liver also in some diseases possesses a pronounced capacity for self-protection and for compensatory processes (compensatory hypertrophy in distomatosis).

The methods of treatment most frequently used are the medical (cholagogues), dietetic and mechanical. The operative methods used in human medicine for gall-stones, abscesses, wounds, and echinococci are of no practical importance in veterinary medicine. 


\section{CHOLAGOGUES. STIMULANTS TO BILE SECRETION}

Synonyms: Hepatics, hepatic stimulants, liver remedies.

Actions and Uses.-Cholagogues increase the secretion of bile by direct action upon the gland cells of the liver and in part by reflex stimulation from the stomach and intestines. They are used in all diseases of the liver in which the secretion of bile is decreased, as in icterus. According to the investigations of Rutherford (British Medical Journal, 1877), which have been recently amplified and confirmed by Ellenberger, Baum, Doyon, Dufour, Wissogradow and others, two classes of cholagogues must be recognized: (a) those which produce a laxative effect simultaneously with their action upon the liver and (b) those which operate exclusively upon the liver.

(a) The cholagogue laxatives are rhubarb, aloes, podophyllum, jalap, colocynth, gamboge, colchicum, arecoline, pilocarpine, eserine, euonymus, leptandra, baptisin, sodium sulphate, potassium sulphate, sodium phosphate, Carlsbad salts and potassium bitartras.

(b) The simple cholagogues are sodium bicarbonate, sodium salicylate, aspirin (acetyl-acidum salicylicum) and other salicylates, benzoate of soda, ipecac and nitric acid.

Calomel, epsom salts, castor oil and croton oil, which were formerly valued as cholagogues, are on the contrary depressants of bile secretion in consequence of their strong stimulant effect upon the intestinal glands. They are therefore designated as anticholagogues. Lead acetate also decreases the secretion of bile.

Drugs.-1. Rheum, aloe, sodii sulphas, sal Carolinum factitium N.F. (artificial Carlsbad salts), etc. For doses, see under Cathartics, pages 44, 45.

2. Ipecacuanha. Ipecac. Dose for swine and dogs, 1-3, grs. $\mathrm{xv}$ to $\mathrm{xlv}$.

3. Sodii salicylas. A very good cholagogue. Dose for horses and cattle, 25-75, 3 vj to 5 ijss; swine, 2-5, 3 ss to $j$; dogs, $0.25-2$, grs. jv to xxx. *Aspirin is given in the same doses.

4. Acidum nitricum. Given to dogs in drop doses, diluted. 
Concerning the cholagogue action of the emetics, see page 34 . Bile secretion is also increased by large quantities of water and by a meat diet. The bile acids also have a cholagogue action, which explains the former use of bile (fel bovis). According to recent investigations, Carlsbad water only renders the bile more fluid and does not increase the secretion of bile proper.

\section{DIETETIC AND MECHANICAL METHODS}

In dogs affected with icterus, as in man, the secretion of bile may be increased by a diet of meat and milk and by the administration of large quantities of water. The latter facilitates the reabsorption from the intestines into the blood of the bile salts, which have a cholagogue action (Schiff).

The circulation of blood in the liver is mechanically stimulated, directly or indirectly, by exercise, massage of the liver (applied in dogs under the right ribs), cutaneous irritation, cold infusions into the intestines, emetics and cathartics. 


\section{GENERAL THERAPEUTICS OF THE DISEASES OF THE ORGANS OF CIRCULATION}

\section{General Therapeutics of the Diseases of the Heart}

Pathology.-Therapeutically, the most important heart diseases of the domestic animals are chronic endocarditis (valvular insufficiency) and cardiac dilatation (heart weakness) of horses, cattle and dogs. On the other hand, the most frequent heart disease of cattle, traumatic pericarditis, due to the passage of metallic foreign bodies from the reticulum through the diaphragm to the pericardium and heart, is practically not affected by therapeutic measures. Chronic endocarditis leads to valvular insufficiency, with secondary compensatory hypertrophy, and later to cardiac dilatation. In the horse and dog there is usually an insufficiency of the mitral valve; in cattle, more frequently an insufficiency or stenosis of the tricuspid. Acute cardiac dilatation and cardiac degeneration occurs frequently in all the domestic animals, but especially in the horse and dog. It is manifested by cardiac weakness and is usually associated with infectious diseases: contagious pneumonia, foot-and-mouth disease, septicæmia; sometimes with overexertion (horses). Fatty degeneration of the heart is frequently observed in dogs and swine.

Physiology. - The action of the heart consists of a rhythmical contraction and relaxation of the cardiac muscle (systole, diastole, pause). During systole the blood is under positive pressure; during diastole it is under a negative pressure, corresponding to the sucking of a pump. The pause, or period of rest, is especially important because it affords opportunity for the recuperation of the heart. The valves serve to regulate the flow of blood. The cardiac muscle is innervated by the vagus, the inhibitory nerve, and by the sympathetic, which is the accelerator nerve; in addition, there are also automatic intracardial centres. The heart muscle and the nerves as well can be therapeutically stimulated by mechanical, chemical, thermic and electrical stimuli. On the other 56 
hand, paralysis of the heart can result from numerous causes, such as cardiac poisons, disease and overexertion of the myocardium, deficiency of oxygen, insufficient nourishment, and the collection of carbon dioxide and other products of decomposition. The cardiac muscle possesses in a high degree the capacity to accommodate itself to changed relations resulting from disease. An example is the compensatory hypertrophy of valvular insufficiency. The recognition of this compensation as a natural healing process and not something to be combated by therapeutic measures is of primary importance in the rational treatment of heart diseases.

Therapeutic Methods.-Disturbances of the pumping mechanism of the heart may result from disease of the muscular apparatus, the nervous system, the valvular mechanism or the nutritive blood-vessels. The treatment required will depend upon the condition present. Drugs affecting the muscles or the nerves, mechanical methods or vasodilators may be indicated. The complicatedmechanical, thermic and electrical methods of human medicine (change of climate, baths, hydrotherapy, electrotherapy, inhalation of compressed air) are not applicable in veterinary medicine. In the latter, treatment of cardiac diseases is limited mainly to the use of medicines. The rest treatment can be applied to animals as to man; in cardiac weakness the heart can be protected by reducing or abolishing work and exercise. In certain conditions, as obesity in dogs, systematic exercise and dietetic treatment may be instituted. Regarding the latter, decreasing or regulating the drinking water, thereby reducing the amount of fluid in the blood and decreasing the work of the heart, is of practical importance (Oertel's method). In dogs, as in man, food that is too bulky can be avoided (milk diet). The operative treatment of cardiac diseases, as puncture of the pericardium in pericarditis, is hardly worth considering.

\section{CARDIACS. HEART REMEDIES}

Synonyms: Polysphygmics, bradysphygmics, heart stimulants, heart tonics, heart sedatives.

Classification.-The cardiacs are classified according to their action. There are three groups: stimulants, tonics and sedatives. 
1. The cardiac stimulants are camphor, hyoscine (scopolamine), atropine, caffeine, veratrine, alcohol, ether, ammonia, ammonium carbonate and some of the ethereal oils (valerian, arnica). These act in part by paralyzing the vagus, the nerve of inhibition, centrically or peripherally; in part by stimulating the sympathetic nerve and the vasomotor centre, and in part by stimulation of the heart muscle.

2. The cardiac tonics are digitalis, strophanthus, squill, sparteine, adonidin and convallamarin. These stimulate the vagus centrically and peripherally, and also the heart muscle.

3. The cardiac sedatives are potassium bromide, chloral hydrate and potassium iodide. These act in part by depressing the cardiac centre in the medulla oblongata and in part by depressing the cardiac muscle.

Uses.-1. The cardiac stimulants are prescribed in all cases of acute heart weakness such as may occur in the course of acute heart diseases, febrile infectious diseases and poisoning; also in syncope. The most important symptoms of heart weakness are a weak or imperceptible, very frequent or slow pulse; in the beginning a throbbing heart beat, which is later weak or imperceptible; indistinct heart sounds, dyspnœea, cyanosis of the mucous membranes, collapse.

2. The cardiac tonics are specific for chronic heart diseases, particularly valvular defects in the stage of insufficiency. They increase the force of contraction of the heart muscle, slow and regulate the rate of contraction, increase the length of the pause between beats, and increase the blood-pressure. They are indicated when the pulse is abnormally rapid, irregular and weak, and the heart is beating tumultuously. On the other hand, they are contraindicated in valvular insufficiency when compensatory hypertrophy has occurred, in cardiac hypertrophy, in palpitation and in all cases in which the pulse is strong or the heart beat abnormally slow (bradycardia).

3. The cardiac sedatives are prescribed in cardiac excitement of nervous origin, particularly palpitation.

Drugs.-1. Digitalis. The most important cardiac for val- 
vular insufficiency without compensation. Dose of the leaves for horses and cattle, 2-5, 3 ss to $j$; dogs, $0.05-0.2$, grs. $3 / 4$ to iij. Dose of the leaves as an antipyretic for horses, $5-10,3 \mathrm{j}$ to ijss.

2. Camphora. A powerful stimulant for all kinds of heart weakness. Used subcutaneously in the form of spiritus camphoræ or dissolved in olive oil $(1: 9$ or $1: 4)$. The stronger camphorated oil $(1: 4)$ is indicated in contagious pneumonia of horses especially; it is injected subcutaneously in single doses of $50-150$, 5 jss to $\mathrm{v}$; daily doses of $100-250$, 3 iij to viij. The doses heretofore were too small.

3. Caffeina. Caffeine. One of the best cardiacs in acute heart weakness; acts very quickly when given subcutaneously; specific antidote against heart poisons. Dose of *caffeinæ sodiosalicylas (N.F.) for horses and cattle, $5-10,3 j$ to ijss; large dogs, $0.5-2$, grs. vij to $\mathrm{xxx}$; small dogs, 0.1 to 0.5 , grs. jss to vij. Also administered in the form of coffee.

4. Tinctura strophanthi. A substitute for digitalis. Dose for horses, 4-15, 3j to jv; dogs, 2 to 16 drops. Strophanthin, the active principle, produces necrosis when injected subcutaneously.

5. Atropinæ sulphas and hyoscinæ hydrobromidum (scopolamine). Very strong stimulants in heart weakness in the course of heart diseases, infectious diseases and poisoning. Act very quickly when injected subcutaneously. Dose of atropine sulphate for horses and cattle, $0.05-0.1$, grs. $3 / 4$ to jss; dogs, $0.01-0.05$, gr. $1 / 8$ to $3 / 4$. Dose of hyoscine (scopolamine) hydrobromide onetenth that of atropine sulphate.

6. Veratrina. Veratrin. A powerful stimulant in the heart weakness of fever. Dose for horses and cattle, $0.05-0.2$, grs. $3 / 4$ to iij, subcutaneously in solution in alcohol; dogs, 0.001-0.005, gr. $1 / 70$ to $3 / 40$.

7. Alcohol. An excellent stimulant in all weak conditions of the heart. Dose for horses and cattle, $25-50,3 \mathrm{vj}$ to xij; sheep and goats, $10-20,3$ ijss to jv; dogs, 2-5, 3ss to j. Dose of spiritus vini gallici (brandy) for horses and cattle, 50-150, 甬jss to $5 \mathrm{v}$; dogs, tablespoonful.

8. Ether. The same. Dose for horses and cattle, subcutaneously, 10-25, 3 vj to xij; dogs, $0.5-2$, mvij to $x x x$. 
9. Ammonii carbonas. Ammonium carbonate. A good and cheap stimulant in heart weakness. Dose for cattle, $20-50,3 \mathrm{jv}$ to xij; horses, 10-25, 3 ijss to vj; sheep, goats and swine, 1-2, grs. $\mathrm{xv}$ to $\mathrm{xxx}$.

10. Valeriana. Valerian. An old but good stimulant; used in the form of tinctura valerianæ. Dose for horses and cattle, 20-50, $3 \mathrm{jv}$ to xij; sheep and goats, $2-5,3$ ss to $3 \mathrm{j}$; dogs, 0.2-2, miij to $\mathrm{xxx}$; cats and fowl, $0.1-0.5$, mijss to vij.

11. Potassii iodidum. Potassium iodide quiets the heart and reduces blood-pressure in hypertrophy and palpitation. Dose for horses and cattle, $5-15,3 \mathrm{j}$ to jv; dogs, $0.25-1$, grs. iij to xv; cats, $0.1-0.2$, gr. jss to iij.

12. Potassii bromidum and chloralum hydratum. Sedatives in palpitation of the heart. Dose for horses and cattle, $20-50,3 \mathrm{jv}$ to xij; dogs, 1-2, grs. xv to $\mathrm{xxx}$.

\section{General Therapedtics of the Diseases of the Blood}

Pathology. - The diseases of the blood consist in a defect in the formation of the blood. The total quantity may be decreased (anæmia, pernicious anæmia) or, as more rarely happens, increased (plethora); the number of white blood-cells may be greatly increased (leukæmia), the number of red cells may be considerably decreased (oligocythæmia), or the water content of the blood may be increased (hydræmia). Pernicious anæmia is a specially severe form of anæmia with characteristic morphological changes in the red bloodcells (poikilocytosis, macrocytosis, microcytosis). In addition to these primary diseases of the blood, anæmia and hydræmia very frequently occur secondarily in chronic disturbances of nutrition and in infectious diseases (chronic gastro-intestinal catarrh, tuberculosis, carcinoma cachexia, distomatosis) and after loss of blood. An abnormal thickening of the blood in consequence of the elimination of water occurs in heat stroke and in profuse diarrhœa. Finally, the blood shows severe parenchymatous and chemical changes in fever (see the chapter on fever) and after the operation of poisons such as potassium chlorate, paraldehyde, snake venom, carbon monoxide, saponin, ricin and other ferments; also in infectious diseases. 
Physiology.-The blood transports the oxygen and the fluid and solid nutrients (albumin, fats, carbohydrates, salts, and water) to the tissues and organs and carries away the decomposition products of metabolism, especially the carbon dioxide (lungs) and the fluid and solid excretions (kidneys, liver, intestinal glands, cutaneous glands). The most important morphological elements of the blood are the red and white corpuscles (the blood-platelets are regarded by some as products of the decomposition of the white blood-cells, by others as hæmoglobin-free bodies from the interior of the red blood-cells). The red blood-cells (erythrocytes without nuclei) are derived from the red bone marrow. They are, on account of the hæmoglobin they contain, the carriers of oxygen and form about one-third of the volume of the blood. A cubic millimetre of horse's blood contains seven to eight million red blood-cells. The white blood-cells (leucocytes) originate in part from the red marrow of bone (granular leucocytes) and in part from the lymph glands and spleen (lymphocytes without granulation). They are much less numerous than the red blood-cells, there being only one white corpuscle to 350 red; one cubic millimetre of horse's blood contains 9,000 leucocytes. Their functions are manifold: fat resorption in the intestines, emigration, phagocytosis, immunization in inflammatory and infectious diseases and histogenetic activity in healing of wounds and in cicatrization.

The reaction of the blood is alkaline $\left(\mathrm{KHCO}_{3}\right)$, corresponding to a 0.2 to 0.4 per cent. soda solution. The most important chemical constituents are the proteids: hæmoglobin, serum albumin, serum globulin, nucleo-albumin, albumoses, peptone, lecithin and protagon; the fats: stearin, palmatin and olein; the carbohydrates: grape sugar and glycogen; the pigments: hæmoglobin and bilirubin; and the salts: sodium, potassium, calcium, magnesia, iron and ammonium combined with chlorine, carbonic acid, phosphoric acid and sulphuric acid. The potassium salts are contained in the blood-cells, the sodium salts in the blood-serum. In herbivora the carbonates, in carnivora the phosphates, predominate.

The quantity of the blood is equal to about one-thirteenth of the body weight; the relative amount is greatest in the horse (one- 
tenth) and least in swine (one-twentieth). The distribution of the blood is of importance in therapeutics. While the body is at rest one-fourth of the blood is contained in each of the following sections: the heart and large blood-vessels; the liver; the muscles; the other organs; but during work the muscles contain up to twothirds of the total quantity.

Therapeutic Methods.-The quantity, composition and distribution of the blood in disease can be affected in very different ways. The dietetic method supplies to the diseased blood bloodforming substances (blood plastics) of all kinds (proteids, iron, salts). The chemical method combats infectious agents contained in the blood by the introduction of arsenical preparations into the blood stream (chemotherapy); serum therapy acts in the same manner. The mechanical method seeks to influence blood formation indirectly by systematic stimulation of the muscles (muscle therapy, training, massage). The operative method consists in the introduction of fresh, healthy blood (transfusion) and the removal of diseased blood (phlebotomy).

Transfusion, i.e., the direct or indirect transfer of blood from a healthy individual to the blood stream of a diseased individual, appears theoretically to be very rational. In practice, however, it has not proven satisfactory. The transfusion of non-defibrinated blood is dangerous to life (fibrin emboli), and the transfusion of defibrinated blood has at times been attended with serious disturbances (rigors, albuminuria, anaphylaxis). The effects of the transfusion of animal blood (lamb's blood) into man consist of hyperæmia and serous infiltration of the internal parts of the body, high transfusion fever and a change in the composition of the blood. Experience in human medicine has also taught that the danger from excessive bleeding is not so much from the decrease of red blood-cells as from the volume of the blood being insufficient to fill the heart and vessels, and for this reason the injection of a simple physiological salt solution, subcutaneously or intravenously, will save life in such cases.

In regard to the effect of phlebotomy on the blood, practical experience and scientific investigation are not in accord. Small, 
daily bleedings are supposed to stimulate blood formation and increase the red blood-cells in chlorosis of man. Some have reported unfavorable results from phlebotomy in anæmia. The only undisputed indication for phlebotomy is plethora.

DIETETIC METHOD. BLOOD-FORMING REMEDIES. BLOOD PLASTICS

Synonyms: Hæmatics, hæmatopoietica, hæmatinics, erythrotics, hyperinotics, euplastics.

Actions.-Blood-forming agents may act directly or indirectly.

1. The direct blood plastics are substances which are normal constituents of the blood: albumin, peptone, iron, potassium salts, sodium chloride, phosphates, sulphur, meat extract, fat. They supply directly to the blood the constituents which are absent in disease.

2. The indirect blood plastics are not normal constituents of the blood, but improve the condition of the blood by increasing metabolism, assimilation and nutrition. The most important indirect blood plastic is arsenic. Others are alcohol, phosphorus, and mercury in very small doses, and the alkalies.

In practice, it is important that the blood plastics be administered in a form which can be readily absorbed and in small, repeated doses. The iron salts and arsenic especially are to be given with great care as to the dosage.

Drugs.-1. Ferrum. Iron. The principal remedy in anæmia, hydræmia and leukæmia. The preparations most commonly used in veterinary medicine are: ferri reductum (reduced iron), ferri sulphas, *tinctura ferri pomata (N.F.). The numerous newer and more costly iron preparations, and also the new albumin preparations, are not necessary in veterinary medicine. Dose of reduced iron and iron sulphate for horses and cattle, 1-5, grs. $\mathrm{xv}$ to $3 \mathrm{j}$; dogs, $0.02-0.2$, grs. $1 / 4$ to iij; sheep, $0.5-1$, grs. vij to xv. Dose of tincture ferri pomata for dogs, 10 to 20 drops. Concerning the absorption of iron preparations, see text-books on pharmacology.

2. *Meat extract. This acts as a hæmatopoietic by supplying blood salts, potassium salts and phosphates. Dose for dog, 2-10, $\boldsymbol{Z}$ ss to $\bar{Z}$ ijss. 
3. Potassii bicarbonas. Potassium bicarbonate is used as a blood plastic in anæmia. Dose for horses and cattle, 25-100, $3 \mathrm{vj}$ to 年ij; sheep, $2-5,3$ ss to $\mathrm{j}$; dogs, $0.5-2$, grs. vij to $\mathrm{xxx}$.

4. Sodii chloridum and sodii phosphas. Sodium chloride and sodium phosphate are used as blood plastics in chronic disturbances of nutrition. Doses same as potassium bicarbonate.

5. Sulphur sublimatum, sulphur præcipitatum, sulphur lotum. Sulphur is used as a blood plastic in specific blood diseases. Dose for horses and cattle, $2-5,3$ ss to $3 \mathrm{j}$; sheep, $0.5-1$, grs. vij to $\mathrm{xv}$; dogs, $0.05-0.2$, grs. $3 / 4$ to iij.

6. Arseni trioxidum. Arsenic. A valuable blood plastic in all blood diseases and chronic disturbances of nutrition. Dose for horses and cattle, 0.1-0.5, grs. jss to vij; sheep, 0.005-0.01, gr. $3 / 40$ to $1 / 8$; dogs, $0.001-0.005$, gr. $1 / 70$ to $3 / 40$. The dose of liquor potassii arsenitis (Fowler's solution) for horses and cattle is $10-50$, 3 ijss to xij; sheep, $0.2-0.5$, miij to vij; dogs, $0.1-0.2$, mijss to iij.

\section{General Therapeutics of Diseases of the Blood- Vessels}

Pathology.-The diseases of the blood-vessels which call for therapeutic interference are wounds and tears or ruptures (arterial, venous and capillary hemorrhage), circulatory disturbances (anæmia, active and passive hyperæmia, thrombi, emboli), inflammation (arteritis, phlebitis), dilatations (varices, aneurisms) and neoplasms (angiomas, endotheliomas). The pathological changes may involve the vessel walls, the vasomotor nervous system or the vessel contents, i.e., the blood (quantity, distribution, property, coagulation, blood-pressure, circulation).

Physiology.-Of the three layers of the blood-vessel wall the most important is the middle one, the muscular layer, which is the motor organ for the constriction of the vessel. This layer is absent from the capillaries, the walls of which consist of only contractile endothelial cells. The nerves of the vessels (vasomotors) are situated centrally as well as peripherally. The principal vessel centre (vasomotor centre) is located in the medulla oblongata; 
there are additional centres in the spinal cord. The peripheral nerves which are connected with these centres possess in part the function of contracting (vasoconstrictors, pressors) and in part of dilating (vasodilators, depressors) the blood-vessels. The peripheral vessels also appear to possess independent vasomotor ganglion cells.

The vasomotor nervous apparatus can be stimulated or depressed by agents acting centrically or peripherally. The direct centric stimulants (vasoconstrictors) are digitalis, strychnine, nicotine and carbon dioxide; insufficient oxygen in the blood has the same effect. The depressants or vasodilators are alcohol, ether, chloroform and amyl nitrite. Contraction and dilation of the vessels are also produced directly or indirectly (reflexly) by different agents acting peripherally (mechanical, chemical, thermic, and electrical stimuli applied to the skin or mucous membranes). Adrenalin is believed by some to cause vasoconstriction by centric stimulation, others attribute its action to a peripheral stimulant effect upon the vessels.

Contraction of the vessels increases while dilatation decreases the blood-pressure. The normal blood-pressure in the carotid artery is equivalent to 150 millimetres of mercury; in the aorta to 200 to 250 millimetres. The greatest increase in blood-pressure is produced by increase of the heart action and contraction of the bloodvessels simultaneously (digitalis, strophanthus). An increase in blood-pressure also occurs when areas supplied by the larger vessels are shut off from the circulation and when there is any interference with the circulation of the blood in the larger arteries (insufficiency of the aortic valves, thrombosis of the pelvic or femoral arteries). In the latter conditions the continued high blood-pressure results in a compensatory hypertrophy of the heart.

The rate of flow of the blood in the carotid artery of the horse is, on the average, 300 millimetres. It is dependent upon the activity of the heart, the contraction of the blood-vessels and the depth of respiration (aspiratory effect of deep inspiration).

The causes of the coagulation of the blood have not been satisfactorily explained. The fibrin which is in solution in the blood 
may be precipitated as a calcium-albumin compound through the coöperation of three factors: 1. Fibrin ferment (a product of the decomposition of white blood-cells). 2. The fibrinogens. 3. The fibrinoplastic substances: fibrinogen and paraglobulin.

Therapeutic Methods.-Wounds and the consequent hemorrhage are the conditions which most frequently affect the bloodvessels, and for this reason the method of arresting hemorrhage will first be considered. Other therapeutic methods are concerned with the contraction and dilation of the blood-vessels (see also the antiphlogistic and resorbent methods).

\section{METHODS OF ARRESTING HEMORRHAGE}

Spontaneous Arrest of Hemorrhage.-By this is understood the cessation of bleeding as a result of a natural process, in contradistinction to the artificial arrest of hemorrhage by therapeutic measures. It is especially observed in capillary and parenchymatous hemorrhage, and also occurs when small arteries and veins are wounded. It is due principally to the coagulation of the blood, but retraction of the vessel wall and narrowing of the lumen of the vessel are also important factors. The coagulation of the blood forms a thrombus, which closes the opening in the vessel and sometimes extends into the interior. As the blood-pressure is very low in the capillaries and small veins, the formation of thrombi and the resulting arrest of hemorrhage can occur very quickly. But in large vessels, especially arteries, thrombi are not formed at all or only when the heart has become weak and the blood-pressure has been considerably lowered by severe hemorrhage, so that the coagulated blood is not washed away by the outpouring blood. In severe hemorrhage the blood, in an effort to rehabilitate itself, takes up numerous white blood-corpuscles, which increase its coagulability and thus assist in the spontaneous arrest of bleeding. This is the explanation of the fact, established experimentally, that stallions castrated without any precautions against bleeding do not die from loss of blood. Generally, however, not more than onethird of the total quantity of blood can be lost without causing fatal cardiac and cerebral paralysis. The blood regenerates itself 
quantitatively rather rapidly by the resorption of the lymph contained in the tissues and the fluids present in the stomach and intestines. The new-formed blood is at first very rich in water, while the red blood-corpuscles are few in number. The latter are replaced slowly.

The exact nature of the processes concerned in the formation of the thrombus is not entirely understood. In the spontaneous arrest of hemorrhage a white thrombus is generally present, formed by the aggregation of white blood-corpuscles and blood-platelets, and differing essentially from the usual products of coagulation, which consist of fibrin inside or outside of the body. The subsequent history of the white thrombus varies, depending upon whether infectious materials gain entrance to it or not. If it remains free from infection, organization takes place; i.e., a firm connective tissue develops, which is supplied with nutrient vessels, and which closes the wounded vessel with a solid and permanent cicatrix. The cells of the thrombus do not actively participate in the process; they play only a passive rôle, being gradually repressed by the newformed tissue. The formation of new connective tissue results from the growth of the vessel endothelium. The endothelial cells of the intima proliferate and are transformed into spindle-shaped and variously formed cells which extend toward the centre of the thrombus, grow into it and around it, and later are changed into fibrillar connective-tissue cells, the thrombus being thus finally compressed and replaced by firm connective tissue. At the same time, as a result of budding of the vasa vasorum, new vessels are formed in the thrombus and it becomes vascularized. About four weeks after the injury, in the smaller vessels, the thrombus is transformed into a mass of cicatricial connective tissue permeated with capillaries, which subsequently becomes more solid in consequence of contraction and the atrophy of the newly formed blood-vessels. More rarely, calcification of the thrombus occurs (so-called vein stones or phleboliths). The interruption to the circulation resulting from the thrombus is overcome by the establishment of a collateral circulation while the arterial branches situated centrally and peripherally to the thrombus and their vasa vasorum are dilated. 
Sometimes a passage for the blood is later opened through the centre of the organized thrombus, or the blood-vessels of the cicatrix become dilated and permit the blood to flow through.

Artificial Arrest of Hemorrhage.-This aims to close the bleeding blood-vessel either by pressure or by aiding coagulation. The most important methods used for this purpose are:

(a) Ligation of Blemeing Vessems.-The ligature is the only thing which will quickly and surely stop bleeding from the larger arteries and veins. The bleeding vessel is grasped with clamp forceps and tied with silk thread. If the vessel cannot be isolated, then the tissue surrounding it is included in the ligature (ligation en masse), a needle being used to carry the thread around through the tissues. When neither of these methods is applicable because of the deep situation of the bleeding vessel, then the ligature must be placed at some accessible point situated centripetal to the point of hemorrhage (ligation by continuity); e.g., in hemorrhage from one of the arteries in the interior of the head the carotid is ligated.

(b) Compression of the Vissels by Suture or Bandage, as a rule, checks parenchymatous and capillary hemorrhage. Sometimes strong pressure upon the bleeding vessels can be obtained by tamponading a wound. Bleeding may be checked temporarily by pressure with the fingers or hand (digital compression), or by applying an elastic tubing (Esmarch bandage) or a rubber bandage (Martin's bandage) between the wound and the heart. Methods of arresting hemorrhage peculiar to veterinary medicine are the use of clamps and of the emasculator in castration. The formerly employed tourniquet (a compress fastened with a girdle) and the so-called acupressure (compression by a needle inserted in the tissues in a transverse direction, laterally and above or below the bleeding vessel) are now scarcely used.

(c) Torsion of the Bleeding Vesser loosens the intima and media, which roll inward, and also draws the adventitia together, narrowing or closing the lumen of the vessel. Torsion is carried out in two ways: the bleeding vessel is grasped with the clamp forceps and revolved on its axis for some time, or the surrounding soft 
parts, including the vessel, are twisted (torsion of the spermatic cord).

(d) Hrat in the form of the hot iron or the thermo-cautery is an effective hæmostatic in many cases of parenchymatous hemorrhage. The opening in the bleeding vessel is closed by a scab, which acts as an aseptic bandage (cautery in amputation of the tail). CoLd (ice, cold water, ether spray) is less reliable; it checks hemorrhage by contracting and narrowing the small blood-vessels. Hot water at a temperature of 45 to $50^{\circ} \mathrm{C}$. is recommended as a good hæmostatic for parenchymatous bleeding. Even live steam, 100 to $120^{\circ} \mathrm{C}$. (?), has been directed into the uterine cavity for several minutes in persistent hemorrhage from the uterus in woman (so-called vaporization of the uterus, atmocausis, zestocausis).

(e) Drugs (hæmostatics, styptics, antihemorrhagics, astringents, agglutinatives, rophetics) are used in external parenchymatous and in internal hemorrhages. They are ineffective in external arterial and venous hemorrhages; in these cases surgical methods (ligature, compression) are of greater service. The mode of action of the styptics differs with the different drugs. Some cause a contraction of blood-vessels, with narrowing of the lumen, by direct action upon the muscular layer of the blood-vessel wall. In this way, for example, nitrate of silver reduces the lumen onehalf. The contraction of the vessels reduces the amount of blood, slows the circulation and finally causes stasis. Other drugs arrest hemorrhage by causing coagulation of the blood, directly or indirectly, and closing the lumen of the bleeding vessel by the resulting thrombus. The solution of chloride of iron acts in this way. Some drugs operate in both ways; e.g., tannic acid and ergot. Blood coagulation is also favored by decrease of blood-pressure. Some drugs act in a purely mechanical way by agglutinating the bleeding surface (agglutinative styptics), or by absorbing the blood (rophetic or spongy styptics, e.g., sponge). For practical purposes styptics are divided into two groups: local and general.

1. Local or topical styptics serve to arrest hemorrhage upon the skin, accessible mucous membranes and, when administered by inhalation, in the lungs. The most important are: solution of 
ferric chloride, tannic acid, nitrate of silver, chloride of zinc, lead acetate, alum, antipyrine, adrenalin (suprarenin), creolin, creosote, oil of turpentine, ether and acetic acid. To this group also belong the agglutinative styptics: acacia, tragacanth, colophony, collodion, starch, chalk and plaster of Paris; also the rophetic styptics: sponges, absorbent cotton and pengamar djambi (long, silky hairs from East Indian ferns, official in Austria as paleæ hæmostatica) which were formerly much used but which have become obsolete since the introduction of asepsis.

2. General styptics operate internally after absorption into the blood. The most important are ergot, hydrastis, lead acetate, tannic acid and alum. Digitalis, adrenalin (suprarenin), oil of turpentine and other ethereal oils, acids and sodium chloride also operate internally as hæmostatics. Subcutaneous injections of white gelatin, which is recommended as a general styptic, should be used with caution (tetanic convulsions in man). The internal styptics are employed in hemorrhages from the uterus, kidneys, bladder, intestines, stomach, liver, spleen, lungs and brain. In gastric and intestinal hemorrhages the action of most of these is, strictly speaking, a local one (tannic acid, lead acetate, alum, sulphate of iron, solution of ferric chloride). In general, the action of the internal styptics is very uncertain, except the effect of ergot and hydrastis upon hemorrhage from the uterus.

Drugs.-1. Ergota. Ergot. Specific against uterine hemorrhage. [Dose of fluidextractum ergotæ for horses and cattle, 15-30, 5 ss to $5 \mathrm{j}$; sheep, goats and swine, 4-8, $3 \mathrm{j}$ to $\mathrm{ij}$; dogs, 2-4, 3 ss to $\mathrm{j}$.]

2. Hydrastis. A specific against hemorrhages from the uterus and abdominal organs. [Dose of fluidextractum hydrastis for horses and cattle, 8-30, $3 \mathrm{ij}$ to $\mathrm{z}$; sheep and swine, 4-8, $3 \mathrm{i}$ to $\mathrm{ij}$; dogs, $0.3-4$, nve to 3 i.]

3. Acidum tannicum. Tannic acid or tannin. A styptic in gastric, intestinal and renal hemorrhage; external hæmostatic. Dose for horses and cattle, 5-25, $3 \mathrm{i}$ to vj; sheep and goats, 1-2, grs. $\mathrm{xv}$ to $\mathrm{xxx}$; dogs, $0.1-0.5$, grs. iss to vij. Quercus, cinchona, krameria, *tormentilla, uva ursi, *catechu and galla have the same action. 
4. Plumbi acetas. Lead acetate. A good hæmostatic in gastric hemorrhage. Used empirically in hemorrhage from the lungs, kidneys, bladder and uterus. Dose for horses, $2-10,3$ ss to ijss; cattle, $1-5$, grs. $x v$ to $3 \mathrm{i}$; dogs, $0.05-0.2$, grs. $3 / 4$ to iij.

5. Alumen. Alum. A styptic in intestinal hemorrhage. Dose for horses and cattle, 10-25, 3 ijss to vj; dogs, 0.5-2, grs. vij to $\mathrm{xxx}$.

6. Liquor ferri chloridi. Solution of chloride of iron. External styptic, undiluted, or with collodion $1: 10$, upon wounds; 1 to 5 per cent. solution in water on mucous membranes; in $1 / 2$ per cent. solution by inhalation in hemorrhage from the lungs. Internally, in gastric hemorrhage, freely diluted.

7. *Creolin. External styptic in operations in 3 per cent. solution.

\section{VASOMOTOR STIMULANTS. DRUGS THAT CONTRACT BLOOD-VESSELS}

Synonyms: Angiosthenics, vaso-astringents, vaso-constringents.

Actions.-A contraction of the blood-vessels (arteries, capillaries, veins) can be produced by influences acting either upon the nerves, the musculature of the vessels, or the blood. The various organs concerned are the vasomotor centre in the medulla oblongata, similar centres in the spinal cord, and the peripheral vasoconstrictor nerves; all of which can be stimulated directly and reflexly. Contraction of the blood-vessels causes a decrease in the amount of blood in the tissues they supply and an increase in the blood-pressure. Other vasoconstrictor drugs act by coagulating the blood or by withdrawing fluid. Vasomotor constrictors are divided into two groups: external and internal. The most important external vasoconstrictors are silver nitrate, lead acetate, copper sulphate, zinc sulphate, zinc chloride, bichloride of mercury, iron sulphate, alum, tannic acid, adrenalin (suprarenin), chalk and also mild cutaneous irritants. The most important internal vasoconstrictors are ergot, hydrastis, digitalis, caffeine strophanthus [atropine, strychnine and cocaine].

Uses.-The vasomotor stimulants are used to arrest hemorrhage (see previous chapter); to overcome hyperæmia, stasis and 
other circulatory disturbances in external and internal organs; to combat swellings, exudations, transudations, catarrhs, polyuria, ptyalism and hyperhidrosis; in the treatment of aneurisms, varicose veins and angiomas; to increase blood-pressure, and in the treatment of cardiac weakness. (See also chapters on astringents and antiphlogistics.)

Drugs.-1. Argenti nitras. Silver nitrate. The strongest astringent in inflammation of the skin and mucous membranes; best remedy in ulcers and burns; used in 5 to 10 per cent. solution in water or alcohol; as a salve, $1: 10$; in substance in pencil form; also in $1 / 2$ to 2 per cent. solution as an eye water.

2. Zinci sulphas. Zinc sulphate. Important astringent in the treatment of eye diseases; used in $1 / 2$ to 2 per cent. solution in water (conjunctivitis). On other mucous membranes it is used in 1 to 5 per cent. solution. In inflammatory and eczematous conditions of the skin, zinc sulphate and zinc oxide are used in the form of the powder and ointment.

3. Liquor plumbi subacetatis dilutus. Lead water. Goulard's water. Frequently used as an astringent in inflammation of the skin, burns, eczema and catarrhs of mucous membranes.

4. Cupri sulphas. Copper sulphate. Astringent in diseases of the eye and in foot rot.

*Adrenalin.-A constituent of the suprarenals which has vasoconstrictor properties and which acts locally in a dilution of one to ten million. Used locally; a few drops of a solution of adrenalin chloride in physiological salt solution (1:1000).

For other vasomotor stimulants see the previous chapter on hæmostatics; also chapters on astringents and antiphlogistics.

\section{VASODILATORS. AGENTS THAT DILATE BLOOD-VESSELS}

Synonyms: Angio-asthenics, vasodilatants.

Actions and Uses.-The vasodilators produce a dilation of the blood-vessels by causing a relaxation of the blood-vessel wall through depressing or paralyzing the vasoconstrictors or stimulating the vasodilators. They bring about an increased supply of blood, decrease of blood-pressure, slowing of the circulation, and, after prolonged action, stasis of blood with transudation of serum. 
The most important vasodilators are amyl nitrite, sodium nitrite, nitroglycerin, morphine, chloroform, chloral hydrate, potassium bromide, alcohol, ether, some of the ethereal oils; also heat and powerful cutaneous irritants.

The practical use of the vasodilators is very limited in veterinary medicine. They are prescribed, for instance, in cerebral anæmia (syncope), and also in some brain diseases which are attributed to a circumscribed vascular spasm in the brain, as in eclampsia and epilepsy. They are recommended in nervous asthma of the dog (nitroglycerin). In human medicine, the vasodilators are also prescribed in spasm of the coronary arteries of the heart designated as angina pectoris.

Drugs.-1. Amylis nitris. Amyl nitrite. May be given experimentally in eclampsia of suckling bitches; also by inhalation in asthma. [Dose for dogs, $0.03-0.2 ; \pi 1 / 3$ to iij.]

2. Nitroglycerin. The same. [Dose of spiritus glycerylis nitratis, formerly spiritus glonoini, for dogs, $0.06-0.13$, mj to $\mathrm{ij}$.]

3. Sodii nitris. The same. Dose for dogs, $0.1-1$, grs. iss to xv: For other drugs, see the chapter on sedative neurotics.

\section{General Therapedtics of the Exudates and Transu- DATES. Resorbents}

Pathology.-The diseased conditions in which the resorbents are indicated are solid and fluid inflammatory exudates, blood extravasations, transudates, œdemas in the body cavities and tissues, and inflammatory swellings and thickenings of the skin, subcutis, tendons, tendon sheaths, muscle, periosteum, bones and lymph glands. Hyperplasia of the thyroids (goitre) and of the lymph glands, actinomycosis, sediments, poisons and obesity are also treated with resorbents. On the other hand, true neoplasms, as carcinomas, sarcomas, lipomas and fibromas, cannot be removed with resorbents.

Physiology. - The removaı of collections of pathological fluids and of other pathological products from the organs and tissues results from the operation of the physiological processes concerned in the normal resorption of lymph and parenchymatous fluids. 
These complicated processes are dependent upon the laws of filtration, diffusion, capillarity, imbibition and phagocytosis. Primarily concerned in the processes are the blood and lymph vessels and the white blood-corpuscles, while normal resorption is influenced secondarily by cardiac activity (bloodpressure), respiration (blood aspiration) and exercise (muscular contraction).

The physiological activity of the blood and lymph vessels in resorption takes place in the capillaries and at the origin of the lymph vessels (interstitial and perivascular lymph spaces and canals, lymph stomata). The dissolved constituents of the lymph and parenchymatous fluids are absorbed into the blood and lymph channels according to physical laws. Active blood circulation, increased blood-pressure, accelerated cardiac action and a decrease in the quantity of the blood exert a favorable influence by increasing the filtration pressure. In a similar manner, the aspiratory effect of respiration upon the blood stimulates the circulation, while the respiratory movements of the diaphragm markedly stimulate the resorbent activity of the diaphragmatic pleura and of the peritoneum (lymph stomata). Finally, the activity of the muscles and their coverings (fasciæ, aponeuroses) exerts a great influence on physiological resorption through their continued alternate contraction and relaxation (pressure and aspiration).

In addition to the resorbent mechanism of the blood and lymph vessels, the white blood-corpuscles play an important rôle in resorption. Their part consists in taking up the undissolved constituents of the lymph and parenchymatous fluids (phagocytosis) and on the decomposition and solution of solid tissue masses (histolysis). The phagocytic and digestive activity of the leucocytes is a very important factor in the resorption of pathological products.

The degree of the resorption activity of the individual organs is very different. Resorption is very rapid and intense through the serous membranes with their thin layer of endothelium, their numerous lymph stomata and the underlying extensive plexuses of blood and lymph vessels (pleura, peritoneum, pericardium, pia 
mater,synovial membranes of the articulations and tendon sheaths, tunica vaginalis, iris). Even more pronounced is the resorption power of the lungs with their extraordinary superficial area, their very rich supply of blood and lymph vessels, the thin epithelium of the alveoli with the numerous stomata, and the continuous aspiratory effect of their inspiratory activity. The resorption activity of the mucous membranes differs with their anatomical structure. It is most active in mucous membranes with ciliated (trachea, bronchi) and cylindrical epithelium (intestines, pyloric portion of the gastric mucous membrane, uterus); less active in mucous membranes with pavement epithelium (mouth, pharynx, cesophagus, cardiac portion of the gastric mucous membrane, bladder). The capacity for resorption is least in the unbroken skin, which is only permeable to volatile substances (ether, alcohol, ethereal and alcoholic solutions, chloroform, carbolic acid, ethereal oils and mercury), and also for fats (salves) and soaps when they are rubbed in under pressure. On the other hand, the exposed rete malpighii and corium, with their extensive system of lymph and blood vessels, possess high resorption powers. This is also true of muscle and connective tissue.

Therapeutic Methods.-Fluid and solid pathological products may be removed by supporting or assisting the physiological processes of resorption by mechanical, thermic, chemical (medicines) and electrical methods, or by operative interference. In addition, dietetic, specific and indirect (derivative) methods may also be employed. Those most used are the dietetic, mechanical (exercise, massage), medical (resorbent, rubifacient, derivative and specific drugs) and operative methods.

The dietetic method seeks to bring about the resorption of pathological collections by withholding food (hunger cure) and water (dry cure), by regulating the relative proportion of nutritive substances (reducing treatment) and by increasing metabolism (work).

The mechanical methods are, principally, exercise and massage (see those chapters).

One of the important surgical methods is the artificial pro- 
duction of hyperæmia and inflammation by the application of irritant ointments and by firing (see that chapter).

The derivative method consists of the employment of cathartics (eserine, aloes, salts), sialagogues and diaphoretics (arecoline, pilocarpine), and diureties (digitalis, strophanthus, diuretin, agurin).

Specific resorbents are iodine and potassium iodide (goitre, actinomycosis), mercury and salvarsan (syphilis in man); also the chemical antidotes for poisons.

The operative methods consist in removing pathological fluids by puncture and incision, phlebotomy, etc.

The following group of medicines are usually given the special designation of resorbents in general therapeutics, although the mode of action of the individual drugs is in many instances very different.

\section{RESORBING MEDICINES. RESORBENTS}

Synonyms: Resolvents; discutients; anti-plastics; alterants; dissolving, disintegrating, liquefying, absorbing medicines.

Action and Uses.-Resorbents or resolvents are drugs that bring about an absorption, solution, liquefaction, disintegration or removal of disease products from the tissues or body cavities, chiefly through the medium of the lymph system. None of them exert any direct influence upon the processes of absorption in the lymph vessels, but only indirectly accelerate in different ways the absorption of pathological products present in the body. Some operate by increasing the general metabolism (alkalies), others by causing an outwandering of white blood-corpuscles with the resulting phagocytosis and histolysis (tincture of iodine, blisters), and still others by destroying the infectious agents and killing the pathological tissue cells and instituting a degenerative metamorphosis of the same (potassium iodide, iodoform, mercury, salvarsan). Additional modes of action are: by transforming solid pathological products into a fluid and more readily resorbable form (sodium chloride); by increasing the arterial blood supply (acrics); by causing a thickening of the blood and consequent 
absorption of fluid from the tissues (cathartics, diuretics), and by stimulating the blood circulation (digitalis). The important resorbents are the following:

1. The iodine preparations, potassium iodide, tincture of iodine and iodoform, which are used internally as well as externally. Internally, iodine has a paralyzing and lethal effect upon pathological cell collections, especially the cells of goitre, the cells of the lymph glands, and white blood-corpuscles, causing them to rapidly undergo fatty and granular degeneration, after which they are resorbed and disposed of through the process of metabolism. In addition, iodine has a specific action against actinomycosis (but not against botryomycosis) and the neoplasms of tertiary syphilis of man. In chronic metallic poisonings, iodine acts internally as a resorbent by combining with the almost insoluble metals (mercury, lead) and forming the more soluble and more easily absorbed iodides. When applied externally, the absorbent action of iodine results from the production of an erysipelatous-like inflammation, with outwandering of leucocytes (local leucocytosis and phagocytosis), the absorption of masses of pathological exudates by these cells, and the liberation of enzymes and histolysis.

2. The alkalies (sodium chloride, sodium bicarbonate, sodium sulphate, Carlsbad salts and ammonium chloride) dissolve and liquefy the solid inflammatory products, catarrhal secretions and croupous exudates upon the mucous membranes, serous membranes, in the lungs, liver and other internal organs; increase metabolism, thus increasing resorption; increase diffusion through the animal membranes, especially the vessel walls; stimulate the secretory and excretory activity of the glands, and neutralize and dissolve acids which collect in the body and are precipitated in diseased conditions (uric acid). Externally, the alkalies (soda, potash, lye, soap) also act as resorbents by softening and dissolving the products of chronic inflammation of the skin.

3. Sulphur acts indirectly as an absorbent by stimulating metabolism and increasing the secretions of the glands (liver, cutaneous glands, mucous glands). It is therefore usually em- 
ployed to absorb chronic exudates in lymphadenitis and also in chronic metallic poisonings. The extent of the resorbent action of the antimony salts (black sulphide of antimony, sulphurated antimony) is not definitely known.

4. The mercury salts were formerly regarded as especially active resorbents (antiplastics). A specific resolvent action has been demonstrated for the mercurials only in connection with secondary syphilis of man. The much-boasted absorbent action of gray mercurial ointment is due to the massage and antiseptic effect. The antiplastic properties of calomel are to be attributed to its laxative action. It has been scientifically established that mercury (corrosive sublimate) only in repeated minimum doses increases metabolism, in consequence of which it probably also stimulates resorption. Similar knowledge exists with regard to arsenic.

5. In addition, the cathartics, especially arecoline and aloes, the diuretics, the diaphoretics, the sialagogues, and the cutaneous irritants act indirectly as stimulants to resorption.

Drugs.-1. Potassii iodidum. Potassium iodide. A specific against goitre, actinomycosis and lymphomas; a resorbent of blood extravasations in the brain, inflammatory exudates upon serous membranes, in the lungs, etc., also in chronic metallic poisonings. Dose for horses and cattle, $5-15,3 \mathrm{i}$ to iv; sheep, goats and swine, 1-2, grs. xv to xxx; dogs, $0.25-1$, grs. iij to xv; cats and fowl, $0.1-0.2$, grs. iss to iij.

2. Iodoformum. An internal resorbent in goitre, lymphomas and tuberculosis. Dose for horses and cattle, 2-5, 3ss to j; dogs, $0.05-0.2$, grs. $3 / 4$ to iij.

3. Tinctura iodi. Tincture of iodine. Exclusively an external resorbent in surgical affections: exostoses, periostitis, tendinitis, tendovaginitis, etc.

4. Sodii chloridum, Sodii bicarbonas, sodii sulphas, *sal Carolinum factitium (Carlsbad salts). Resorbents to dissolve solid exudates in the body, solvents of mucus in catarrhs, stimulants to metabolism in obesity. Dose for cattle, $50-100$, 予 iss to iij; horses, 25-50, 3 vj to xij; sheep and goats, 2-5, 3ss to i; swine, 
2-5, 3 ss to i; dogs, 1-2, grs. xv to xxx; cats and fowl, 0.5-1, grs. vij to $\mathrm{xv}$.

5. Ammonii chloridum. Ammonium chloride. A resorbent in bronchitis and croupous pneumonia, croupous pleuritis, peritonitis, etc. Dose for cattle, 10-25, 3ijss to vj; horses, $8-15,3 \mathrm{i}$ to iv; dogs, $0.2-1$, grs. iij to $\mathrm{xv}$.

6. Sulphur lotum, sulphur præcipitatum, sulphur sublimatum. Sulphur. Metabolic stimulants in chronic exudates. Dose for horses and cattle, $2-5$, 3 ss to j; dogs, $0.05-0.2$, grs. $3 / 4$ to iij. 


\section{GENERAL THERAPEUTICS OF FEVER}

Nature of Ferer.-By the term fever is understood a symptomcomplex, in which the general condition is disturbed in several respects. The most important changes are elevation of the body temperature, increased frequency of the pulse, change in the distribution of the blood and of the blood-pressure, alteration of the composition of the blood and derangement of the digestive, respiratory and nervous apparatuses. Increase of the body temperature is not the only symptom of fever.

The exact processes concerned in the generation of fever are not entirely understood. Like inflammation, fever is to be regarded as a natural reaction of the body designed to protect it against invading disease-producing agents. In this connection, the most important rôle seems to be played by the formation of antitoxins (antibodies) on the one hand, and by the heat centre in the brain on the other. The antitoxins neutralize the bacterial poisons (toxins) with the augmentation of albumin decomposition and increase of metabolism. The heat centre regulates the uniform distribution of heat and also the generation of heat in the body (calorific centre). When the heat centre is stimulated the body temperature rises; when it is depressed or paralyzed the temperature falls. The centre may be stimulated in several ways: traumatically, by puncturing with an instrument or wounding it otherwise (heat puncture in rabbits); thermically, by high degrees of heat (heat stroke, sun stroke); chemically, by numerous chemical substances (toxins, ferments, mallein, tuberculin); or reflexly, by pain (so-called nervous fever). On the other hand, the heat centre may be depressed and the body temperature lowered by traumatic (destruction of the centre), thermic (cold) and chemical (acetanilid, antipyrine) influences.

Causes and Forms of Fever.-The causes of fever are various. Bacteria are most frequently the cause (wound fever, infectious fever). Not rarely, however, fever occurs without the inter- 
vention of bacteria from the resorption of products resulting from the disintegration of the blood (aseptic fever).

1. In wound fever, the formation of antitoxic substances is accompanied by increased metabolism, disturbance of heat regulation and stimulation of the heat centre, the result of the resorption of dissolved bacterial toxins and certain chemical substances from the wound secretion. Wound fever is therefore to be regarded as a resorption fever. When the materials absorbed are bacteria or toxins produced by the bacteria in the wound the term septic or bacterial fever is used (infectious fever, intoxication fever, septicæmia, pyæmia). In a large number of cases, fever is associated with entirely benign wounds like those following castration, and non-infected wounds such as subcutaneous bone fractures and blood extravasations. Fever in these cases probably occurs from the resorption of the ferment-like decomposition products of the blood and tissues, the action of which upon the blood and the nervous system is similar to that of the bacterial toxins. A fever of this character is called aseptic or ferment fever. These active chemical substances are blood and tissue ferments (fibrin ferment, histozyme), organic toxins arising from the decomposition of the tissue cells (nuclein from the nuclei of the white bloodcells, free hæmoglobin) and sometimes also glandular secretions.

2. The infectious fever of the acute infectious diseases (contagious pneumonia, strangles, canine distemper, foot-and-mouth disease, etc.) is produced in the same manner as wound fever, the infection and intoxication of the blood occurring usually through the respiratory or gastro-intestinal mucous membrane.

3. As non-bacterial fevers there are yet to be mentioned nervous fever (very painful conditions), rheumatic fever (diseases due to chilling), inanition fever (anæmia), also the fever of heat stroke, fatigue (restraint of horses), and overheating (long rides, casting).

Physiology of Heat.-Animal heat arises from the activity of the body cells, especially the muscle and gland cells, chiefly from the burning up (oxidation) of the carbohydrates. The constancy of the normal temperature is maintained by the regulating activity of the heat centre in connection with the vasomotor nervous sys- 
tem (vasoconstrictors, vasodilators) and the sweat glands. The heat centre, situated in the region of the corpus striatum, regulates heat production and heat dissipation. Increased dissipation of body heat is brought about by dilation of the cutaneous blood vessels and increased secretion of sweat, while contraction of the cutaneous vessels decreases heat dissipation. The regulation of heat dissipation through the skin is of great importance to the body in health as well as in fever because by far the greatest amount (84 per cent.) of heat produced in the body is given off through the skin.

Therapeutic Methods.-The very complicated disease process known as fever can be treated in various ways.

1. The antiseptic, or the causal method, is to be preferred to all others when the microörganisms causing the fever are accessible to the direct action of antiseptics. This is the case in wound fever. The most important points in connection with the treatment of surgical fever are thorough disinfection of wounds, incision of abscesses and phlegmona, amputation of necrotic masses, drainage, change of dressings and irrigation. On the other hand, the antiseptic method cannot be used in the treatment of the acute, febrile, infectious diseases (influenza, strangles, canine distemper, aphthous fever, etc.) because the microörganisms which have invaded the blood usually cannot be destroyed by an antiseptic without danger to the animal body. The action of salvarsan in contagious pneumonia of horses, of quinine in malarial fever and of salicylic acid in articular rheumatism are exceptions. Immunity against some infectious diseases can be obtained by protective vaccination (prophylactic method); this is especially true of swine erysipelas, anthrax, black leg [and hog cholera]. Furthermore, cutaneous irritation, by increasing the physiological production of antitoxins in the body, operates in a certain sense antiseptically.

2. The expective or dietetic method omits the use of antipyretics entirely. The fever of the acute infectious diseases, when running a typical course, is usually not to be combated, but is rather to be regarded as a natural healing process or a protective 
reaction of the body against the invading microörganisms, which are rendered harmless by the high temperature and the increased formation of antitoxins. In addition, attention must be called to the harmful effects of many antipyretics upon the digestion and the nervous system.

So long as the infectious fever does not deviate from its typical course and the nervous system, the heart and the digestive organs are not endangered by an excessively high or a very long continued fever, the expective method is entirely rational. It must, however, be abandoned immediately whenever, because of the type, height or continuance of the fever, the general condition, especially the activity of the heart and brain and the appetite for food, is disturbed.

3. The symptomatic treatment of fever by the use of antipyretics, cardiac stimulants, nerve stimulants, digestive tonics, derivatives (cathartics, diaphoretics, sialagogues, diuretics) and cutaneous irritants is of the most practical importance in veterinary medicine in the treatment of the febrile infectious diseases. Among the different symptoms of fever, a considerable rise of temperature is the most significant and the most dangerous to life. Temperature-reducing drugs (see below) are therefore of first importance in the treatment of fever. In addition, in severe disturbance of the cardiac functions and of the nervous system the administration of cardiacs and nerve excitants is also indicated (see those chapters). The cathartics empty the intestinal canal (infectious material), hasten the elimination of infectious material and toxins from the blood by stimulating the intestinal gland secretions, overcome the constipation frequently present in fever, and also reduce temperature. Reduction of temperature together with a change in the distribution of the blood is produced by cutaneous irritants (oil of mustard, Priessnitz dressing). Phlebotomy, formerly employed for its derivative action, has passed out of use.

4. The bath treatment of fever (thermic method, hydrotherapy) in the form of cold baths $\left(20-30^{\circ} \mathrm{C}\right.$.) is an important method in human medicine, especially in the treatment of abdominal typhus. In veterinary medicine, for technical reasons, this method, as a 
rule, cannot be used. Simple washing or douching with cold water, also wrapping in cold wet sheets, is without considerable value.

\section{MEDICAL ANTIPYRETICS}

Synonyms: Antifebrilics, antithermics, febrifuges, antitypics, antityposics, antiperiodics.

Actions.-The reduction of temperature by antipyretics is brought about in very different ways. The action of most of them is very complicated. According to the mode of action, they may be divided into the following groups:

1. Depression of the heat centre in the brain, when it is excited by fever, with consequent reduction of temperature, appears to be the dominant action of the modern antipyretics: antipyrine, acetanilid, aspirin, phenacetin, salipyrin, lactophenin, pyramidon, etc. They are to be regarded accordingly as narcotics for the heat centre (or the vasomotor centre?). This property is in agreement with the fact that antipyrine, aspirin, etc., also exert a narcotic effect upon other parts of the nervous system, especially in neuralgia and also in the production of local narcosis; the temperature also falls in morphine and chloroform narcosis. The rapid action of the modern antipyretics is in great contrast to that of the other antipyretics, the fall of temperature beginning as early as 10 to 20 minutes after administration, and can only be explained through the action of a promptly functionating heat-regulating centre. Digitalis, the action of which is essentially different, does not reduce temperature until the twelfth to the twenty-fourth hour. The action of the modern antipyretics upon the calorific centre may be demonstrated experimentally. Given to animals with fever resulting from septic infection, antipyrine immediately reduces the temperature so long as the heat centre remains intact. After section of the brain back of the corpus striatum, however, antipyrine has no influence on the temperature.

2. Increasing heat dissipation through the skin. Those antipyretics which by stimulating the vagus centre increase bloodpressure and which by regulating the distribution of the blood 
bring blood from the centre of the body to the periphery, where it can more readily give off its heat to the surrounding atmosphere, reduce the temperature of the body by increasing the dissipation of heat through the skin. The same effect is obtained by other drugs which act upon the cutaneous blood-vessels through the vasomotors (paralysis of the vasomotor centre). The antipyretics which operate by increasing blood-pressure include particularly digitalis and strophanthus. Dilation of the cutaneous blood-vessels is a part of the antipyretic action of alcohol, and also of the modern antipyretics in so far as they depress the anterior portion of the calorific centre (vasomotor ganglion cells for the cutaneous vessels).

3. By decreasing metabolism, partly by retarding the giving off of oxygen by the red blood-corpuscles to the tissues (internal respiration) and partly by depressing the oxidation processes in the cells of the tissues, alcohol, quinine and hydrocyanic acid cause a reduction of body temperature. The acids act as antipyretics by reducing the alkalinity of the blood and thus decreasing its oxidation capacity.

4. By inhibiting and destroying the fever-producing microorganisms, camphor, salicylic acid, quinine, alcohol, arsenic, salvarsan (organic arsenical compound), some of the modern antipyretics and vigorous cutaneous irritation (increased formation of antitoxins) bring about a fall of temperature.

A peculiarity of many antipyretics is that they only reduce the increased temperature of animals in a febrile condition and do not lower the normal body temperature of animals in health. This is readily explained with regard to those antipyretics which reduce temperature by destroying the fever-producing organisms, since in the healthy body there are no such organisms to destroy. The modern antipyretics, which operate as cerebral sedatives, exert a more pronounced narcotic influence upon the heat centre or upon the vasomotor centre irritated by fever than upon the normal heat centre. Those antipyretics which act by decreasing metabolism can lower the body temperature in normal as well as in febrile animals, as has been demonstrated by experiments with large doses of alcohol and quinine. In individual cases, the modern antipyretics 
also reduce normal temperature; this has been demonstrated by Fröhner, especially in connection with acetanilid (Monatshefte für prakt. Tierheilkunde, 1893).

Antipyretics.-1. Acetanilidum. Acetanilid. The cheapest and most powerful modern antipyretic. Dose for horses and cattle, $20-40,3 v$ to $x$; dogs, $0.25-1$, grs. iij to xv. *Aspirin has a similar action. (The patented name aspirin is to be avoided and the chemical name, acidum acetyl-salicylicum, used in prescriptions, as the drug costs one-third less under this name.)

2. Antipyrina. Antipyrine. A very effective but costly antipyretic. Prescribed under the chemical name, pyrazalonum phenyl dymethylicum, it costs one-half less than under the patented name antipyrine. A specific against muscular and articular rheumatism. Dose for horses and cattle, 15-25, 3 iv to vj; large dogs, $2-4$, 3 ss to $j$; small dogs, $0.5-1$, grs. vij to xv; cats, $0.2-0.5$, grs. iij to vij.

3. Acetphenitidinum. Phenacetin. A very good modern antipyretic. Dose, same as for acetanilid. *Lactophenin and *salipyrin have a similar action.

4. Camphora. Camphor. A specific antipyretic for septic fever and fever with heart weakness. Best used subcutaneously in the form of spiritus camphoræ or camphor oil [*oleum camphoratum: camphor 1 , olive oil 10 ; *oleum camphoratum fortior: camphor 1, olive oil 4]. The doses heretofore given of these preparations were much too small. The single dose of oleum camphoratum fortior for the horse, subcutaneously, is 50-150, 兮 iss to v; daily doses, 100-250, 兮 iij to viij.

5. Digitalis. A superior antipyretic in severe infectious fever with cardiac weakness (contagious pneumonia, etc.). Dose (not to be repeated) for horses, $5-10,3 i$ to ijss; dogs, $0.2-0.5$, grs. iij to vij.

6. Acidum salicylicum. Salicylic acid. A specific against acute muscular and articular rheumatism; not effective in septic and pyæmic fevers. Dose of salicylic acid and sodii salicylas for horses and cattle, $25-75,3$ vj to 5 ijss; swine, $2-5$, 3 ss to j; dogs, $0.25-2$, grs. iv to $\mathrm{xxx}$. 
7. Alcohol. A valuable antipyretic in many fevers, especially septic and pyæmic fevers. Acts at the same time as a stimulant to the brain and heart and also as a food (economizes tissue waste). Dose for febrile horses and cattle, 100-200, 争ij to vjss, every two hours; sheep, goats and swine, 25-100, 3 vj to 5 iij; dogs, 25-50, $3 \mathrm{vj}$ to xii; cats, $2-5,3$ ss to j. Dose of brandy (spiritus vini gallici) two to three times these quantities.

8. Quinina. Quinine. Specific against intermittent fever. Unreliable in contagious pneumonia, influenza, canine distemper, articular rheumatism, etc. Dose of quininæ sulphas for horses and cattle, $10-25,3$ ijss to vj; dogs, $0.25-1$, grs. iij to xv. 


\section{GENERAL THERAPEUTICS OF THE DISEASES OF THE NERVOUS SYSTEM}

Pathology. - Of the diseases of the brain, spinal cord, and peripheral nerves, those of especial therapeutic consideration are acute inflammation and hyperæmia of the brain, Bornas's disease of the horse, epilepsy and eclampsia, sturdy or gid, cerebrospinal meningitis, inflammation of the spinal cord, trotting disease of sheep, and the spinal paralyses; also paralyses of the peripheral nerves, especially the optic, facial, trigeminus, suprascapular and radial. The central nervous system is also frequently affected secondarily in the course of infectious diseases (canine distemper, tetanus, contagious pneumonia, strangles, tuberculosis, dourine), in poisoning (strychnine, lead, alcohol, red poppy, equisetum) and in wounds (fracture of the skull or vertebræ, hemorrhage, concussion, contusion).

Physiology.-The most important part of the brain physiologically and therapeutically is the cerebral cortex. This is, in the first place, the location of the psychic functions (consciousness, memory, intelligence, volition). In the cerebrum are situated the motor, sensory, optic and acoustic cortical areas. The most of these cortical centres manifest a specific relation to certain drugs. They may also be stimulated by mechanical, thermic and electrical stimuli, and by the will. The corpus striatum is the chief seat of the heat centre. In the corpora quadrigemina and optic thalamus is the pupillary centre, by which stimuli acting upon the retina are transmitted reflexly to the oculomotor nerve (pupillary motion). In addition, the middle brain (corpus striatum, optic thalamus) contains centres for the coördination of movement, which also originate forced movements. The cerebellum contains the centre for the muscle sense and the equilibrium of the body (centre of equilibrium); its extirpation produces cerebellar ataxia. The semicircular canals are to be regarded as static organs for the movement and carriage of the head. Ganglion cells form the 
anatomical foundation of all of the nerve centres (psychic, automatic, reflex activity).

The medulla oblongata also contains a large number of important vital centres. These centres can also be influenced therapeutically by specific drugs. Especially noteworthy are the respiratory, vasomotor, vagus, salivary, sweat and diabetic centres, and the centres of vomition and swallowing.

In the spinal cord are the paths of conduction of the motor (inferior column) and the sensory nerves (superior column) of the extremities; also the centres for the uterus, the vessels, the sweat glands, the discharge of urine, the erection of the penis and for the sphincter muscle of the anus.

The peripheral nerves have sensory, motor, secretory, vasomotor, trophic (?), reflex-stimulating and reflex-inhibiting functions. Especially important in therapeutics are the reflex functions (centripetal, centrifugal nerves; reflex movement, reflex inhibition, reflex secretion). All peripheral nerves, but especially the sensory nerves of the skin and mucous membranes, and all. parts of the central nervous system possess reflex powers,--i.e., the ability to transmit a centripetal stimulus to a reflex centre and out over a centrifugal path without the intervention of consciousness (coughing, sneezing, pupillary contraction, vascular contraction, vascular dilation, perspiration, vomiting, increased peristalsis, tendon reflexes).

The intrinsic chemical constituents of the brain and nerves are the phosphorus-containing protagon and lecithin, cerebrin, and other proteids. Resting nerve substance is alkaline in reaction; the active, acid (lactic acid and other products of fatigue). Natural sleep is brought about by the action of the fatigue products on the albumin of the ganglion cells in a manner similar to that of morphine and chloroform narcosis; anæmia of the brain occurs secondarily.

Therapeutic Methods.-1. On account of the varied and intimate relations between the nervous system and a large number of drugs, the medical treatment of nervous diseases is a prominent feature of the therapeutics of nervous diseases. The action of 
drugs, it is true, is in most instances only indirect and symptomatic, and is either sedative or stimulant. If, for example, the psychic excitement in the course of inflammation of the brain is relieved by morphine, the disease process itself, i.e., the inflammation of the cerebral membranes, remains unaffected. There are, however, also cases in which there is a direct influence exerted by the drug upon diseases of the nervous system (morphine in eclampsia, potassium bromide in epilepsy, strychnine in amaurosis, physiological antidotes).

2. The derivative method is also partly medical. It consists in the employment of the cathartics, sialagogues and diaphoretics, blisters and phlebotomy (indicated in cerebral congestion).

3. Electrotherapy is also of value in veterinary medicine, especially in canine practice, for the treatment of peripheral and spinal paralyses. The faradic current stimulates the spinal cord reflexly in the same manner as cutaneous irritation; the constant current acts directly, like the drugs acting upon the central nervous system.

4. Massage is an important mechanical method, especially for peripheral paralyses (tapotement).

5. Hydrotherapy is practicable only to a limited extent in veterinary medicine (cold douche, ice poultice, Priessnitz dressing).

6. Operative treatment is undertaken in sturdy or gid and fractures of the skull (trepanation); also in the form of neurotomy.

Finally, there is the rest treatment (protection from external stimuli, quiet and dark stall), and the dietetic (green food in cerebral inflammation in the horse), the expective (cerebral hemorrhage) and the prophylactic methods. The hypnotic and suggestive treatment, very important in human medicine, cannot be used on the domestic animals.

Drugs Acting on the Nerves. Neurotics. Nervines

Classification.-The drugs used in the treatment of diseases of the nervous system may be classified according to the part upon which they act as well as according to the character of the action. Grouped according to the part acted upon, there are drugs acting 
upon the brain (cerebralics, encephalics), upon the spinal cord (spinalics), and upon the peripheral nerves (peripheric nervines). Besides this anatomical division, the classification based upon physiological principles is of special practical importance in therapeutics; according to action, there are drugs which are stimulating (excitants) and others which are quieting (sedatives) to the nervous structures. Finally, there may be a combination of the two methods of classification. In this way the following subdivisions have been formed:

I. Cerebralics. These are again subdivided into drugs which act upon the cerebrum, upon the medulla oblongata and upon the twelve cranial nerves. Those drugs which have a relation to the cerebellum, e.g., alcohol and aspidium, are of no special practical consideration in this connection.

1. The drugs acting upon the cerebrum are divided according to the location and character of their action into the following groups:

(a) Excitants to the cortical psychic centres: alcohol, ether, caffeine, nicotine, cocaine and apomorphine.

(b) Excitants to the motor centres: atropine, camphor, picrotoxin and lead salts.

(c) Sedatives for both kinds of centres: potassium bromide and chloral hydrate.

(d) Hypnotics (drugs that produce sleep): morphine, chloral hydrate, sulphonal, trional, veronal, medinal, isopral and adalin.

(e) Narcotics and anæsthetics in the restricted sense: chloroform, ether and chloral.

2. For the medulla oblongata there are:

(a) Excitants in general: veratrine, atropine, hyoscine (scopolamine), caffeine, strychnine, camphor and ammonium salts.

(b) Sedatives in general: morphine, potassium bromide and chloral hydrate.

(c) Excitants to the respiratory centre: atropine, hyoscine (scopolamine) and strychnine. 
(d) Excitants to the vasomotor centre: caffeine, theobromine, theocine and digitalis.

(e) Depressor to the vasomotor centre: amyl nitrite.

(f) Excitants to the vagus centre: digitalis and strophanthus.

(g) Excitants to the centre of vomition: apomorphine and emetine.

3. The specifics for the cranial nerves are:

(a) Olfactory: strychnine.

(b) Optic: strychnine, quinine, santonin, aspidium and amyl nitrite.

(c) Oculomotor: the mydriatics - atropine and hyoscine (scopolamine); the myotics-eserine, pilocarpine and arecoline.

(d) Trochlear (and abducens): santonin.

(e) Trigeminus: strychnine, aconitine and cocaine.

(f) Facial: strychnine.

(g) Auditory: quinine, salicylic acid and cannabis indica.

(h) Glossopharyngeal: apomorphine and atropine.

(i) Vagus: atropine, hyoscine (scopolamine), the digitalis group, strychnine and veratrine.

(k) Hypoglossal: strychnine.

In addition, atropine, ergot, cocaine and amyl nitrite are specific for the sympathetic, and atropine and morphine for the splanchnic nerves.

II. Spinalics are divided, according to their action, into:

(a) Tetanics: strychnine and cornutine (lumbar cord).

(b) Sedatives: morphine, potassium bromide and chloral hydrate.

III. Peripherics. These are classified according to the functions of the peripheral nerves as follows:

(a) Motor excitants: camphor and eserine.

(b) Motor sedatives: curare and conine.

(c) Sensory excitants: veratrine and cutaneous irritants.

(d) Sensory sedatives or local anæsthetics: cocaine, adrenalin, novocaine, anæsthesin, eucaine B, tropacocaine, alypin, 
stovain, propæsin, dipropæsin, subcutin, zycloform, orthoform, nirvanin, acoin, holocain, antipyrine, menthol and ether; also morphine and hydrocyanic acid.

For the purposes of general therapeutics, division of the neurotics simply into stimulants and sedatives is most desirable. Both classes of drugs operate by producing a specific change in the nerve albumins, consisting especially in the formation of double combinations, as morphine albuminate, strychnine albuminate and bromide albuminate. Some of these combinations are less susceptible to stimuli (morphine albuminate) than the original nerve albumin. In some instances, changes in the composition and in the distribution of the blood also participate in the action.

1. STIMULANTS TO THE NERVOUS SYSTEM. EXCITANTS

Synonyms: Analeptics, stimulants, euphorics, exhilarants, tetanics, spasmodics, spinants, hyperkinetics, epileptifacients, antiparalytics; exciting, stimulating, restorative, invigorating medicines.

Uses.-The nerve stimulants are employed in the treatment of the various disease conditions of the nervous and muscular systems which are inaugurated with general or local weakness or paralysis of these systems or of individual parts: brain, spinal cord, peripheral and sympathetic nerves; also the skeletal muscles, heart, intestinal musculature, etc. The most important of these diseases are:

1. Psychic depression in the course of severe febrile conditions, e.g., influenza and contagious pneumonia of the horse, canine distemper, catarrhal fever of cattle, swine erysipelas, fowl cholera and septic and pyæmic conditions.

2. General weakness in the course of acute and chronic diseases, e.g., parturient paresis of cattle, anæmia, leukæmia, after difficult parturition, following overexertion, after internal and external hemorrhages, and in fall of body temperature.

3. Heart weakness in the course of infectious diseases, e.g., contagious pneumonia and malignant aphthous fever.

4. Brain diseases in the stage of depression: acute inflammation of the brain, chronic hydrocephalus, cerebral anæmia, vertigo, syncope, cerebral apoplexy and cerebral paralysis. 
5. Pareses and paralyses of the cranial nerves, especially incipient paralysis of the optic (amblyopia and amaurosis), recurrent (roaring in horses), facial (paralysis of the lower lip), trigeminus (paralysis of the muscles of mastication), and hypoglossal (paralysis of the tongue).

6. Spinal paralyses. These include the different forms of weakness of the loins; spinal meningitis and myelitis; concussions, hemorrhages, traumatic affections of the spinal cord, paralytic conditions of the posterior quarters, and paralyses of the bladder, rectum, penis and tail. Of the infectious diseases which are complicated with spinal paralyses, canine distemper, contagious pneumonia and dourine of horses, and trotting disease of sheep may be mentioned.

7. Muscle paralyses. These are chiefly observed in the horse in the course of azoturia, after casting, in inflammation of the muscles and in some poisonings (lead).

8. Paralyses of peripheral nerves. Of these, paralyses of the facial, radial, subscapular, sciatic, tibial, peroneal and obturator nerves are to be especially mentioned.

9. Paralytic conditions in regions supplied by the sympathetic. To this class belong pareses of the muscular wall of the rumen in ruminants and of the intestinal canal in horses.

10. Poisonings. In most poisonings the use of an excitant is necessary because in the later stages paralytic symptoms appear which must be treated symptomatically. Here may be especially mentioned poisonings from phosphorus, arsenic, mercury, lead, carbolic acid, alcohol, chloroform, colchicum, opium and morphine, red poppy, agrostemma githago, chick pea, taxus, digitalis, aloes, lupines, equisetum, cantharides, snake bites and toxins (meat and mushroom poisoning).

Drugs.-In veterinary practice, the most frequently used excitants are the alkaloids (which are the most powerful stimulants), alcoholic and ethereal preparations, ethereal oils and inorganic compounds. The most important are given below in the order of their strength:

1. Strychnina. Strychnine. The principal remedy in spinal 
motor paralyses, pareses and paralyses of the posterior quarters, paralysis of the recurrent nerve (roaring), paralysis of the bladder, penis, tail and rectum, amblyopia and amaurosis, lead paralysis, and chloroform, chloral hydrate and other narcotic poisonings. The dose of strychninæ sulphas internally and subcutaneously (not intratracheally) is one-tenth of a milligram for each kilogram of body weight; therefore, the dose for horses is $0.05-0.1$, grs. $3 / 4$ to iss; cattle, $0.05-0.15$, grs. $3 / 4$ to $\mathrm{ij}$; sheep and goats, 0.005 , gr. $3 / 40$; swine, $0.002-0.005$, gr. $1 / 40$ to $3 / 40$; dogs, $0.001-0.003$, gr. $1 / 70$ to $1 / 25$; cats, $0.0005-0.001$, gr. $1 / 140$ to $1 / 70$; fowl, 0.0002 0.0005 , gr. $1 / 340$ to $1 / 140$.

2. Veratrina. Veratrin. The chief remedy in fatigue and paralyses of the striated and smooth musculature of the body; also a very powerful excitant to the entire nervous system. It is used principally in cattle practice, in which it is employed especially in the treatment of antepartum paralysis, exhaustion after parturition, parturient paresis and atony of the musculature of the rumen. In addition, it is frequently prescribed in heart weakness and in conditions of collapse, especially in the course of narcotic poisonings. The subcutaneous dose of veratrin in alcoholic solution for horses and cattle is $0.05-0.2$, grs. $3 / 4$ to iij; sheep and goats, $0.01-0.02$, gr. $1 / 8$ to $1 / 4$; dogs, $0.001-0.005$, gr. $1 / 70$ to $3 / 40$; cats, 0.001 , gr. $1 / 70$. The crude drug, veratrum, and tinctura veratri (dose per os for horses and cattle $5-10,3 \mathrm{i}$ to $\mathrm{ijss}$ ) are less frequently used than the alkaloid because of their slower action.

3. Atropine and hyoscine (scopolamine). These are among the strongest nerve stimulants and are specific in heart weakness, threatened respiratory paralysis, and in poisoning by chloroform, morphine, eserine, arecoline and pilocarpine. Dose of atropinæ sulphas for horses and cattle, $0.05-0.1$, gr. $3 / 4$ to iss; sheep and goats, $0.01-0.05$, gr. $1 / 8$ to $3 / 4$; swine, $0.01-0.03$, gr. $1 / 8$ to $1 / 3$; dogs, $0.005-0.05$, gr. $3 / 40$ to $3 / 4$; cats, $0.002-0.005$, gr. $1 / 40$ to $3 / 40$. The dose of hyoscinæ hydrobromidum subcutaneously is onetenth these quantities: horses and cattle, $0.01-0.05$, gr. $1 / 8$ to $3 / 4$; $\operatorname{dogs}, 0.005$, gr. $3 / 40$.

4. Caffeina. Caffeine. A specific against heart weakness, 
incipient oedema of the lungs, spinal paralysis, conditions of collapse, parturient paresis, and poisoning by morphine, chloroform, alcohol and toxins. The subcutaneous or internal dose of *caffeinæ sodio-salicylas, N.F., for horses and cattle is $5-10,3 i$ to ijss; large dogs, $0.5-2$, grs. vij to $\mathrm{xxx}$; small dogs, $0.1-0.5$, grs. iss to vij. An infusion of coffee or tea may be used instead of caffeine.

5. Physostigmine (eserine), pilocarpine, arecoline. Stimulants in relaxation and paralysis of the musculature of the stomach and intestines, especially in cattle and horses. Physostigmine (eserine) is also a myotic (pupillary contraction). The dose of physostigminæ salicylas and physostigminæ sulphas subcutaneously for horses is $0.05-0.1$, grs. $3 / 4$ to iss; cattle, $0.1-0.2$, grs. iss to iij. Dose of pilocarpinæ hydrochloridum for horses, 0.5 , grs. vij; cattle, $0.5-0.8$, grs. vij to xii. Arecolinæ hydrobromidum is given to horses in doses of $0.02-0.08$, grs. $1 / 4$ to $\mathrm{i}$.

6. Camphora. Camphor. One of the most valuable stimulants for the brain and heart in all conditions of psychic depression, stupor, collapse, heart weakness and exhaustion in the course of febrile infectious diseases, severe constitutional affections and narcotic poisonings. It is usually administered subcutaneously to all the domestic animals in the form of camphor oil [camphor 1, olive oil 10; also camphor 1, olive oil 4] or camphor spirit. The single dose of oleum camphoratum fortior [camphor 1, olive oil 4] for horses is $50-150,5$ iss to $\mathrm{v}$; dogs, $5-10$, $3 \mathrm{i}$ to ijss; cats, $1-5$, mexv to 3 i.

7. Alcohol and ether. In small doses both are strong stimulants to the brain and the other parts of the nervous system; also to the heart and digestion. Given subcutaneously or by the mouth in all conditions of weakness or collapse occurring in the course of severe infectious diseases and in inanition, in pronounced exhaustion following parturition and hemorrhage, in severe poisonings and in threatened cerebral and cardiac paralysis. Dose of alcohol for horses and cattle, 25-50, $3 \mathrm{vj}$ to xij; sheep and goats, 10-20, 3 ijss to iv; swine, 5-10, 3 i to ijss; dogs, 2-5, 3 ss to i; cats and fowl, $1-2$, $\pi x v$ to $x x x$. Dose of ether one-half. Alcohol dilutum, spiritus vini gallici, vinum album, vinum rubrum, *beer and 
spiritus ætheris may also be used as stimulants in corresponding doses.

8. Ammonium compounds. These are very powerful excitants to the brain, heart and intestines in all weak conditions in the course of infectious diseases and poisonings, and are sometimes used in the treatment of cattle and the smaller ruminants and dogs. Aqua ammoniæ, diluted with plenty of cold water, is given to cattle in doses of $10-30,3 \mathrm{ij}$ to $\overline{3}$; ammonii carbonas: cattle, $20-50,3 \mathrm{v}$ to xij; sheep and goats, $2-5,3$ ss to $i$; spiritus ammoniæ aromaticus: dogs, $0.3-0.4$, mo to $3 \mathrm{i}$.

9. Potassium salts. These are stimulants to the heart, brain and muscles and are most frequently given to dogs in the form of meat extracts in all weak conditions. Dose, $2-10,3 \mathrm{i}$ to ijss.

\section{DRUGS THAT CALM THE NERVES. SEDATIVES}

Synonyms: Sedantics, narcotics, anæsthetics, temperantics, anodynes, analgics, analgesics, hypnotics, somniferics, soporifics, ebriantics, paralyzants, paregorics, antitetanics, antispasmodics, anticonvulsives, antiepileptics, antineuralgics, anti-asthmatics, antodontalgics; soothing, benumbing, narcotizing, anæsthetizing, pain-alleviating, convulsion-alleviating drugs; drugs that produce sleep.

Uses. - The nerve sedatives are used to overcome conditions of psychic, sensory, and motor excitement in the different parts of the nervous system; also in operations. The diseased conditions in which they are indicated are, in general, the following:

1. Psychic excitement in the course of diseases of the brain and severe febrile general affections, including acute cerebral hyperæmia, acute and subacute inflammation of the brain in the stage of excitement, erethistic affections in the course of chronic hydrocephalus, the cerebral form of canine distemper and psychic disturbances in the course of contagious pneumonia, strangles, malignant catarrhal fever, tuberculosis, gid (stage of immigration), and septicæmia.

2. Cerebral convulsions in the form of epilepsy, eclampsia, catalepsy, chorea, epileptiform convulsions and forced movements. These occur either as primary diseases or secondarily in the course 
of inflammation of the brain, canine distemper, contagious pneumonia, cerebrospinal meningitis, tuberculosis, strangles, and in invasion of the brain by parasites (conuri, echinococci).

3. Spinal convulsions. The rigid cramp of tetanus and the convulsions occurring in the course of spinal meningitis, cerebrospinal meningitis, and trotting disease of sheep may be mentioned as examples. A disposition to take fright readily is also an indication of spinal excitement.

4. Excited conditions of the peripheral nerves. The sensory nerves are in a condition of excitation in the different painful conditions of external and internal organs: colic, cough, neuritis, hyperæsthesia, pruritis, nymphomania, satyriasis; the motor nerves, in peripheral muscular spasms, cramp of the sphincters, straining after prolapse, spasms of the stomach, intestines and uterus, and palpitation of the heart.

5. Poisonings. The most important intoxications accompanied by symptoms of excitation, and which are therefore to be combated with sedatives, are poisoning by strychnine, veratrin, atropine, hyoscine, aconitin, nicotine, cocaine, apomorphine, lead, Hering's lake, alcohol, red poppy and aspidium.

6. Finally, the sedatives are used to prepare animals for operations (see the chapter on general and local narcosis, p. 101).

Classification.-The sedatives are divided into several subdivisions, according to the purposes for which they are used, as follows:

1. Sedatives in a general sense. These include potassium bromide, chloral hydrate, [cannabis indica], opium, morphine, codeine, peppermint, matricaria and valerian.

2. Hypnotics: sulphonal, trional, veronal, medinal, adalin, hypnone, morphine and codeine.

3. Narcotics or anæsthetics, which are again divided into general and local. General: chloroform, [chloral], ether, ethyl bromide, etc. Local: cocaine, adrenalin, novocaine, anæsthin, eucaine B, alypin, tropacocaine, stovain, propæsin, subcutin, zycloform, orthoform, holocain, nirvanin, antipyrine, morphine, ether, menthol, freezing mixtures, etc. Some of the local anæs- 
thetics produce first a powerful local irritation and are therefore called anæsthetica dolorosa.

4. Antispasmodics: potassium bromide, chloral hydrate, morphine and the ethereal oils.

In human medicines there are also other subdivisions; the antodontalgics, or toothache remedies (chloroform, cocaine, adrenalin, dilute carbolic acid, alcohol, brisk embrocations upon the gums and caustics for the dental pulp), the anti-asthmatics, or asthma remedies (chloroform, morphine, amyl nitrite, and lobelia), and the antineuralgics, or neuralgia remedies, (morphine, antipyrine, menthol, migrainine, etc.).

Drugs.-1. Morphine. This is the chief narcotic in operative surgery in dogs; also, a sedative in all conditions of psychic excitation; a local and general anæsthetic in painful conditions, especially colic and cough; a specific antispasmodic in eclampsia of suckling bitches, and an antidote in poisoning by atropine, hyoscine and strychnine. The dose of morphinæ sulphas, subcutaneously, for colic in horses is $0.3-0.6$, grs. iv to ix; as a narcotic in dogs, $0.02-0.15$, grs. $1 / 4$ to ij. Opium was formerly used instead of morphine. The treatment of colic in horses with large doses of tincture of opium $(250,5$ viij) did not prove satisfactory in the Berlin clinic. [American veterinarians have found cannabis indica of more value than opium in controlling pain in colic in horses. The fluidextractum cannabis indicæ is given by the mouth in doses of $15,7 \mathrm{ss}$, repeated every half hour, or in single doses of 4, 3i, intravenously.] Several derivatives of opium and morphine: codeine, heroin, dionine, peronine, etc., are recommended as hypnotics and sedatives.

2. Potassii bromidum. Potassium bromide is a specific against epilepsy, epileptiform convulsions, tetanus, strychnine poisoning and eclampsia; sedative in all conditions of excitation of the brain, in nymphomania, cough and palpitation of the heart. Single doses for horses and cattle, 20-100, $3 \mathrm{v}$ to 5 iij; sheep and swine, 2-5, grs. $\mathrm{xxx}$ to $3 \mathrm{i}$; dogs, $0.25-2$, grs. iij to $\mathrm{xxx}$; cats, $0.25-0.5$, grs. iij to vij; fowl, $0.1-0.2$, grs. iss to iij.

3. Chloroformum. A valuable narcotic in operations on horses; 
antispasmodic in eclampsia, tetanus, strychnine poisoning, and prolapse of the uterus and rectum; local anæsthetic. Dose by inhalation for horses, $50-150$, 兮 iss to $5 \mathrm{v}$. Ethyl bromide is also recommended for inhalation narcosis.

4. Chloralum hydratum. Chloral hydrate. A valuable narcotic in operations on horses; given before casting in the form of clysters (75-125, 筩ijs to $5 \mathrm{jv}$, in warm, mucilaginous fluid). Hypnotic and sedative in all conditions of psychic excitation and painful affections. Specific antidote against strychnine tetanus; antispasmodic in tetanus, eclampsia, convulsions of canine distemper and straining after prolapses. Single doses for horses and cattle, $25-50$, 3 vj to xij; dogs, 0.5 to 5 , grs. vij to $3 \mathrm{i}$; sheep, goats and swine, $2-5$, 3 ss to $i$; cats and fowl, $0.25-2$, grs. iij to xxx. Chloralformamidum, *acetal, *somnal, *bromal hydrate, butyl-chloral and many other new preparations are recommended as substitutes for chloral.

5. Ether. Ether. In large doses, a narcotic for operations, frequently in combination with chloroform. An antispasmodic in spasmodic colic and a local anæsthetic. Dose for cats as an inhalation narcotic, $20-30,3 \mathrm{v}$ to $5 \mathrm{i}$. As an antispasmodic, internally, for horses and cattle, $25-50,3 \mathrm{vj}$ to xij; dogs, $2-5$, $3 \mathrm{ss}$ to $\mathrm{i}$. Alcohol in large doses is also used as a sedative in prolapse of the uterus and rectum. Horses and cattle receive 500-1000, Oi to $\mathrm{ij}$, of spiritus vini gallici (brandy) [or one-half to one pint of alcohol].

6. Cocainæ hydrochloridum. The most valuable local anæsthetic, especially in the treatment of the eye; also a local anæsthetic in operations (Schleich's endermatic method and subcutaneously), and an unexcelled agent in the diagnosis of lameness in the horse $(0.2$, grs. iij, subcutaneously over the nerves on the inside and outside of the fetlock joint, etc., at the place for neurectomy). As substitutes for cocaine, the following have been recommended: *adrenalin, *novocaine, *anæsthesin, *alypin, *stovain, *propæsin, *subcutin, *zycloform, *orthoform, *eucaine, *acoine, *holocain, *nirvanin, *tropacocaine and others, but they are not necessary in veterinary medicine. 
7. Sulphonmethanum. Sulphonal. A good hypnotic for the dog. Dose for large dogs, 2-4, 3 ss to j; small dogs, 0.5-2, grs. vij to $\mathrm{xxx}$. Other hypnotics for animals are sulphonethylmethanum (trional), *tetronal, *veronal, *medinal, *adalin, *acetophenone (hypnone), æthylis carbamas (urethane) and *amylene hydroxide.

8. Ethereal oils. Many of these in large doses possess antispasmodic powers and are for this reason used in spasmodic colic; also used sometimes as local anæsthetics. The most important drugs containing these oils are matricaria, valeriana, mentha piperita and asafœida. Dose of these aromatic drugs for cattle, 50-100, 步iss to $5 \mathrm{iij}$; horses, $25-50,3$ vi to xij; given in infusion. One of the ethereal oils is oleum terebinthinæ.

9. Acidum hydrocyanicum. Used chiefly as a sedative for the sensory nerves of the larynx (cough). [Dose of acidum hydrocyanicum dilutum for horses and cattle, 1.3-4, ngxx to $3 \mathrm{i}$; dogs, 0.06-0.3, mi to v.] Aqua amygdalæ amaræ (bitter-almond water), a preparation containing hydrocyanic acid, is given to horses and cattle in doses of $10-50,3$ ijss to 5 iss; dogs, $0.5-3$, mevij to xlv.

\section{NARCOSIS AND ANÆSTHESTA}

General Narcosis.-The narcotizing of animals for the purpose of performing operations without pain may be general or local. Different drugs are used to produce narcosis in animals of the different species. Chloroform is in general suitable only for horses and is not entirely without danger for these animals. The use of chloroform on dogs, cattle, sheep, goats and cats is not recommended on account of the dangerous complications it produces (paralysis of respiration, convulsions, tympanites, vomiting and traumatic pneumonia). Chloral hydrate is the best narcotic for horses and is to be preferred to chloroform and morphine especially; given per os it produces narcosis very rapidly, per rectum somewhat more slowly; the narcosis is of medium degree, but is sufficient for most operations; chloral may also be employed for animals of other species. Morphine is adapted for use as a general 
narcotic for dogs, injected subcutaneously; less suitable for horses; ruminants are strongly excited by morphine. Ether is used as a narcotic for cats [in the surgical clinic at Copenhagen it is used for cattle; it is also frequently used for dogs].

Local Anæsthesia.-The principal local anæsthetic for all domestic animals is cocaine (ophthalmology, cutaneous injections in the form of infiltration anæsthesia, subcutaneous injection, lumbar injection, rectal mucous membrane, nasal mucous membrane, etc.). More frequently than for therapeutic purposes, cocaine is injected subcutaneously in horses for diagnostic purposes (differential diagnosis of lamenesses). The local action of cocaine is assisted, and its toxicity is at the same time decreased, by the addition of adrenalin (suprarenin) to the cocaine solution. The newer local anæsthetics: novocaine, tropacocaine, holocain, acoin, eucaine $B$, anæsthesin, alypin, stovain, nervanin, orthoform, zycloform, propæsin, etc., have, with the exception of alypin, not displaced cocaine. Ethyl chloride has replaced ether as a local anæsthetic. Anæsthesia by cold (compressed carbonic acid) is little used in veterinary medicine.

The history of general and local anæsthesia has been written by Regenbogen (Monatshefte für praktische Tierheilkunde, Bd. xx, 1909). 


\section{GENERAL THERAPEUTICS OF THE DISEASES OF THE RESPIRATORY APPARATUS}

Pathology.-Of the numerous diseases of the respiratory organs, those which are the object of veterinary treatment include nasal, laryngeal and bronchial catarrh; the more severe forms of rhinitis, laryngitis, tracheitis and bronchitis; œdema of the glottis, œdema of the lungs, roaring, active and passive hyperæmia of the lungs, the different forms of inflammation of the lungs, emphysema of the lungs and pleuritis. More rarely parasitic diseases (lung worm epidemics, pentastomes, œstrus larvæ) and tumors (nasal cavity, larynx) are presented for treatment. On the other hand, secondary inflammatory processes occur very frequently in the respiratory mucous membrane in the course of infectious diseases (strangles, contagious pneumonia, influenza, canine distemper, malignant catarrhal fever, etc.).

Physiology.-The natural protectives of the respiratory organs are the layer of mucus which covers the mucous membrane and the ciliated epithelial cells of the membrane, the cilia of which carry out by their movement any foreign bodies which may enter. At the same time, the air passages with their extensive surface and recesses serve to warm the air and filter out the dust. Finally, for the forcible removal of foreign bodies and disease products there are certain defensive reflexes (sneezing, coughing).

The pulmonary respiration, to which the cutaneous respiration can be to a certain degree compensatory, consists of an interchange of gases between the blood and the external air (carbon dioxide, oxygen). This gaseous interchange is facilitated by the continual change of air (inspiratory diaphragmatic contraction, expiratory lung elasticity), the extensive surface of the lungs (in the horse 100 times the body surface) and the very thin diffusion membrane (alveolar walls). In diseases of the lungs, these principal factors of respiration are more or less interfered with (pneumonia, emphysema). 
The automatic respiratory centre in the medulla oblongata can be stimulated directly and indirectly by different stimuli, especially drugs. Insufficient oxygen and excess of carbon dioxide in the blood (anæmia, venous hyperæmia, muscular activity), febrile temperature, and atropine, scopolamine and other drugs act as direct stimulants. Indirectly, or reflexly, the respiratory centre is stimulated by stimulation of the peripheral ends of the vagus nerve (regulating nerve of respiration) in the lungs, the cutaneous sensory nerves (painful trauma, inflammation, cold), the superior laryngeal nerve (cough), the trigeminal, olfactory and other peripheral nerves. The artificial stimulation of the respiratory centre is very important, for example, in chloroform asphyxia (atropine, scopolamine, slapping the skin in the region of the flanks, cold douches).

Therapeutic Methods.-1. In the treatment of diseases of the organs of respiration, the most important therapeutic measure is a provision for a supply of pure air. Good ventilation of the stable or, when possible, keeping the patient in the open air (pasturing, bivouac, staking) is alone sufficient to effect a cure in many cases. This is especially true of the catarrhal and infectious diseases of the organs of the chest. Experience in the Prussian army has in numerous instances shown the favorable effects of bivouacking in summer, particularly in contagious pneumonia in horses. Moist air (warm winds) is also very healing in catarrhs of the respiratory mucous membrane; it promotes the liquefaction of the catarrhal secretions. In contrast with this is the drying and heat-extracting influence of cold winds. With the air therapy should be combined proper care of the skin (cutaneous respiration).

2. Drugs may be employed for different purposes. Of special consideration are the so-called expectorants, i.e., drugs which promote the throwing off, or expectoration, of abnormal substances from the respiratory tract (see'p. 107). Also of importance are the derivative (cathartics, diuretics and sialagogues) and the symptomatic methods (narcotic cough remedies) of treatment, the use of stimulants to the respiratory centre, and the astringent, antiseptic and antiparasitic methods (catarrhs, infectious diseases, lung worm epidemics). 
3. The inhalation method (steam, medicinal solutions, powders, gas) has for its purpose the local treatment of the diseased respiratory mucous membrane. It has fallen into disuse because recent experiments have shown that vapors, when inhaled with the usual inhalation apparatus, do not reach the alveoli of the lungs but are held back by the nasal septum, the posterior wall of the throat, the epiglottis and the vocal cords. With the very complicated respiratory apparatuses (Bulling's, Siegle's, Wassmuth's and Schenk's) used in human medicine, by which the medicament is divided into a very fine spray, the vapor only reaches the alveoli when it is aspirated through the mouth and does not arrive in that part of the respiratory tract when inhaled through the nose. On account of their complicated structure and cost, these apparatuses are of no practical value in veterinary medicine. Inhalations of oxygen (contagious pneumonia of horses) have not proven of value in the treatment of animals. Chloroform and ether are given by inhalation for their general effects.

4. The hydropathic method and the method of cutaneous irritation are very effective in different ways. The stimulant effect upon the cutaneous respiration, the reflex stimulant action upon the respiratory centre, the derivative effect upon pulmonary inflammation and pleuritis, and the anodyne action (cough) and antipyretic effect of the Priessnitz dressing and rubefacients or vesicants (oil of mustard in alcohol applied to the chest walls) are especially worthy of mention.

5. The operative method includes phlebotomy (pulmonary congestion), tracheotomy (inspiratory dyspncea in consequence of nasal, pharyngeal and laryngeal stenosis), thoracocentesis (hydrothorax, empyema) and extirpation and cauterization of neoplasms in the nasal cavities and larnyx.

6. The prophylactic method aims to prevent diseases of the respiratory organs by increasing the resistance of the skin and respiratory mucous membranes to the influence of cold, which in many cases is the single cause of the disease (exercise and work, accustoming to cold temperatures, pasturing). With horses, it is especially important to avoid decreasing the resistance of the skin 
by blanketing in the stable. Hot-house breeding has the effect of making the breed too refined (predisposition to tuberculosis and other lung diseases). Finally, isolation and veterinary police control are of great prophylactic importance in dealing with the infectious diseases.

Inhalations in the Treatment of Domestic Animals. - In spite of numerous experimental investigations very divergent views still prevail regarding the therapeutic value of inhalations for man and animals, also concerning the effectiveness of inhalation apparatus and especially on the question as to how far and in what amount inspired medicines penetrate into the respiratory tract. The first scientific investigations on the subject were made in 1858 by Sales-Giron with an inhalation apparatus in which, with the assistance of a compression pump, the medicated fluid was forced under three to four atmospheres pressure through a very fine outlet against a solid plate and thus divided into a very fine spray. In experiments with this apparatus, PietraSanda and Briau did not succed in introducing the inhaled medicine beyond the larynx and therefore concluded that when it was used the sprayed fluid would not generally arrive in the deeper parts of the lungs. New apparatuses were constructed by Siegle, Bergson, Mathieu, Bulling and Wassmuth in which the fluids were sprayed by compressed air or were vaporized by heat. Experiments with these apparatuses did not give uniform results, as will be seen from the following:

Sänger (Münch. med. Woch., 1901) denies the possibility of sprayed fluids penetrating to the deeper parts of the respiratory tract. Upon the basis of experiments with the Siegle apparatus on phantom lungs, he does not believe that any of the fine droplets of sprayed fluids reach the bronchi or pass beyond the larynx when given by inhalation with this apparatus. Consequently, he regards the inhalation of fluids in fine spray as of no therapeutic value. Seige (Dissertation, 1905), on the other hand, concluded from physical investigations with the Bergson-Siegle apparatus that the inhalation-stream can penetrate to the deeper parts of the lungs (no experiments were made upon animals). Emmerich (Münch. med. Woch., 1901) tested the apparatuses of Bulling and Wassmuth on dogs with inhalations of boric acid solution and brine and found the boric acid in the deeper parts of the respiratory tract (margins of the lungs) after the dogs were destroyed; he is of the opinion that nothing reaches the alveoli when the ordinary inhalation apparatus is used and that the medicament reaches these parts only when it is administered in the form of a fine spray with a specially constructed apparatus. After inhalations of gentian violet solution with Bulling's apparatus, von Schrötter (Münch. med. Woch., 1903) found the pigment in the smallest broncli and in the alveoli; he regards the newer apparatuses very favorably. 
Pöschel (Dissertation, 1905) made a very extensive investigation of the subject of inhalation, using different apparatuses (ordinary spray apparatus, apparatuses of Schenk and Bulling) on dogs, rabbits, chickens and pigeons, and reached the following conclusions: Vapors and gases penetrate to the deepest sections of the respiratory apparatus. When powders are inhaled through the nose the greater part is precipitated in the nasal cavities; when inhaled through the mouth they reach the deepest parts of the respiratory apparatus. Fluids in fine spray, when given by inhalation through the nose with Bulling's or with the ordinary apparatus, do not enter the nasal cavities but are precipitated in the nostrils; when inhaled through the mouth with the ordinary apparatus they penetrate to the pharynx and larynx and the upper fourth of the trachea, while with Bulling's apparatus they reach the lower third of the trachea. Consequently, the inhalation of fluids in fine spray is indicated only in diseases of the cesophagus, larynx and trachea. The investigations of Freund (Berl. tierärztl. Woch., 1907) on horses and dogs with the inhalation apparatuses of Siegle, Bulling and Wassmuth have also furnished proof that when coarsely sprayed solutions are inhaled through the nose they are precipitated just within the nostrils, while fluids in fine spray reach only the pharyngeal cavity and at times the upper part of the trachea. In dogs, fluids in fine spray, when inhaled through the mouth, penetrate to the large and small bronchi but never to the alveoli. Kaestle (Zeitschr. für physik. u. diätet. Therapie, 1907-1908) attributes the conflicting views to differences in the arrangement of the experiments. With the new apparatuses, he has succeeded at every attempt in introducing finely sprayed fluids into respiring lungs down to the bronchi and probably also into the alveoli.

\section{EXPECTORANTS}

Synonyms: Promoters of expectoration, chest remedies, mucus-dissolving remedies, cough remedies, pneumatics, bechics, sternutatorics, errhines, ptarmics.

Definition.-The general term expectorants is applied to a group of drugs which possess the property of promoting the expec- . toration of disease products from the respiratory apparatus (expectore). These products are usually mucus, masses of catarrhal purulent and croupous exudates, croupous membranes, pus, blood, dead lung tissue, foreign bodies and parasites, which may be located in the lungs, bronchi, trachea or larynx. More rarely, snuff or other substances that cause sneezing (sternutatorics, errhines) are used to empty the nasal cavities. 
Action.-To understand the action of expectorants, it must be remembered that the body is provided with certain protective arrangements by which it is possible for foreign bodies entering the air passages to be removed without the assistance of medicines. These physiological expectorants are cough, the outwarddirected movement of the cilia of the epithelial cells and, sometimes, sneezing. As a result of catarrhal swelling of the mucous membrane or the deposit of thick, viscid mucus and masses of exudate, the activity of the ciliated epithelium is reduced or suppressed. The same effect is produced when the cilia are involved by excessive catarrhal desquamation, inflammatory infiltration or ulceration, and also when they are paralyzed by the products resulting from the decomposition of the retained bronchial contents (fatty acids). Cough, also an important natural healing force, is sometimes suppressed or abolished by general bodily weakness or by paralytic conditions of the sensory nerves of the mucous membrane (necrosis of the lungs, severe laryngitis). Cough and the movement of the cilia can both be artificially stimulated by certain expectorant drugs. These, however, are not the only methods of action of expectorants; there are several others. In general, the methods of action of the expectorants are as follows:

1. Stimulation of the ciliated epithelium. The principal remedies are ammonium chloride and other ammoniacal preparations: ammonium carbonate, ammonia water and aromatic spirit of ammonia. Most of the stimulant expectorants also increase the activity of the cilia.

2. Stimulant (cough-producing) expectorants. These stimulate either the peripheral sensory nerves of the mucous membrane or the respiratory centre. They include tar, creosote, turpentine oil, camphor, benzoin, balsam of Peru, emetine, ipecac, senega and quillaja. Turpentine oil at the same time decreases the secretions of the respiratory mucous membrane and suppresses the abnormal secretion of mucus.

3. Increase of the gland secretions dissolves mucus and thereby assists expectoration. Gland secretions are increased by 
the following drugs: apomorphine, [terpin hydrate], pilocarpine, arecoline, the antimony salts, sulphur, the alkalies and numerous aromatics.

4. Liquefaction of mucus, chemically or mechanically, is brought about by all of the alkalies and ammoniacal preparations; also by sweet and mucilaginous substances and water (inhalation).

5. The following act as antiseptics or as antiparasitics in putrid decomposition of the contents of the bronchi and bronchiectases, and when bacteria or parasites are present (tuberculous bronchitis, canine distemper, strangles, strongylus, syngamus trachealis): creolin, creosote, tar and turpentine oil.

6. A narcotic effect (allaying cough, bechics) is exerted upon the irritated respiratory mucous membrane by morphine, codeine, heroin, hydrocyanic acid, potassium bromide and other narcotics; also by mucilaginous and sweet substances.

7. The emetics operate as expectorants in a purely mechanical way (see p. 35).

Uses.-The expectorants are the most important drugs in the treatment of the different diseases of the respiratory apparatus. They are prescribed in bronchitis, tracheitis, laryngitis, catarrhal and croupous pneumonia, bronchiectases, cavities in the lungs, pulmonary gangrene, croupous laryngitis, tracheitis and bronchitis, mycotic and verminous pneumonia and all infectious bronchial and pulmonary diseases; also to remove blood, pus, dust and other foreign bodies from the air passages. The errhines (sternutatorics) are used in diseases of the nasal cavity, especially in œstrus larvæ disease of sheep. In dry catarrhs, and also when viscid, sticky, inflammatory products are present (dry râles), the gland stimulants and the mucus-liquefying expectorants are generally prescribed, while the stimulant (cough-producing) expectorants and ciliary stimulants are employed when large collections of fluid disease products are present in the air passages (moist râles).

Drugs. - 1. Ammonii chloridum. Ammonium chloride is used as an expectorant in the later stages of bronchial catarrh, catarrhal and croupous pneumonia, canine distemper, strangles, etc.; stimulates the movement of the cilia, liquefies the mucus 
and increases the secretion of the glands. Dose for cattle, 10-25, 3ijss to vj; horses, 8-15, 3 ij to 3 ss; sheep, goats and swine, 1-2, grs. $\mathrm{xv}$ to $\mathrm{xxx}$; dogs, $0.2-1$, grs. iij to $\mathrm{xv}$; cats, $0.1-0.3$, grs. iss to iv; fowl, $0.05-0.2$, grs. $3 / 4$ to $\mathrm{iij}$.

2. Apomorphinæ hydrochloridum. Apomorphine is a superior expectorant for the liquefaction of viscid masses of mucus in bronchial catarrh, catarrhal pneumonia, canine distemper, etc. Dose internally for dogs, $0.001-0.003$, gr. $1 / 70$ to $1 / 25$ (up to 0.03 , gr. $1 / 3$, per day); horses, $0.02-0.05$, gr. $1 / 4$ to $3 / 4$.

3. Ipecacuanha. Ipecac. A good expectorant for small animals in catarrbal pneumonia, bronchitis and canine distemper. Dose for dogs, $0.01-0.05$, gr. $1 / 8$ to $3 / 4$. Dose of pulvis ipecacuanhæ et opii (Dover's powder), 0.5-2, grs. vij to xxx.

4. Senega and Quillaja. Stimulant expectorants in asthenic bronchitis and catarrhal pneumonia. Dose for dogs in infusion, $0.5-1.5$, grs. vij to xxij; sheep, goats and swine, 1-2, grs. xv to $\mathrm{xxx}$; cats, $0.2-0.5$, grs. ïj to vij.

[5. Terpini hydras. Terpin hydrate. In small doses, increases the secretions of the bronchial mucous membrane; large doses inhibit the secretions. Dose for the dog, 0.1-0.5, grs. jss to vij.]

6. Antimonii et potassii tartras. Tartar emetic. An expectorant for vigorous animals in bronchial catarrh and bronchopneumonia. Doses for cattle, 2-5, $3 \mathrm{ss}$ to $3 \mathrm{i}$; horses, 0.5-2, grs. vij to $\mathrm{xxx}$; sheep, goats and swine, $0.1-0.2$, grs. iss to iij; dogs, $0.01-0.05$, gr. $1 / 8$ to $3 / 4$; cats, $0.005-0.01$, gr. $3 / 40$ to $1 / 8$. [Dose of vinum antimonii for dogs, 5 to 10 drops; cats, one-half.]

7. *Antimonium sulphuratum, Kermes mineral. *Antimonii sulphidum, black sulphide of antimony. Expectorants in bronchitis, canine distemper and strangles. Doses for horses and cattle, 10-25, 3 ijss to vj; sheep and swine, 1-2, grs. $\mathrm{xv}$ to $\mathrm{xxx}$; dogs, $0.05-0.5$, grs. $3 / 4$ to vij.

8. Sulphur lotum, sulphur præcipitatum, sulphur sublimatum. Sulphur. An old expectorant in chronic catarrh. Dose for horses, $2-5,3$ ss to $\mathrm{i}$.

9. Sodii chloridum, sodii bicarbonas, sodii sulphas, *sal Caro- 
linum factitium N.F. (artificial Carlsbad salts). Mucus-dissolving alkalies; used in bronchial catarrhs and in catarrhal and croupous pneumonia. Dose for cattle, $50-100$, 5 iss to iij; horses, $25-50$, 3vj to 3 iss; sheep and goats, $2-5,3$ ss to $i$; dogs, $1-2$, grs. $\mathrm{xv}$ to $\mathrm{xxx}$.

10. Anisum, fœniculum, carum, *juniperus and other aromatics. Good expectorants in mild bronchitis. Dose for cattle, 25-50, 3 vj to 5 iss; horses, $10-25$, 3 ijss to vj.

11. Oleum terebinthinæ, pix liquida, creosotum, *creolin, balsamum peruvianum, benzoinum, camphora and terebinthina. Stimulant expectorants, and at the same time disinfectant, in chronic bronchitis, bronchiectasis and pulmonary gangrene. Dose for horses and cattle, 10-25, 3 ijss to vj; dogs, $0.1-1$, grs. iss to $\mathrm{xv}$.

12. Glycyrrhiza and extractum glycyrrhizæ, saccharum and syrupus. Mild stimulant and solvent expectorants. Dose, ad libitum.

. 13. Morphinæ sulphas. Morphine sulphate. A specific cough remedy for dogs. Dose, $0.01-0.05$, gr. $1 / 8$ to $3 / 4$; usually combined with hydrocyanic acid in the form of aqua amygdalæ amaræ. Dose of the latter for dogs, 0.5-3, nvij to xlv. Codeinæ phosphas or codeinæ sulphas is still better than morphine. Dose, three times that of morphine. 


\section{GENERAL THERAPEUTICS OF THE DISEASES OF THE URINARY ORGANS}

Pathology.-Among the diseases of the urinary organs, those of the kidneys are rather rare in the domestic animals, which is entirely contrary to the relation of these diseases to man. Excluding pyelonephritis, which occurs frequently in cattle but is scarcely accessible to treatment, the only condition affecting the kidneys of practical importance in veterinary therapeutics is nephritis, parenchymatous and interstitial. The latter especially, - which develops into contracted kidneys, is at times the object of treatment in horses and dogs. Diseases of the bladder are much more frequent in the domestic animals. In dogs, simple catarrh of the bladder is most commonly met with, while purulent and hemorrhagic cystitis are the forms of inflammation usually presented in horses and cattle. Cystic calculi and paralysis of the bladder occur frequently in all species. On the other hand, tumors occur only in isolated cases.

Physiology. - The function of the kidneys is the secretion of urine. Different parts of this work are performed by certain parts of the kidneys. The water and the inorganic salts are secreted by the glomeruli; the proper constituents of the urine, especially the urea and uric acid, are secreted by the epithelial cells of the convoluted tubules. The action of the latter cells is an active, specific secretion, while in the glomeruli a purely mechanical filtration process is associated with the action of the epithelial cells. In addition to the water of the urine, bacteria and blood also pass through the walls of the glomeruli in healthy and diseased kidneys. The quantity of urine excreted increases with the rise of blood-pressure and decreases with the fall of the same. Direct or reflex stimulation of the splanchnic causes contraction of the renal blood-vessels and increase of blood-pressure, and a consequent increase of the secretion of urine. The reaction of the urine of herbivora is alkaline (calcium carbonate); of carnivora, acid (phos- 
phates). The bladder is provided with a double set of muscles (sphincter vesicæ, detrusor urinæ); the motor centre for the bladder is in the lumbar cord.

Therapeutic Methods.-Different methods can be used in the treatment of diseases of the kidneys.

1. Acting upon the secreting epithelium may be regarded as the direct method. It consists in the stimulation of the depressed urinary secretion and is essentially medical. See the diuretics (caffeine, theobromine, theocine, diuretin, agurin).

2. Acting upon the renal circulation (blood-vessels, blood, blood-pressure, nervous system) has also the effect, but indirectly, of increasing the secretion or filtration of urine (digitalis, water, milk, alkalies, reflex cutaneous irritation).

3. The derivative method relieves the diseased kidneys by increasing the secretions of the intestinal (cathartics), cutaneous (diaphoretics, baths) and salivary (arecoline, pilocarpine) glands.

4. A disinfectant action can probably be exerted upon the kidneys and also upon the bladder by the use of salicylic acid, tannic acid, uva ursi, etc. This is denied by some.

5. Abnormality in reaction can be removed by the administration of alkalies and acids.

6. Rest of the diseased renal tissues is obtained by bodily quiet and a certain diet (food poor in albumin and producing little urea; milk especially).

7. The operative method includes the removal of cystic calculi and local treatment of the bladder; also the excision of diseased kidneys.

\section{DIURETICS}

Synonyms: Hydragogues, litholytics, lithothryptics.

Actions. - The normal secretion of urine in the kidneys (physiological diuresis) occurs as a result of the coopperation of various factors, of which the most important are the secretory activity of the renal epithelium, the blood-pressure, the blood content of the glomeruli and the diffusion activity of the renal parenchyma. 
In normal conditions, all these factors are operating at nearly their full functional capacity and for this reason, when diuretics are administered to healthy animals, the secretion of urine is either not increased at all, or only slightly or temporarily. On the other hand, in diseases in which there is decreased activity of the gland cells, low blood-pressure, poor blood supply or blood stasis in the kidneys, or depression of the diffusion activity of the renal tissues, the depressed secretion of urine may be increased by numerous drugs. The diureties operate in different ways, corresponding to the complicated processes which are concerned in physiological diuresis. According to their mode of action, the diuretics may be divided into the following groups:

1. Specific stimulants for the secreting renal epithelium: caffeine, theobromine, theocine, diuretin, agurin, calomel, salicylic acid, milk sugar and alkalies. The action of these diuretics is independent of cardiac activity; they increase the secretion of urine even when the blood-pressure is considerably depressed.

2. Circulatory diuretics: digitalis and strophanthus. In consequence of the increase of blood-pressure in the glomeruli of the kidneys produced by these drugs there occurs, in a purely physical manner, an increased filtration of blood-serum through the walls of the glomeruli into the excretory uriniferous tubules. This group of diuretics is most effective in diseases of the heart accompanied by low blood-pressure.

3. Stimulant diuretics (acrics) produce an active hyperæmia, and in consequence an increased secretion of urine. This group includes alcohol and a series of ethereal oils, resins and balsams: turpentine oil, turpentine, juniper berries, oil of juniper, balsam of Peru, balsam of copaiba, petroselinum (parsley), ononis, ligusticum, mustard, black pepper, capsicum, colchicum, cantharides and alcohol.

4. Saline diuretics accelerate diffusion in the kidneys, increase the water content of the blood and, at the same time, mildly stimulate the renal epithelium. They include: sodium chloride, sodium and potassium bicarbonate, sodium and potassium nitrate, sodium and potassium acetate, potassium chlorate, 
potassium tartrate and bitartrate, sodium salicylate and lithium carbonate.

5. Mechanical diuretics, which promote the secretion of urine by flushing out the renal tubules, are: water, milk, and also the organic acids, acetic, citric, tartaric, etc., which by their oxidation increase the water content of the blood.

Uses.-The diuretics are employed therapeutically for many different purposes, the principal of which are the following:

$\checkmark 1$. The resorption of fluid collections in the body in general dropsy, ascites, hydrothorax, hydropericardium, anasarca, œdemas and hydræmia; also fluid inflammatory exudates occurring in the course of pleuritis, peritonitis, serous polyarthritis, pneumonia and acute and chronic hydrocephalus. The diuretics employed in these diseases, which bring about a decrease of the fluids of the body, especially the blood, and thus cause a reabsorption of fluid transudates and exudates, were formerly called hydragogue diuretics.

2. Flushing the renal tubules, the pelvis of the kidneys and the bladder in nephritis, pyelitis and cystitis. In the course of parenchymatous (catarrhal) nephritis, oliguria or even anuria may result from the obstruction of the straight and convoluted efferent renal tubules with urinary casts, desquamated epithelium, fibrinous exudate or blood. These conditions may be overcome by washing the obstructions out of the kidneys. Collections of pus and masses of exudate in the pelvis of the kidneysand in the bladder can be removed in the same manner. Irritant diuretics, however, are to be avoided.

3. The derivation of blood to the kidneys when distant organs are hyperæmic or inflamed (brain, lungs, pleura, intestines, liver).

4. Solution of urinary sediments in the pelves of the kidneys and in the bladder. For this purpose, the alkalies are used. They neutralize the uric acid and form urates of sodium, potassium, calcium and lithium, which are soluble in water.

5. The removal of bacteria, toxins, poisons and metabolic products (uric acid in gout) from the body.

6. To relieve the heart in cardiac diseases. By increasing the 
elimination of water from the blood, the diuretics reduce the volume of the latter and thus diminish the work of the heart.

Drugs.-1. Caffeina'. A specific diuretic in all dropsical conditions; to be preferred to digitalis in dropsies not of cardiac origin (cirrhosis of the liver, contracted kidneys, hydræmia), in which digitalis is ineffective. Dose of *caffeinæ sodio-salicylas N.F., subcutaneously, for horses and cattle, $5-10$, $3 i$ to ijss; large dogs, $0.5-2$, grs. vij to $x x x$; small dogs, grs. iss to vij. It has no cumulative effect; can therefore be given for a long time. *Theobromine and *theocine have the same action.

2. *Diuretin. A very good diuretic; a combination of theobromine with sodium salicylate. Because of the close relationship of theobromine to caffeine, it has the same action and uses as caffeine. Large dogs may be given up to $5, \mathbf{3} i$, in one day. *Agurin, a combination of theobromine with sodium acetate, has the same action.

3. Hydrargyri chloridum mite. Calomel. A good diuretic in cardiac dropsy; to be preferred to digitalis in many cases. Dase for dogs of medium size, $\mathbf{0 . 1}$, grs. iss, three times daily.

4. Acidum salicylicum, sodii salicylas. Salicylic acid, sodium salicylate. Diuretic, especially in muscular and articular rheumatism; also probably disinfectant for the kidneys, renal pelvis and bladder. Dose for horses and cattle, 5-10, $3 \mathrm{i}$ to ijss, two to five times daily; dogs, $0.1-0.5$, grs. iss to vij (small doses).

5. Digitalis. The oldest and a very valuable diuretic in cardiac dropsy. Dose for horses and cattle, 2-5, 3ss to i; dogs, 0.1-0.3, grs. iss to iv. Scilla has the same action.

6. Strophanthus. A powerful diuretic in cardiac dropsy; acts more promptly than digitalis. Dose of tinctura strophanthi for horses, 10-25, 3 ijss to vj; dogs, 10-25 drops.

7. Oleum terebinthinæ. Turpentine oil. A strong and cheap diuretic for dropsy of all kinds. Dose for horses and cattle, 10-25, 3 $\mathrm{ijss}$ to $\mathrm{vj}$; sheep and goats, $2-5$, 3 ss to $\mathrm{j}$; dogs, $0.2-0.5$, wiij to vij.

8. *Juniperus. Juniper. A good, mild diuretic in mechanical as well as inflammatory dropsies. Dose for cattle, 50-100, 5 iij; horses, $25-50,3$ vj to xij; sheep, goats and swine, 2-5, 3ss to j. Dose of oleum juniperi, same as oil of turpentine. 
9. Balsamum peruvianum. Balsam of Peru. A good diuretic and a disinfectant for the kidneys and bladder. Dose for horses and cattle, 10-25, 3 ijss to vj; sheep, goats and swine, 1-2, grs. $\mathrm{xv}$ to $\mathrm{xxx}$; dogs, $0.1-1$, grs. iss to $\mathrm{xv}$.

10. Diuretic species. Diuretic teas of *ononis, *ligusticum and *juniperus; used in dropsies in dogs and cats. Infusions of 25 parts of the drug in 300 of water are given in tablespoonful doses.

11. Sinapis alba, sinapis nigra. Mustard. Mild diuretics. Dose for cattle, 50-100, 争 iss to iij; horses, 20-50, $3 \mathrm{v}$ to xij; dogs, $0.2-2$, grs. iij to $\mathrm{xxx}$.

12. Capsicum, Piper, Cubeba. Stimulant diureties; use cautiously; contraindicated in inflammation of the kidneys. Dose for cattle, 10-25, 3 ijss to vj; horses, $5-15,3 \mathrm{i}$ to iv; swine, 1-2, grs. $\mathrm{xv}$ to $\mathrm{xxx}$; dogs, $0.2-0.5$, grs. iij to vij.

13. Potassii nitras. Potassium nitrate. A good saline diuretic in kidney and bladder affections, also in general dropsy. Dose for cattle, 10-25, 3 ijss to vj; horses, 8-15, 3 ij to iv; swine, 1-2, grs. $\mathrm{xv}$ to $\mathrm{xxx}$; dogs, $0.2-0.5$, grs. iij to vij.

14. Potassii chloras. Potassium chlorate. A disinfectant diuretic in catarrh of the bladder. Dose for horses and cattle, $5-10,3 i$ to ijss; sheep and goats, $1-2$, grs. $x v$ to $x x x$; dogs, $0.1-1$, grs. iss to $\mathrm{xv}$.

15. Potassii acetas, sodii acetas. Potassium acetate, sodium acetate. Uric acid solvents. [Dose for horses and cattle, 15-30 J ss to i; dogs, 0.3 to 1.3 , grs. $v$ to $x x$.]

16. Sodii chloridum, sodii bicarbonas, potassii bitartras. Sodium chloride, sodium bicarbonate, potassium bitartrate. Mild diuretics, which render the urine alkaline and neutralize acidity. Dose for horses and cattle, 25-100, $3 \mathrm{vj}$ to 5 iij; sheep, goats and swine, 2-5, 3ss to j; dogs, $0.5-2$, grs. vij to xxx.

17. Sodii boras. Borax. A mild diuretic, also disinfectant to the bladder and kidneys. Dose for horses and cattle, 10-25, 3 ijss to vj; dogs, 1-2, grs. $x v$ to $x x x$.

18. Saccharum lactis. Sugar of milk. A modern diuretic in cardiac dropsy. Dose for dogs, 25-100, 3 vj to $5 \mathrm{jij}$, daily. Milk diet. 


\section{GENERAL THERAPEUTICS OF THE DISEASES OF THE GENITAL ORGANS}

General Considerations.-Of the diseases of the genital organs, the most important are the affections of the uterus and udder. The diseased conditions to which the uterus is liable, especially before, during and after parturition (dystocias, parturient paresis, puerperal septicæmia, catarrh of the uterus), are so numerous and so widely different that it is difficult to outline general principles for their treatment. This is also true of the diverse conditions which affect the udder. There are, however, several groups of medicines which are usually given special consideration in the general therapeutics of the diseases of the genital organs. These are the ecbolics (uterines), the aphrodisiacs, and the galactagogues.

With regard to the physiology of the uterus, it is to be observed in connection with the action of the ecbolics that the important nerve centre of the uterus (principal centre, centre of parturition) is situated in the lumbar cord at the first and second lumbar vertebræ. The uterus may be stimulated directly and indirectly, centrally and peripherally, by mechanical, chemical, thermic and electrical stimuli. Mechanical stimuli include pressure and blows from without, introduction of foreign bodies into the uterus (the hand, sounds, instruments, injected fluids, air), escape of the amniotic fluid, death of the fœtus, specific uterine catarrh in infectious abortion, hyperæmia and anæmia. Chemical stimuli are the specific uterine drugs (ergot, hydrastis, gossypium and amenyl), and an excess of carbon dioxide in the blood. The thermic stimuli are cold (e.g., reflexly from the stomach) and heat (fever, hot vaginal injections). Contractions of the uterus may also be produced by electrical stimulation of the centres, the sacral plexus and the ischiatic nerve.

Concerning the milk secretion, it is to be noted that the gland cells actively participate in the formation of the specific milk constituents and that the process is not exclusively one of transudation, desquamation of epithelial cells and fatty metamorphosis. The casein and the milk sugar are formed entirely in the gland 118 
cells; the fat is in part derived from the blood. The milk secretion is under the influence of secretory and vasomotor nerves. The quantity and composition are influenced by numerous other factors, as feed, breed, age, lactation period, time of day, work, and exercise and individuality. On this account, the chemical composition is very variable. The average water content is 87.5 per cent.; the average per cent. of dry matter is 12.5 , including 3.3 per cent. fat, 4 per cent. protein, 4.5 per cent. sugar and 0.7 per cent. salts. The reaction is amphoteric, due to the presence of alkaline and acid salts. Curdling of milk may result from different causes (rennet ferment, lactic acid ferment, acids, mastitis).

\section{ECBOLICS. ABORTIVES}

Synonyms: Uterines, ectrotics, amblotics, pellentics, odinegogues, parturefacients, emmenagogues, abortive medicines, expelling, fœtus-expelling medicines.

Actions and Uses.-Drugs exerting a specific action upon the uterus produce either a contraction or hyperæmia of that organ. The result of the action differs with the condition of the uterus. If the uterus is in the act of contracting upon the fœtus to expel iti.e., if the period of pregnancy has terminated and labor has begun, -then the ecbolics increase the force of the contractions and hasten the expulsion of the fœtus. This is the only purpose for which they are of value in veterinary therapeutics. They are therefore administered not before but (1) during parturition, after the beginning of labor pains, to stimulate insufficient uterine contractions in difficult parturition, also in relaxation of the muscles of the uterus, and (2) after parturition, to produce contraction of the relaxed puerperal uterus and thereby cause the more prompt expulsion of the fotal membranes.

The ecbolics will also cause abortion when the full term of pregnancy is not completed. The abortion results either from the contraction of the muscles of the uterus, or from pronounced hyperæmia of the uterus with hemorrhage into the fœtal membranes and into the mucous membrane of the uterus. But the abortive action of drugs is always accompanied by other effects 
dangerous to life and for this reason they are no longer used therapeutically to bring about abortion. Instead, mechanical methods are employed exclusively to produce an artificial abortion or premature birth: forcible dilation of the cervix, puncture of the fotal membranes, insertion of an elastic bougie between the uterus and the membranes, injections between the uterus and fotus, hot uterine douches, tamponading the vagina, etc.

The ecbolics also act upon the empty uterus, causing contraction of the uterus in metritis and checking uterine hemorrhage. Neoplasms of the uterus (myomas) are favorably influenced. Promotion of menstruation by the so-called emmenagogues is only of value in human practice in the treatment of the different menstruation anomalies of women.

Drugs.-1. Ergota. Ergot. An excellent ecbolic in difficult parturition and in retention of the fotal membranes; also a styptic in uterine hemorrhage, and a specific against myomas of the uterus. Dose for cattle, 25-50, 3 vj to xij; horses, 10-25, 3ijss to vj; sheep, goats and swine, $2-5,3$ ss to $\mathrm{j}$; dogs, $0.5-2$, grs. vij to $\mathrm{xxx}$; cats, $0.2-1$, grs. iij to xv; fowl (retention of eggs), 0.2-0.5, grs. iij to vij.

2. Hydrastis. An ecbolic for weak labor and uterine hemorrhages. [Dose of fluidextractum hydrastis for horses and cows, 8-30, 3 ij to $5 \mathrm{i}$; sheep and swine, 4-8, $3 \mathrm{i}$ to $\mathrm{ij}$; dogs, $0.3-4$, 叹 v to $3 \mathrm{i}$.]

\section{DRUGS THAT STIMULATE THE SEXUAL IMPULSE APHRODISIACS}

\section{Synonym: Erotics.}

Actions and Uses.-Artificial stimulation of the sexual impulse is required in phlegmatic, fat or diseased males and females when the sexual desire is below normal. Relaxation and weakening of the body as a result of irrational feeding or stabling, previously existing disease, overexertion, breeding too young, breeding too frequently, masturbation, and the different diseases of the genital apparatus are the usual causes.

The action of aphrodisiacs is exerted in different ways. Liberal feeding of foods rich in nitrogen and the iron preparations operate in a purely dietetic way; fat and phlegmatic animals, on the con- 
trary, require the withdrawal of food, cathartics and metabolic stimulants. The specific erotics produce a hyperæmia, blood infiltration, and increased turgescence, and, in consequence, a highly stimulated condition of the ovaries and testicles and the vaginal or urethral mucous membranes. Yohimbin, cantharides, the acrid spices, alcohol, santonin, the ethereal oils, resins and balsams operate in this way.

Drugs. - Yohimbin. A specific against breeding-impotence in bulls and stallions and decreased sexual impulse and sterility in cows and mares. Only chemically pure preparations should be used, the so-called veterinary preparations being avoided; very expensive ( $1 \frac{1}{2}$ grains, 75 cents). Dose per os for horses and cattle, 0.05 , gr. $3 / 4$; dogs, $0.001-0.002$, gr. $1 / 70$ to $1 / 40$; subcutaneously for horses and cattle, $0.02-0.05$, gr. $1 / 4$ to $3 / 4$; sheep, goats and swine, $0.005-0.01$, gr. $3 / 40$ to $1 / 8$; dogs and chickens, $0.0005-$ 0.001 , gr. $1 / 140$ to $1 / 70$; canary birds, 0.0001 , gr. $1 / 660$.

2. Cantharis. Cantharides. An aphrodisiac for cows in which œestrum is abnormally weak; administer cautiously. Dose for cows, $2-5,3$ ss to $i$, per day; horses, $0.5-2$, grs. vij to $x x x$. Dose of tinctura cantharidis for cows, $20,3 \mathrm{v}$; horses, 10 , 3 ijss; dogs, drop doses.

3. Piper, Capsicum, Cubeba, Zingiber Santonica, etc. Dos for cattle, $10-25$, 3 ijss to $\mathrm{vj}$; horses, $5-15,3$ i to iij.

4. Alcohol. In small repeated doses; horses and cattle, 20-50, $3 \mathrm{v}$ to $x i j,(20-150,3 \mathrm{v}$ to $3 \mathrm{v}$, spiritus vini gallici; $1 / 2$ to 1 liter, Oi to $\mathrm{ij}$, vinum album).

5. Santoninum. Santonin. Dose for horses and cattle, 10-25, 3 ijss to $\mathrm{vj}$.

*Juniperus, carum, matricaria, aloe and other drugs are also used as erotics, but their action is uncertain.

\section{DRUGS THAT DEPRESS THE SEXUAL IMPULSE ANAPHRODISIACS}

Synonyms: Antaphrodisiacs, anterotics.

Actions and Uses.-An excessive sexual impulse (nymphomania, satyriasis) occurs most frequently in cows (bulling) in consequence of pathological changes in the genital organs, espe- 
cially the ovaries and uterus. In stallions, the sexual desire is increased in cryptorchids and masturbators. The action of the anterotics consists of a soothing effect upon the nerves of the sexual organs. All sedative neurotics are therefore anaphrodisiacs, particularly potassium bromide, chloral hydrate, and morphine. A restricted diet and cathartics may also be prescribed in addition. A certain and permanent removal of abnormal sexual impulse can be accomplished as a rule only by operative methods (castration, cryptorchid operation, ovariotomy, rectal compression of ovarian cysts).

Drugs.-1. Potassii bromidum. Potassium bromide. A very good anaphrodisiac, especially for nymphomania in cows. Dose for horses and cattle, 20-50 (up to 150 per day), $3 \mathrm{v}$ to xij (up to $5 \vee$ per day); dogs, $0.25-2$ (up to 10 per day), grs. iv to $x x x$ (up to 3 ijss per day); cats, $0.25-0.5$ (up to 2 per day), grs. iv to vij (up to grs. $\mathrm{xxx}$ per day).

2. Chloralum hydratum. Chloral hydrate. Uses and doses same as potassium bromide.

3. Morphinæ sulphas. Morphine sulphate is not always reliable, especially for cattle; recommended in combination with chloral hydrate. Dose, subcutaneously, for horses, 0.5, grs. vij; dogs, $0.02-0.1$, grs. $1 / 4$ to iss.

Mild laxatives, bleeding, hunger and exercise also have anterotic effects.

\section{DRUGS THAT INCREASE MILK SECRETION GALACTAGOGUES}

Synonyms: Lactics, galactics, stimulants to milk secretion.

Actions and Uses.-The so-called milk defects, which are due to abnormal feeding, gastro-intestinal catarrh and other diseased conditions, udder diseases and various external influences, are of especial practical consideration in cattle practice. Those best known are agalactia (absence of milk), watery milk, excessively fat milk, premature curdling, failure of milk to "butter," putrid, soapy and slimy (viscid) milk, blue, red and yellow milk.

The latter defects are due to external infection and are therefore to be combated with antiseptics. With reference to the 
action of the specific galactagogues, a distinction must be made between those which act upon the general condition and those which act directly upon the udder. Drugs belonging to the first category are called indirect galactagogues and those of the second direct galactagogues. Since the greater number of the milk defects result from digestive disturbances and general diseases, an improvement in the milk secretion can be brought about indirectly by treating these diseases. The indirect galactagogues include particularly the aromatics, bitters and alkalies, which by their gastric action promote digestion and in consequence increase the formation of the milk constituents. Cutaneous stimulation, directly applied to the udder, stimulates the gland secretion reflexly, and is therefore also an indirect galactagogue. It is well known that frequent milking increases the milk production by stimulating the secretory nerves of the gland. This is also true of electric stimulation, as has been demonstrated by faradization of the udder. Rubbing the udder with stinging nettle, as is done in many countries (goat herds in Verona), has the same effect. On the other hand, the antimony preparations and sulphur are direct galactagogues, as might be expected from their stimulant action upon the other glands of the body. The effect of these drugs in increasing the milk secretion is probably the result of direct action upon the secreting cells of the udder. To what extent other galactagogues stimulate milk secretion by increasing the blood-pressure (see the experiment by Röhrig below) or by acting upon the secretory nerves of the gland has not at this time been determined with certainty. But it is probable that with the secretion of milk, as with the similar secretory processes of diuresis and diaphoresis, it must be assumed that medicines operate in several ways to increase the activity of the udder.

Experimental Investigations of the Actions of the Galactagogues.-The knowledge concerning the action of medicines upon the milk secretion, quantitatively and qualitatively, is far less complete than that relating to the other secretions, e.g., diuresis. In the first place, a distinction must be made between the popular, empirical views regarding the action of certain drugs upon the secre- 
tion of milk and the conclusions based upon exact pharmacological experiments. In veterinary medicine, the following drugs have been for a long time regarded as galactagogues: the different aromatics, particularly fennel, anise, caraway, juniper berries, coriander, dill, fine-leaved water hemlock, pimpinella, calamus, the bitter stomachics (yarrow and gentian), sulphur, the neutral salts, the antimony preparations (sulphurated antimony, antimony), and others. These drugs are the chief constituents of the popular milk powders. In human medicine, the leaves of polygala vulgaris, galega officinalis and the castor-bean plant are recognized as galactagogues, while iodine, belladonna, quinine and alum, together with external applications of opodeldoc, are chiefly used as anti-galactagogues. According to Niebuhr, in many sections of Germany oil of rosemary, a constituent of opodeldoc, is rubbed on the udder of cows to reduce the milk secretion with good results. Applications of oil of hemp have a similar effect. The results of experiments with the individual galactagogues and anti-galactagogues are very contradictory. The most important of these investigations are the following:

Experiments to test the power of iodine to reduce the milk secretion were made by Labourdette (Gazette des Hôpitaux, 1856). He found that after the administration of potassium iodide to cows, goats and asses, the milk secretion was at first somewhat increased (1/10 to $1 / 8)$, then rapidly decreased, and finally in the course of 2 to 3 months, under the continued action of potassium iodide, ceased entirely, the experimental animal becoming very much emaciated. When daily doses of 3 to 6 grams of potassium iodide were given, the milk contained up to $1 / 4$ gram per liter, and the drug could be demonstrated in the milk 12 days after the administration of the last dose. A slight, primary increase in the milk secretion after the administration of potassium iodide was also observed by Lewald. Bouley (Bulletin de l'Académie, 1859) reported a long series of experiments with potassium iodide in which the milk was not changed quantitatively and was otherwise in good condition, but after curdling it had a rubber-like elasticity and plasticity. Stumpf demonstrated that 5 grams of potassium 
iodide daily caused a considerable decrease of the milk secretion in goats in consequence of a disturbance of the gland function. Alcohol (up to 100 c.c.) and alcoholic drinks (1-2 liters of beer) produced no change in the quantity of milk secreted by goats, but increased the relative fat content. Morphine, pilocarpine and lead salts had no effect on the quantity or quality of the milk. Salicylic acid appeared to increase somewhat the quantity of milk and the sugar content. Röhrig, in his experiments on goats (Virchow's Archiv., Bd. 57), observed no alteration of the milk secretion even after very large doses of morphine (up to 1.2). On the other hand, he reports a considerable acceleration in the milk secretion after the administration of jaborandi leaves (pilocarpine), a result which is disputed by other experimenters (Partsch, Heidenhain) who obtained negative results in similar experiments. After the administration of drugs which increase blood-pressure (strychnine, caffeine, digitalis), Röhrig observed an increase in the quantity of milk, while the amount was decreased when drugs were given which reduce blood-pressure (potassium bromide, chloral hydrate, etc.). According to Feser (Jahresbericht der Münchener Tierarzneischule, 1885, 1889 and 1890), the milksecretion of bealthy cows was slightly increased after the injection of eserine and pilocarpine; a greater increase occurred in his experiments with eseridin. Feeding experiments conducted by him, in which the milk of these cows was fed to swine, demonstrated that milk from cows treated with eserine could be fed to swine without any disadvantage whatever. Hess, Schaffer and Bondzynsky (Landw. Jahrb. der Schweiz, 1888) tested the effect of fennel, calamus, anise, caraway, juniper berries and gentian upon the milk secretion of healthy cows and goats and found that even very large doses of these drugs had no real influence upon the quantity or quality of the milk. The drugs mentioned were not perceptible in the milk through any change of taste or odor. Only in the case of fennel, the odor of the drug was observed in an alcohol-ether extract of the milk. Fröhner's own experiments (Monatshefte für praktische Tierheilkunde, 1891) with sodium bicarbonate, sulphur, sulphurated antimony, juniper berries, atropine and pilocarpine on 
six healthy cows of different breeds, namely, Dutch, Dithmarsch, Simmenthaler, Swiss, Wilstermarsch and Shorthorn, and on two healthy goats, were also entirely negative. Fingerling (Landw. Versuchstat., 1907) found in his experiments with goats that odorous and tasty substances increased the secretion of the milk gland, while others do not; for instance, the addition of oil of fennel or of sodium chloride to mixed feed increases the fat per cent. as well as the quantity of milk, but the addition of arsenic has no effect. According to Kronacher (Berl. Tierärztl. Woch., 1910) the milk secretion is increased by yohimbin.

The explanation of the contradictory results of these experimental investigations is perhaps to be found in the following circumstances. It is to be noted first of all that the experiments of all of the investigators were made on healthy animals. In practice, however, the galactagogues are not administered to healthy but to diseased animals. As is well known, the action of a drug upon a healthy animal is often different from its action on a diseased animal. The failure of a drug to act on healthy animals does not prove it to be ineffective on diseased animals. This very important principle has often been overlooked in therapeutics. The value of the bitters as stomachics, for example, would have to be denied, if this principle is not accepted, because they have an indifferent effect on normal digestion. Empiricism teaches that the bitters possess a superior gastric action only in disturbed digestion, and this is confirmed by clinical experiments. An entirely similar condition exists with regard to the antipyretics, which, as is well known, only reduce an elevated and not the normal body temperature. It should not, therefore, be assumed that the galactagogues are without effectin disturbed lactation because of the negative results of experiments on healthy animals. Furthermore, some observers have actually obtained positive results from the administration of galactagogues to healthy animals.

Drugs.-1. *Antimonii sulphidum. Black sulphide of antimony. A much-used galactagogue in agalactia of cows and goats. Dose for cows, 10-25, 3 ijss to vj; goats, 1-2, grs. xv to xxx.

2. Sulphur lotum, sulphur præcipitatum, sulphur sublimatum. 
Sulphur. Combined with the previous drug in milk powders. Dose for cows, 2-5, 3ss to j; goats, $0.5-1$, grs. vij to xv.

3. Anisum, fœniculum, carum, *juniperus. Empirical galactagogues in different milk defects. Dose for cattle, 25-50, $3 \mathrm{vj}$ to xii; goats, $2-5,3$ ss to $\mathrm{j}$.

4. Sodii chloridum, sodii bicarbonas. Sodium chloride, sodium bicarbonate. Galactagogues in all milk defects, the latter especially in premature curdling; usually combined with those previously mentioned. Dose for cattle, $50-100$, 5 iss to iij; goats, $5-10,3 \mathrm{i}$ to $\mathrm{jjss}$.

\section{DRUGS THAT DEPRESS MILK SECRETION ANTIGALACTAGOGUES}

Synonyms: Antigalactics, lactifuges, alactics.

Actions and Uses.-An excessive production of milk occurs in some animals, especially non-pregnant bitches in œstrum, which at times requires therapeutic interference. A cessation of the milk flow may be desired in cows for purely economical reasons, as when the animal is to be fattened for the butcher. Artificial reduction of the milk secretion also becomes necessary in mares and bitches when lactation persists after the death or removal of their offspring. As antigalactagogues, laxatives, potassium iodide, tannin and alum may be tried, in addition to reducing the feed and gradually increasing the intervals between milking and finally stopping. Local applications of oil of rosemary or oil of hemp to the udder are also recommended for cattle. The action in the first case is an inhibitory effect upon the secretion and in the latter probably a derivative effect (see p. 124).

Drug.-Potassii iodidum. Dose for horses and cattle, 5-15, 3 i to iv; dogs, $0.25-0.5$, grs. iv to vij. 


\section{GENERAL THERAPEUTICS OF THE GLANDS (DIA- PHORETICS AND SIALAGOGUES)}

Physiology.-The glands, especially the sweat, salivary and mucous glands, are, as secretory organs, of great importance in therapeutics because their physiological activity can be artificially increased for curative purposes. This physiological activity consists in the secretion of water, and also of specific and pathological constituents, which are eliminated from the body with the gland secretions. The secretion is the result of an active function of the gland cells, which is under the influence of specific secretory nerves, and is not a mere filtration. For this reason drugs acting specifically upon the glands (arecoline, pilocarpine) are required to artificially stimulate the gland secretion, while drugs which only increase the blood-pressure (digitalis) and thereby promote filtration are without effect. The gland secretions are indirectly influenced, however, by the quantity and quality of the blood and its rate of flow, and also by the dilation and contraction of the bloodvessels (vasomotor nervous system).

The sweat glands are especially well developed in the horse. The general sweat centre is situated in the medulla oblongata, and there are also special centres for the anterior extremity in the end of the cervical cord and for the posterior extremity in the lumbar cord. The secretory fibres pass from the centres to the sweat glands by way of the motor nerves. The secretion of sweat can be increased by direct stimulation of the centres (increase of the body temperature, fever, diaphoretics) or by reflex stimulation (cutaneous irritation, various thermic and electrical stimuli, increased muscular activity). The most important constituents of normal sweat in addition to water ( 97.5 to 99.5 per cent.) are urea, volatile fatty acids (formic, acetic, butyric, caproic and caprylic acids) and salts (sodium chloride, potassium chloride, sodium and potassium phosphate, sodium and potassium sulphate and calcium, magnesium and iron compounds). The average amount of sweat secreted 
daily by horses at work is 10 to 20 liters; this amount can be increased three-fold with arecoline or pilocarpine. The salivary glands (parotid, submaxillary, sublingual, glands of the cheeks, lips and gums) are connected with nerve centres in the medulla oblongata and brain. Their peripheral secretory nerves are the facial, sympathetic and glossopharyngeal; vasomotor and motor nerves are also present. The secretion of saliva is increased directly by the stimulation of the salivary centres and nerves (arecoline, pilocarpine) and reflexly by the ingestion of food, by mechanical, chemical, thermic and electrical stimulation of the mucous membrane of the mouth and stomach; also by stimulation of the splanchnic nerves. The constituents of saliva, in addition to water (98 to 99.5 per cent.), are ptyalin, albumin, mucin and salts (sodium chloride, sodium carbonate, phosphates, sulphates, potassium sulphocyanide). The normal salivary secretion of the horse amounts to 10 to 40 liters; by artificial stimulation with arecoline and pilocarpine this quantity can be obtained in a few hours (laminitis).

\section{DRUGS THAT STIMULATE THE SECRETION OF SWEAT DIAPHORETICS}

Synonyms: Hidrotics, sudorifics, diapnoics.

Actions.-The drugs which stimulate the secretion of sweat cause an increased evaporation (diapnoics) or an increase in the amount of visible, watery sweat secreted by stimulation of either the peripheral nerves of the sweat glands, the sweat centres in the medulla or the secretory cells of the glands; by depression of the inhibitory nerves, or by dilation and relaxation of the cutaneous vessels and consequent hyperæmia of the skin. Those which operate upon the nerves or cells of the sweat glands (hidrotics) may be designated as direct diaphoretics. To this group belong arecoline, pilocarpine and ammonium acetate. Indirect diaphoretics, which first produce hyperæmia of the skin and then increased diaphoresis (sudorifics), include warm water internally and moist heat externally. Finally, sweating is also stimulated reflexly by emetics. 
The effects of increased secretion of sweat upon the organism are very numerous. The most important is the loss of water from the body. This causes, like diuresis and catharsis, a resorption of fluids contained in the tissues or body cavities. At the same time there is a derivation of the blood from the internal organs to the skin, with increased dissipation of heat from the latter and a consequent fall of the internal temperature (critical sweat of febrile diseases). The secretory activity of the kidneys is relieved, the excretion of urea and other metabolic products through the skin is increased and the body weight is reduced, while the activity of the heart is lessened because of the decrease in the volume of the blood. Moreover, bacteria and their toxins, grape sugar, bile pigments, drugs and poisons (mercury, lead, iodine, bromide) are eliminated from the blood through the sweat.

Uses.-Since the introduction of pilocarpine and arecoline, the diaphoretics are of greater therapeutic importance in veterinary medicine, especially in equine practice. The most important diseases in which they are indicated are the following:

(a) Dropsical conditions in the course of heart, lung, liver and kidney diseases, particularly hydrocephalus, ascites, hydrothorax, and hydropericardium, pleuritic and peritoneal exudates, and exudative inflammation of the articulations.

(b) Laminitis and articular and muscular rheumatism.

v(c) Diseases of the kidneys, especially oliguria, anuria and uræmia occurring in the course of severe inflammation of the kidneys.

$\checkmark$ (d) Chronic poisonings (mercury, lead).

(e) Tetanus, cerebrospinal meningitis, parturient apoplexy.

Drugs.-1. Pilocarpine. In large doses, a powerful diaphoretic; specific in laminitis in horses, also in muscular rheumatism; superior resorbent for all dropsies, transudates and exudates, particularly in acute hydrocephalus of the horse. Dose of pilocarpinæ hydrochloridum (subcutaneously) for horses is 0.5-0.8 (!), grs. vij to xii (!). The hidrotic action will be promoted by rubbing and warm, moist (vinegar) coverings. A rather expensive drug. 
2. *Arecoline. Also a powerful diaphoretic and a specific against laminitis; to be preferred to pilocarpine because less expensive (compare the dose) and more stable. Dose of *arecolinæ hydrobromidum for the horse (subcutaneously), 0.05-0.1, grs. $3 / 4$ to iss.

3. Hot infusions of the aromatics: matricaria, *flores tiliæ, *sambuci flores, mentha piperita, and *melissa. These 'diaphoretics of the old therapeutics are still sometimes employed, e.g., in rheumatic colic of the horse. Their diaphoretic action is due principally to the warm water which the infusions contain. They are still very commonly used in human medicine.

4. Washing the skin with vinegar and then applying a Priessnitz dressing. A very good method of assisting the action of pilocarpine and arecoline.

5. *Ammonii acetas is sometimes prescribed internally as a diaphoretic. Dose for the horse, 10-25, 3ijss to vj. [Liquor ammonii acetatis, spirit of mindererus, contains about 7 per cent. of ammonium acetate. Dose for the horse, 60-120, 年ij to iv; dogs, $8-30,3$ ij to viij.]

\section{DRUGS THAT STIMULATE THE SECRETION OF SALIVA SIALAGOGUES}

Synonyms: Sialics, ptyalogogues, masticatives, masticatorics.

Actions and Uses.-Of the drugs known at this time, only pilocarpine and arecoline really possess a pronounced specific effect upon the salivary glands. These drugs stimulate the peripheral nerves of the salivary glands and the salivary centre and thereby increase the secretion of saliva to a great degree and for some time, even when given in small doses. Eserine and muscarine are similar in action but much weaker.

The effects resulting from an increased secretion of saliva are very similar to those produced by diaphoresis. Of special importance in therapeutics is the circumstance that a large amount of water is withdrawn from the body, the blood therefore becoming thicker and the resorption of transportable fluids in the body being thereby promoted. A horse, for example, will lose 20 to 30 liters 
of water in a short time. Sialagogues are consequently specific for all dropsies, transudates and fluid exudates. They have an abortive action against laminitis (arecoline), act as derivatives in diseases of the brain and kidneys and serve to eliminate poisons from the body.

Antisialagogues are drugs which reduce an abnormally increased secretion of saliva (salivation). The most important are atropine and hyoscine (scopolamine). The same drugs also at the same time reduce an abnormally increased sweat secretion and are therefore called antihidrotics. To the latter class belong also agaricin and camphoric acid.

Drugs.-1. Pilocarpinæ hydrochloridum. Specific sialagogue in dropsical conditions. Dose ( $1 / 5$ the diaphoretic dose): horses, $0.1-0.2$, grs. iss to iij; cattle, $0.2-0.4$, grs. iij to vj; sheep and goats, 0.01, gr. $1 / 8$; dogs, $0.005-0.02$, gr. $3 / 40$ to $1 / 4$. Use cautiously when heart disease is present.

2. *Arecolinæ hydrobromidum. Also a specific sialagogue; especially effective in laminitis and acute hydrocephalus. The dose of arecoline is five to ten times less than that of pilocarpine; for horses, $0.02-0.05$, grs. $1 / 4$ to $3 / 4$. 


\section{GENERAL THERAPEUTICS OF THE DISEASES OF METABOLISM}

Physiology.-The treatment of the so-called metabolic diseases (diabetes mellitus, obesity, gout, chronic disturbances of nutrition) must be based upon the physiology of metabolism. Metabolism can be influenced principally in a dietetic way. The chief nutritive materials used for this purpose are albumin, fat and the carbohydrates, in addition to water and salts.

In reference to albumin, physiology teaches that the more albumin that is introduced into the body the more is decomposed. Feeding albumin in abundance therefore only increases the total albumin decomposition and does not increase the amount stored up. A real strengthening of the body, i.e., an increase in the amount of organic albumin, does not result from a pure albumin diet, contrary to the usual assumption. Such a diet only temporarily increases the amount of circulating albumin in the blood. If organic albumin is to be stored up in the body, which is the object aimed at in the treatment of chronic disturbances of nutrition, then fats or carbohydrates must be given with the albumin. The presence of fats and carbohydrates limits the albumin decomposition in both healthy and diseased animal bodies.

The fat taken into the body is partly stored up and partly decomposed. The fat deposited in the body is derived principally from the fat in the food and only to a slight degree from the albumin. In emaciation, therefore, the deposition of fat can be attained only by feeding an increased amount of fat and not by increasing the albumin in the diet. On the contrary, reduction can be obtained in obesity by an exclusively albuminous diet (Banting treatment) or by withdrawing fat and carbohydrates from the ration. 
The carbohydrates serve in the first place as heat-producing material for the body (exercise). Increased ingestion of carbohydrates decreases the decomposition of albumin and fat. They are consequently an important dietetic material in the treatment of chronic disturbances of nutrition accompanied by emaciation (with the exception of obesity and diabetes mellitus, in which the carbohydrates should be withheld as completely as possible).

A stimulating influence upon metabolism is exerted by muscular activity and exercise (increased oxidation of carbohydrates and fats, not the albumins), cutaneous irritation, salts (sodium chloride, sodium bicarbonate, sodium sulphate), alcohol, and arsenic and phosphorus in small doses. See also therapeutics of the glands.

In the general therapeutics of the diseases of metabolism, the remedies are usually divided into plastics, antiplastics (removal of fat) and nutrients.

\section{PLASTIC REMEDIES. PIASTICS}

Synonyms: Nutrients, euplastics, antatrophics, antidepertitorics; foods, blood plastics, bone plastics.

Definition and Classification.-Plastics are substances which participate directly or indirectly in the construction and building up of the animal body. They can be subdivided into blood plastics (see p. 63), bone plastics, muscle plastics, etc., corresponding to the individual organs. They can also be classified as foods and medicinal plastics.

(a) The foods (dietetic remedies in the restricted sense) are naturally the most important plastics. In therapeutics, less consideration is given to the food as such than to an effort to supply the usual food in the most readily digestible form (sick diet). Horses affected with certain diseases, for instance, are given oats which have been ground or crushed, or are fed steamed oats; carnivora are given meat finely ground; young animals are fed eggs, leguminous meals containing dextrin (children's flour); mucilaginous gruels, roasted meal, etc., are fed in intestinal catarrh. See the text-books on dietetics. 
(b) The medicinal plastics correspond essentially to the foods in composition and consequently consist of albumin, fat, carbohydrates and salts. The most important are meat extract, cod liver oil, sugar, starch flour, milk sugar, peptone, iron salts, calcium salts, phosphorus, phosphates, sulphur, potassium salts, sodium chloride and other salts. A former special subdivision of the medicinal plastics was the peptic plastics, which included pepsin and hydrochloric acid. These are referred to under physiological stomachics (page 29). Arsenic is an indirect plastic.

Uses.-The plastics are employed to improve nutrition in all acute or chronic diseases which are accompanied by emaciation or tissue reduction and especially by anæmia. These changes are most frequently observed in chronic constitutional diseases, particularly anæmia, pernicious anæmia, leukæmia, rachitis, osteomalacia and diabetes mellitus. They also occur in many chronic debilitating diseases, e.g., tuberculosis, worm cachexia, chronic gastric and intestinal catarrh and carcinomatosis. Considerable emaciation takes place in all acute febrile diseases in which metabolism is greatly increased, especially in the acute infectious diseases (contagious pneumonia, canine distemper, aphthous fever).

Plastics are contraindicated in obesity, which on the contrary is to be combated with antiplastics. (See the section on antiplastics, p. 138.)

Drugs.-1. *Meat extract. A valuable blood plastic in anæmia and leukæmia in consequence of its content of blood salts (potassium phosphate, iron); also a general plastic in chronic disturbance of nutrition in carnivora. It has no particular nutritive value because it does not contain albumin, carbohydrates or fats. Dose for dogs, 2-10, grs. xxx to 3 ijss.

2. Oleum morrhuæ. Cod liver oil. On account of its content of free fatty acids and easily digested fat, an important plastic in rachitis and anæmia and following exhausting diseases. Doses for horses and cattle, 100-500, 5 iij to Oi; sheep, goats and swine, $50-100,3$ iss to iij; dogs, $10-50,3$ ijss to 5 iss; fowl, $2-5$, m xxx to $3 \mathrm{i}$.

3. *Peptone. A substitute for albumin; plastic in chronic disturbances of nutrition in carnivora. Dose for dogs, 10-25, 3 ijss 
to $\mathrm{vj}$; cats, $5-10,3 \mathrm{i}$ to ijss. Other nutrient preparations are: somatose, nutrose, nutrol, mutase, eucasin, roborin, eulactol, sanose, sanatogen, aleuronat, alkarnose, protogen, tropon, globon, etc.

4. Ferrum. Iron. A specific in anæmia and leukæmia; administered in small frequently-repeated doses in the form of ferrum reductum, ferri sulphas, tinctura ferri chloridi and other iron preparations (see p. 63). The blood coloring matter itself is also used in the form of *hæmoglobin, *hæmatol, *hæmatogen, *trefusia, damholid, etc.

5. Calcii phosphas præcipitatus. Precipitated calcium phosphate. A specific against rachitis and osteomalacia; usually employed in the form of the so-called bone precipitate. Dose for cattle, 25-50, 3 vj to xij; horses, 10-25, 3 ijss to vj; calves, foals, sheep, swine and goats, $5-15,3 \mathrm{i}$ to $\mathrm{jv}$; dogs, 0.5 to 5,3 ss to $\mathrm{j}$; fowl, $1-2$, grs. $x v$ to $x x x$. Calcii carbonas præcipitatus and liquor calcis are also used.

6. Phosphorus. A specific against rachitis and osteomalacia; also a plastic and a stimulant to the bone tissue. Usually combined with cod liver oil. Dose for horses and cattle, $0.01-0.05$, gr. $1 / 8$ to $3 / 4$; sheep and swine, $0.002-0.005$, gr. $1 / 40$ to $3 / 40$; dogs, $0.0005-0.002$, gr..1/140 to $1 / 40$; cats and fowl, $0.0005-0.001$, gr. $1 / 140$ to $1 / 70$.

7. Sulphur præcipitatum, sulphur lotum, sulphur sublimatum. A general plastic (sulphur content of the tissue albumins). Dose for horses and cattle, $2-5$, 3 ss to $j$; dogs, $0.05-0.2$, grs. $3 / 4$ to iij.

8. Potassii bicarbonas. Potassium bicarbonate. A blood plastic (blood-corpuscles). Dose for cattle, 50-100, 䒓iss to iij; horses, 25-50, 3vj to xij; sheep, goats and swine, 2-5, 3 ss to ij; dogs, $0.5-2$, grs. vij to $x x x$.

9. Sodii chloridum. Sodium chloride. The plastic most used in animal husbandry; a muscle, blood and gland plastic (bloodplasma). Dose, same as potassium bicarbonate.

10. *Sal Carolinum factitium N.F. Artificial Carlsbad salts. In small doses (same as sodium chloride), increases metabolism and is in consequence a good plastic. 


\section{NUTRIENTS. ROBORANTS}

Synonyms: Tonics, confortatives, confortants; invigorating, strengthening medicines.

Definition and Uses.-The nutrients differ from the foods in that they are not constituents of the animal body, but they correspond to the foods in action in that they relieve disturbances of nutrition and metabolism and strengthen the body. Some of them act as substitutes for the proper food and therefore serve to economize tissue waste. They form a group of drugs which stand between the plastics and excitants. The former designation of tonics is incorrect because the nutrients do not increase the tension of the tissues, as the muscles for instance, but stimulate metabolism and the specific functions of the cells, and act upon the muscles in the same manner as upon the nerves and glands.

The roborants of importance in veterinary medicine include alcohol, which on the one hand in consequence of its oxidation to carbonic acid and water saves the carbohydrates and fats of the tissues, and on the other hand stimulates the functions and the metabolism of the cells of the body, especially those of the brain, heart, skeleton muscles and glands. Wine, beer, brandy, cognac and other fluids containing alcohol act in the same manner as alcohol. Other very important roborants are coffee and other substances containing caffeine (tea, cocoa). Arsenic is also very frequently used as a roborant.

The diseased conditions in which the nutrients are used are the disturbances of nutrition and conditions of weakness which occur in the course of chronic and acute diseases and after difficult parturition and severe hemorrhage.

Drugs.-1. Alcohol. The best roborant in the weak conditions occurring in the course of febrile infectious diseases (contagious pneumonia); in convalescence following severe acute or chronic diseases; in cachectic conditions, and after difficult parturition, hemorrhages, etc. At the same time, it economizes tissue waste and acts as an excitant. Given in repeated small doses: 
horses and cattle, 25-50, $3 \mathrm{vj}$ to xij; dogs, 2-5, 3 ss to i. Dose of spiritus vini gallici double these quantities. Wine (vinum album, vinum rubrum) and beer are administered in quart (horses and cattle) and tablespoonful (dogs) doses.

2. *Caffea. Coffee. A superior excitant for weakness and depression of the brain, spinal cord, heart and the muscles of the body.

3. Arseni trioxidum. Arsenic. A roborant in chronic nutritive disturbances and in conditions of inanition, especially in general emaciation, anæmia, rachitis, osteomalacia and chronic eczema. Dose for horses and cattle, 0.1 to 0.5 , grs. iss to vij; sheep and swine, $0.01-0.05$, gr. $1 / 8$ to $3 / 4$; dogs, $0.001-0.005$, gr. $1 / 70$ to $3 / 40$ ( 2 to 10 drops of liquor potassii arsenitis).

\section{REDUCING REMEDIES. ANTIPLASTICS}

Synonyms: Dysplastics; reducing treatment, abstinence treatment, hunger cure.

Actions.-The antiplastics are used exclusively to combat and overcome obesity or corpulence, i.e., an abnormal collection of fat in the subcutaneous connective tissue, liver, heart, body cavities, etc., resulting from too intensive feeding with insufficient exercise or work. The antiplastics are usually divided into two groups: the dietetic and medicinal.

Dietetic Reducing Methods.-These consist of methodical, rational feeding and regulation of the diet, i.e., the supplying of suitable foods and the partial or complete withdrawal of certain nutritive substances and foods. The simplest reducing method, which usually suffices for animals, is the hunger or abstinence cure. This method is applied by entirely withholding food from the over-fat animal for a time or by reducing the amount of food given. Work and exercise at the same time will be of assistance in obtaining the desired results. This method, however, is not applicable to all cases. In advanced obesity, in which the body is deficient in albumin, the abstinence cure will increase the weakness. In other cases, the method cannot always be carried out (ill-behaving 
dogs). For these reasons, the modern dietetic reducing methods used on man possess a certain importance in veterinary medicine, especially in canine practice. The most important of these methods are the following:

(a) The Banting ${ }^{1}$ cure. This consists of the use of a purely albuminous diet, the only food permitted being fat-free meat. Carbohydrates and fat are omitted entirely. As a consequence, in order to meet the carbon requirements of the body, the fat stored up in the tissues is drawn on and oxidized. Great care must be exercised in using this method. If continued too long, until after the tissue fat is used up, the tissue albumin will be attacked and will be reduced in spite of the increased ingestion of albumin.

(b) The Ebstein ${ }^{2}$ reducing method. In this method only the carbohydrates are excluded from the food, the use of fat as well as albumin being permitted. The fat is given to bring about an early appeasement of the appetite and thus decrease the total amount of food ingested.

(c) The Oertel ${ }^{3}$ reducing method. This method is based essentially upon the withholding of water; albumin, fat and carbohydrates are all permitted. In addition, an effort is made to increase the amount of work performed. This method is especially adapted to the treatment of fatty degeneration of the heart. In consequence of the withdrawal of water, the blood is decreased in volume and the resistance to the heart is thereby reduced. The simultaneous artificial increase of cardiac activity exerts a fatreducing and strengthening effect upon the heart muscle.

Medical Reducing Remedies.-These are secondary in importance to the dietetic methods. They involve the use of cathartics, especially Glauber's salt. The frequently repeated rapid emptying

${ }^{1}$ W. Banting, an Englishman suffering from obesity, was treated by his physician, Dr. Harvey, by the method formerly known as the "meat method," and published his experiences in 1863 in a book entitled "Letter on Corpulence Addressed to the Public."

2 Ebstein, late professor of medicine in Göttingen.

- Oertel, late professor of medicine in Munich. 
of the intestinal contents removes from the body a quantity of nutritive materials and the excess of body fat must finally serve as a substitute. At the same time, through the specific action of the alkalies, the metabolism of the body is accelerated and the oxidation of the fat is thereby increased. Most frequently, Glauber's salt is used in the form of Carlsbad salts. The treatment of obesity with potassium iodide (chronic iodism) and with preparations of the thyroid gland (iodothyrin) is dangerous. 


\section{GENERAL THERAPEUTICS OF DISEASES OF THE EYE}

Pathology.-In the treatment of the different diseases of the eye numerous therapeutic methods come into consideration. Diseases of the lids, conjunctiva and cornea are treated with antiseptics (boric acid, salicylic acid, chlorine water, quinine), astringents (zinc sulphate, silver nitrate), caustics (copper sulphate, oxide of mercury, cauterization) and narcotics (cocaine, eucaine, alypin, anæsthesin, orthoform); also by operation (entropion operation), massage (corneal opacity), hydrotherapy, etc. In paralysis of the optic nerve, excitants (strychnine) and electrotherapy are employed. Finally, there is a group of medicines which are usually designated as eye remedies in the restricted sense. These are the pupil-dilating (mydriatics) and the pupilcontracting (myotics) agents.

\section{PUPIL-DILATING REMEDIES. MYDRIATICS}

Actions and Uses.-The artificial dilation of the pupil by atropine, scopolamine (hyoscine) and homatropine is the result of paralysis of the oculomotor nerve the motor nerve of the sphincter of the pupil. The mydriasis is usually produced by the direct or local action of the drug by introducing the latter into the conjunctival sac; dilation of the pupil by the internal administration of the mydriatic is of no practical value.

The purposes for which mydriatics are used are very variable. Therapeutically, they are employed in iritis to prevent or loosen adhesions of the iris to the capsule of the lens. For this reason, they are of the greatest importance in the treatment of periodic ophthalmia in the horse. They are also used to prepare the eye for some operations, especially the cataract operation and iridectomy. They are not less important for diagnostic purposes, when it is desired to examine the interior of the eye, the artificial dilation of the pupil facilitating the examination of the interior with the ophthalmoscope. In this way turbidity of the vitreous humor 
and of the lens, detachment of the retina and other internal eye diseases are more readily recognized.

Drugs.-1. Atropinæ sulphas. The most frequently used mydriatic in periodic ophthalmia, iritis and in operations on the eye; 1 to 2 per cent. solution. For diagnostic purposes a weaker solution is used ( $1 / 2$ to 1 per cent.).

2. Hyoscinæ hydrobromidum. A more powerful mydriatic; therefore used when atropine does not suffice; solution, 0.2 to 0.5 per cent.

3. Homatropinæ hydrobromidum. A mydriatic used for diagnostic purposes. The pupillary dilation disappears more rapidly than when produced by atropine or hyoscine, but occurs much more slowly. Very expensive.

Newer mydriatics are *mydrol, *mydrin, *eumydrin and * euphthalmine (very expensive).

\section{PUPIL-CONTRACTING REMEDIES. MYOTICS}

Actions and Uses.-The myotics, of which arecoline and pilocarpine may be mentioned as the most important, cause a narrowing of the pupil by contraction of the sphincter muscle of the pupil, the latter action resulting in consequence of stimulation of the oculomotor nerve. Myosis is of value therapeutically because by the contraction of the iris synechia of the latter with the capsule of the lens will be loosened. Adhesions of the iris occurring in periodic ophthalmia, for instance, are treated alternately with atropine (mydriasis) and eserine (myosis). Eserine is also employed to reduce the internal pressure of the eye in glaucoma.

Drugs.-1. Physostigminæ sulphas. Eserine. A myotic in synechia of the iris, periodic ophthalmia, adhesion of the iris and prolapse of the iris; $1 / 2$ to 1 per cent. solution.

2. *Arecolinæ hydrobromidum. Used in the same manner as physostigmine. 


\section{GENERAL THERAPEUTICS OF THE SKIN AND MUCOUS MEMBRANES}

Physiology.-The skin and mucous membranes are in the first place protective organs, guarding the body against external influences. The skin especially being exposed to numerous and varied insults must possess a resistant structure and a particularly strong regenerative capacity. This is actually the case, as is demonstrated most markedly in the healing of wounds. (See the chapter of general surgery on primary and secondary healing of wounds.) No loss of tissue is more readily replaced by natural healing than epithelium or epidermis. In many cases, natural healing alone removes the defect.

In addition to their protective action, the skin and mucous membranes have other important physiological functions. These latter include secretion (water, mucus, urea, salts, bacteria, toxins, gases), respiration (cutaneous respiration), regulation of heat (evaporation, radiation) and reflex action. The reflex activity of the skin and mucous membranes is of the greatest importance in therapeutics because of the numerous specialized nerveend apparatuses they contain and also because of the manifold relations of the skin to the internal organs (central nervous system, circulatory apparatus, respiration, kidneys, gastro-intestinal canal, metabolism). (See the chapter on the so-called cutaneous irritant or derivative method.)

Therapeutic Methods.-The many different diseases to which these structures are liable, together with the extensive relations of the skin to the internal organs, combine to make the therapeutics of the skin and mucous membranes a very comprehensive subject. Wounds, inflammation, eczemas, catarrhs, infections, parasites, tumors and many other disease conditions call for numerous special methods of treatment. Of these, the antiseptic (including disinfection) treatment of wounds, the antiparasitic method of treatment and hydrotherapy, as well as the diaphoretic method of 
treating internal diseases, must be discussed in special chapters. The operative treatment of tumors and wounds must also be omitted from this section. For the prophylactic method (hardening of the skin to cold, care of the skin, clipping), the reader is referred to the text-books on hygiene and dietetics. There remain to be considered of the general therapeutic methods the cutaneous irritant method, the caustics, firing and the astringent method. In addition, the treatment of the diseases of the skin will be reviewed in a general way.

\section{GENERAL THERAPEUTICS OF THE DISEASES OF THE SKIN}

Eczema.-The very diverse and essential differences in the individual diseases of the skin render a discussion of the subject in a general way very difficult. Leaving out of consideration those skin diseases belonging to the domain of surgery (dermatitis, tumors, ulcers, phlegmona, erysipelas, foreign bodies, gangrene, acne and furunculosis) and the acute infectious exanthemas (pox, aphthous fever, swine erysipelas), there still remains of the skin diseases in a restricted sense a group of dermatoses known under the name of eczema which are of great practical importance. In special pathology, a distinction is made between parasitic and non-parasitic eczema. For the treatment of parasitic eczema (mange, herpes, lice, fleas) the antiparasitic method is employed. This is described in detail in another place (p. 160). The nonparasitic eczema appears, as is well known, in many different forms. There are recognized erythematous, papular, vesicular, pustular, crustaceous, squamous, seborrhœic, sycosiform, acute and chronic eczema. The treatment of the several forms is very different. Next to the causal method, which consists in removing and combating the cause (removal of external irritants, cleaning, disinfection), the symptomatic treatment of the local alterations is the most important. The derivative and internal treatment of eczema with cathartics and arsenic, formerly employed, has been abandoned and replaced by the local treatment. The local treat- 
ment of eczema differs with the form, degree and age of the disease. Chronic eczema especially requires treatment essentially different from the acute. In the one case a stimulant, sometimes even caustic, treatment is necessary (tar in chronic eczema), while in the other protective remedies are indicated (protective and indifferent medicines in acute eczema). Again, other cases require the application of desiccants (silver nitrate, zinc oxide, tannin), emollients (fats, soaps, glycerin), and anodynes (narcotics). In addition, the mechanical remedies (rubbing, massage) and hydrotherapy (baths, poultices) are also employed.

Drugs.-1. Pix liquida. Tar. The best remedy in chronic eczema; contraindicated in acute eczema; used in the form of tar spirits, ${ }^{1}$ tar salve ${ }^{2}$ and tar liniment. ${ }^{3}{ }^{*}$ Creolin acts the same as tar. Modern substitutes for tar are: *ichthyol, *thiol, *tumenol, *thigenol, *lithyol, pyrogallol, *anthrasol, *anthrarobin, *empyroform, *lenigallol and petrosulphol.

2. Zinci oxidum. Zine oxide. A good protective and desiccant in acute eczema; used in powder, ointment [unguentum zinci oxidi], paste and gelatin. Plumbi oxidum, acidum tannicum, *tannoform, bismuthi subgallas, amylum, sevum præparatum (prepared suet), kaolinum and calcii carbonas præcipitatus have the same action.

3. Argenti nitras. Silver nitrate. The best desiccant, protective and disinfectant in acute moist eczemas; used in ointment or in solution in water or alcohol (5 to 10 per cent.).

4. Soaps. Sapo mollis (alkaline) is used as a stimulant in chronic eczema alone or in combination with tar; contraindicated in acute eczema. *Potassa sulphurata (liver of sulphur) has the same action, but is more powerful. The neutral soaps have a cleansing and softening action.

5. Glycerinum. Glycerin. An emollient in squamous and crustaceous eczemas; contraindicated in acute eczemas (stimulant action). Iodine and glycerin combined have a desiccant action.

[ 1 Pix liquida $5 i$, alcohol $5 x$.

${ }^{2}$ Pix liquida $3 i$, adeps $3 x$.

- Pix liquida, sapo mollis, aa 5 iv; alcohol $5 i j$. 
6. Fats, oils, paraffin ointment, petrolatum, lanolin (adeps lanæ hydrosus). Protective, indifferent substances, replacing the cutaneous fat, in acute eczemas; exert a disintegrating action in chronic skin exudates. Pastes (zinc, starch, petrolatum), glycerin gelatin (zinc gelatin), plasters (lead plaster, salicylic soap plaster) and varnishes are similar in action.

7. Arseni trioxidum. Arsenic. Used internally as an alterative in the form of liquor potassii arsenitis ( 5 to 10 drops for dogs; $5-10,3 \mathrm{i}$ to ijss, for horses). More modern arsenical preparations are salvarsan and atoxyl.

\section{CUTANEOUS IRRITANTS. ACRICS}

Synonyms: Derivants, epispastics, rubefacients, vesicants, pustulants, suppuratives, irritants, erethistics, dermerethistics; acrid, stimulating, irritating, derivative medicines; digestive medicines in a surgical sense; blisters.

Actions. - The term acrics is used to designate those drugs which act as irritants to the skin and mucous membranes and to all organs in general which contain nerves and blood-vessels, producing hyperæsthesia and pain together with hyperæmia and inflammation. In the restricted sense the term includes only the cutaneous irritants. The effects resulting from the action of the acrics correspond exactly to the changes produced in the skin by other causes of inflammation (traumatic, thermic and infectious irritants). Numerous investigations concerning the production of inflammation by acrics have been made since those of Cohnheim, among which the work of Leber is especially worthy of consideration. In the purely aseptic inflammation, produced by cantharides or croton oil and not by bacteria, the following changes are to be observed: First, the vessels are dilated reflexly, producing hyperæmia, redness, swelling and increased temperature in the affected part of the skin or mucous membrane. Then follows an exudation of plasma (serum) from the blood. Soon after this there is an emigration of leucocytes through the cement substance of the endothelium. The principal cause of this emigration is not the amœboid movement of the leucocytes, the increased blood-pressure nor 
the greater penetrability of the vessel walls, but the chemotactic action of the chemical irritants, which attracts the leucocytes, sets up the amœboid movement and causes them to wander out to the chemotactic centre (cantharides, croton oil). The emigrated leucocytes act as phagocytes (Metschnikoff) and also produce a digestive enzyme which transforms the disease products into albumoses (propeptones) and by this action liquefies them (histolysis). The term digestives, formerly applied in surgery to those drugs which stimulated suppuration, was therefore entirely warranted. The bacteria-free pus produced by the digestives possesses a strong bactericidal action. There is also probably an increased local formation of antitoxins in consequence of stimulation of the tissue cells by the acrics. (See the chapter on hydrotherapy.) According to the force of the chemical irritation, different degrees of cutaneous inflammation are produced: hyperæmia, vesicle and pus formation. Another, although smaller, division of cutaneous irritants acts like the caustics by coagulation or solution of the albumin of the tissues. (See the following chapter on caustics.)

The skin and mucous membranes differ very greatly in their sensitiveness to the acrics. The reason for this is to be found in the difference in the covering epithelium. The horny, dry, dead cells of the epiderm protect the skin against the action of dry, solid irritants, so that it is indifferent, for example, to sublimate, silver nitrate, arsenic and even to phosphorus in the dry condition, but when these substances are applied to the tender, moist cells of the mucous membrane, with its covering of fluid, they are immediately dissolved and penetrate the membrane. On the other hand, the skin is more susceptible than the mucous membranes to some of the fluid irritants, especially those which are volatile. Turpentine oil, for instance, is less irritant to the tracheal and intestinal mucous membranes than to the external skin because the mucous membranes are covered with a protective layer of mucus which the ethereal oil, not being miscible with water, cannot penetrate, while it is quickly imbibed by the dry cells of the epiderm and can also rapidly pass through the same in the gaseous form. 
Classification.-The cutaneous irritants are usually divided into the following four subdivisions:

1. Rubefacients: Turpentine oil, camphor, tincture of arnica, tincture of capsicum, oil of juniper, oil of rosemary, ammonia water, tincture of iodine, Priessnitz dressing, massage.

2. Vesicants: Cantharides, oil of mustard.

3. Pustulants: Tartar emetic, croton oil, euphorbium.

4. Suppurants: Biniodide of mercury, bichromate of potassium.

Uses.-The cutaneous irritants are of essentially greater practical consideration in veterinary medicine than in human therapeutics. They are employed with advantage in internal medicine as well as in surgery for the most varied purposes. The most important diseases in which they are used are the following:

1. Subacute and chronic inflammations, including chronic eczema, also contusions of the skin, the subcutis and the underlying tendons, tendon sheaths, joints, muscles and bones. Strong cutaneous irritation, such as may be produced by briskly rubbing in tincture of iodine, cantharides ointment or biniodide of mercury ointment, exerts a healing effect in all these surgical conditions. An artificial inflammation is generated in the skin which extends to the underlying parts, converting the chronic inflammation into the acute form, which runs a more rapid course; accelerating, like firing, the absorption of the exudate and, in painful inflammation of the articulations, hastening the ankylosis and thereby removing the pain and lameness. The method of operation of epispastics has been more exactly studied in connection with tincture of iodine. When the latter substance is painted upon the skin an erysipelatous-like inflammation with œdema formation is produced, which is accompanied by outwandering of the white blood-cells (local hyperleucocytosis), formation of enzymes, liquefaction of the tissue albumins and softening of the solid disease products (histolysis). Resorption of the inflammatory exudate follows.

2. Acute inflammations of the lungs, pleura, and peritoneum. The cutaneous irritants first of all draw blood from the internal organs (derivative action). In exudative pleurisy (contagious 
pneumonia), for instance, briskly rubbing the corresponding chost wall with oil of mustard will produce an intense hyperæmia and swelling of the skin, which results in a corresponding withdrawal of blood from the pleura. In the same manner, the skin may derive blood from other internal organs, as the intestines, brain, kidneys. In addition, the formation of antitoxins will be increased in consequence of the stimulation of the nutrition of the body cells.

3. Fever. The cutaneous irritants operate to reduce fever by drawing a larger amount of blood to the skin and consequently increasing the dissipation of heat, like digitalis. They also increase the formation of antitoxins in the body, thereby promoting the natural healing reaction in febrile diseases. By the application of mustard spirit in contagious pneumonia, for example, not only is a derivative action obtained but the temperature is also considerably reduced. At the same time, the heart-beat is slowed.

4. Diseases due to chilling, especially rheumatic colic. The cutaneous irritants (turpentine oil, spirit of camphor) operate in these cases like the diaphoretics, stimulating the evaporation from the skin and regulating the disturbed distribution of the blood (hyperæmia of the central organs, anæmia of the skin).

5. Relaxation and paralysis of the gastric and intestinal musculature. The peristaltic movements of the stomach and intestines may be stimulated reflexly by irritation of the sensory nerves of the skin. The epispastics are therefore very valuable peristaltics in atony and paresis of the gastro-intestinal wall, particularly in the course of constipation colic, tympanites and chronic gastro-intestinal catarrh.

6. Spinal paralyses (lumbar weakness, Iumbar paralysis). The stronger cutaneous irritants act here partly as stimulants to the paralyzed nervous system and partly as derivatives. For the same reasons, they may also be employed in cerebral and peripheral paralyses. In other cases, according to the principles of counter-irritation, the epispastics will depress a pathologically increased nervous activity (hyperæsthesia).

7. Diseases of the kidneys. Because of the ability of the skin to assume in a compensatory way the functions of the kidneys, 
cutaneous irritants afford relief to the kidneys in anuria and uræmia occurring in the course of inflammation of the kidneys.

8. In surgery, the so-called digestives, particularly turpentine oil, aloes and Peru balsam, are used in the form of solutions, ointments, plasters and tampons to promote the suppuration and granulation of wounds, ulcers and abscesses (bactericidal and solvent action of sterile pus).

Zschokke, in an excellent article (Ueber die Wirkungsweise der Derivantien, Monatshefte für prakt. Tierheilkunde, vol. ix, 1898), strongly recommends the retention of the derivants, i.e., the cutaneous irritants, liniments, blisters and firing, in veterinary medicine. According to him, they are indispensable in numerous diseases. He refers to the widespread view that the cutaneous irritants only act by forcing the patient to keep the diseased part as quiet as possible, and points out that the local effects are stimulation of phagocytosis (resorption, disinfection) and an increased formation of antitoxins locally (disinfection) as a result of stimulation of the nutrition of the tissue cells. The general action of the derivants in febrile diseases is also due to the formation of antitoxins, which combat fever and even abort infectious diseases. In phlegmona especially, an effective disinfection, with phagocytosis and a rapid disappearance of the bacteria, is obtained from "blisters," as has been demonstrated experimentally in horses by Bossi (II nuovo Ercolani, 1891).

An historical study of the action of "blisters" was published by Grammlich in the Zeitschrift für Veterinärkunde (1898). See also Künnemann's Festrede über die Wirkung der hautreizenden Mittel (1907).

Drugs.-1. Oleum terebinthinæ. Turpentine oil is frequently used as a rubefacient in chronic muscular rheumatism, colic, chronic articular and tendon lamenesses, cedematous swellings of the skin, contusions of the joints, weak and paralytic conditions, etc.; usually diluted, $1: 10-20$, with alcohol, spirit of camphor or oil; also injected subcutaneously to produce derivative abscesses (so-called fixation abscesses) in infectious diseases.

2. Spiritus camphoræ. Spirit of camphor. Also a frequently used epispastic, alone or combined with turpentine oil. A stronger action is exerted by the application of camphora in substance.

3. Tinctura arnicæ. Much used by the laity as a stimulant and resolvent in rheumatism and contusions. Tinctura capsici has 
the same action and is sometimes, in addition to the previously mentioned drugs, a constituent of the so-called restitution fluid.

4. Tinctura aloes. An old veterinary digestive for granulating wounds and ulcers of the hoof.

5. Aqua ammoniæ. Ammonia water. Usually used in the form of a liniment, 1 part to 4 of oil. A brisk epispastic in chronic arthritis, inflammation of the tendons and muscular rheumatism.

6. Tinctura iodi. Tincture of iodine. A powerful resolvent, especially in periostitis, arthritis, periarthritis, tendinitis and tendovaginitis. Like ammonia water, it occupies a position between the rubefacients and the vesicants.

7. Cantharis. Cantharides. The ointment is frequently used as a vesicant for horses and cattle in chronic exostoses (spavin, ring bone, splint), inflammations of joints, tendons and tendon sheaths, chronic rheumatic lameness, contusion swellings, etc. Cantharides plaster ${ }^{5}$ and cantharides oil ${ }^{6}$ have the same action, but tinctura cantharidis and collodium cantharadatum are somewhat weaker and are employed in canine practice.

8. Oleum sinapis volatile. Oil, of mustard. A valuable derivant in pleurisy, pneumonia, peritonitis and meningitis; used on horses in 4 to 6 per cent. alcoholic solution; cattle, 10 to 20 per cent.; dogs, 2 per cent. Mustard plasters are also frequently used.

9. Hydrargyri iodidum rubrum. Biniodide of mercury. A most powerful epispastic; specific for long-standing inflammations of the joints, bones and tendons, especially spavins, ring bones, splints, galls and induration of tendons; used on horses in concentrations of $1: 4$ to 5 of lard or cantharides ointment. Potassii dichromas has the same action (1:10 to 20 for horses).

10. Antimonii et potassii tartras. Tartar emetic. A derivant for cattle and swine in ointment $(1: 4)$.

[ 4 Powdered cantharides 2 parts, digested for ten hours with 2 parts each of peanut oil and lard; yellow wax 1 part, turpentine 2 parts, mixed; euphorbium 1 part.]

[ 6 Colophony and turpentine, 6 parts of each, melted together; add, after partial cooling, powdered cantharides 3 parts, powdered euphorbium 1 part.

- Cantharides 3 parts, digested ten hours with 10 parts of peanut oil; express and filter.] 
11. *Euphorbium. A very irritant epispastic; used in old, chronic surgical affections of the joints, tendons and bones; used on cold-blooded horses and cattle either alone $1: 10-15$ of lard, or as an addition to cantharides ointment $(1: 10-20)$.

12. Oleum tiglii. Croton oil. Also a very powerful irritant; used in obstinate chronic rheumatism, arthritis, tendinitis, paresis, etc.; also as a derivant in inflammations of internal organs; must be used cautiously. The concentration for horses is $1: 30$; cattle, $1: 5-10$; swine, $1: 2-5 ; \operatorname{dogs}, 1: 50-100$. It is diluted with olive oil, turpentine oil or cantharides ointment.

\section{CAUSTICS}

Synonyms: Corrosives, cauterics, escharotics, cathæretics.

Actions.-While the cutaneous irritants produce only the alterations of inflammation, without injuring the vitality of the tissues, the caustics cause the death of those living tissues with which they come in contact. The caustic action of the individual drugs is brought about in very different ways and on this basis the following groups of caustics are classified (concerning firing, the actual cautery, see p. 156).

1. Caustic acids: Nitric, hydrochloric, sulphuric, acetic, chloracetic, lactic and salicylic acids and formaldehyde. Of these, the mineral acids and lactic acid operate by coagulating the albumin; they unite with the albumin, forming insoluble acid albuminates, thereby bringing about the precipitation and death of the dissolved living albumins. Nitric acid, especially, forms with the organic albumin a yellow acid albuminate (xanthoproteic acid, nitrogen compound). The organic acids, particularly acetic acid, also unite with the tissue albumin to form acid albuminates, but these compounds are soluble and are only precipitated when the solution is neutralized. The caustic action of sulphuric acid is due to its strong affinity for water, which it withdraws from the tissues, the latter being carbonized. Salicylic acid and formaldehyde are peculiar in their action, producing a keratolytic effect, or cornification. 
2. Caustic alkalies: Caustic potash, caustic soda, caustic lime, Vienna paste. These dissolve the organic albumin by taking up water and forming soluble alkaline albuminates. Their eschar or slough is mostly moist, deep and diffuse, extending into the surrounding tissue, a contrast to the dry, circumscribed, superficial eschar of the mineral acids.

3. Metallic caustics: Zinc chloride, corrosive sublimate, potassium chromate, chromic acid, iron chloride, iron sulphate, copper sulphate, zinc sulphate, silver nitrate, antimony trichloride, arsenic, lead acetate and nitrate, biniodide of mercury, oxide of mercury, copper acetate, potassium permanganate. These metallic compounds act by forming an insoluble metallic albuminate, at the same time setting free the acid components (sulphuric acid, nitric acid, chlorine, iodine). Some of them, in addition, act also by oxidation; these include the oxygen-containing caustics, chromic acid and permanganate of potash, which give off oxygen, and also arsenic, which first takes up oxygen and then gives it off. The halogens, chlorine, bromine, iodine, absorb the hydrogen of the tissues, in consequence of which oxygen in the nascent state (in addition to hydrochloric acid) is set free.

The degree of the caustic action differs with the chemical nature of the individual drugs and also with the concentrations employed. A distinction is made between a superficial scab formation and a deeper cauterization. The inorganic acids, silver nitrate, copper sulphate, zinc sulphate, iron sulphate and lead nitrate act superficially, while formaldehyde, the caustic alkalies, sulphuric acid and chromic acid have a deep action. The caustic effect upon the skin is much weaker than upon the mucous membranes because of the protective layer of horny epidermal cells. The dry caustics, especially the metallic salts, act upon the skin generally only when they come in contact with fluids. The color of the eschar differs very much. Nitric acid produces a yellow eschar; hydrochloric acid, a gray; sulphuric acid, brown or dark gray; chromic acid and formaldehyde, yellow, becoming brown and finally black; acetic acid, white; silver nitrate, at first white, then gray and finally black. 
Uses.-The caustics are used to remove neoplasms when they cannot be removed by operation. In general, their use has diminished very much with the progress of surgical technic, but in veterinary medicine there are still several forms of neoplasms for which caustics are preferred to operation on practical or economical grounds. In canker of the frog especially cauterization with formaldehyde is frequently employed instead of operating because of its greater simplicity and convenience. In addition, caustics are sometimes used in the treatment of fistulæ and ulcers, and also to regulate the healing of wounds, especially to remove exuberant granulations (silver nitrate). The expediency of the earlier method of treating herniæ with caustics may be left in abeyance. On the other hand, caustics are indicated in infected and poisoned wounds, particularly potassium permanganate, iron chloride and chromic acid in snake bites.

Form.- The caustics are used in different forms, partly alone and partly in combination with other substances (caustic pastes, pencils, points, bougies, sounds, cords, ligatures, crystals and powders).

Drugs.-1. Liquor formaldehydi. Formaldehyde. The strongest caustic; very penetrating caustic action (be cautious); best caustic in canker of the frog.

2. *Fuming nitric acid. A frequently employed and valuable liquid caustic in veterinary medicine; used in the treatment of canker of the frog and to remove warts and small neoplasms.

3. Acidum sulphuricum. A very strong caustic; used in the treatment of shoe boils, canker of the frog and other neoplasms; use cautiously.

4. Acidum trichloraceticum. Trichloracetic acid. A good but rather expensive caustic for canker of the frog, warts and granulations.

5. *Vienna paste. A mixture of equal parts of caustic lime [calx] and caustic potash [potassii hydroxidum] with sufficient alcohol to make a paste; very powerful caustic for canker of the frog and carcinoma.

6. Zinci chloridum. A strong caustic; used in ulcers, fistulæ, 
relaxed granulations, canker of the frog and carcinoma in the form of caustic paste or pencils.

7. Hydrargyri chloridum corrosivum. Corrosive sublimate. The strongest metallic caustic; used in the form of crystals, powder, paste $(1: 1-5$ flour), salve $(1: 1-10)$, solution ( $1: 3-10$ alcohol or collodion), bougies or caustic ligature. Hydrargyri iodidum rubrum has the same action.

8. Chromii trioxidum. [Formerly official in U. S. as chromic acid.] A powerful, painful caustic for canker of the frog, carcinoma and other neoplasms in solution, salve, paste $(1: 1-5)$ and bougies; superior alterative action in scratches of long standing (10 per cent. solution in water). A specific in snake bites.

9. Arseni trioxidum. Arsenic. An old caustic in breast boils, shoe boils, canker of the frog, carcinoma, quittor, etc., in the form of sticks, paste, salve and solution.

10. Argenti nitras. Silver nitrate. A mild, superficial caustic (escharotic) for granulations, fistulæ and ulcers, chromic conjunctivitis and very small warts; used in pencil form.

11. Cupri sulphas. Copper sulphate. A caustic in ophthalmology; used for granular and pannus growths on the conjunctiva, also employed in fistulæ and ulcers. *Cuprum aluminatum has the same action and uses (copper pencil).

12. Ferri sulphas. Iron sulphate. A mild caustic for ulcers and fistulæ, canker of the frog, the foot lesions in foot-and-mouth disease and chronic affections of mucous membranes. Used in powder or in concentrated solution.

13. Plumbi acetas. Lead acetate. A mild caustic for canker of the frog. Plumbi nitras has a similar action.

14. Ferri chloridum. Iron chloride. A caustic for canker of the frog and relaxed granulations. A specific against the virus of rabies and snake bites.

15. Hydrargyri oxidum flavum. Yellow oxide of mercury. A mild caustic for wounds and ulcers $(1: 10)$; also used as a caustic in ophthalmology $(1: 15-25)$.

16. Potassii permanganas. Potassium permanganate. A specific caustic for snake bites. 


\section{FIRING. CAUTERIZATION}

General.-Firing is one of the oldest methods of treatment and specifically a veterinary remedy, which cannot be dispensed with even to-day, despite the large number of similarly acting chemical substances (caustics). It is, on the contrary, among the most valuable of surgical aids. As a rule, wrought cautery irons of various forms (knife, hatchet, button, conical form, etc.) are used in firing, in addition to which Paquelin's platinum cautery apparatus is used in clinics and on small animals. There are several varieties of firing: superficial (point and line firing), percutaneous, perforating (spavin firing) and subcutaneous. (See the text-books on operative surgery.)

Actions.- The effects of firing are very numerous:

1. The immediate result is a local destruction of tissue. The heat first coagulates the albumin, then follows the formation of an eschar and carbonization. Associated with this action is the disinfecting and hæmostatic effect of the hot iron.

2. A hyperæmia and inflammatory reaction develops in the region immediately surrounding the cauterized area. The circulation of blood and resorption are stimulated in consequence. An extensive outwandering of white blood-cells, local hyperleucocytosis, phagocytosis and histolysis occurs, similar to that which follows the rubbing in of tincture of iodine (see p. 148).

3 . The pronounced irritation of the cutaneous nerves acts reflexly upon the nervous system. Firing consequently operates like a powerful cutaneous irritant (principle of cutaneous irritation and counter irritation).

4. The eschar which results from firing exerts, according to the usual acceptation, a mechanical pressure upon the underlying tissues and promotes, like massage, the resorption of the pathological products (?).

Uses.-1. The most important indications for the employment of the firing iron are the chronic inflammatory conditions of the bones, joints, tendons and tendon sheaths: spavin, ring bone, external spavin (rehbein), curb, splints, chronic arthritis, tendinitis, tendovaginitis and articular galls. While the firing iron should 
be used only on the chronic inflammatory affections of the tendons and tendon sheaths after treatment with hydropathic dressings, massage and blisters has proven ineffective, it may be employed primarily in the aforementioned bone and deforming articular inflammations, especially spavin, ring bone and splints. The effect of firing on all these conditions consists essentially in the conversion of a chronic inflammation into a more rapidly progressing acute process. The reactive inflammation set up in the affected bone, tendon or articulation brings about a more rapid resorption of the inflammatory products, promotes the inflammatory reproductive processes, and in joints hastens ankylosis, in consequence of which the pain and lameness are removed (spavin, ring bone).

2. The firing iron is used for operative purposes on fistulæ, neoplasms and ulcers; as a hæmostatic, particularly in amputation of the tail, and for the disinfection of infected wounds (rabies, anthrax, glanders, snake bites).

3. It is also employed as an excitant, although more rarely, in the treatment of paralytic conditions of the spinal cord and peripheral nerve branches (parturient apoplexy, spinal and peripheral paralyses).

\section{ASTRINGENTS}

Synonyms: Exsiccants, coagulants, tonics; contracting, drying, tanning, albumin-coagulating, condensing, toning remedies.

Actions and Uses.-The term astringents is used to describe drugs which exert a contracting, drying, condensing action upon the skin and mucous membranes. They are most frequently employed in catarrhs of the mucous membranes, with excessive catarrhal secretion or extensive swelling of the mucous membrane, e.g., in catarrhs of the intestinal, buccal, pharyngeal, bronchial, conjunctival, bladder, uterine, vaginal and preputial mucous membranes, and in prolapses of the vagina, uterus and rectum. They are also used in inflammatory, eczematous and ulcerous processes of the skin; in otorrhœa; as injections in fistulæ and serous sacs; 
to check parenchymatous bleeding, and, empirically, in galactorrhcea, polyuria and nephritis.

In their method of action they are very different. Some cause a contraction and narrowing of the capillaries with consecutive anæmia (metallic salts), thus overcoming inflammatory hyperæmia of the skin and mucous membranes and decreasing the secretions. Others combine with the albumin, mucus, and gelatin-containing substance of the secretions of the skin and mucous membranes and form a solid precipitate and produce, like the caustics, a superficial condensation and desiccation of the tissues, forming a kind of scab or protective covering (tannin). A third group operates by withdrawing water and shrinking (alcohol), and a fourth group purely mechanically through compression (collodion). Most of the astringents possess a disinfectant action and, in the stronger concentrations, are caustics. (See the chapter on caustics.)

Drugs.-1. The metallic astringents: alum, aluminum acetate, ferric chloride solution, iron sulphate, lead acetate and nitrate, silver nitrate, corrosive sublimate, zinc chloride and sulphate, copper sulphate, zinc oxide, bismuth nitrate, subnitrate, subsalicylate and subgallate, aluminum hydroxide, calcium carbonate and lime water.

2. Tannic acid, tannoform and the tannin-containing vegetable astringents: white oak bark, cinchona, rhubarb, nutgall, catechu [replaced by gambir in U. S. P.], rhatany root, tormentilla root, salvia, uva ursi, folia juglandis, coffee and roasted acorns.

3. Alcohol and all alcoholic liquids (tinctura aloes, tinctura myrrhæ, tinctura iodi).

4. Iodine, especially in combination with alcohol (iodine tincture) and glycerin.

5. Tar, creolin, creosote and other related substances.

6. Ethereal oils, balsams and resins (turpentine oil, turpentine, Peru balsam).

7. Glycerin and sodium chloride (withdrawal of water).

8. Collodion (mechanical compression) and other pressure agents. 


\section{Ergot, hydrastis, cocaine and adrenalin. (The latter con-} tracts the vessels of the conjunctiva and iris.)

\section{Cold in the form of ice or cold applications.}

Antiphlogistics.-A series of medicaments which it was formerly customary to use in inflammatory conditions of all kinds were classified in the old therapeutics under the name of antiphlogistics, or remedies which combat inflammation (refrigerants, temperants). In this group were included the astringents, bleeding and other methods of local bloodletting, hydrotherapy (Priessnitz), cold, mercury and potassium nitrate. Inflammations due to infection are now treated with antiseptics (carbolic acid, iodoform, camphor, alcohol, salicylic acid, calomel). Astringents with antiseptic action are also employed (silver nitrate, copper sulphate, lead acetate, alum, tannin, cold). Moreover, inflammation is not a disease but, like fever, is to be regarded as a reactive natural healing process which in itself does not need to be combated.

Drugs which have an action opposed to the antiphlogistics were formerly classified as calefacients, i.e., heating agents. They are indicated in abnormal sinking of the body temperature (freezing, loss of blood, last stages of poisoning and infectious diseases). In addition to the external and internal employment of heat (warm bandages, warm drinks), the calefacients also include caffeine, which rapidly increases the temperature from 0.5 to $1^{\circ} \mathrm{C}$. Small doses of alcohol exert a similar effect.

Anticatarrhalics.-All of the drugs used in the treatment of catarrhs were formerly classified as anticatarrhalics. The group included particularly the astringents, expectorants, antiseptics and resorbents (alkalies).

Antidyscratics.-The terms antidyscratics, alterants and metasyncritics wereused in the older therapeutics todesignate a series of so-called blood-purifying medicines which were employed empirically in the treatment of different dyscrasias and cachexias. The group was further subdivided into antiscrofulous, anticarcinomatous, antisyphilitic, antiscorbutic, antarthritic, antirheumatic. Arsenic, mercury, iodine, phosphorus, sulphur, antimony, the alkalies and the so-called "wood-drinks" for man (sarsaparilla, sassafras) were regarded as alterative drugs. The action which is really obtained from these drugs is partly specifically antiseptic (mercury, iodine), partly resorbent (arsenic) and partly diuretic or diaphoretic (alkalies, hot water). 


\section{DRUGS THAT KIIL PARASITES. ANTIPARASITICS}

\section{EXTERNAL ANTIPARASITICS. ANTEPIZOA}

Synonyms: Antiscabious, antipsorics, antipediculous, antiphthiriacs; mange remedies.

Parasites of the Skin.-The numerous epizoa infesting the skin of the domesticated animals which are to be combated with antiparasitic remedies may be divided into two groups: animal and vegetable. The animal parasites include the mange mites, of which there are four species: sarcoptes, dermatocoptes, dermatophagus and dermatoryctes; the acarus mites [demodex folliculorum], fleas, lice, hair and feather insects [mallophagæ], ticks, forest flies, bird mites, harvest mites, œstrus larvæ, gad flies, flies and gnats; also the different bird mites: feather-follicle mites, feather-quill mites, feather mites, pigeon mites, etc. Of the vegetable skin parasites, the most important are trichophyton tonsurans (herpes) and achorion Schönleinii (favus).

Action of the Antiparasitics.-The antiparasitic medicines operate upon the skin parasites by different processes. Some apparently act like the specific nerve poisons, stunning and paralyzing the parasites. To this group belong the ethereal oils, nicotine, veratrin, carbolic acid, creolin and other aromatic substances of the benzol series, and hydrogen sulphide. Other antiparasitics act like the caustics, particularly the mercurial and arsenical preparations. If, for example, a louse is removed from the skin after the application of mercurial ointment and examined under the microscope, there is observed a corrosive inflammation of the digestive tract which is manifested by a bloody, profuse diarrhœa. The alkalies, soda lye, potash lye, caustic lime and soap, dissolve the external chitinous layer of the mange mites. Finally, some agents operate in a mechanical way by closing the air canals, as occurs, for instance, in the use of fatty oils on ticks.

Classification.-The external antiparasitics may be divided 160 
either according to their action into mange remedies, remedies against fleas, lice, ticks, gad flies, etc., or according to their chemical composition. The chemical subdivisions include antiparasitics from the group of ethereal oils: Peru balsam, styrax, turpentine oil, oil of anise, oil of caraway, flores pyrethri, petroselinum; alkaloidal antiparasitics: nicotine, veratrin, cevadilla seed, staphysagria; benzol derivatives: creolin, lysol, bacillol, carbolic acid, tar, creosote, salicylic acid,naphthalin, naphthol; metallic remedies: mercurial preparations, arsenic, sulphur; alkalies: soap, soda, potassium, caustic potash, caustic soda, caustic lime. The first mentioned form of classification is most suitable for practical therapeutics; according to this method the antiparasitics are divided into the following groups:

Mange Remedies.-The most important are creolin, tobacco, arsenic, corrosive sublimate, Peru balsam, carbolic acid, tar, creosote, lysol, bacillol, sulphur, soap, soda, potassium, lime. The following baths or "dips" are used in the treatment of sheep scab:

1. Fröhner's bath. Apply creolin liniment (creolin 1, alcohol 1, green soap 8) three to five days, then bathe twice in $2 \frac{1}{2}$ per cent. creolin solution.

2. Gerlach's bath. (a) Preparatory bath: potash 2, lime 1, water 50. (b) The mange bath proper: 3 per cent. tobacco decoction (Roloff's modification: 5 per cent. tobacco decoction).

3. Zuindel's bath: crude carbolic acid 1.5, lime 1, soda 3, soft soap 3, water, 260. A modification of this bath is to use 2 per cent. tobacco decoction in place of the water.

4. Tessier and Matthieu's bath: arsenic 1 to 1.5, iron sulphate or alum 10 to 15 , water 100 .

[Lime and sulphur dip: Mix 8 pounds of unslaked lime and 24 pounds of flowers of sulphur, add 30 gallons of water and boil for not less than two hours. Allow sediment to settle, draw off the clear liquid and dilute up to 100 gallons. Use at a temperature of 100 to $110^{\circ} \mathrm{F}$. and keep each sheep in the dip two minutes, ducking its head at least once; repeat in 10 days.

Tobacco and sulphur dip: Take one pound of tobacco leaves for each 6 gallons of dip desired, place in a covered boiler, cover 11 
with cold or lukewarm water and let stand 24 hours; bring to a boil the evening before using, and let stand over night. Take 1 pound of flowers of sulphur for each 6 gallons of dip desired and mix in a bucket with water to the consistency of gruel. When ready to use, strain the tobacco infusion, expressing the liquid from the tobacco leaves; mix the infusion with the sulphur gruel, and add sufficient water to make the desired quantity of dip. Use at a temperature of 100 to $110^{\circ} \mathrm{F}$. and keep each sheep in the dip two minutes, ducking its head at least once; repeat in 10 days. Extracts of tobacco and nicotine solutions are on the market which may be used in place of the tobacco leaves, thus simplifying the preparation of the dip. These substances should be used in a quantity which will produce a mixture containing not less than five one-hundredths of one per cent. of nicotine and 2 per cent. of flowers of sulphur.]

Remedies against Acarus Mites [Demodex].-Peru balsam, creolin, corrosive sublimate, sulphurated potash, cantharides ointment, oil of caraway, formaldehyde, bisulphide of carbon.

Remedies against Lice.-Mercurial ointment, creolin, lysol, bacillol, arsenic, tobacco, corrosive sublimate, insect powder, stavesacre seeds, cevadilla seeds, white hellebore, aniseed. These are also employed against the hair parasites [mallophagæ].

Remedies against Fleas.-Persian insect powder.

Remedies against Feather Parasites.-Oil of anise, insect powder, petroselinum, Peru balsam, creolin. The same remedies are used against the other skin parasites of birds.

Remedies against Ticks.--Olive oil, turpentine oil, carbolic acid in oil, creolin in oil.

[Arsenic has been found very effective for destroying ticks on cattle. The solution is prepared as follows: 25 gallons of water are placed in a caldron and brought to a boil; 24 pounds of sodium carbonate are added and dissolved by stirring; 8 pounds of arsenic are then added and the mixture stirred until it is dissolved. The fire is then drawn and when the solution has cooled down to $140^{\circ} \mathrm{F}$., one gallon of pine tar is slowly added and thoroughly mixed with the solution by stirring. Sufficient water is at once added to make 
the total volume of the solution 500 gallons. The regulations of the Bureau of Animal Industry require that when the solution is to be used as a dip for cattle which are to be shipped out of the quarantined area 25 pounds of sodium carbonate and 10 pounds of arsenic shall be used for each 500 gallons. In tick eradication, the solution is applied as a spray or in the form of a dip or bath, repeated every two or three weeks.]

Remedies against Forest Flies.-Creolin, tobacco, mercurial ointment.

Remedies against Estrus Flies.-Tar, creolin, asafœtida, petroleum.

Remedies against Herpes and Favus.-Salicylic acid, creolin, creosote, tincture of iodine, tar, mercurial ointment, ammoniated mercurial ointment, corrosive sublimate, carbolic acid.

Drugs.-1. *Creolin. Creolin (creolinum anglicum) is the most used antiparasitic. In the treatment of sheep scab it is applied for several days in the form of a 10 per cent. liniment: creolin and sapo mollis, 1 part of each, alcohol 8 parts. This is followed by a bath or dip in a $2 \frac{1}{2}$ per cent. solution of creolin, which is repeated in 8 days. Against sarcoptic mange of the horse and dog, a 10 per cent. liniment of creolin, soap and alcohol is also employed. A 10 to $\mathbf{1 5}$ per cent. alcoholic solution is used against acarus [demodectic] mange. Washings with 3 per cent. solution in water are employed against lice, forest flies, etc. Creolin ointment is used against herpes, chicken mange and ticks. Birds infested with vermin may be sprayed with a 1 per cent. solution of creolin in water or given a bath in a warm $1 / 2$ per cent. creolin solution, followed by a bath in water. ${ }^{*}$ Lysol $^{1}$ and ${ }^{*}$ bacillol ${ }^{1}$ have a similar action.

2. Pix liquida. Tar. A very good remedy against horse mange in the form of Vienna tar liniment: pix liquida and sulphur sublimatum, 1 part of each; sapo mollis and alcohol, 2 parts of each; also used against mange of dogs, herpes, etc., in ointment or in alcohol $(1: 10)$.

[' ${ }^{1}$ iquor cresolis compositus, which is official in the U. S., is essentially the same as these compounds.] 
3. Creosotum. Creosote. A good but very poisonous remedy against mange of the dog and horse in the form of creosoted oil $(1: 10)$.

4. Phenol. Carbolic acid. A remedy against sheep scab, usually in combination with tobacco.

5. *Tabacum. Tobacco. A remedy against sheep scab and mange in horses. The tobacco dip or bath for scab or mange is $\mathbf{a}$ decoction of $71 / 2$ kilograms [20 pounds] of tobacco in 250 liters [250 quarts] of water, to which is added 1 kilogram [2.68 pounds] each of pure carbolic acid and potash. A 1 to 5 per cent. decoction of tobacco or of tobacco-lye is also used against lice, fleas, etc.

6. Arseni trioxidum. Arsenic. The strongest but also the most poisonous remedy against scab in sheep and mange in horses. The arsenical dip or bath for sheep is ordinarily a 1 per cent. solution (combined with potash, alum, iron sulphate or aloes). As a wash for mange in the horse a 1 per cent. solution of arsenious acetate is used.

7. Hydrargyri chloridum corrosivum. Corrosive sublimate. A very powerful but also extremely poisonous antiparasitic against mange mites, acarus, herpes, lice, etc.

8. Unguentum hydrargyri. Mercurial ointment. A good remedy against lice. Formerly used also against sheep scab.

9. Balsamum Peruvianum. Peru balsam. A good and mild but expensive mange remedy for dogs; used undiluted or in alcohol (1:1 to 10$)$. A specific against acarus [demodectic] mange. The modern substitutes for Peru balsam: *perugen (artificial Peru balsam), *peruskabin and *peruol (synthetic benzyl benzoate) have a similar action.

10. Sulphur lotum, sulphur præcipitatum, sulphur sublimatum. Sulphur. A specific against mange of cats in the form of Helmerich's ointment ( 2 parts sulphur, 1 part potash, 10 parts lard).

11. *Potassa sulphurata. Liver of sulphur. A specific against acarus mange in the form of 1 to 2 per cent. baths.

12. *Insect powder. The most used remedy against fleas, feather insects, and fowl mites. 
13. Oleum anisi. Principal remedy against vermin of house birds; sprinkled or sprayed in 1 per cent. solution. Oleum cari has the same action.

14. Oleum terebinthinæ. Oil of turpentine. A specific against ticks.

15. Acidum salicylicum. Salicylic acid. A specific against herpes in 5-10 per cent. solution in alcohol.

16. Alkalies. These are used to assist the action of mange baths or dips.

\section{WORM REMEDIES. ANTHELMINTICS}

Synonyms: Vermifuges, antentozoa, antitænics; worm-expelling, wormdestroying remedies; tapeworm remedies.

Intestinal Worms.- Not all entozoa can be expelled from the body by medicines. Distomæ and echinococci in the liver and lungs, cysticerci and trichinæ in the muscles, giant palisade worms (Eustrongylus gigas) in the pelvis of the kidneys, strongylus armatus in aneurisms in the anterior mesenteric artery, cœnuri in the brain, and some intestinal worms are not accessible to medicaments. But most of the intestinal worms can be effectively removed by medicines. The parasites of the digestive apparatus of greatest importance are:

1. Tapeworms: tænia cucumerina, serrata, marginata, cœnurus and echinococcus, and bothriocephalus latus in dogs; tænia perfoliata, plicata and mamillana in horses; tænia expansa and ovilla in sheep; tænia expansa, denticulata and alba in cattle; tænia expansa in goats; tænia crassicollis and elliptica in cats; tænia infundibuliformis, etc., in birds.

2. Round worms: ascaris megalocephala in horses, ascaris marginata in dogs, ascaris mystax in cats, ascaris lumbricoides in cattle and swine, heteracis inflexa, etc., in birds.

3. Palisade worms: strongylus armatus and tetracanthus in the intestinal canal of the horse; strongylus contortus in the abomasum of sheep ${ }^{2}$ (stomach-worm disease); dochmius trigonocephalus in the intestine of the dog.

[2 Also affects calves in the southern part of the United States.] 
4. Seat-worms: oxyuris curvula, vivapara and mastigodes in horses; oxyuris vermicularis in the dog.

5. Spiroptera sanguinalenta in the stomach of the dog.

Another entozoön of the digestive apparatus is the gastrus larva, which is found in the stomach of the horse and which is resistant to most of the worm medicines. The entoparasites of the respiratory apparatus which are at least partially accessible to treatment include: cestrus ovis in the nasal cavities and nasal sinuses of sheep; strongylus filaria in sheep (lung-worm disease), strongylus micrurus in calves, strongylus paradoxus in swine; pentastomum tænioides in the nasal cavities of the dog; syngamus trachealis in fowl. In addition to these, vegetable parasites (moulds, actinomyces) also occur in the air passages.

Action of Worm Remedies.-The anthelmintics are generally regarded as specific nerve poisons which kill the entozoa immediately or which temporarily stupefy and paralyze them, in consequence of which they become detached from the intestinal mucous membrane and are in part expelled and in part digested. According to their chemical properties, anthelmintics may be classified as ethereal oils (turpentine oil, oleoresin of aspidium, oleum tanaceti); alkaloids (areca nut, pomegranate bark); glucositic acids (santonin, mallotoxin, kosin, filicic acid); benzol derivatives (creolin, tar, creosote, picric acid, naphthalin, benzine) and metals (arsenic, tartar emetic, copper oxide). For therapeutic purposes, it is most desirable to classify the anthelmintics according to the species of worms against which they are used, as follows:

Tapeworm Remedies: kamala, aspidium, oleoresin of aspidium, areca nut, kousso, pomegranate bark, copper oxide, potassium picrate.

Round-worm Remedies: tartar emetic, arsenic, santonin, santonica, turpentine oil.

Remedies against Lung Worms: turpentine oil, creolin, tar.

Remedies against Oxyuri: garlic, vinegar, soap, [quassia].

Remedies against Gastrus Larvæ [Bots]: bisulphide of carbon.

Against strongylus armatus and tetracanthus, also dochmius, spiroptera, and other intestinal worms in the horse, one of the 
aforementioned round-worm remedies is used; in dogs, one of the tapeworm remedies.

Drugs.-1. *Kamala. A tapeworm remedy for dogs. It is also a drastic cathartic and the simultaneous administration of a purgative is therefore not necessary. Dose for large dogs, 5-15, $3 i$ to iv; small dogs, 2-5, grs. $\operatorname{xxx}$ to $3 \mathrm{i}$; cats and chickens, $1-2$, grs. $\mathrm{xv}$ to $\mathrm{xxx}$; pigeons and parrots, $0.5-1$, grs. vij to $\mathrm{xv}$.

2. Oleoresina aspidii. Oleoresin of aspidium. A very effective but very poisonous tæniacide. Dose for large dogs, $2-5$, grs. $\mathrm{xxx}$ to $3 \mathrm{i}$; small dogs, $0.2-1$, grs. iij to $\mathrm{xv}$.

3. *Areca. Areca nut. Betel nut. A very good remedy against tapeworms and round worms. Dose for horses, 100-200, 勈 to vj; dogs, 5-10, 3 i to ijss; sheep, 5-10, 3 i to ijss; swine, 5-15, $3 \mathrm{i}$ to iv; chickens and geese, $2-4$, grs. $x \times x$ to $3 \mathrm{i}$; pigeons, $0.5-1$, grs. vij to xv.

4. Antimonii et potassii tartras. Tartar emetic. A valuable anthelmintic against tapeworms and round worms of horses. Dose, 10-15, 3 ijss to iv, per day in the drinking water for 2 to 4 days.

5. Oleum terebinthinæ. Oil of turpentine. A very good remedy against round worms in horses ( $50, \bar{\zeta}$ iss).

6. Arseni trioxidum. Arsenic. The same. Dose for horses, 1-3, grs. xv to $x{ }^{3}$ The new arsenical preparations, *Atoxyl and *Salvarsan, have proven effective remedies against trypanosomes and spirilla.

7. Santoninum. Santonin. A specific against round worms of horses and dogs. Dose for dogs, $0.05-0.2$, grs. $3 / 4$ to iij.

8. Carbonei bisulphidum. Bisulphide of carbon. A remedy against gastrus larvæ and round worms in horses. Dose, 8-12, $3 \mathrm{ij}$ to iij, in capsules, every hour until 4 doses have been given.

These eight remedies are sufficient for veterinary practice. The patient should be prepared for the anthelmintic by being starved a day before the medicine is administered. A few hours after the anthelmintic is given a purgative should be administered,

[ 3 Gerlach reported that 20 grains of arsenic caused acute diarrhcea in a horse.-Finlay Dun, Veterinary Medicines, 10th ed., p. 274.] 
except in the case of kamala and tartar emetic. To assist the action of the worm remedy, salted meat is given to carnivora, and sugar beets, carrots and raw potatoes to herbivora.

Other anthelmintics are: Cusso (dogs, 10-25, 3 ijss to vj), granatum (dogs, 5-50, 3 i to xij), pelletierinæ tannas (dogs, 0.2-0.4, grs. iij to vj), *tanacetum (horses and cattle, 50-100, 5 iss to iij), *cupri oxidum (horses, 2-15, grs. xxx to 3 iijss; dogs, 0.05-0.1, grs. $3 / 4$ to jss; sheep, $0.5-1$, grs. vij to $x v$ ), *acidum picricum and *potassii picras (lambs, of the former, 0.1-0.2, grs. jss to iij; of the latter, 0.5-1, grs. vij to xv), chloroformum (dogs, 1-4, npxv to $3 \mathrm{i}$ ), *creolin, naphthalenum, pix liquida, creosotum, benzinum, *petroleum, *absinthium, asafcetida, *allium and pepo. 


\section{DISINFECTANTS. ANTISEPTICS}

Synonyms: Asepties, colyseptics, antizymotics, antifermentatives, antiputrids, conservants, preservants; putrefaction and fermentation-combating remedies; conserving remedies, preserving remedies.

\section{DISINFECTION IN GENERAL}

Historical.-The practical application of disinfection, i.e., the destruction of pathogenic or putrefactive bacteria or the prevention of their growth, is very old. In this connection, it is only necessary to mention the unrivalled technique used by the ancient Egyptians in embalming the body. The scientific foundation of disinfection, however, is of more recent date. The new era began with the investigations of Pasteur concerning the yeasts and with the work of Lister, who in $\mathbf{1 8 6 7}$ for the first time studied the effect of disinfection upon the healing of wounds and introduced carbolic acid as a disinfectant. The further development of disinfection is closely connected with bacteriology and its more recent experimental discoveries, with which the names of Pasteur and $R$. Koch especially are associated. The first fundamental work of the latter was published in 1881 (Ueber Disinfektion, Mitteilungen aus dem Kaiserl. Gesundheitsamte, 1881). From this time on the knowledge of disinfection is so intimately related to bacteriology that to a certain degree it forms a part of that subject. And since the science of bacteriology is in a state of continual progressive development, it naturally follows that the present principles of disinfection may undergo certain changes in the future. To direct attention to the incomplete and unsettled condition of our present knowledge and conception of disinfection at the oustet is one of the most important tasks of general therapeutics. It consequently follows that a conclusive statement of our knowledge of disinfection cannot be made. This is the less feasible because the infectious agents of some of the important plagues (foot-and-mouth disease, contagious pneumonia of horses, influenza, pox, etc.) have not as yet been demonstrated bacteriologically, and the knowledge concerning disinfection for these diseases will, therefore, have to be 
subsequently entirely revised on a scientific basis. Nevertheless, the investigations which have been made up to this time have furnished a series of important viewpoints concerning general and special disinfection which may be regarded as a permanent acquisition and as a preliminary foundation for a rational and scientific system of disinfection. The foundation of the modern antisepsis and disinfection must be explained first of all. This explanation will include the meaning of the different terms, antisepsis and asepsis, colyseptics (inhibit development) and antiseptics (destroy bacteria); the methods of bacteriological investigation; the differences in the individual bacteria and disinfectants; the differences between bacilli and spores; method of action, form, concentration, application and properties of the individual medicines; the relation of the animal body or the infected object to the disinfectant; the coöperation of certain factors (temperature, light); the importance of cleaning and preparation, and the time and continuance of the disinfectant action.

Antisepsis and Asepsis.-Starting with the conception that infection of wounds could be prevented by the employment of antiseptics-i.e., drugs which destroy bacteria-and by a carefully applied dressing, Lister in 1867 recommended carbolic acid in connection with the dressing named after him as a method of disinfecting wounds. Lister's dressing was applied as follows: After the wound was cleaned with a 2 to 5 per cent. solution of carbolic acid in water, and the air aboveit disinfected with a spray of the carbolic acid solution, it was covered with a piece of carbolized silk or cotton (protective); over this was laid a thick layer of carbolized gauze or cotton, which was covered with a piece of imperviouscotton material (mackintosh), and over all was placed a bandage of moist carbolized gauze. In Germany, the Lister dressing was introduced into general use between 1872 and 1875 . Later, the carbolic spray was omitted and the dressing simplified. In 1880, iodoform was substituted for carbolic acid and the dry bandage took the place of the moist. Subsequently, iodoform was replaced in part by corrosive sublimate, creolin, lysol, tannoform, tincture of iodine, and other disinfectants. 
In recent times the use of antiseptics has been limited in human surgery and for a time they were even given up entirely. Schimmelbusch and others contended that the development of germs in a wound could not be prevented with certainty by disinfectants even when they were applied as early as one minute after the infection; disinfection was therefore not only ineffective, but also harmful, because it irritated the tissues and increased the wound secretions. In place of the antiseptic wound treatment, the aseptic method was proposed. Antiseptic fluids are not employed in the latter method, but the wound is kept as dry as possible by means of sterilized tampons, or is irrigated only with sterile water or sterile physiological salt solution, and then covered with sterilized dressing material. The latter is sterilized in a special apparatus by prolonged (20 to 30 minutes) exposure to live steam of at least $100^{\circ} \mathrm{C}$. In a similar manner, the instruments are made germ-free. They are most certainly sterilized, however, by prolonged boiling in a 1 to 2 per cent. soda solution. Especial care is given to the disinfection of the operator's hands. After the nails are thoroughly cleaned, the hands are carefully brushed with soap and warm water, then washed with warm sublimate, carbolic acid or creolin solution and finally rinsed with 50 per cent. alcohol or soap spirit. Care is also taken to keep aseptic the entire operating room, the operation table, the clothing of the patient, operators, assistants and attendants, all of the utensils used and the area surrounding the wound (field of operation). Mull masks for the mouth and nose of the operator are even used.

The aseptic treatment of wounds was regarded as an important advance in human surgery because it left the healing of the wound to the natural protective forces of the tissues (leucocytes, bloodserum) and only guarded against external disturbances. More recently, after Henle and others had shown that, contrary to Schimmelbusch, local disinfection of wounds within the first hours is very effective, and after it had been proven by statistics that the results of the aseptic method were in no way more favorable than the antiseptic, numerous surgeons changed from the purely aseptic method to antisepsis (disinfection of the skin with tincture of 
iodine). Moreover, disinfection of the hands was difficult and uncertain and the introduction of sterilized operating gloves did not improve conditions.

The aseptic method is not very well suited to veterinary surgery. To this I have already directed attention in another place (General Surgery). In veterinary practice, old infected wounds are usually met with and for these thorough antisepsis is necessary rather than asepsis. For fresh operation wounds in the domestic animals the aseptic method is only exceptionally applicable, as for example in a clinic; and even in well-equipped veterinary hospitals the greatest difficulties are encountered in maintaining asepsis, especially in horses, as a dressing can be applied only in a very limited number of cases and infection of the wound during the operation frequently cannot be avoided even with the greatest care. Moreover, in addition to the contact infection, the air infection (dust, hair) is of great import, and against this asepsis has proven powerless. For these reasons, antisepsis is to be preferred in veterinary practice to asepsis as the more certain method.

Colyseptics and Antiseptics.-A pathogenic or putrefactive microörganism can be made harmless in two ways. Its vital activity can be destroyed or its development can be prevented. In the first instance the action is antiseptic; in the latter, colyseptic. Drugs with antiseptic properties are naturally more valuable than those which have only a colyseptic action. Therefore, a strong antiseptic action is the first essential in a good disinfectant; it must not only prevent the development and multiplication of pathogenic bacteria but must destroy their vitality. As a general rule, the antiseptics exert only a colyseptic action when sufficiently diluted. On the other hand, there are several disinfectants which are only colyseptic in the strongest concentration and have no antiseptic action or only a very weak one.

The division of the disinfectants into antiseptics and colyseptics is only possible upon the basis of bacteriological experiments. In order to test the colyseptic properties of a substance, bacteria capable of development are placed upon a suitable culture: 
media which has been previously impregnated with the drug under examination. If the bacteria fail to develop characteristic colonies upon the media (gelatin, bouillon, etc.), then it is to be assumed that the presence of the drug has prevented the growth of the organisms. However, it cannot be concluded from the experiment that the vitality of the organisms is destroyed. This fact is only established when a pathogenic organism which has been thus treated is inoculated into a suitable experimental animal and no infection is produced. Only in the latter case can antiseptic properties be attributed to the drug. Non-pathogenic bacteria are tested by permitting them to dry upon silk threads and placing the threads in a solution of the substance under test. After a time the threads are removed from the solution, washed in water, and placed upon suitable culture media. If the substance being tested actually possesses antiseptic properties no growth will occur.

Relative Rank of the Disinfectants.-In the beginning, the individual disinfectants were tested bacteriologically in an endeavor to discover a drug which would destroy all known bacteria when greatly diluted, but it was found that there is no such universal disinfectant. On the contrary, it was observed that specific antiseptics are required to destroy individual species of bacteria, just as certain antipyretics are required for the different types of fever. For instance, corrosive sublimate is the most powerful disinfectant for the virus of anthrax, but has only a very weak action on tubercle bacilli and is much less effective than other drugs (creolin, lysol, alcohol, formaldehyde) for superficial disinfection of the skin. Carbolic acid is relatively ineffective against the tetanus bacillus, rabies virus, anthrax spores and tubercle bacilli. Therefore, in disinfection the different drugs and bacteria must to a certain extent be considered individually. In a general way, it can only be said that in the case of those microorganisms which exist in two different forms, namely, the continuous (spore) and vegetative (bacillus), the spores require much more powerful antiseptics than the bacilli. Here, again, the individual species of spores and bacilli show considerable differences in their ability to resist the action of the same drug. It is, 
therefore, difficult to make up a comparative table of the individual drugs. It can only be stated in a very general way that the strongest disinfectants, which also destroy spores, are corrosive sublimate and the silver salts (silver nitrate); chlorine, bromine and iodine; creolin, lysol and some of the other newer cresol preparations; formaldehyde and wood tar. Contrasting with these are the weaker antiseptics, which kill only the spore-free bacteria: coal tar, carbolic acid, salicylic acid, aniline dyes, boric acid, calcium, lyes and acids. Iron sulphate and sulphuric acid, formerly highly valued as antiseptics, possess almost no antiseptic properties. The aforementioned disinfectants are arranged in the following table according to the dilutions in which they are efficient, the dilutions given being the average of the different bacteriological observations;

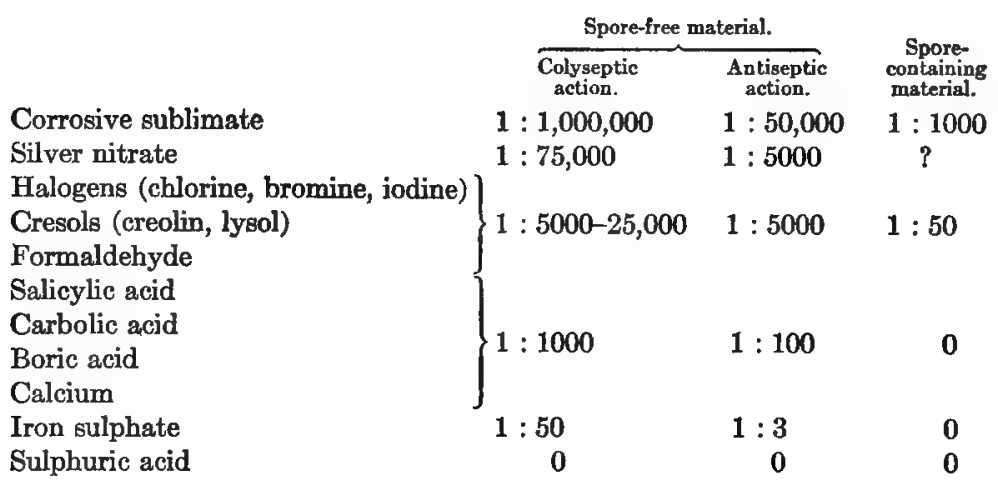

Resistance of the Individual Infectious Agents.-This is very variable. Many infectious agents are very readily destroyed by disinfectants. As a consequence, for many of the infectious diseases the employment of the weaker disinfectants (lime, soap, tar) is sufficient. This is due in part to the fact that many of the pathogenic organisms do not form spores. The infectious agents which are easily destroyed are the anthrax bacilli, the bacilli of swine erysipelas, the bacteria of hemorrhagic septicæmia of cattle, the bacteria of fowl cholera, and the virus of foot-and-mouth disease, vesicular exanthema, pox and rinderpest. On the other hand, 
anthrax spores, tubercle bacilli, tetanus spores, black leg spores, and the contagion of lung plague and rabies are destroyed with difficulty. For the purposes of practical disinfection, the more important infectious diseases can therefore be classified in two groups, the one including those for which a mild disinfectant is sufficient and the other those which require a strong disinfectant. The following groups are arranged on this basis:

\section{A. Requiring strong disinfectants:}

Anthrax spores.

Tetanus spores.

Tubercle bacilli.

Black leg spores

Lung plague virus.

Rabies virus.

B. Requiring mild disinfectants:

Anthrax bacilli.

Swine erysipelas bacilli.

Glanders bacilli.

Bacteria of hemorrhagic septicæmia.

Foot-and-mouth disease virus.

Pox and rinderpest virus.

Staphylococci and streptococci occupy a position between these two classes. They are much easier to destroy than spores and spore-containing bacilli, but are considerably more resistant to disinfectants than the spore-free organisms. For these reasons, it is necessary to use the more powerful disinfectants (corrosive sublimate, creolin, tincture of iodine) in the antiseptic treatment of wounds in order to destroy the pus cocci (staphylococcus pyogenes, streptococcus pyogenes).

Method of Action of the Disinfectants.-It has already been stated that infectious material may be rendered ineffective either through destruction of its vitality or through inhibition of its development. In either case the method of action may be very different. In general, disinfection is the result of the following processes:

1. By coagulating the bacterial albumins (mycoproteins) the 
metallic salts, particularly corrosive sublimate and silver nitrate, act as antiseptics (formation of a precipitate of metallic albuminate). Formaldehyde acts in the same manner.

2. A specific toxic action, narcotic to a certain extent, is attributed to the benzol derivatives, especially the cresols (creolin, lysol, tar, creosote) and carbolic acid.

3. By oxidation, potassium permanganate $\left(\mathrm{KMnO}_{4}\right)$, potassium chlorate $\left(\mathrm{KClO}_{3}\right)$, hydrogen peroxide $\left(\mathrm{H}_{2} \mathrm{O}_{2}\right)$, and other drugs act as disinfectants.

4. Reduction (withdrawal of hydrogen). Chlorine and many chlorine compounds operate by reduction. Corrosive sublimate, zinc chloride and iron chloride, for example, act in part in this way. The chlorine withdraws hydrogen from the bacteria, thus breaking up and decomposing the albumin molecules, while the chlorine and hydrogen combine to form hydrochloric acid; the chlorine also decomposes the water, setting free nascent oxygen, which oxidizes the bacteria.

5. Through a change of the reaction, most antiseptics act as disinfectants, especially the acids and acid chlorides (also the acid-reacting corrosive sublimate). Bacteria grow upon a weakly alkaline or neutral culture media and merely a change of reaction is sufficient to retard their development. Growth is also effected by a considerable increase of the alkalinity (alkalies).

6. By dissolving the cell membrane, alkalies, organic acids (vinegar) and soaps operate as disinfectants.

7. Precipitation. Corrosive sublimate and the other metallic salts (iron sulphate) precipitate the bacteria from the fluids in which they are suspended.

8. Absorption. Freshly heated charcoal absorbs gases and acts as an antiseptic and deodorizer.

9. Withdrawal of water. Concentrated salt solutions (e.g., sodium chloride) and glycerin act in this way. Finally, a purely mechanical fixation is produced by certain substances like oil paints, tar and pastes.

As will be seen from the foregoing, the method of action of a disinfectant may often be multiple. Corrosive sublimate, for 
example, operates by coagulating albumin, by its acid reaction, by precipitation and by the action of its chlorine.

Disinfectants and the Infected Objects.-The results obtained by experiments in bacteriological laboratories cannot be applied to disinfection in practice without further consideration (compare iodoform!). Between the destruction of bacteria in artificial cultures and the disinfection of a living body, a wound, a stable or a manure pit there frequently exist very considerable differences. Therefore, in practice it is well to consider the inter-action between the disinfectant and the object to be disinfected; both, the disinfectant as well as the object, may under certain circumstances suffer changes in consequence of opposing influences which will entirely defeat the purposes of the disinfection.

For the living body, the first point to be determined is whether the disinfectant is poisonous or non-poisonous. Strictly speaking, there is no disinfectant which is strongly antiseptic and at the same time absolutely non-toxic for the animal body. It is rather the rule that the toxicity of a disinfectant increases with the strength of its antiseptic power, as is observed especially in connection with corrosive sublimate. But if the term poisonous is used in the ordinary sense, and is to be understood to mean harmful results from small quantities of the disinfectant, or from weak dilutions used in the usual way for practical disinfection, then it can be said that there are disinfectants which are not poisonous. As examples, boric acid, salicylic acid, aluminum acetate and creolin may be mentioned. In regard to the former denials of the non-toxicity of the latter drug, the investigations of von Behring and experiences in practice agree that creolin may be employed as a disinfectant without any danger of poisoning. As poisonous antiseptics may be mentioned carbolic acid, creosote, the free cresols, corrosive sublimate, formaldehyde, chlorine, bromine and iodine. Corrosive sublimate is the most poisonous; it is forty times as poisonous as carbolic acid. According to von Behring, the toxicity of a disinfectant for the animal body may be calculated from the action of the drug upon anthrax bacilli suspended in blood-serum; most disinfectants are five to seven times 
more poisonous for the animal organism than for anthrax bacilli. Poisoning from antiseptics results from their internal, subcutaneous and epidermatic use, especially from the use of corrosive sublimate to disinfect the uterus of the cow and carbolic acid to disinfect wounds of cats. Disinfection with chlorine and bromine is very dangerous for man and animals.

The blood exerts a very strong modifying influence upon the disinfectant action of many antiseptics. Corrosive sublimate, for example, destroys anthrax bacilli in blood only in a concentration of $1: 2000$ and after prolonged action, while it destroys the same organism in water immediately and in a dilution of $1: 50,000$. The cause of this surprising difference is to be found in the fact that the albumin in solution in the blood is precipitated by the sublimate in the form of albuminate of mercury, which retards the penetration of the sublimate into the deeper layers of the blood. Similar observations have been made in connection with silver nitrate, carbolic acid, creolin and other drugs. While creolin is effective against anthrax bacilli in bouillon in a dilution of $1: 10$,000 , a concentration of $1: 200$ is required to destroy anthrax bacilli in blood-serum. Since in the disinfection of wounds one has to do in part with blood disinfection, these relations should be given consideration in the antiseptic treatment of wounds and blood should be as completely removed from the wound as possible.

In using disinfectants upon the skin or upon wounds, it must be remembered that many of them are caustic in strong concentration (sublimate, silver nitrate, carbolic acid) and are always irritant, even in weak solution (sublimate, formaldehyde). This consideration led to the substitution of asepsis for antisepsis in human medicine (see p. 170). Moreover, only a superficial disinfection can be obtained, since the disinfectants as a rule do not operate deeply (alcohol is an exception). A superior disinfectant for superficial disinfection of the animal body and one which is to be preferred to corrosive sublimate as well as carbolic acid is, according to von Behring, the English creolin. This is also true of formaldehyde and dilute alcohol (disinfection of the hands). 
Iodoform undergoes a peculiar decomposition when it comes in contact with pus;iodine is set free, and this free iodine is the cause of the antiseptic action of iodoform.

In the disinfection of stables, many forms of decomposition occur. This is especially the case with corrosive sublimate when it comes in contact with organic or albuminous substances, excrement, urine, and water. The disinfection of dried infectious material is particularly difficult; a detailed discussion of this point will be found in a succeeding section. The effective disinfection of infected dung and liquid manure is very difficult because the organic constituents and gases $\left(\mathrm{H}_{2} \mathrm{~S}, \mathrm{NH}_{3}\right)$ decompose some antiseptics; e.g., corrosive sublimate. Other disinfectants quickly lose their effect because they form ineffective combinations (change of caustic lime to acid phosphates, silver nitrate to silver chloride, salicylic acid to sodium salicylate).

Continuance of the Action of Disinfectants.-The rapidity of its disinfectant action is of essential importance in considering the value of any antiseptic. The shorter the action, the stronger usually must be the concentration. It is desirable in practice to continue the action of a quickly acting disinfectant as long as possible upon the object to be disinfected. Antiseptic irrigations of old wounds especially must be continued for a long time (for $1 / 4$ hour and over). Disinfection of stables with autan must continue seven hours. In disinfecting with chlorine and bromine a 24-hour action is necessary.

In general, a good disinfectant should destroy the bacteria acted upon within a few minutes. This standard cannot be complied with absolutely, particularly not in connection with the spore-containing bacteria, which always require a much longer time for destruction than the spore-free organisms. While carbolic acid, creolin, formaldehyde and corrosive sublimate in suitable concentrations destroy anthrax bacilli at once or at most within a few minutes, they show the following relations to anthrax spores:

Formaldehyde ( 2 per cent.) kills anthrax spores after 7 hours. Corrosive sublimate (17 per cent.) kills anthrax spores after 1 day. 
Creolin ( 3 per cent.) kills anthrax spores after 2 days. Carbolic acid (5 per cent.), anthrax spores were not killed after 20 days.

Influence of Temperature.-The disinfectant power of a drug can be considerably increased by the simultaneous operation of high degrees of temperature. For this reason, it is recommended that disinfectant fluids be employed as warm as possible. This applies particularly to the disinfection of the hands (warm water and soap and sublimate solution). Anthrax bacilli are destroyed at $3^{\circ} \mathrm{C}$. by a $1: 25,000$ solution of sublimate, while at $36^{\circ} \mathrm{C}$. they are destroyed by a four times weaker dilution, $1: 100,000$. Anthrax spores, which are not destroyed with certainty after 20 days by a 5 per cent. solution of carbolic acid at ordinary temperatures, are killed after 3 hours' exposure to the carbolic acid solution at $37^{\circ} \mathrm{C}$. Live steam kills them within the same period, and formalin vapor (1-2 per cent.) at $70^{\circ} \mathrm{C}$. after 5 minutes. Soda is entirely ineffective against these organisms at the ordinary temperature, but a $1 \frac{1}{2}$ per cent. solution at higher temperatures will destroy them as follows:

At $80-83^{\circ}$ C. after 10 minutes.

At $77^{\circ} \mathrm{C}$. after 15 minutes.

At $75^{\circ}$ C. after 20 minutes.

At $70^{\circ}$ C. after 30-60 minutes (von Behring).

Influence of Concentration.-The stronger the concentration the more effective in general is the disinfectant action of the antiseptic. An exception to this rule is creolin, which is most efficient in concentrations which favor the formation of a complete emulsion, especially $2-3: 100 ; 10$ to 20 per cent. solutions are not relatively as efficient because the greater part of the creolin is not emulsified and is therefore not entirely effective. The influence of concentration upon the action of an antiseptic is shown by the following figures:

Corrosive sublimate $1: 100$ kills anthrax spores after 80 minutes.

Corrosive sublimate 1 : 200 kills anthrax spores after 2 hours. Corrosive sublimate $1: 400$ kills anthrax spores after 4 hours. 
Corrosive sublimate 1 : 1000 kills anthrax spores after 24 hours (von Behring).

Influence of the Form of the Disinfectant.-The different conditions of aggregation exert a definite influence upon the degree of antiseptic action. Solid, insoluble bodies possess no disinfectant action because they cannot penetrate the mass to be disinfected. Disinfectants in solution in water are most effective. Alcoholic solutions are also very effective when the solvent is alcohol diluted with water. On the other hand, solutions in oil are not effective, as was demonstrated by Koch in connection with carbolized oil. Disinfectants in the gaseous form are very unreliable, except formaldehyde. Koch was the first to demonstrate the inefficiency of sulphurous acid, which was formerly so highly valued as a disinfectant. The practical value of chlorine and bromine in the gaseous form has been placed in great doubt by von Behring. Even calcium chloride is disinfectant in slight degree in the gaseous form only when the object to be disinfected is moist, when the bacteria are entirely superficial and when $11 / 2$ per cent. by volume of free chlorine is developed in the area to be disinfected. Many other superior antiseptics are not suitable for practical use because they are not soluble in water. These include, first of all, the ethereal oils, which in alcoholic solutions possess a very high disinfectant action. The behavior of iodoform, which is in powder form, is peculiar; it becomes antiseptic only after it is decomposed and iodine is set free.

Time of Disinfection.-In many cases it is very important that the disinfection be undertaken within a certain time. This applies especially to the disinfection of wounds. Fresh wounds are much easier disinfected than old, suppurating wounds. The prompt use of a suitable disinfectant (tannoform) on fresh wounds will prevent suppuration. On the other hand, the disinfection of old wounds is difficult and frequently impossible on account of the bacteria having penetrated deeply into the tissues. In anthrax, tetanus and rabies, disinfection should be undertaken at the earliest possible moment after the diagnosis is made, especially ' in anthrax, in order to prevent the easily destroyed bacilli from developing the highly resistant spores. 
Preliminary Preparation.-A thorough cleaning is an indispensable preliminary preparation for disinfection. This will make the infectious material more accessible to the antiseptic, so that a relatively simple and mild disinfectant will be sufficient, or it may entirely remove the infection. Water and soap alone are often sufficient for disinfection. The aseptic treatment of wounds was based entirely upon a careful cleansing of the wound, the instruments and the hands, and the exclusion of infection. Infected rooms, walls and other objects may be disinfected by thorough washing with warm water and soap. A preliminary disinfection must, however, precede the cleaning when diseaseproducing organisms may be scattered by the cleaning, or when the dirt cannot be collected in such a manner as to exclude the possibility of spreading the infection; in addition, when the cleaning is done without a preliminary disinfection those doing the work are in danger of infection. In these exceptional cases the work should be done in the following order: first, preliminary disinfection; second, cleaning; third, thorough final disinfection.

Cost of Disinfection.-In every disinfection the question of cost is an important consideration. In the interest of agriculture, the veterinarian is obliged to adopt the cheapest possible method of disinfection. For instance, the free use of iodoform, ichthargon, glutol or protargol by the veterinarian in the treatment of wounds must be regarded as a luxury. The use of silver nitrate as a disinfectant in outbreaks of disease is forbidden by its high cost. Carbolic acid must also be regarded as a relatively expensive disinfectant. A 5 per cent. solution of carbolic acid is ten times as costly as the much more powerful 1 per cent. corrosive sublimate solution and 3 per cent. creolin solution. Properly carried out, chlorine and bromine disinfection is very expensive. The cheapest disinfectants, and therefore the easiest to obtain, are lime, soda, potassium, soap and tar. Other cheap disinfectants are corrosive sublimate, creolin, lysol, solveol, solutol, bacillol and cresol-sulphuric acid. In the following table is given the present average wholesale price per pound of the most frequently used antiseptics or disinfectants: 
Burnt lime.................. per lb. $\$ 0.08$

Creolin.......................... per lb. $\quad .65$

Lysol. ...................... per lb. $\quad .67$

Carbolic acid................... per lb. $\quad .15$

Corrosive sublimate............... per lb. $\quad .69$

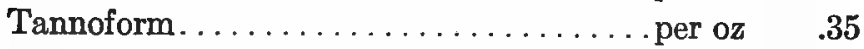

Iodoform.....................

Protargol.................... per oz. $\quad 1.25$

Aristol. .................... $\quad 1.80$

Ichthargon.................... per oz. $\quad 3.00$

Properties of Disinfectants. - The results of disinfection will depend upon other factors of importance. These include the quality and purity of the disinfectants. When calcium chloride is stored its chlorine content decreases and it finally becomes ineffective. Burnt lime, when exposed to the air, is gradually changed to the ineffective calcium carbonate. Calcium chloride and burnt lime must therefore be used in as fresh condition as possible. In regard to creolin, Henle and von Behring have demonstrated that the German creolin, in contrast with the English creolin, possesses no disinfection action worth mentioning. Finally, an effort should be made to carry out every disinfection with as little inconvenience as possible, avoiding all unnecessary disturbance of agricultural operations.

\section{DISINFECTION FOR INFECTIOUS ANIMAL DISEASES}

General.-Disinfection for the different infectious diseases of animals is regulated by the German veterinary sanitary law of June 26, 1909 (Supplement A to the instructions of the federal council of December 25, 1911), the law of February 25, 1876, concerning the spread of infectious materials by the transportation of cattle over railroads, and the regulations of the federal council based on this law and issued June 20, 1886, with the modification of July 26, 1899 (the disinfection with 5 per cent. carbolic acid solution is not only required, as formerly, for anthrax, rinderpest and foot-and-mouth disease, but also for glanders, swine plague and hog cholera); also by the more recent regulations with reference to fowl cholera (February 2 and July 4,1899) and the announcement of the federal council of July 16, 1904 (substitution of a 3 per cent. solution of the cresol-sulphuric acid mixture for the 
5 per cent. carbolic acid solution), by the rinderpest law (April 7, 1869), and by the military-veterinary instructions for the service horses of the army. These laws and regulations contain definite instructions concerning cleaning and disinfectant agents (water, soap water, soda lye, freshly-slaked lime, calcium chloride, solutions of carbolic acid and cresol, cresol-sulphuric acid mixture, corrosive sublimate, formaldehyde, fire), methods of cleaning and disinfecting, and the procedure to be followed in the different infections (anthrax, rabies, glanders, foot-and-mouth disease, lung plague, sheep-pox, swine erysipelas, swine plague, hog cholera, tuberculosis, mange, fowl cholera, fowl pest and rinderpest).

\section{SUPPLEMENT A TO THE INSTRUCTIONS OF THE FEDERAL COUNCIL, DECEMBER 25, 1911 (DISINFECTION IN INFECTIOUS DISEASES)}

\section{Regulations for Disinfection in Infectious Diseases}

I. General

\$1. Cleaning and disinfection is to be done according to these regulations under the observation and direction of the official veterinarian and under police supervision.

§2. The disinfection process includes cleaning and disinfection. The cleaning must regularly precede the disinfection; this does not forbid a preliminary disinfection before beginning the cleaning (see $\S 5$, No. $10 ; \S 6$, subdivision 2).

\section{Cheaning}

§3. Persons must wash with warm water and soap the hands and other parts of the body which may have been soiled; in case of necessity these parts must also be subjected to a preliminary disinfection ( $\$ 5$, No. 10$)$. The clothing and foot-gear, when not changed, are to be brushed with soap and water to remove attached dirt.

§4. Animals are to have the surface of the body, especially the hoofs and claws, cleaned of attached dirt by careful washing or some other suitable process. When necessary the hoofs and claws are to be trimmed out.

\$5. Stables and other places of accommodation are to be treated as follows:

1. Manure and other gross dirt, straw, feed residue, straw packing, and similar substances are to be removed and disposed of according to Nos. 9 and 10. Only the upper layer need be removed from the manure piles in 
sheep and cattle stables when in the judgment of the official veterinarian this is sufficient and when the veterinary sanitary regulations will permit.

2. Wood utensils, racks and mangers and board linings are to be taken out as far as is necessary. Wood-work with a torn or splintered surface must be removed to a sufficient extent to obtain a smooth surface. The pieces of wood removed and all decaying or rotten wood, or wood that is otherwise unsuitable for use, are to be burned.

3. From clay walls a layer of sufficient depth is to be removed. Damaged or loose parts of the plaster or rough-cast on the walls are to be removed and disposed of in the same manner as the manure.

4. Loose paving and planks in the floor are to be removed. The earth under the flooring which is wet with excretions must be dug out, and the material removed is to be disposed of in the same manner as the manure. Stone and sound wood-work in which the moisture has not penetrated deeply can be again used after the damaged parts have been removed.

5. In tightly joined (impervious) floors, in case of necessity, the damaged places in the binding material or in the material itself, or cracks in the latter, can be scraped out and the spaces, after thorough cleaning and disinfection, can be filled in with new material. Similar conditions in the walls, pillars, stall partitions, pits, troughs, gutters and canals may be treated in the same manner.

6. Lime or earth floors (puddled clay and the like) should have the upper layer removed and the moist places dug out. The part removed is to be treated like the manure.

7. Earth and sand floors, in so far as they are wet with excretions, are to be removed to the depth of at least 4 inches.

8. The ceiling, walls, fittings (mangers, troughs, racks, posts, pillars, stalls, doors, door posts, windows, etc.), floor, manure gutters, canals, troughs and pits are to be thoroughly cleaned by scouring with hot soda solution (a solution of at least 8 pounds of washing soda to 100 quarts of hot water) or hot soap solution (8 pounds of soft soap dissolved in 100 quarts of hot water). The cleaning is only to be regarded as complete when all excretions from diseased or suspected animals and all dirt have been removed and everything has an entirely clean appearance. When necessary, scouring sand may be used with the hot soda or soap solution. The cleaning must include all parts of the stable and other places where animals are kept. Special attention is to be given to depressions in the floor, corners, niches, joints, cracks, scratches, etc. In stables and other compartments, the ceilings are cleaned first, as a rule, then the walls and interior fittings and lastly the floors, manure gutters, etc.

When the official veterinarian considers it unnecessary, stable ceilings and the upper parts of the walls which have not been soiled with the eliminations 
from diseased animals need not be scoured with the soda or soap solution, but may be cleaned by thoroughly spraying with hot soda or soap solution or with hot water. Where hot soda or soap solution or hot water cannot be obtained in sufficient amount, water under strong pressure from a water supply pipe or from a fire hose, garden hose or some similar arrangement may be used, if in the judgment of the official veterinarian it will answer the purpose.

9. The manure and other dirt removed in cleaning, the straw, feed residue and other substances (see Nos. 1 to 7), blood, stomach and intestinal contents and other offal from slaughtered, dead and diseased or suspected animals are to be collected together upon the infected premises. In cases where the collection of the manure upon the infected premises is impossible or undesirable it may, with official veterinary approval and with proper care, be collected in a suitable place outside of the infected premises.

The soiled water from cleaning is to be collected in the manure pit or in some other general container on the infected premises.

10. When the manure and other dirt, the straw, feed residue, etc., removed in cleaning, and the dirty fluids from cleaning, cannot be collected on the infected premises or in another suitable place without danger of spreading the infection, then, if it is necessary to render those materials harmless, a preliminary disinfection must precede the cleaning, a suitable disinfection fluid being applied ( $\$ 11$ and $\S 15$ to 27 ). In these cases, care should be taken that the manure and other dirt, straw, feed residue, dirty water, etc., are not placed even temporarily, before the final disinfection, in locations where the contaminated water may drain into other premises, where the materials are accessible to strange persons or animals, or where the fluids can flow into springs, water courses or ather sources of water supply.

A disinfection is also to precede cleaning when there is danger that the persons doing the cleaning may be infected without previous disinfection, as in anthrax and glanders $(\$ \S 15,18)$.

§6. (Subdivision 1.) Utensils, clothing, and other objects are to be treated in the following manner:

1. Combustible articles of little value are to be burned.

2. Wood stable and driving equipment (feed boxes, buckets, brooms, forks, shovels, etc., feed carts, wagons, sledges, harness parts, wood shoes, etc.) are to be thoroughly scoured with hot soda or soap solution.

3. Utensils of iron and other metals (chains, rings, forks, shovels, currycombs, bits, muzzles, troughs, other feeding and watering vessels and other vessels, cages, etc.) are, in so far as they cannot be disinfected by fire (part III), to be thoroughly cleaned and then washed with hot water.

4. Leather and rubber articles (halters, girths, bridles, draught harness, saddles, straps, cushion covers, leather shoes, dog collars, whips, etc.) are to be scrubbed with soap and water. 
5. Articles of cloth or vegetable fibres (blankets, girths, halters, ropes, cushion covers, pieces of clothing, bed covers, etc.) are to be freed from dirt by scrubbing with soap and water.

6. Hair, wool, feathers, cushion packing, and similar substances are to be spread out in a thin layer and exposed to the air for three days, being turned as often as possible.

(Subdivision 2.) Under the conditions described in $\S 5$, No. 10, a preliminary disinfection of utensils, clothing and other objects is necessary.

$\$ 7$. The cleaning of railroad stock pens and similar places, staughter houses, ships and ferries is to be carried out as directed in $\$ \S 5$ and 6 .

§ 8. Stock-yards are to be cleaned by first collecting the manure dropped by the animals; then paved places are to be thoroughly cleaned with brooms and washed with water, and unpaved places are to be raked and harrowed level. Where necessary, the apparatus for tying-up is to be washed with water. yards.

\$9. Roads (alleys) are to be cleaned in the same manner as the stock-

$\S 10$. Places in pastures where animals stand (exercise places, milking stations, etc.) are to be cleaned in the same manner as the stock yards.

\section{Disinfection}

\section{Disinfectants}

811. (Subdivision 1.) The following are used as disinfectants:

1. Freshly slaked lime. This is obtained as follows: freshly burned lime is placed, without breaking up or powdering, in a capacious vessel and covered with about half the quantity of water; under these circumstances it breaks up into a powder with the generation of considerable heat and gas.

2. Milk of lime. This is used in concentrated and dilute form.

Concentrated milk of lime is prepared by adding slowly, with continual stirring, three liters [3 quarts] of water to one liter [1 quart] of freshly slaked lime.

Dilute milk of lime is prepared by adding slowly, with continual stirring, 20 liters [20 quarts] of water to one liter [1 quart] of freshly slaked lime.

If freshly slaked lime cannot be obtained, then the milk of lime is prepared by mixing with each liter of slaked lime, as it is found in a lime pit, 3 or 20 liters of water. In such cases, however, care must be taken to previously remove the upper layer of lime, which is changed by the influence of the atmosphere.

The milk of lime is to be shaken and stirred before it is used.

3. Calcium chloride solution. The calcium chloride should possess a pronounced odor of chlorine and should be stored in tightly stoppered bottles and protected from light. The solution is prepared by adding to each liter 
[quart] of calcium chloride, slowly and with continual stirring, 3 or 20 liters [quarts] of water (concentrated and dilute calcium chloride solution). The solution is in every case to be freshly prepared immediately before using.

4. Dilute cresol water (2.5 per cent. ${ }^{1}$ ). This is prepared by adding to 50 c.c. [12 drams] of liquor cresolis compositus sufficient water to make 1 liter [1 quart] and mixing thoroughly.

5. Carbolic acid solution (about 3 per cent.). This is prepared by adding to 30 c.c. [ 1 ounce] of liquefied carbolic acid (phenol liquefactum) sufficient water to make one liter [1 quart] of disinfectant fluid, and mixing thoroughly.

6. Cresol-sulphuric acid solution. In the preparation of the solution, 2 parts by volume of crude cresol are mixed at ordinary temperature with 1 part by volume of crude sulphuric acid and the mixture is allowed to stand for at least 24 hours. Then for each 30 c.c. [ounce] of the mixture sufficient water is added to make a liter [quart] of disinfectant fluid, and thoroughly mixed. The solution must be used within 3 months.

When the solution is to be used to disinfect places in the open (courtyards, stock-yards, etc.) in cold weather, sodium chloride (0.5 to 1 kilogram [1.3 to 2.6 pounds] to each 10 liters [10 quarts]) may be added to prevent freezing, being thoroughly mixed in by stirring.

Stables, yards, utensils, etc.; which have been cleansed with soda or soap golution must be thoroughly washed with water to remove the remaining soda or soap before the cresol-sulphuric acid solution is applied.

7. Corrosive sublimate solution ( $1 / 10$ per cent.). Thissolution is prepared by dissolving in each liter [quart] of water 1 gram [15 grains] of corrosive sublimate and 1 gram [15 grains] of sodium chloride with a small amount of red coloring matter, or colored tablets, containing 1 gram [15 grains] of sublimate, may be used.

Stables, yards, utensils, etc., which have been cleansed with soda or soap solution must be washed with water to remove any remaining soda or soap before being disinfected with the sublimate solution. Disinfections in which large quantities of sublimate solution are used, as the disinfection of stables, yards, etc., can only be done under veterinary or police supervision. In disinfecting with sublimate solution, especially in disinfecting cattle stables, it is recommended that the disinfected surfaces and objects be washed after 24 hours with a $1 / 2$ per cent. solution of sulphurated potassium.

8. Formaldehyde solution (about 1 per cent.). This is prepared by adding to each 30 c.c. [ounce] of commercial formaldehyde solution (formalin) sufficient water to make 1 liter [quart] of the disinfectant solution, and mixing thoroughly.

${ }^{1}$ In swine plague and hog cholera a 6 per cent. cresol water is used. Instead of 50 c.c. [12 drams] of liquor cresolis compositus, 120 c.c. [4 ounces] are required for each liter [quart]. 
9. Steam in apparatuses which have been examined by experts when erected and at regular intervals afterward and found satisfactory.

In addition, steam from a steam boiler may be employed for small vessels with one opening, like milk cans, provided the steam is used under pressure and is introduced directly into the vessel. The interior of the vessel is first exposed to the live steam and then the hoops, bands and external wall, the latter especially in wood vessels.

10. Boiling in water or in 3 per cent. soda or soap solution (see $\$ 5$, No. 8). The fluid must be put on the fire cold, the objects completely immersed and the boiling continued for at least a quarter hour. The vessel must be covered.

Milk buckets, milk cans and other milk vessels, instead of being subjected to the boiling process described, may be treated in the following manner:

(a) The vessel is laid in boiling-hot water or in boiling-hot soda solution or dilute milk of lime for at least 2 minutes, all parts of the vessel being covered by the fluid.

(b) The external and internal surface of the vessels, the handles and covers are scrubbed with boiling-hot water or boiling-hot soda solution.

11. Thorough singeing and heating with fire or a suitable flame

12. Burning.

(Subdivision 2.) The disinfectants described in Nos. 4 to 7 are to be used as hot as possible.

(Subdivision 3.) According to further regulations of the government, other disinfectants and methods, which have been tested in regard to their effectiveness and practical application, may be employed in addition to those mentioned above.

\section{Choice of Disinfectant and Method of Application.}

$\$ 12$. The choice of the disinfectant $(\$ 11)$ and the method of application will depend in general upon the resistance of the infectious agent, the facility with which it is carried by intermediate bodies and the special conditions existing in each case.

§ 13. In epizoötics, the infectious agent of which is easily destroyed and which is spread by the diseased animals, it will be sufficient to clean and then whitewash with dilute milk of lime or calcium chloride solution the ceiling, walls, posts, pillars, stalls, doors, floors, gutters and equipment. Iron parts are to be wiped with dilute cresol water or carbolic acid solution. Wood and stone and glazed tiles are to be treated in the same manner.

§14. (Subdivision 1.) In epizoötics of which the infectious agent is difficult to destroy, or when there is great danger that the disease may be further spread by intermediate objects, the following process is to be followed:

1. The bedding, manure, other dirt, feed residue, etc., removed in clean- 
ing is to be collected together and either burned, buried, plowed under, or rendered harmless by composting or by mixing with a suitable disinfectant.

The compost piles containing the manure, straw, feed residue and similar material should be placed so that susceptible animals and unauthorized persons cannot walk over them, dirty fluids flow from them to other premises, strange persons have access to them, or washings from them contaminate springs, water courses or other sources of water supply. The materials should be thoroughly mixed in the proportion of two parts of manure to three of straw, moderately moistened, and loosely piled in large heaps for three weeke. After being piled in a heap, the manure is moistened with water or urine (about 10 to 15 quarts to 1 square yard of manure). The remainder of the process is as follows: A layer of non-infected manure, straw or turf about 10 inches deep, 5 to 6 feet broad and as long as desired is first placed upon the ground and upon this the manure to be disinfected is piled in a heap 4 feet high with sloping sides. The surface of the heap is covered with a layer of non-infected manure, straw, leaves, turf or other loose material four inches deep, and over this is placed a layer of earth of the same thickness. After remaining three weeks in this condition the manure may be removed without danger.

Manure and bedding material which has not been composted and which is required to be rendered harmless (see $\$ 15$ to 27 ) must be removed from infected premises in tight wagons without the employment of susceptible animals from other premises. In case of necessity, the manure, bedding material, etc., must be wet down in layers with concentrated milk of lime, unless the character of the infection requires the use of another disinfectant.

When the method of storing the manure which is permitted is such that there is danger of the infectious material being spread by contaminated water flowing into other premises, by the manure being accessible to strange persons or animals, or by springs, streams or other water supplies being contaminated, then the manure is to be treated with concentrated milk of lime in the stalls before it is removed to the place of storage.

2. Liquid manure and dirty water, in so far as they are not used in the composting of manure (No. 1), are to be disinfected with lime, concentrated milk of lime, calcium chloride or concentrated solution of calcium chloride. At least 1 part by volume of lime or calcium chloride or 3 parts by volume of concentrated milk of lime or concentrated calcium chloride solution are to be used to each 100 parts of liquid manure or dirty water. The mixture must be thoroughly stirred and allowed to stand for at least 2 hours.

3. Feed and straw stored in the rooms to be disinfected are to be removed without harm in so far as the regulations do not provide that they be disposed of in another manner ( $\$ 115$ to 27 ). 
4. Ceilings and walls, fittings (mangers, troughs, pillars, posts, stalls, doors, door posts, windows, etc.), floors, gutters, canals, troughs and pits are to be whitewashed with dilute milk of line or calcium chloride solution or brushed or thoroughly sprayed with dilute cresol water, carbolic acid, formaldehyde, corrosive sublimate or cresol-sulphuric acid solution.

Iron parts are to be treated with dilute cresol water or with carbolic acid solution.

5. Impervious paving or floors in court yards, railroad stock pens, slaughter houses, stock-yards, roads (streets), ships and ferries are to be washed or sprayed in a suitable manner with dilute milk of lime or calcium chloride solution or other disinfectants ( $\$ 15$ to 27 ). In cold weather the pavements are washed with cresol-sulphuric acid solution containing sodium chloride or are sprinkled with powdered, freshly-slaked lime.

The same process may be used for court yards, stock-yards, roads, streets and farm stock pens which have a paving that is pervious or which are unpaved.

6. Earth and sand floors which are not moistened with the excretions of diseased or suspected animals, including earth and sand under floors removed according to $\S 5,4$ to 7 , and the manure piles in sheep and cattle stables which are not removed in cleaning are to be sprayed with concentrated milk of lime or sprinkled with freshly slaked lime until the ground and manure are covered with a thick layer of lime.

7. Wood utensils, including vehicles and sleds used to haul cadavers, cadaver parts, straw, manure and the contents of the stomach and intestines of animals which have been killed or slaughtered, are, in so far as they cannot be burned, to be singed, or washed with dilute cresol water, carbolic acid, formaldehyde, cresol-sulphuric acid or corrosive sublimate solution.

8. Utensils of iron and other metals are to be exposed to the action of fire for a short time or washed with dilute cresol water, carbolic acid or formaldehyde solution.

9. Articles of leather, especially foot-gear, and rubber are to be carefully and repeatedly wiped with cloths which have been saturated with cresol water, carbolic acid or corrosive sublimate solution.

10. Linen, hemp (jute), cotton and woolen articles, clothing and bed covers, hair, wool, feathers, féed sacks, cushion stuffing, etc., which cannot be burned, or which are not required to be disposed of in another manner in certain diseases (see $\S \S 15$ to 27 ), are to be laid for 24 hours in dilute cresol water, carbolic acid, corrosive sublimate, or formaldehyde solution, or disinfected by boiling or in a steam apparatus.

Pieces of clothing which are only slightly soiled may be disinfected by moistening and scrubbing with dilute cresol water, carbolic acid, corrosive sublimate or formaldehyde solution.

11. Animals are to be washed with a proper disinfectant (see $\$ \S 15$ to 27), 
especially when in places in which the skin and hoofs or claws may have been soiled with manure or other excretions.

12. Hands and other parts of persons are to be thoroughly brushed with dilute cresol water, carbolic acid or corrosive sublimate solution and after about five minutes washed with warm water and soap.

(Subdivision 2.) The government may permit deviations from the processes described in Nos. 1 to 12 .

\section{Methods Prescribed for the Different Diseases}

\section{Anthrax}

\$15. 1. Persons who have come in contact with the bloody excretions. from an infected or suspected animal, or who have assisted in bloody operations on such animals or in the slaughtering or destruction of the same, or who have assisted in the opening or removal of the cadavers, shall immediately wash and disinfect thoroughly the hands and other parts of the body which may have been soiled, also soiled clothing and foot-gear.

2. As soon as an infected or suspected animal dies, is killed, recovers or is removed from its stall, the cleaning and disinfection must be immediately begun. This must include the stall in which the animal stood, the place where it died or was killed, and, where the disease is of frequent occurrence, certain parts of the stable or the entire stable, as the official veterinarian may in his judgment determine; also floors, walls, posts, pillars, stall divisions, mangers, racks, troughs, etc., stable and slaughtering utensils, clothing and foot-gear of the attendants and other objects which are contaminated with eliminations, blood or offal of infected or suspected animals or which may be assumed to be otherwise infected with the organisms of anthrax; stored feed or straw which diseased or suspected animals may have come in contact with or which may be assumed to have been otherwise infected; vehicles and vessels used to remove cadavers or parts of the same, offal, manure, etc., and, when necessary, contaminated places in the pasture, burial and storage places and water troughs.

3. The disinfection is earried out as directed in $\$ 14$ and the cleaning is preceded by a preliminary disinfection (see $\S 5$, No. $10 ; \S 6$, subdivision 2 ). As disinfectants, calcium chloride, dilute and concentrated calcium chloride solutions, corrosive sublimate or formaldehyde solutions are to be used. Special attention should be given to the solids and fluids liberated in killing, especially the bloody eliminations from diseased or suspected animals and their cadavers and blood. This material, together with the bedding, feed residue, manure, the earth removed from unpaved floors and articles of little value, is to be disposed of like the cadavers (see the directions for the harmless removal of the cadavers). Liquid manure contaminated with blood or bloody eliminations from diseased or suspected animals is to be disinfected with calcium chloride or solution of calcium chloride ( $\$ 14$, subdivision 1, No. 2). 
4. Stores of feed or straw which contain anthrax organisms, or which there is reason to believe may be infected, may be disinfected by steam in suitable apparatus or by exposure to sufficient heat through other methods. If this is not possible, the feed and straw is to be burned or buried, unless permission is given by the sanitary authorities to use it for animals which have undergone vaccination for anthrax.

\section{Black Leg and Hemorrhagic Septicamia}

$\S 16$. For black leg and hemorrhagic septicæmia the methods described in $\S 15$ are employed, except the preliminary disinfection specified in No. 3 .

\section{Rabies}

\$17. 1. Immediately after the death or destruction of a diseased or suspected animal, the place occupied by it, especially the floors, walls, mangers, racks, troughs, partitions, posts, pillars, and stalls contaminated by it, and the utensils and other objects with which it has come in contact, shall be cleaned and disinfected as directed in $\$ 13$.

2. In rabies of the dog and cat, the bedding, muzzle, collar, leash, blanket, utensils and other objects which have been used by the diseased or suspected animal shall be burned or rendered harmless in some other way. Dog houses of wood, straw, reeds or similar material are to be burned; those of other material are to be cleaned and disinfected as directed in $\S 13$.

\section{Glanders}

818. 1. Persons who have come in contact with diseased or suspected animals, cadavers or cadaver parts, are to immediately clean and disinfect their hands. Water, soap and suitable disinfectants (dilute cresol water, carbolic acid or corrosive sublimate solution) are to be at hand for this purpose in infected premises.

2. As soon as an infected or suspected animal is removed from its stall, the latter and all equipment and utensils used for the diseased or suspected animal are to be immediately cleaned and disinfected, except in so far as the latter are to be used for other diseased animals.

3. Before the removal of the quarantine restrictions, certain parts of the stable or the entire stable, as in the judgment of the official veterinarian may be necessary, the utensils and equipment (mangers, racks, posts, pillars, stall partitions, buckets and other stable apparatus, tie ropes, halters, harness, saddles, cleaning implements, covers, housing, clothing and foot-gear of the attendants, wagon poles, chains, water troughs, shoeing places, etc.) and other objects which have been in contact with diseased or suspected animals, their eliminations, cadavers or cadaver parts are to be cleaned and disinfected; also, any contaminated places in the pastures. 
4. The disinfection is to be carried out according to $\$ 14$ and the cleaning is to be preceded by a preliminary disinfection. Any of the disinfectants mentioned in §11, subdivision 1, may be employed. Special attention must be given to objects contaminated with nasal discharge, secretions from cutaneous ulcers, manure and urine from diseased or suspected animals. Manure, straw, feed residue, etc., may be composted and the urine or other eliminations from diseased or suspected animals treated with a disinfectant.

\section{Foot-and-Mouth Disease}

19. 1. The attendants of diseased or suspected animals in infected premises, persons who have engaged in the slaughter or transportation of such animals or in the removal of the bedding or the plowing under of the manure, and other persons who have come in contact with suspected or diseased animals in infected premises or who have visited stables in which such animals have been placed, must, before leaving, change or clean and disinfect the clothing and foot-gear and also clean and disinfect the hands and other parts of the body which may be contaminated.

2. Utensils, vehicles, vessels and other articles which have come in contact with diseased or suspected animals or their eliminations and which must be used outside of the infected premises during the continuance of the disease are to be cleaned and disinfected before being removed from the infected premises. Milk cans which must be removed from the infected premises for use outside during the prevalence of the disease are to be disinfected, after being emptied, as directed in $\S 11$, subdivision 1, Nos. 9 and 10 .

3. When manure is removed from infected stables it is to be composted on the premises or in some other suitable place from which the infection cannot spread ( $\$ 14$, subdivision 1, No. 1 ), or, if this is impracticable, concentrated milk of lime is to be poured over it before it is removed from the stable.

4. Urine and manure from ruminants and swine can be removed from premises during the prevalence of the disease only under the most pressing circumstances and with the permission of the sanitary authorities. The material must not be hauled with cattle from other premises. The liquid manure must be removed in tight vessels and the wagons used to haul manure which has not been composted must also be tight. If the material is hauled over public roads and the roads cannot be closed while the hauling continues, then the manure is to be wet with concentrated milk of lime. Manure which was not composted before removal is to be immediately plowed under or composted. In the latter case, when the packing process is completed access of ruminants and swine should be prevented.

5. At the final disinfection, which is to be carried out as directed in $\$ 11$, the places occupied by diseased or suspected animals (stables, yards, exercisé yards, bull stalls, breeding stalls, shoeing places, etc., stock pens, stock-yards 
and roads); the places used to store manure, cadavers and cadaver parts; the watering trough and its surroundings; harness; wagon poles; utensils used in attending to the diseased or suspected animals (watering buckets, milk pails, milk stools, milk cans, manure forks, shovels, etc.); feed sacks; skin, horns, claws, wool and other animal products which may be infected either because of their origin or place of storage, and the clothing and footgear of the attendants are to be cleaned and disinfected. Special attention is to be given to objects which have been contaminated with saliva or manure from diseased or suspected animals. Skin, horns, claws and other raw animal products are to be disinfected by complete drying or by immersion for 24 hours in dilute milk of lime or through treatment with other disinfectants. Wool may be removed from the infected premises if it is packed in closed Eacks.

6. Feed and straw which have been stored in infected stables or which have been otherwise contaminated by the eliminations from diseased or suspected animals cannot be removed from the infected premises but must be used there or rendered harmless.

7. At the final disinfection, the claws of cattle from the infected stable are to be trimmed and the animals are to be cleaned and disinfected ( $\$ 4$ and $\$ 14$, subdivision 1 , No. 1 ).

8. Finally, at the final disinfection all of the attendants employed on the premises which were infected and all persons who otherwise came in contact with diseased or suspected animals are to clean and disinfect the hands and arms and any other parts of the body which may have come in contact with the animals.

9. Any of the disinfectants named in $\$ 11$, subdivision 1 , may be used.

\section{Lung Plague}

$\$ 20$. 1. The attendants who have cared for diseased or suspected animals, persons who have been engaged in the slaughter or transportation of such animals, and other persons who have come in contact with diseased or suspected animals or who visit stables in which such animals are kept, must, before leaving the infected premises or slaughter place, change the clothing and foot-gear or clean and disinfect the same, and must also clean and disinfect the hands and the other parts of the body which may have come in contact with the diseased animals.

2. During the prevalence of the disease on infected premises, in case of the removal of a diseased or suspected animal from its stall or from the stable, the stall and its fittings and the utensils used in caring for the animal must be at once cleaned, and disinfected in accordance with the directions in $\S 13$. Feed residue contaminated by the expired air of the animal must be burned or treated in the same manner as the manure and bedding. 
3. Manure and bedding must be removed from infected premises to the field and plowed under without the use of cattle from other premises. If it cannot be plowed under at once, it must be piled up in heaps and cattle must not be permitted to come in contact with it for at least 2 weeks.

4. At the final disinfection, the infected stable and other compartments on the infected premises which have been occupied by diseased or suspected animals or their cadavers, the equipment and utensils which have come in contact with such animals, and the clothing and foot-gear of the attendants are to be cleaned, and disinfected as directed in $\$ 13$.

5. Feed and straw stored in the infected stables or over such stables above a loose ceiling must not be removed from the premises after the disease has been stamped out. These materials may be used for horses, swine or sheep, but must be placed where cattle cannot come in contact with them. If they cannot be disposed of in this way, they are to be treated in the same manner as the manure.

\section{Sheep-pax}

§21. 1. The attendants who have cared for diseased or suspected sheep in infected premises, those persons who have been engaged in shearing, slaughtering or transporting such animals, and other persons who have come in contact with diseased or suspected animals or who visit stables in which such animals are kept, must, before leaving the infected premises, change or clean and disinfect their clothing and foot-gear and must also clean and disinfect the hands and other parts of the body which may have come in contact with the diseased animals.

2. The manure is to be permitted to remain in the stable until the final disinfection. If it is necessary to remove it, then it is to be composted ( $\$ 14$, subdivision 1, No. 1) on the premises or in some other suitable place from which the infectious material cannot spread; or, if this is not possible, it must be wet with concentrated milk of lime before removal. Manure which cannot be composted on the premises can only be removed with the permission of the sanitary authorities; it must be hauled to the field in tight wagons and immediately plowed under or composted. In the latter case, access of strange sheep must be prevented until the composting is finished.

3. At the final disinfection, the stables and other compartments in which diseased or suspected animals have been kept, the equipment and utensils which came in contact with such animals or their eliminations, and the clothing and foot-gear of the attendants are to be cleaned and disinfected. Any of the disinfectants named in $\$ 11$, subdivision 1 , may be used in the disinfection, which is to be carried out as directed in $\$ 14$.

4. Feed and straw stored in infected stables or over such stables above a loose ceiling are to be thoroughly aired and used only on the infected premises or rendered harmless. 


\section{Dourine and Vesicular Exanthema}

$\$ 22$. No disinfection is required for dourine and vesicular exanthema.

Mange [Scab]

\$23. 1. During the treatment of the diseased or suspected horses or sheep, or of sheep flocks in which the disease exists, the stables, hurdles, equipment, utensils, blankets, cleaning implements and other objects which have come in contact with such animals are to be cleaned and disinfected. When the animals are treated by dipping, the cleaning and disinfection is to be carried out at each dipping. If the animals are treated by hand applications, the cleaning and disinfection is to be repeated at shorter or longer intervals according to the degree of the disease. After the completion of the curative treatment, the final disinfection is to be made.

2. Stables or other compartments and hurdles used by horses or sheep affected with mange before slaughter, or before treatment was begun, must be immediately cleaned and disinfected after the diseased animals are removed.

3. The disinfection is carried out as directed in $\$ 14$. As disinfectants, dilute cresol water, carbolic acid solution or lime are to be used. Special attention is to be given to all objects with which the diseased or suspected animals have come in contact (mangers, racks, posts, pillars, stalls, tie ropes, halters, harness, saddles, cleaning instruments, blankets, housing, clothes of attendants, wagon poles, etc., in connection with horses; hurdles, racks, mangers, posts, sheep folds, sheep shears, shovels, manure, clothing and footgear of the attendants, etc., in connection with sheep).

4. The manure from the infected stables is to be removed to the freld and immediately plowed under. If the latter cannot be done at once, the sheep are to be kept away from the manure until the work is finished.

[The regulations of the U. S. Bureau of Animal Industry (B. A. I. order No. 143) regarding disinfection for cattle mange and sheep scab are as follows:

Regulation 28. Cars and other vehicles, yards, pens, sheds, chutes, etc., which have contained diseased cattle shall be cleaned and disinfected in the following manner: Remove all the litter and manure and then saturate the interior surfaces of the cars and woodwork, flooring, and ground of the chutes, alleys, and pens with a 5 per cent. solution of pure carbolic acid in water, with sufficient liwe to show where it has been applied.

Regulation 37. Cars and other vehicles, yards, pens, sheds, chutes, etc., that have contained diseased sheep shall be cleaned and disinfected in the following manner: Remove all litter and manure and then saturate the interior surfaces of the cars and the woodwork, flooring, and ground of the sheds, alleyways, and pens with a solution containing 5 per cent. of pure 
carbolic acid or with a solution containing 2 per cent. of cresol. When cresol is used it must be mixed with soft soap in order to render it easily soluble in cold water.]

\section{Swine Plague and Hog Cholera}

824. 1. Manure, bedding, feed residue, etc., remored from infected pens are to be composted ( $(14$, subdivision 1, No. 1 ). If this is not feasible, then these materials are to be burned or buried with the cadavers of animals which die or are destroyed. The rejected parts of slaughtered diseased animals are also to be buried or burned and the offal, including the water used to wash the meat and viscera, is to be rendered harmless.

2. The passageway between the pens, the place before the stable door, the entrance to the yard and the roads to the stable and on the farm are to be cleaned and disinfected with dilute calcium chloride solution or 6 per cent. cresol water at least every 8 days during the continuance of the disease.

3. Utensils, vehicles, vessels and other articles which have come in contact with diseased or suspected animals or with their eliminations are to be disinfected before being removed from the premises.

4. At the final disinfection the places in which the diseased or suspected animals have been kept (pens and adjoining compartments such as the feedcooking room, exercise place, yard, breeding pen, stock-yards, railroad stock pens, etc.), the utensils (buckets, forks, shovels, troughs, etc.) used in caring for and in slaughtering such animals, the vehicles and sleds used to haul the cadavers, straw, manure and other offal, the clothing and foot-gear of the attendants, feed sacks and other articles which have come in contact with diseased or suspected animals or which there is reason to assume may be infected, are to be cleaned and disinfected. Special attention is to be given to objects contaminated with the manure, urine and blood of diseased or suspected animals. The manureand straw are to be removed from the rootingplace and the superficial layer of earth is to be removed, after which, if possible, the surface of the ground is to be thoroughly saturated with dilute calcium chloride solution or with 6 per cent. cresol water, then raked or harrowed until level and repeatedly saturated with the disinfectants named. New swine brought on to the premises are to be kept away from the rootingplace as long as possible. Earth removed from the floors and other places is to be removed to fields not accessible to swine and buried or plowed under.

5. The disinfection of the pens and other compartments used by diseased or suspected animals is to be carried out according to the directions in $\S 14$. As disinfectants, dilute calcium chloride solution or 6 per cent. cresol water are to be used.

[The regulations of the U.S. Bureau of Animal Industry (B. A.I. order 143) contain the following regarding disinfection for hog cholera and swine plague: 
Regulation 45. Cars and other vehicles and pens or yards which have contained interstate shipments of diseased or exposed swine shall be cleaned and disinfected as soon as possible after unloading. Cars that have contained interstate shipments of swine shall not be removed until the inspector has ascertained the condition of the live animals and either released the cars or given notice that they shall be cleaned and disinfected. Cleaning and disinfection shall be done by first removing all litter and manure and then saturating the interior surfaces of the cars and the woodwork, flooring, and ground of the chutes, alleys, and pens with a 5 per cent. solution of pure carbolic acid in water, or with a solution containing 2 per cent. of cresol. When cresol is used it must be mixed with soft soap in order to render it easily soluble in water. (Subsequently, it was found that a 3 per cent. solution of the official liquor cresolis compositus was more efficient than the carbolic acid solution.)]

\section{Srine Erysipelas}

$\$ 25$. 1. When swine erysipelas is present, the cleaning and disinfection includes, as a rule, the pen occupied by the diseased or suspected animal; when several cases occur a certain portion of the building or the entire building occupied by swine is to be disinfected, as in the judgment of the official veterinarian may be necessary; also, the equipment, utensils and other objects which have come in contact with diseased or suspected animals or their excretions, cadavers or cadaver parts. The pen occupied by the diseased or suspected animals, a portion of the building or the entire building, as may be required, is to be cleaned and disinfected immediately after the death, slaughter or removal of all the swine, or within 6 days after the last case of the disease.

2. The disinfection is to be made as directed in $\S 13$. Any of the disinfectants named in $\S 11$, subdivision 1 , may be employed.

\section{Fowl Cholera and Fowl Pest}

$\$ 26$. 1. The coops and other compartments occupied by diseased or suspected fowl, the cages, other containers and transportation crates, the run, baths or swimming pools, the utensils and other objects which have come in contact with such fowl, their excretions, cadavers or cadaver parts, and the places where such fowl have been slaughtered are to be cleaned and disinfected as prescribed in §14. Any of the disinfectants named in §11, subdivision 1, may be employed.

2. Special attention must be given to the eliminations of diseased or suspected fowl and to blood escaping at the slaughter of the same. This offal is to be carefully collected and buried or burned with the cadavers, together with the litter, manure, feathers, feed residue, earth removed from unpared floors and from the run, and all other objects of slight value which are contaminated with manure or blood. Rejected parts of slaughtered, diseased or 
suspected fowl and other slaughter offal are to be rendered harmless by being burned or buried. Offal of such a nature that it cannot be burned must be collected together and thoroughly mixed with an equal amount of mill of lime and then buried. The surface layer of earth is to be removed from the runs and the ground is then to be wet with concentrated milk of lime. Iime or concentrated milk of lime is to be mixed with the water in the swimming pools or baths in the same manner as it is mixed with liquid manure ( $\$ 14$, No. 2, subdivision 1 ); after 24 hours the water is to be removed and the sides and bottom of the pool or bath disinfected with concentrated milk of lime.

3. Large quantities of manure may be composted. Feathers in dry condition may be removed from infected premises packed in closed sacks, with the approval of the sanitary authorities.

\section{Tuberculosis}

\$27. 1. Immediately after cattle in which tuberculosis is established according to law or in which its presence appears probable are removed, the stalls occupied by them are to be cleaned and disinfected.

2. The cleaning and disinfection, as a rule, includes the stall, but in the event of the occurrence of several cases, or if the diseased or suspected animal has occupied different stalls in the stable, then the official veterinarian may require the eleaning and disinfection of a portion of the stable or the entire stable, as he may consider necessary. In every case the equipment, utensils and other objects contaminated by the elimination of the animal are to be cleaned and disinfected, especially the manger, feed vessels, racks, water troughs, the alleys and walls adjoining the stall, the floor, and the cleaning and milking utensils.

3 . The disinfection is to be carried out according to $\S 13$. Milk pails and other milk vessels are to be disinfected by steam, by boiling or by scrubbing with boiling hot water or boiling hot soda solution (see §11, subdivision 1, Nos. 9 and 10 ).

\section{[Southern Cattle Fever}

The regulations of the U. S. Bureau of Animal Industry (B. A. I. order 143, amendment 4) require that cars which have carried cattle from the quarantined area shall be cleaned and disinfected as follows: "Remove all litter and manure from all portions of the cars, including the ledges and framework outside; wash the exterior and interior of the car until clean and saturate the entire interior surface, including the inner surface of the car doors, with the disinfecting material."

Boats which have carried cattle from the quarantined area must be cleaned and disinfected as follows: "Remove all litter and manure from the decks, stalls, and all other parts of the boat occupied or traversed by such cattle, and from the portable chutes or other appliances or fixtures used in loading 
and unloading same, and wash them until clean, and saturate the entire surface of the decks, stalls, or other parts of the boat occupied or traversed by the cattle, or with which they may have come in contact or which have contained litter or manure, with the disinfecting material."

Yards, pens, chutes, and alleyways used by cattle from the quarantined area must be cleaned and disinfected as follows: "Empty all troughs, racks, or other feeding or watering facilities and wash them until clean; remove all litter and manure from the floors, posts, or other parts and wash them until clean, and saturate the entire surface of the fencing, troughs, chutes, floors, walls, and other parts with the disinfecting material.

"As materials for the disinfection of cars, boats, pens, chutes, and alleyways which have contained cattle of the quarantined area one of those indicated below shall be used. (1) A mixture made with not more than 11/2 pounds of lime and $1 / 4$ pound of pure carbolic acid to each gallon of water. In lieu of the pure carbolic acid required to make this solution a proper quantity of socalled 'crude carbolic acid' of known strength (but not less than 25 per cent. pure) may be used, sufficient to make a disinfecting solution containing 5 per cent. of the pure acid. (2) Any coal-tar creosote dip permitted in the official dipping of sheep for scabies, provided the same is used at one-fifth the maximum dilution (five times the minimum strength) specified for dipping sheep.

"The litter and manure removed from cars, boats, or other vehicles, and from pens, chutes, alleyways, or other premises or inclosures which have contained cattle of the quarantined area, shall not be so located or stored that they come in contact with cattle in course of interstate transportation unless disinfected by one of the methods specified below. (1) It may be disinfected by saturating it with any disinfecting material specified in the preceding paragraph of the strength and composition indicated therein, except that the lime may be omitted. (2) It may be stored without disinfection during the period from February 1 to October 31, inclusive, of each year; when stored as above indicated, the storage space shall be tightly inclosed and so situated or so surrounded by cattle-proof fences or other structures that no cattle other than cattle of the quarantined area may approach closer to it than 15 feet."]

\section{THE MOST IMPORTANT DISINFECTANTS FOR ANIMAL} INFECTIONS

Calx.-Lime. Freshly slaked lime (caustic lime) is a good and easily prepared disinfectant for spore-free bacteria. It is also the cheapest disinfectant. Anthrax spores and tubercle bacilli are, however, not killed by lime. It is used in the form of a powder, 
concentrated milk of lime $(1: 3)$ and dilute milk of lime $(1: 20)$, the concentrated solution being applied as a whitewash, and the dilute solution being used to disinfect the floors and also as an addition to manure and urine. A single application of dilute milk of lime $(1: 20)$ kills the bacteria of fowl cholera, a triple application ( $1: 20$ ) destroys the bacilli of glanders; a single application of concentrated milk of lime $(1: 3)$ kills the bacilli of swine erysipelas, the hog cholera bacillus, the anthrax bacilli and typhoid fever bacilli. Lime operates only in its free state as a caustic; it loses its disinfectant properties as soon as it combines with acids; the carbonate, phosphate, sulphate and nitrate of lime are ineffective. It must be added to the substances to be disinfected (manure, urine) in sufficient quantity to make the reaction of the mixture strongly alkaline, because, under certain conditions, the growth of microörganisms is facilitated if the reaction of an acid substance is rendered neutral or only weakly alkaline. Since the preparation of milk of lime from burned lime is somewhat difficult and complicated, lime from lime pits may be used, but the upper layer should first be removed.

Special consideration has been given to the disinfection of manure from slaughter houses and cattle yards with lime, especially the composting of manure with and without lime. With the exception of anthrax, black leg, glanders, rinderpest and rabies, in which it is best to burn the manure, in all other cattle infections the manure can be certainly, simply and cheaply disinfected with a fresh, dilute milk of lime and without injuring its agricultural value. In composting without lime, a temperature sufficiently high $\left(60\right.$ to $70^{\circ} \mathrm{C}$.) is developed to exert a disinfectant action upon the organisms of swine erysipelas, fowl cholera, - glanders, contagious pneumonia, strangles, tuberculosis, hemorrhagic septicæmia, etc., provided care is taken to have the manure moderately moist (straw: manure-3:2), piled rather loosely and covered with material that conducts heat poorly (Pfeiler, Bohtz).

Calx chlorinata. Chloride of lime. This is more powerful than lime; in a solution of $1: 3$ it kills even anthrax spores. It is therefore superior to lime for whitewashing walls, washing floors, 
ete., in infectious outbreaks; also, for the destruction of infectious material difficult to destroy, especially as a cheap disinfectant for anthrax spores and for the disinfection of urine. According to Hansen (Monatshefte für prak. Tierheilkunde, 1912) the addition of 0.2 per cent. of calcium chloride to liquid manure is a certain and cheap disinfectant and is not harmful to vegetation and does not destroy the fertilizing value. Chloride of lime is uncertain against glanders and tuberculosis. A 1 per cent. solution kills the bacteria of fowl cholera, swine erysipelas and hog cholera and the bacilli of anthrax in one minute. Freund recommends the use of a $\mathbf{5}$ per cent. filtered solution of chloride of lime for the disinfection of railroad cars; the process is very complicated (spraying with a pumping apparatus under $1 \frac{1}{2}$ atmospheres pressure). The formerly much used chlorine gas, in the presence of sufficient moist air and in strong concentration ( 1 per cent. by volume), will destroy all air-dried microörganisms within 24 hours, but for practical disinfection it is of no value because the necessary concentration is difficult to obtain and is very costly. The process of chlorine disinfection is also very complicated and dangerous. The same applies to bromine.

Lyes. The antiseptic effect of the lyes was formerly overvalued; their disinfection power is about equal to lime. Soda lye, potash lye, soda and potash are in general of the same value. Ammonia, on the other hand, is three to five times weaker. Concentrated solutions of these substances kill the bacteria of fowl cholera and swine erysipelas and glanders and anthrax bacilli. Anthrax spores and tubercle bacilli, on the other hand, remain intact. An objection is the caustic action of solutions of the required concentration. Boiling hot soda solution possesses a very high disinfection power.

Soaps. The disinfectant value of soaps depends upon their alkali content; the ordinary soft soap is therefore the most powerful. The action is increased by using boiling-hot soap water. The latter kills the more readily destroyed infectious agents and at the same time has a cleansing effect which assists the action of the stronger antiseptics. A 10 per cent. solution of the ordinary 
soft soap (or of ordinary washing lye) will kill anthrax spores in 10 minutes if heated to $80^{\circ} \mathrm{C}$. Heating the soap solution (and the soda solution) to $50^{\circ} \mathrm{C}$. is not sufficient.

Acids. They are in general poor antiseptics, but are colyseptics (inhibit development of bacteria). Sulphuric acid does not kill anthrax spores in 1 per cent. solution even after 20 days" action; its irritant effect upon the skin and mucous membranes is also objectionable. The experiments with acid turf litter (footand-mouth disease) therefore proved unsatisfactory. On the other hand, a combination of hydrochloric acid ( 2 per cent.) with sodium chloride (10 per cent.) is a strong disinfectant; this "pickling fluid" disinfects anthrax and black leg hides with certainty.

Hydrargyri chloridum corrosivum. Corrosive sublimate. The strongest and quickest disinfectant for all bacteria, especially all spore-forming microörganisms (anthrax spores), with the exception of tubercle bacilli; odorless, very cheap and easily and conveniently transported (sublimate tablets). Anthrax spores are destroyed with certainty and quickly only by sublimate. A 1:1000 solution destroys all of the organisms of the infectious diseases, including anthrax spores. The disinfectant action is increased by the use of warm solutions. The decomposition of the sublimate by ordinary water may be prevented by the addition of sodium chloride (sublimate tablets) or organic acids. When the disinfection is done carefully, the poisonous properties of sublimateare of no consideration, not even in the disinfection of cattlestables (subsequent washing with $1 / 2$ per cent. solution with sulphuretted potash).

Phenol. Carbolic acid. A weak disinfectant (100 times weaker than sublimate, 10 times weaker than creolin, lysol, etc.); ineffective against anthrax spores. On the other hand, the glanders bacillus and the less resistant bacteria (anthrax bacilli, etc.) are quickly killed by a 2 per cent. solution. A 5 per cent. solution is necessary to destroy tubercle bacilli. The odor of carbolic acid is unpleasant and is transmitted to the meat and milk (poisoning of man). Carbolic acid disinfection is also relatively expensive.

Cresol. ( ${ }^{*}$ Creolin, *lysol, *bacillol.) A very effective and cheap disinfectant; far superior to carbolic acid; effective against 
anthrax spores and rabies virus. Anthrax bacilli are destroyed by a solution of $1: 5000$. Used in 1 to 3 per cent. solution in water. For extensive disinfection, especially of railroad cars, Fischer and Koske (Arb. aus d. Kais. Gesundheitsamt, 1903) recommend a 3 per cent. solution in water of a mixture of 2 parts of cresol and 1 part of sulphuric acid as more effective and cheaper than *lysol or *liquor cresolis saponatus. Cresol-sulphuric acid solution to which sodium chloride is added only freezes when the temperature falls to 17 to $8.6^{\circ} \mathrm{F}$., which makes it especially suitable for winter disinfection (Kraut, ibid., 1907).

Pix liquida. Tar. An excellent disinfectant covering. Wood tar kills all pathogenic microörganisms, including anthrax spores and tubercle bacilli. Coal tar destroys with certainty the bacilli of anthrax, glanders and swine erysipelas, etc.

Liquor formaldehydi. Formaldehyde is recommended as a gaseous disinfectant for closed compartments, especially railroad cars; very high disinfectant power. A certain disinfectant action, however, can only be obtained by the employment of complicated apparatus (lamps). On account of the volatility of formaldehyde, the compartment to be disinfected must be made air tight (impossible with cattle cars). Furthermore, the disinfectant action is only superficial, and anthrax spores are not destroyed. The process is very expensive (a compartment of 100 cubic metres requires the action of 500 grams of formaldehyde, or $1 \frac{1}{2}$ liters of the official solution for $31 / 2$ hours). Disinfection with fluids, 2 to $21 / 4$ per cent. of formaldehyde, has the same disadvantages and is also injurious to health. See the investigations of von Perkuhn concerning the disinfection of stables with formaldehyde vapor by means of Lingner's apparatus (Monatshefte für prakt. Tierheilkunde, 1905).

*Autan. This preparation, which is used in a new method of formaldehyde disinfection, is in the form of a dry powder containing a quantity of formaldehyde with alkaline peroxides; formaldehyde vapor is liberated when water is added. Contradictory results have been obtained in the investigations of this new material and the experiments are not yet concluded (a newer and, it is alleged, an improved preparation). The same conditions are 
necessary for autan as for formaldehyde (see above), especially the complete sealing of the compartment, and a temperature not under $10^{\circ} \mathrm{C}$. is also required (not always possible with railroad cars). In addition, autan is also expensive. According to Löfler (Zeitschr. f. Vetkde., 1909), the organisms of swine erysipelas, hog cholera, strangles, fowl cholera, anthrax and tuberculosis are readily destroyed by autan. The disinfection must continue 7 hours, the compartment must be completely sealed and litter and manure must be removed.

Chinosol. This preparation is very active in inhibiting the development of bacteria, but has only a slight antiseptic action. It is ineffective against anthrax spores; very expensive, gives an unpleasant odor to the animal body, is not absolutely non-toxic and cannot be used for the disinfection of iron objects, manure, etc., because it is decomposed by iron and alkaline substances.

HEAT.-1. A glowing heat acts the strongest (disinfection of chains and other iron objects).

2. Burning is the best method of rendering anthrax and black leg cadavers harmless; a burning process which has proven satisfactory in practice has been described by Lothes and Profe (Fortschritte der Hygiene, 1904).

3. The disinfectant action of dry heat is generally overrated. Spore-free bacteria are killed by dry heat of $100^{\circ} \mathrm{C}$. In the usual method of employing dry heat (oven), however, $100^{\circ} \mathrm{C}$. of heat does not penetrate the object of disinfection because dry air is a poor conductor of heat. Spore-containing bacteria (anthrax spores) require much higher degrees of heat $\left(140^{\circ} \mathrm{C}\right.$.) operating for hours. The spores contain an almost water-free albumin, which it is very difficult for the dry heat to penetrate. With the employment of the hot air sterilizer (oven, $140^{\circ} \mathrm{C}$.), the heat in the interior of the object to be disinfected is usually insufficient. After 3 hours' exposure to $140^{\circ} \mathrm{C}$. in a hot air sterilizer, a woolcovered ball showed a temperature of only $35^{\circ} \mathrm{C}$. in the centre.

4. Boiling water kills spore-free bacteria in a short time (boiling the milk in foot-and-mouth disease). Heating milk for 20 minutes at $65^{\circ} \mathrm{C}$. kills tubercle bacilli, colon bacilli, streptococci 
and staphylococci (pasteurization). Anthrax spores withstand boiling temperature. Since the heat only penetrates large, solid objects slowly, it is recommended that the boiling be continued for at least one hour. Boiling water is not a certain disinfectant when used in the disinfection of stables because it cools rapidly when spread out on cold surfaces. In disinfecting by boiling, the water containing the object to be disinfected is placed upon the fire cold and is allowed to remain for at least $1 / 4$ hour after boiling begins; the vessel is to be covered. The German veterinary sanitary laws require that when foot-and-mouth disease is present milk shall be disinfected by boiling, and that in the case of tuberculosis it shall be sufficiently heated, i.e., heated over an open fire until it repeatedly boils, exposed to live steam at $85^{\circ} \mathrm{C}$., or heated in a water bath at $85^{\circ} \mathrm{C}$. for a minute or at $70^{\circ} \mathrm{C}$. for a half hour. The action of hot fluids is especially effective when a disinfectant is added.

5. Live steam of a temperature of $100^{\circ} \mathrm{C}$. is the most effective form of disinfection by heat (sterilizing apparatus) and should therefore be preferred, when convenient, to boiling; has not only a superficial but also a deep action. Anthrax spores and tubercle bacilli are killed in 5 minutes. The best results are obtained with an atmospheric pressure of about one-tenth. It is important that the temperature be not permitted to fall below $100^{\circ} \mathrm{C}$., and that an abundant supply of steam be introduced into the apparatus in order that the air (poor heat conductor) will be expressed as completely as possible (apparatuses of Henneberg, Rohrbeck, Lautenschläger, etc.). Disinfection with steam is most suitable for blankets and clothing; on the contrary, leather articles (saddles, girths, halters) are spoiled. Stables, railroad cars and large compartments in general cannot be disinfected with steam on account of its rapid cooling. In using steam on railroad cars, it was found that the highest temperature of the steam at the outlet tube was 90-95 ${ }^{\circ} \mathrm{C}$; s slightly removed from the outlet tube ( 4 inches) the temperature was only $60^{\circ} \mathrm{C}$, 40 inches distant $50^{\circ} \mathrm{C}$., 80 inches distant $20^{\circ} \mathrm{C}$., while 16 to 20 feet distant the temperature was raised only a few degrees (Redard and Colin). A temperature of $100^{\circ} \mathrm{C}$. cannot be attained in a stable even with steam from 
boilers carrying 8 atmospheres pressure (Kramell, Zeitschr. f. Vet., 1901). On the other hand, milk cans and other small vessels are surely disinfected with live steam under moderate pressure (Junack, Monatshefte f. prakt. Tierheilk., 1904). The addition of a slight quantity of a chemical disinfectant increases the effectiveness of steam considerably; hides and leather articles (saddles) which will only bear steam at $70^{\circ} \mathrm{C}$. are disinfected in a relatively short time by steam at $70^{\circ} \mathrm{C}$. containing 1 to 2 per cent. of formaldehyde, even anthrax spores being destroyed in 5 minutes (von Esmarch, Hygien. Rundschau, 12 Bd.).

Cold cannot be used for disinfection. Very low temperatures $\left(-100^{\circ} \mathrm{C}\right.$.) do not destroy anthrax spores. Even the bacteria of fowl cholera, which are rather easily destroyed, resist a temperature of $-4^{\circ} \mathrm{C}$.

Mechanical Disinfectants.-1. Burial is a very important method of disposing of infected carcasses, cadaver parts, manure, etc. (see the regulations for anthrax, rabies, glanders and lung plague). Bacteriological investigations have shown that the earth is free from bacteria at a depth of 5 feet, provided the excavation is not made in the neighborhood of houses, stables, manure pits, wells, etc., and does not reach the ground water. Most of the pathogenic bacteria, except anthrax spores and tubercle bacilli, die very soon in buried cadavers. Anthrax spores, however, retain their virulence many years and tubercle bacilli for several months (cholera and typhoid fever bacilli in human bodies die in 2 to 3 weeks after burial). Investigations have shown that the earth of cemeteries contains no more bacteria than the earth of cultivated fields at an equal depth. On account of the possibility of buried spores being disseminated (earth worms, moles), it is recommended that anthrax carcasses be buried in cemented pits.

2. Drying in the air has a very slight disinfectant action. Anthrax spores retain their virulence in dry places for years, and most of the more readily destroyed bacteria die only after several weeks.

3. Light assists the disinfectant action of the antiseptics. Spores appear to be more susceptible to light than the bacilli. Anthrax spores are killed by sunlight in 5 hours, while the bacilli 
are destroyed only after 30 hours (Arloing, Roux). Tubercle bacilli are killed by direct sunlight in a few hours and by diffuse daylight in a few days (R. Koch). The contagion of rinderpest is affected in the same manner (Theiler). Direct sunlight exerts a powerful disinfectant action upon coli bacilli and the organisms of swine plague, hog cholera, swine erysipelas and fowl cholera, while diffuse daylight also possesses disinfectant properties but in less degree (Neumark, Dissertation, 1907). Perhaps this explains the milder course and less frequent occurrence of some of the infectious diseases in the summer (contagious pneumonia). From the foregoing it follows that the admission of light into dark stables and the airing of blankets essentially assist disinfection.

Other mechanical methods include: whitewashing or painting (lime, calcium chloride, tar, varnish, paint, lacquer), which covers and fixes the bacteria and prevents their increase; scouring, cleansing, washing, sweeping, wiping, rubbing (rubbing the carpet with bread), exclusion of air (closing a room before disinfection) and above all washing with soap. The latter is sufficient in many cases, especially for infectious material that is readily destroyed.

\section{DISINFECTION OF WOUNDS}

The Most Important Antiseptics for Wounds.-The general statements already made (p. 170) are to be considered in connection with the choice and judgment of the individual antiseptics employed in the treatment of wounds. In the first place, attention is to be given to the different disinfectant values of the individual antiseptics and to the resistance of the microörganisms concerned in wound infection to these agents. As already mentioned on page 175, the resistance of these organisms lies about midway between that of the anthrax and black leg spores, which are diffcult to kill, and that of the easily destroyed anthrax and swine erysipelas bacilli. The staphylococci and streptococci especially cannot be destroyed by the milder disinfectants, but require the more powerful antiseptics (corrosive sublimate, creolin, lysol, tannoform, carbolic acid, tincture of iodine, aluminum acetate, silver nitrate). Other points to be considered are the degree of 
toxicity of the disinfectant, its irritant effect upon the wound, its decomposition by the wound secretions, the duration of its action upon the wound, the concentration and form, the price, and the stage of healing in the wound. In general, the rule to follow is to choose the most effective disinfectant which can be used in solution or in powder and which at the same time is the least irritant, the least liable to decomposition, the least poisonous and the cheapest; and to apply it to the wound as early as possible (tannoform!) and to permit it to operate as long as may be practicable. A thorough cleansing should precede the disinfection. The aseptic treatment of wounds has already been discussed on page 171 . In regard to the most important antiseptics for the treatment of wounds, the following may be mentioned:

Hydrargyri chloridum corrosivum. Corrosive sublimate. A powerful but poisonous antiseptic. A 1 to 1000 solution quickly destroys all microörganisms concerned in wound infection. It may be used as a wound disinfectant on all animals except ruminants. It is especially poisonous for cattle and it should not be used on these animals. In contact with albuminous wound secretions, corrosive sublimate is in part precipitated in the form of albuminate of mercury and in part decomposed (formation of mercuric oxychlorides). These decompositions, however, do not essentially affect the antiseptic action of the sublimate qualitatively; and, furthermore, they can be prevented by the addition of sodium chloride, hydrochloric acid or acetic acid. The advantages of corrosive sublimate are its strong disinfectant properties, its lack of odor, cheapness and convenience (sublimate tablets). Its disadvantages are its high toxicity, especially for cattle; its strong irritant action, especially upon the mucous membranes of the eye (ophthalmology) and uterus (obstetrics), and its rapid formation of an amalgam with instruments.

${ }^{*}$ Creolin, *lysol ${ }^{1}$ and other cresol compounds. The antiseptic action of cresol preparations is very powerful and rapid. A 3 per cent. solution destroys all of the organisms concerned in wound infection immediately. The disinfectant action of cresol is ten times

[1 The official liquor cresolis compositus is very similar.] 
stronger than that of carbolic acid; it possesses deodorizing propties and is relatively non-toxic. Its disadvantages are the odor, the irritant effect of strong solutions upon the mucous membranes and the partial turbidity of the solutions.

Phenol. Carbolic acid has a relatively good antiseptic action. A 3 per cent. solution kills most of the organisms concerned in wound infection after prolonged action. It is less effective, however, against tetanus bacilli, rabies virus, tubercle bacilli and anthrax spores. Castration clamps transmitted tetanus 18 months after they were infected, although they were immersed for five minutes in a 4 per cent. carbolic acid solution. The advantages of carbolic acid are that it does not decompose and is constant in its composition. The disadvantages are the odor, high price and the irritant and poisonous properties, the latter especially for cats.

Iodoformum. Iodoform. An excellent, mild antiseptic, which stimulates granulation; may be employed in the form of ether solution $(1: 5-10)$. The disadvantages are its odor, high price, toxicity for dogs (licking) and insolubility in water. Similar preparations, which are much more expensive and therefore not adapted to veterinary practice, are *loretin, sozoiodol, *losophan, *iodophen, *europhen, *aristol, *iodoformin, *iodoformogen, iodol, *iodine trichloride (very unstable), and others.

*Tannoform. The best remedy known at this time for forming aseptic scabs, and also a dry antiseptic. Applied early to fresh wounds, it prevents suppuration (healing under a scab). It is to be preferred to iodoform, especially for horses, on account of its stronger antiseptic action, lack of odor and lower cost. The other condensation products of formaldehyde, *glutol, *amyloform, etc., are more expensive and less constant in their action than tannoform. Liquor formaldehydi in 1 to 2 per cent. solution is a strong disinfectant but very irritant to wounds; in concentration it is a strong caustic (caution!).

Tinctura iodi. Tincture of iodine has been employed recently to disinfect the skin in place of the older and more complicated process (method of Grossich), being simply painted on the field of operation.

Argenti nitras. Nitrate of silver is an excellent wound remedy. 
It possesses a high disinfection value (a 1 to 1000 solution destroys pus cacci), is an important regulator of abnormal granulations and covers the wound with a scab (silver scab). This is also true of the other very expensive silver preparations (*actol, *itrol, *protargol).

*Aluminum acetas. Acetate of aluminum. An excellent, nonpoisonous antiseptic in 2-8 per cent. aqueous solution (also contained in Burow's mixture ${ }^{2}$ ); preferred on account of its cheapness to the more expensive substitutes *alumnol, *tannal, *gallal, *sozal, *boral, *saluminal, *kutol, etc.

Alcohol. An important disinfectant for the hands of the operator and for the skin of the operation field; acts best in $\mathbf{5 0}$ per cent. solution in water or sublimate solution (absolute alcohol has only a weak antiseptic action). Soap spirit ${ }^{3}$ is also recommended. The alcoholic tincture of aloes [tinctura aloes] is an excellent antiseptic and stimulant to granulation for old wounds.

Acidum salicylicum. Salicyclic acid is a very mild antiseptic, but is non-toxic, odorless and non-irritant; used especially in ophthalmology, also to wash out internal organs. Thioform (dithiosalicylate of bismuth) is recommended as a substitute for iodoform, especially because it is odorless and non-toxic (but is very expensive).

Bismuth salts. Like tannoform, these are employed as desiccant, astringent, dry antiseptics, but are very expensive. Those most frequently used are bismuthi subnitras, subsalicylas, subgallas (dermatol), and dithiosalicylas (thioform), and *airol (iodized dermatol). The latter in the form of airol paste ${ }^{4}$ is an excellent substitute for dressings and bandages for wounds; it is aseptic, non-irritant, easily applied, adhesive, plastic and dries quickly.

Acidum boricum. Boric acid is a mild, non-toxic, odorless antiseptic without pronounced action (ophthalmology). Sodii boras, *magnesii boras, *boral, *antipyonin, *rotterin, *antiseptin, *borol and other preparations containing boric acid act in the same manner.

[2 Alum 5 parts, lead acetate 10 parts, water 250 parts.]

[3 Equal parts of green soap and alcohol.]

["Airol, glycerin and acacia, each 1 part, argilla 2 parts.] 
Zinci chloridum. Chloride of zinc is a caustic, alterative antiseptic ( 2 to 8 per cent. solution) of relatively weak disinfection power (a regulator of abnormal granulations).

Potassii permanganas. Permanganate of potassium is a mild antiseptic; specific against snake bites and rabies virus.

Camphora. Camphor is a powerful antiseptic, especially for "flabby" granulations and phlegmonous, ulcerative and necrotic processes (camphor spirit bandage). Oleum terebinthinæ, terebinthina (old hoof remedy), thymol, oleum eucalypti, balsamum peruvianum and other ethereal oils act in the same manner.

Pix liquida. Tar. An excellent antiseptic, especially for wounds on the hoof and claws; wood tar is better than coal tar.

Liquor chlori compositus. Chlorine water. A strong antiseptic; specific against rabies virus and snake bites; used in ophthalmology.

*Pyoctanin. A powerful antiseptic. Disadvantage: blue stain.

\section{INTERNAL ANTISEPTICS}

A number of infectious diseases may be effectively combated by the internal use of antiseptics. The antiseptics used for this purpose include some of the specifics: salicylic acid, the principal remedy in acute articular rheumatism; quinine, the specific for malaria; mercury, salvarsan and iodine as specifics for syphilis of man, salvarsan and atoxyl as specifics against trypanosomes and spirilla, and salvarsan in contagious pneumonia of horses ("Therapia sterilisans magna"); also creolin, which is very effectiveagainst anthrax in cattle and horses; creosote, as an internal remedy in tuberculosis; calomel, salicyclic acid, bismuth salts, tannin and boric acid as intestinal disinfectants in dysentery, influenza, canine distemper, swine erysipelas, fowl cholera and mycotic and infectious inflammations of the intestines; and tar and oil of turpentine in infectious and parasitic affections (lung worm disease) of the respiratory tract. Infectious materials in the blood are also destroyed through serum therapy (see the chapter on protective vaccination and methods of immunization). In internal antisepsis, even more than with external antisepsis, care must be taken to avoid a general poisoning of the body. 


\section{THE CONSERVATION OF ANIMAL PRODUCTS}

Methods of Conservation. - The conservation of organic animal substances has for its purpose the prevention of the decomposition, putrefaction and fermentation processes which occur, as a rule, after the death of the animal organs. This is accomplished by the destruction of the microörganisms concerned in the decomposition and fermentation processes and by preventing the access and development of additional organisms. In most cases the conservation concerns meat and milk; in addition, cadavers, hides and other parts of the carcass also require attention. Since the microörganisms concerned can only multiply in an atmosphere with a certain oxygen content and a certain amount of moisture, within certain temperature limits, and upon proper nutritive media, they may be destroyed in different ways in addition to the employment of antiseptics.

1. The conserving agents which act antiseptically correspond essentially with the disinfectants. Usually, several substances are used together to increase the conserving action. A distinction must be made between the non-toxic agents, which can be used to conserve meat and milk, and the toxic substances, which serve to preserve parts of cadavers. The non-toxic meat and milk preservatives are: sodium chloride, potassium nitrate (salting, pickling), sodium phosphate, sodium acetate, vinegar, sugar, glycerin, smoking and hydrogen peroxide. The mixtures of conserving agents must not contain toxic substances or those which are not entirely non-toxic (boric acid, salicylic acid, sulphurous acid, fluoride of soda, ammonium acetate, formalin, potassium permanganate). Poisonous preservatives for parts of cadavers are corrosive sublimate, arsenic, hydrocyanic acid, creosote, carbolic acid, methyl alcohol, chloroform, ether, acetone, chloral, copper sulphate, zine sulphate, iron sulphate, zinc chloride, copper chloride, aluminum chloride, iron chloride, alum and hyposulphites. Wickersheimer's conserving fluid for cadavers (arsenic 10 grams, sodium chloride 25 grams, potassium nitrate 12 grams, potash 60 grams, methyl alcohol 1 liter, glycerin 4 liters, water 10 liters) is well known. 
2. Exclusion of air. This very simple method of conservation is usually used in connection with previous heating (sterilization, pasteurization). The simple exclusion of air is obtained by the different paints (lacquer, oil paints, tar, sealing wax, resin) and by the application of a layer of fat, oil or sugar (sardines, gooseliver pie, sweet conserves). Cotton and asbestos stoppers prevent the entrance of the bacteria contained in the air; this method is used principally in bacteriological technique. Finally, the air can be expelled by carbon dioxide (canned meat).

3. High and low temperatures have a conserving action when they are considerably above or below 4 to $45^{\circ} \mathrm{C}$., the optimum temperatures necessary for the development of microörganisms. Sterilization is the process of heating to $100^{\circ} \mathrm{C}$. or over. Pasteurization is the process of heating to 65 to $100^{\circ} \mathrm{C}$. Temperatures of high degree in the form of boiling-heat (frequently with simultaneous or subsequent exclusion of the air), hot air and live steam are essentially more effective than lower temperatures; they are especially used in the preparation of canned meats (corned beef). Lower temperatures in the form of freezing or storage in coolingrooms, ice cellars and refrigerators are less effective because the spores are not destroyed but only hindered in their development and also because some of the bacteria are only temporarily benumbed. Substances conserved in this manner therefore undergo decomposition very rapidly when they are removed from the influence of the cold, since the microörganisms contained in them immediately begin to multiply. This has been the experience with the cold-storage rooms in slaughter houses and with the oversea transportation of meat.

4. Withdrawal of moisture is attained by drying, either through the use of the sun's heat (codfish) or through artificial heat (carna pura, meat meal). In the preparation of meat meal, the meat, after the removal of the fat and bones, is finely chopped and dried in an oven at $60^{\circ} \mathrm{C}$., after which it is finely powdered and enclosed in cans. The so-called condensed milk is also prepared by withdrawing water (evaporated to one-fourth the volume), and sugar is added to better preserve it. 


\section{ANTIDOTES}

Synonyms: Antitoxics, antagonistics, antagonists.

Classification.-Medicines which are employed in different poisonings are called antidotes. They are of different kinds and operate in different ways. The following groups may be distinguished: (1) The mechanical or physical antidotes; (2) the chemical antidotes; (3) the physiological (dynamic, organic, constitutional and empirical) antidotes or antagonists, and (4) the symptomatic method of treating poisoning.

1. The physical or mechanical antidotes operate in a purely mechanical way by removing the poison from the body: emetics, cathartics, diuretics, diaphoretics, sialagogues, washing out the stomach (horse, dog), bleeding, transfusion, artificial respiration; or by enveloping the poison and preventing its absorption or contact with the mucous membrane: protective antidotes (albumin, milk, oil, mucilaginous substances).

Of special practical importance are the emetics, which are immediately administered to the proper animals (dogs, swine and cats) in all fresh cases of poisoning. The most important emetics are apomorphine (dogs, $0.002-0.01$, gr. $1 / 40$ to $1 / 8$; cats, $0.02-0.05$, gr. $1 / 4$ to $3 / 4$, subcutaneously), veratrin (swine, $0.02-0.03$, gr. $1 / 4$ to $1 / 3$, subcutaneously), veratrum alba (swine, $1-2$, grs. $\mathrm{xv}$ to $\mathrm{xxx}$; dogs, $0.1-0.2$, gr. $1 / 8$ to $1 / 4$, per os or in clyster), ipecac (swine and dogs, 1-3, grs. xv to xlv; cats, $0.25-0.75$, grs. iij to xj), tartar emetic (swine, 1-2, grs. $\mathrm{xv}$ to $\mathrm{xxx}$; dogs, 0.1-0.3, grs. iss to iv; cats, $0.05-0.2$, grs. $3 / 4$ to iij), copper sulphate in phosphorus poisoning (swine, $0.5-1$, grs. vij to $\mathrm{xv}$; dogs, $0.1-0.5$, grs. iss to vij; cats, $0.05-0.2$, grs. $3 / 4$ to iij), zinc sulphate (swine, $0.5-1$, grs. vij to $\mathrm{xv}$; dogs, $0.1-0.3$, grs. iss to iv), and finally the household emetics, sodium chloride (dogs, 1 to 2 teaspoonfuls), mustard (dogs, 1 to 2 teaspoonfuls in a glass of warm water), snuff (dogs, a pinch in a tablespoonful of water), etc. Of the cathartics, eserine (0.050.1 , grs. $3 / 4$ to iss, for horses) and arecoline (0.05-0.08, gr. $3 / 4$ to 1 , for horses) are especially suitable on account of their rapid action; others are aloes (horses, $25-50,3 \mathrm{vj}$ to xii; cattle, 50-75, 5 iss to 216 
ijss); castor oil (horses, 250-500, Oss to i; cattle, 500-1000, Oi to $\mathrm{ij}$; sheep and goats, 50-250, 予 iss to viij; swine, 50-100, 节 iss to iij; dogs, $15-60$, 5 ss to $\mathrm{jj}$; cats and fowl, 10-30, 3 ijss to $5 \mathrm{j}$ ), which, however, is to be avoided in phosphorus, arsenic and cantharides poisoning (solution of the poison); calomel (swine, 1-4, grs. $x v$ to $3 \mathrm{i}$; dogs, 2-4, grs. xxx to $3 \mathrm{i}$; cats and chickens, 0.1 , grs. iss); Glauber's and Epsom salts (in lead poisoning, also a chemical antidote). The diuretics, diaphoretics and sialagogues have a much weaker evacuating action and are therefore employed only in chronic poisonings. The protective antidotes are used principally in poisonings by caustics to protect the gastric mucous membrane and to prevent absorption. Those used most frequently are milk, albumin (egg albumin alone or shaken up with water), mucilages (linseed, barley, oat and quince mucilage, acacia, decoctions of althæa root, mallow leaves and salep, tragacanth mucilage), fats and oils (lard, butter, peanut oil, olive oil, rape oil, poppy-seed oil, almond oil, castor oil, emulsions). The fatty oils, however, are contraindicated in phosphorus and cantharides poisoning because they promote the resorption of these substances; this is also true of milk or any compound which contains fat.

2. The chemical antidotes prevent poisoning by decomposing the poison or by changing it into a compound which is non-toxic or less poisonous. The simplest example of a chemical antidote is presented by the caustic alkalies and acids, which in combining lose their opposing alkaline and acid characteristics and form neutral salts which are not caustic (potash lye, soda lye, caustic lime, ammonia, carbonate and bicarbonate of soda and of potassium, and soap on the one hand, and sulphuric, hydrochloric, nitric, acetic and oxalic acids on the other hand). This group also includes sodium chloride, the specific antidote for silver nitrate, which it transforms into silver chloride (the administration of sodium chloride in corrosive sublimate poisoning is harmful because it promotes the resorption of the sublimate); iron, iron hydroxide and iron oxide, the antidotes for arsenic (formation of arsenate of iron, which is only slightly soluble), hydrocyanic acid, mercury and copper salts; potassium ferrocyanide, the antidote 
for copper poisoning (non-poisonous ferrocyanide of copper) and for caustic iron salts, e.g., iron chloride (formation of ferrocyanide of iron); copper salts, the important antidote for phosphorus poisoning (formation of insoluble phosphorus copper), magnesia oxide and magnesia carbonate, the antidotes for acids (formation of salts of magnesia), arsenic and metallic salts (decomposition); iodine and the iodides, the antidotes for the alkaloids in general (precipitation) and for chronic metallic poisonings (formation of metallic iodides, which are more soluble and therefore more readily eliminated from the body); potassium bromide, which combines with iodine and iodoform, forming potassium iodide; sulphur and potassium sulphide (hydrogen sulphide), antidotes for mercury, lead, copper, tartar emetic and arsenical poisonings (formation of insoluble metallic sulphides); sulphuric acid and its salts, specific antidotes for lead poisoning (formation of insoluble lead sulphate), carbolic acid poisoning (formation of non-poisonous potassium sulphocarbolate), calcium poisoning (formation of plaster-of-Paris) and barium poisoning (formation of barium sulphate); the calcium salts (lime water, calcium carbonate, chalk, snail shells, oyster shells, egg shells, marble, sepia stones, sugar lime), antidotes for oxalic acid poisoning (calcium oxalate is insoluble and consequently not poisonous); tannic acid and the plants which contain it (oak bark, cortex salicis, salvia leaves, coffee, tea, acorns, nutgall, ink, cinchona, catechu, rhatany root, tormentilla, walnut tree leaves), important antidotes for poisoning by alkaloids and glucosides (formation of slightly soluble or insoluble tannates), also for poisoning by the metallic salts, especially tartar emetic, lead acetate, silver chloride and iron sulphate (formation of metallic tannates); ammonia, chlorine and potassium permanganate, local antidotes for snake bites and insect stings; old oil of turpentine and ozone water, antidotes for phosphorus (oxidation to phosphoric acid), albumin, antidote for the metallic salts (formation of metallic albuminates), caustic acids (formation of serum albumins) and chlorine, bromine and iodine poisoning; gelatin and gum, antidotes for metallic poisonings (sublimate poisoning), alum and tannic acid poisoning (formation of 
precipitates); fatty oils, antidotes for poisoning by alkalies and acids (formation of soap); starch, antidote against iodine (blue color), and animal charcoal, antidote for alkaloids and metals.

3. The physiological or dynamic antidotes are not directed against the poison itself but against its action (antagonists), and are administered for the purpose of producing an action which will counteract the effect of the poison (stimulation-paralysis). A distinction must be made between a single, or simple, and a multiple, or mutual antagonism. The antagonism is single when the one poison only suppresses but does not reverse the action of the other; it is multiple when a reciprocal or mutual suppression takes place. We also speak of a true (direct) and an apparent antagonism, depending upon whether both poisons act upon one and the same organ (nervous system, muscles, glands) or upon different organs; curare, for instance, is only an indirect antagonist of strychnine because it does not act upon the spinal cord, as does strychnine, but upon the peripheral nerves of the muscles. The occurrence of a true multiple antagonism is ingeneral questionable. A paralyzing antidote like atropine can, it is true, overcome the stimulant action of another poison, e.g., the action of eserine upon the oculomotor nerve (mydriasis, myosis), but on the other hand a paralysis of the oculomotor nerve (mydriasis) produced by atropine cannot be overcome by eserine (myotic). The important physiological antidotes are: (a) Atropine and hyoscine (scopolamine) as antidotes against morphine, chloroform and chloral hydrate. The antagonism is multiple but in part indirect. The paralytic action of morphine on the brain is overcome directly by the psychic stimulation produced by the atropine; on the other hand, the paralytic effect of the morphine on the heart is overcome by the stimulant action of the atropine indirectly, the atropine stimulating the nerve centres of the heart, while morphine paralyzes the heart muscle itself. (b) Atropine as an antidote against pilocarpine, eserine, arecoline, muscarine and nicotine poisoning. (c) Potassium bromide, chloral hydrate, chloroform and ether as antidotes against the tetanics, strychnine and picrotoxin. (d) Amyl nitrite as an antagonist against the vasoconstrictors, suprarenin 
[adrenalin] and ergot. Caffeine as an antidote against morphine, chloroform and alcohol.

4. The symptomatic antidotes are used to treat individual symptoms of poisonings. Symptoms of paralysis are combated by excitants (camphor, ether, alcohol, wine, caffeine, coffee, tea, veratrin, strychnine, atropine, scopolamine, ammonia, carbonate of ammonia); painful colics by morphine and other narcotics; diarrhœa by styptics; constipation by laxatives; persistent vomiting by opiates; high fever by antipyretics; cramps by sedatives, and subnormal temperatures by caffeine.

Antidotes for the Different Poisons.-Aconite. Emetics, tannic acid, iodine, animal charcoal, atropine, digitalis, artificial respiration, excitants.

Alcohol. Coffee, caffeine, ammonia, ammonium carbonate, camphor, atropine, scopolamine, cold applications to the head, warm coverings.

Alkalies, caustic. Vinegar, dilute acids (hydrochloric, sulphuric, phosphoric, tartaric and citric acids), fatty oils, milk, emulsions, mucilaginous substances, morphine.

Aloes. Opium, tannic acid, mucilaginous substances.

Alum. Albumin, milk, gelatin solution, lime water, magnesium oxide, ammonia, laxatives.

Ammonia. Dilute acids, vinegar vapor, fats, oils, milk, mucilaginous substances, tracheotomy.

Anilin. Emetics, laxatives, excitants.

Arecoline. Atropine, hyoscine (scopolamine).

Arsenic. Emetics, iron preparations, iron hydrate [ferri hydroxidum cum magnesii oxido, U. S. P.], *ferric saccharate, magnesium oxide, animal charcoal, sulphur, liver of sulphur, sulphide of iron, albumin, milk, mucilage, excitants. Alkalies and fatty oils (castor oil) are to be avoided.

Atropine (belladonna, henbane, stramonium). Emetics, tannic acid, morphine, pilocarpine, eserine; for the psychic excitement, chloroform, chloral hydrate, sulphonal, potassium bromide.

Barium. Dilute sulphuric acid, sodium and potassium sulphate, magnesium sulphate, emetics, atropine. 
Brine. Plenty of water, mucilage, oil, excitants, sedatives.

Cantharides. Mucilaginous substances, opium, excitants; no fatty oils.

Carbolic acid. Emetics, wash out the stomach, sulphates, dilute sulphuric acid, soap water, sugar lime, albumin, milk, oil, excitants (ether, camphor, caffeine, hyoscine).

Carbon disulphide. Fresh air, excitants.

Carbon monoxide. Fresh air, inhalation of oxygen, infusion of physiological salt solution; excitants, artificial respiration, stimulation of the skin.

Chick-pea. Tracheotomy, strychnine, veratrin, caffeine, atropine, cutaneous stimulation, laxatives.

Chlorine and calcium chloride. Sodium or magnesium subsulphate, inhalations of hydrogen sulphide and ammonia (diluted); internally, aromatic spirit of ammonia; albumin, mucilage.

Chloroform, chloral hydrate, or ether. Excitants, especially atropine, hyoscine (scopolamine), strychnine, veratrin, caffeine, ammonia, ammonium carbonate, cutaneous stimulation, cold douches, artificial respiration.

Chromic acid. Albumin, magnesium oxide, excitants.

Cocaine. Tannic acid, iodine, chloral hydrate.

Colchicum. Emetics, tannic acid, iodine (Lugol's solution), opium, morphine, mucilage, warm moist coverings, excitants.

Conium. Emetics, laxatives, veratrin, strychnine, caffeine, atropine, ether, camphor, ammonium carbonate, tannic acid.

Convallaria. Tannic acid, excitants, camphor, ether.

Copper. Laxatives, emetics, albumin, potassium ferrocyanide, powdered iron, magnesium oxide, animal charcoal, excitants.

Corn cockle. Laxatives, tannic acid, mucilage, excitants.

Creosote. Emetics, mucilage, dilute sulphuric acid, sodium sulphate, magnesium sulphate, soap, excitants.

Croton oil. Mucilage, albumin, opium, excitants.

Cystisus. Emetics, laxatives, excitants.

Darnel. Laxatives, ether, camphor, atropine, caffeine, cutaneous stimulation.

Digitalis. Emetics, tannic acid, camphor, ether, atropine, 
scopolamine, caffeine, aromatic spirit of ammonia, wine, cutaneous stimulation.

Equisetum. Laxatives, camphor, ether, caffeine, atropine, hyoscine (scopolamine), veratrin, cutaneous stimulation.

Ergot. Emetics, laxatives, chloral hydrate, amyl nitrite, tannic acid, symptomatic treatment.

[Ether. See Chloroform.]

Fungi. Emetics, laxatives, excitan is, symptomatic treatment.

Glauber's salt. Mucilage, opium, camphor, ether.

Hellebore. Tannic acid, opium, excitants.

Hydrochloric acid. Dilute alkalies, albumin, mucilage, oil, opium.

Hydrocyanic acid. Emetics, hydrated oxide of iron, hydrogen peroxide, potassium permanganate, cobalt nitrate, atropine, artificial respiration, excitants, cold douches.

Hydrogen sulphide. Fresh air, ether, camphor, inhalation of chlorine, excitants, cutaneous stimulation, bleeding, physiological salt solution subcutaneously.

Illuminating gas. Fresh air, inhalation of oxygen, cutaneous stimulation, artificial respiration.

Insect stings. Wash with ammonia water, chlorine water, alcohol, lead water.

Iodine and iodoform. Starch, flour water, albumin, potassium bromide, sodium bromide, sodium and potassium bicarbonate, sodium hyposulphite, excitants, emetics, laxatives.

Lead. Emetics, laxatives, dilute sulphuric acid, sodium sulphate, potassium sulphate, magnesium sulphate, sulphur, hydrogen sulphide, tannic acid, albumin, milk, symptomatic treatment. (opium, morphine). In chronic lead poisoning, potassium iodide.

Lupine. Change of feed, preparation of the poisonous lupines, dilute acids (no alkalies!), castor oil.

Male fern. Laxatives, excitants.

Mercurialis. Emetics, albumin, milk, opium, tannic acid.

Mercury. Albumin, milk, powdered iron, sulphur, sulphurated potash, hydrogen sulphide, magnesium oxide, symptomatic treatment; in chronic poisoning, potassium iodide. 
Mine gas. Fresh air, artificial respiration, cutaneous stimulation, cold douches, excitants.

Morphine. Atropine, hyoscine (scopolamine), caffeine, coffee, tea, cutaneous stimulation.

Muscarine. Atropine, hyoscine.

Mushrooms. Laxatives, demulcents, excitants, cutaneous stimulation, tannic acid, iodine (atropine in poisoning by agaricus muscarius).

Nicotine. Laxatives, tannic acid, iodine solution, animal charcoal, excitants, rumenotomy.

Nitric acid. Dilute alkalies, albumin, mucilage, oil, opium.

Oleander. Laxatives, mucilage, tannin, excitants.

Opium. Atropine, hyoscine, caffeine, laxatives, excitants.

Oxalic acid. Lime water, sugar lime, chalk, magnesium oxide, excitants, diuretics.

Petroleum. Emetics, cutaneous stimulants, ether, camphor, wine, ammonium carbonate, caffeine, artificial respiration.

Phosphorus. Emetics, laxatives, old oil of turpentine, copper sulphate, copper salts in general, potassium permanganate, cobalt nitrate, hydrogen peroxide, excitants, infusion of physiological salt solution; fats and fatty oils are to be avoided.

Physostigmine. Atropine, hyoscine (scopolamine), symptomatic treatment.

Pilocarpine. Atropine, hyoscine, agaricin, excitants.

Poppy (wild). Laxatives, tannic acid, opium, morphine, chloral hydrate, potassium bromide, cold shower bath upon the head, clysters.

Potassium chlorate. Emetics, infusion of physiological salt solution, excitants.

Potassium nitrate. Mucilaginous substances, oil, ether, alcohol, wine, camphor, ammonium carbonate, cutaneous stimulation.

Ptomaines. Emetics, laxatives, tannic acid, iodine water, animal charcoal, calomel, ether, camphor, caffeine, atropine, wine, ammonia, symptomatic treatment.

Ranunculus. Emetics, laxatives, tannic acid, excitants.

Sabina. Mucilaginous, demulcent remedies, opium, morphine. 
Santonin. Emetics, laxatives, ether, chloral hydrate, camphor, wine, symptomatic treatment.

Silver Nitrate. Emetics, albumin, sodium chloride, dilute hydrochloric acid.

Snake venom. Locally, chlorine water, calcium chloride solution, iron chloride, solutions of potassium permanganate, ammonia, carbolic acid or creolin; internally, alcohol in large doses, ether, camphor, atropine, caffeine, hyoscine (scopolamine), aromatic spirit of ammonia.

Sodium chloride. Plenty of water, mucilage, oil, ether, camphor, atropine, caffeine, antispasmodics when indicated.

[Sodium sulphate. Mucilage, opium, camphor, ether.]

Solanine. Tannic acid, laxatives, excitants.

Strychnine. Chloral hydrate, chloroform, potassium bromide, ether, sulphonal, morphine, artificial respiration, tannic acid, iodine water, emetics, irrigation of the stomach.

Sulphuric acid. Dilute alkalies, lime water, soda, magnesium oxide, chalk, mucilage, oil, excitants.

Tannic acid. Albumin, gelatin, mucilage, laxatives.

Tartar emetic. Tannic acid, sodium carbonate, dilute acids, sulphur, sulphurated potash, opium, albumin, mucilage, excitants.

Tobacco. See Nicotine.

Turpentine oil. Mucilaginous substances, opium, excitants.

Veratrin. Tannic acid, iodine, opium, sedatives, demulcents, excitants.

[Veratrum. See Hellebore.]

Verdigris. Albumin, potassium ferrocyanide, powdered iron, magnesium oxide.

Vinegar. Soap water, soda water, chalk, calcium carbonate, magnesium oxide, milk, excitants.

Yew tree. Laxatives, excitants, atropine, hyoscine, caffeine, veratrin, strychnine, camphor, ether, alcohol, ammonia, iodine (Lugol's solution).

Zinc. Albumin, mucilage, milk, tannic acid, opium, sodium and potassium carbonate and bicarbonate, sulphurated potassium, excitants. 


\section{VACCINATION. IMMUNIZATION. INOCULATION}

\section{IMMUNITY, MITIGATION, AND METHODS OF INOCULATION}

Immunity. - It was early observed that after recovery from certain infectious diseases the animal or human body was protected against a new infection. This protection against infection is called immunity and the animal endowed with this property of insusceptibility is said to be immune or refractory to a specific infection. There are different varieties of immunity. Species immunity is the natural insusceptibility of an entire animal species to certain infectious diseases. For example, cattle are immune to glanders, the horse to lung plague and rinderpest, the dog and the cat to swine erysipelas, man to fowl cholera, the rabbit to black-leg, and almost all species of animals to syphilis, scarlet fever, and measles. The immunity may even be limited to certain breeds of a species. The Algerian sheep, unlike the other breeds, are alleged to be immune to anthrax. The German native swine and the Yorkshire swine are much less susceptible to erysipelas than the other breeds; compared with field mice, house mice and white mice are immune to tuberculosis and glanders. This variety of immunity is due to certain unknown properties peculiar to the species and breeds, Individual immunity refers to the insusceptibility of single individuals of the same animal species to this or that infection to which the members of the species are susceptible. It is frequently observed in an outbreak of foot-and-mouth disease in cattle, or of contagious pneumonia, influenza and strangles of horses, that a large percentage of the animals in a stable remain free from infection.

Both of the aforementioned varieties of immunity, species and individual immunity, fall under the general term of natural 
immunity, which is also called inherited immunity. It is due to an inherited special property of the body cells and the blood which cannot be transmitted to other animal species. An acquired immunity, on the other hand, is established after an animal has passed through a single attack of a certain disease and lasts for a certain time (immunity after recovery). This variety of immunity may follow an attack of pox, anthrax, lung plague, foot-and-mouth disease, strangles, canine distemper, black leg, swine erysipelas, etc., and also scarlet fever, measles, typhoid fever and diphtheria of man.

The acquired immunity is to be attributed to the presence of specific substances in the body (blood, blood-serum) which reduce the susceptibility of the organism to the infectious agents which caused the disease. These immunizing substances either neutralize the toxins formed by the invading bacteria (antitoxic action, toxic immunity) or they destroy the pathogenic bacteria themselves (bactericidal action, bacterial immunity). In contrast to natural immunity, acquired immunity may be transmitted from one animal species to another (pox from cattle to man).

Finally, two varieties of acquired immunity are recognized, the active and the passive. The active immunity is acquired spontaneously by an animal in passing through an attack of the particular disease (natural or artificial infection). This immunity can be increased by a repetition of the infection and can usually be made more continuous. It is due to the formation in the bloodserum of antibodies (antitoxins, bacteriolysins, bactericides). The passive immunity is produced by the artificial introduction of the antitoxins (secondary immunity). It is only of short duration because the antibodies artificially introduced are soon used and none are formed to replace them, as occurs in active immunity (inoculation of blood-serum in contagious pneumonia of horses, foot-and-mouth disease, rinderpest, and swine erysipelas). In vaccination against swine erysipelas the swine are passively (serum inoculation) as well as actively (inoculation of bacilli) immunized. 
Practical experience having taught that an acquired immunity often existed, attempts were early made, by intentionally introducing infection, to produce an artificial protection against a subsequent infection. This variety of insusceptibility is called artificial immunity and the process of artificial infection is termed vaccination. The first vaccination for the purpose of producing an artificial immunity was Jenner's vaccination of man with cow pox (1796). 1 In veterinary medicine, vaccinations of this character were early undertaken (sheep pox, lung plague, rinderpest, foot-and-mouth disease). In more recent times, great scientific and practical interest has been manifested in the production by vaccination of artificial immunity against anthrax and black leg, rabies, swine erysipelas, swine plague, tuberculosis, calf cholera, chicken cholera, contagious pneumonia of horses, and tetanus. Vaccination has become a valuable method of combating the infectious diseases and is a species of internal disinfectant and antidotal treatment.

Causes of Immunity.-The explanation of the establishment of immunity has always been one of the most difficult therapeutical problems. Only in recent times has light been thrown on the intricate question by histological, bacteriological, and chemical inquiries. But a conclusive investigation has not been made, nor has an entirely satisfactory explanation of the causes and nature of immunity been discovered.

The best-known theories at this time are the serum theory of von Behring and the phagocytic theory of Metschnikoff. The first has his supporters chiefly in Germany, the latter in France. The truth may indeed lie between the two; namely, that the serum and the phagocytes both act as protectives of the body against infectious materials.

1 Jenner's celebrated paper, which appeared in London in 1798, bore the title: "An Inquiry into the Causes and Effects of the Variolæ Vaccinæ, a Disease Discovered in Some of the Western Counties of England, Particularly Gloucestershire, and Known by the Name of Cowpox." Jenner's discovery has become the greatest therapeutic fact of all times. 
1. The serum theory of von Behring locates the immunizing substances in the cell-free blood-serum. Under the influence of the pathogenic microörganisms the body cells produce chemical substances with disinfectant properties (antibodies, alexins, immune proteins) which destroy the bacteria (bactericidal action) either by dissolving (bacteriolysins), clumping (agglutinins), or precipitating (precipitins) them, or which neutralize their toxins (antitoxins) or facilitate phagocytosis (opsonins). Artificially inoculating the antitoxins in the form of the blood-serum of immune animals into healthy animals produces in the latter passive immunity; if the material is injected into diseased animals recovery occurs (von Behring's law; serum therapy). These antitoxins have not up to this time been isolated in their pure state. According to von Behring, this is not possible principally because they are not an antitoxin material but an antitoxic force similar to the magnetic force of iron (!). Von Behring has differentiated in vaccination the isotherapeutic (inoculation of the bacteria themselves, Jennerization, cow pox vaccination, vaccination against tuberculosis of cattle) and the homcotherapeutic principle (inoculation of the specific serum in diphtheria, swine erysipelas, tetanus) (see p. 22).

Modifications of the serum theory have been formulated by Buchner, Ehrlich, Brieger, Emmerlich, Wassermann, Lorenz, and others. According to Buchner, the antitoxic body is an enzyme with a ferment-like action

2. The phagocytic theory of Metschnikoff is, on the contrary, a cellular theory, which places the immunizing force in the white blood-cells (leucocytes, phagocytes). In infectious diseases, these cells remove the invading infectious agents and their pathogenic products from the blood by taking them within the cell body (leucocytosis, phagocytosis). In local infection (inflammation), they leave the blood-vessels in the form of wandering cells or pus cells in order to take up the infectious material (positive chemotropism or positive chemotaxis). In addition to this higher susceptibility of the cells, there also occurs through adaptation an 
indifference of the cells toward the infectious material, in consequence of which the cells avoid the pathogenic bacteria (negative chemotropism or chemotaxis). "Living cells, governed by their susceptibility, approach pathogenic microbes or flee from them; take them within the cell body or allow them to lie free." The Metschnikoff theory received considerable support from the discovery by Pfeffer of the law of the chemotropism of vegetable cells (attraction or repulsion by certain chemical substances). The behavior of the white blood-cells in inflammation, which is analogous to the chemotaxis of plant cells, was first demonstrated by Leber.

Other Theories.-1. The Exhaustion Theory (Pasteur). The first attempt to explain the cause of immunity was made by Pasteur. In his studies of fermentation he observed that the ferment organisms became ineffective after a certain time, when they had used up a certain quantity of the nutritive material from the nutrient solution, and he concluded that the pathogenic organisms likewise withdraw from the invaded body certain substances indispensable to their development, so that the body is in a certain sense exhausted and microörganisms invading the body later fail to find suitable nutritive material. Similarly, the so-called soil exhaustion is observed after longcontinued planting of the same fodder crop.

2. The Aggressin Theory (Bail). Aggressins is the term used by Bail to describe the bacterial products which are formed, especially at the point of infection, to assist the bacteria in the combat between them and the leucocytes. The aggressins possess negative chemotactic properties, as a result of which the leucocytes are repelled. They are found especially in the cedema at the point of infection (in anthrax) and in peritonitic and pleuritic exudates. The injection of such "aggressive exudates" produces active immunity. The blood-serum of an actively immunized animal contains passively immunizing "anti-aggressins."

3. The Opsonic Theory (Wright). According to Wright, the specific action of serum is due to opsonins, in addition to antitoxic and bactericidal substances. Opsonins are protective substances which promote phagocytosis by making the bacteria more readily digestible by the leucocytes (bacteriotropic substances).

4. The Assimilation Theory (Baumgarten). The blood of immune animals contains no antitoxins (no one has demonstrated them!), but is so changed chemically that the pathogenic organisms can no longer develop in it. (See the colyseptic action of the disinfectants.) 
5. According to Much (Immunitätswissenschaft, 1911), a distinction must be made between toxin bacteria and endotoxin bacteria in considering the immunizing processes occurring in the different infectious diseases.

I. The toxin bacteria remain at the point of infection (tetanus, diphtheria, sausage poisoning) and act pathogenically by the excretion of poisons (bacterial poisons or toxins). The antitoxins are the antidotes, which appear in the blood-serum in consequence of the presence of the toxins. The antitoxic serum acts against the toxins as well as against the bacteria.

II. The endotoxin bacteria spread through the body (septicæmia, streptococci) and exert a pathogenic effect not only through the toxins excreted but also through the cell substances of the bacteria (endotoxins) when they disintegrate in the animal body. They are not counteracted by antitoxins, but the substances which protect the body against these organisms are: 1, normal humoral bacteriocidins, i.e., the bactericidal substances which are present in solution in every normal serum (serum substances); 2 , normal leucocytic bacteriocidins, i.e., the bactericidal substances in solution which originate from the leucocytes (plasma substances); 3 , specific humoral bacteriocidins, which appear in the serum in the later stages of the infection-these three protective substances destroy the living organisms; 4 , the poisonous substances of the endobacteria, the endotoxins, are destroyed through phagocytosis by the leucocytes, while phagocytosis is again stimulated by the bacteriocidins.

The Weakening (Mitigation) or Attenuation of the Infectious Materials. - In every protective or curative vaccination it is important that the infectious material be used in a weakened, mild (mitigated), diluted (attenuated) form, since the unweakened, unattenuated virus will produce a severe or fatal infection. The methods and manner of attenuation are very different. The most important, arranged in historical order, are the following:

1. The introduction of a small quantity of the infectious material. The greater the number of a pathogenic organism in the body the more pronounced the effect; therefore, in vaccination care must be exercised that only a minimum quantity of the infectious material is inoculated, otherwise the original disease will be produced in undiminished strength.

2. The selection of a different entrance point for the infectious material. Under ordinary conditions the contagion of most infections is taken in through the internal organs, especially the respiratory and digestive apparatus. This has been demonstrated 
in connection with lung plague, foot-and-mouth disease, rinderpest, pox, swine erysipelas, chicken cholera and other diseases. If the contagion of these diseases is introduced into the body through another channel, for example through the skin or subcutis, it will be brought in contact with essentially different living conditions and it will also be absorbed very slowly and in very small quantity. This method of attenuation was formerly employed exclusively in veterinary medicine and possesses for the present and for the future of vaccination a continuing importance. At the present time, for instance, the contagion of pox and of foot-and-mouth disease is inoculated in the skin or mucous membrane (cutaneous), and that of lung plague, black leg, swine erysipelas, rinderpest, etc., is injected subcutaneously for the purpose of diminishing the course of the disease. Furthermore, a place for the inoculation is selected as far removed from the heart as possible in order that the resorption will be very slow (end of the tail, tip of the wing, ear).

3. The influence of the higher degrees of temperature. Every organism has its optimum and its maximum temperature. Higher degrees of temperature weaken the vital energy and finally destroy the organism. It is therefore possible to diminish the toxic effect of the different infectious materials by the use of a certain degree of heat. The reduction of the virulence by heating for 10 to 15 minutes at 50 to $55^{\circ} \mathrm{C}$. was first demonstrated by Toussaint in connection with the anthrax bacillus. The heating process is of great practical importance in Pasteur's protective anthrax vaccination, in which one part of the anthrax bacilli is exposed for 24 days to a constant temperature of $42^{\circ}$ to $43^{\circ} \mathrm{C}$. (first vaccine) and the other part to the same temperature for only 12 days (second vaccine). The animals to be protected are first inoculated subcutaneously with the greatly attenuated first vaccine and 10 to 12 days later with the less attenuated second vaccine; they suffer only a mild form of the disease and acquire an immunity. Other infectious materials (black leg, pox) can also be treated in a similar manner. 
4. Desiccation. Just as with high degrees of heat, the virulence of some contagions is reduced by drying (withdrawal of water). This method of mitigation has been of practical use in Pasteur's rabies vaccination, also in black leg vaccination (Kitt) and in part in cow pox inoculation (vaccination). In the Pasteur process small pieces of the spinal cord of a rabbit affected with rabies are suspended in bottles and dried; to hasten desiccation the bottom of the bottle is covered with small pieces of caustic potash and at the same time the bottles are held constantly at a temperature of $20^{\circ} \mathrm{C}$. By this method the virulence of the rabies virus is weakened so that after 1 to 2 days' drying 7 days are required for it to produce its effects; after 3 to 5 days' drying, 8 days; after 6 to 9 days', 15 days, etc. As the weakest material is first injected subcutaneously and stronger material at each successive injection, the vaccinated animals (and man) gradually acquire an immunity.

5. The influence of disinfectants. The addition of antiseptics to cultures of bacteria also reduces their virulence. For this purpose carbolic acid, potassium chromate, iodine trichloride, oxygen and other disinfectants have been used in anthrax, tetanus, pox, diphtheria, etc. Sunlight and certain physical factors (high atmospheric pressure) also appear to have an attenuating effect.

6. The progressive inoculation of material of increasing virulence. By inoculating first weakened, then more virulent, and finally infectious material which has not been attenuated, the organism gradually becomes accustomed to the contagion. This process is employed in Pasteur's rabies and anthrax vaccination, in Lorenz's swine erysipelas vaccination (first serum, then culture inoculation), in Koch's vaccination for rinderpest (first bile, then virulent blood), and in other infections. In vaccination for swine erysipelas and rinderpest the weakened and the strong vaccine have been inoculated at the same time (simultaneous inoculation).

7. Long-continued cultivation of the infectious material. As was first shown by Pasteur, the virulence of a pathogenic organ- 
ism decreases with its continued cultivation on artificial culture media. The anthrax bacillus especially progressively decreases in virulence with long-continued cultivation (see Pasteur's vaccination -process) and finally loses its virulence altogether. Similar observations have been made in connection with the contagion of chicken cholera, lung plague, pox and other infectious diseases.

8. The passage of the infectious material through the bodies of animals of another species. The inoculation of some infectious materials into animals of a different species has the effect of modifying their virulence. Best known in this respect is the inoculation of cattle with the virus of pox of man and the reinoculation of man with the considerably attenuated virus thus obtained (cow pox vaccination of Jenner). A similar effect is obtained by the same process with the virus of rabies (monkeys, rabbits), swine erysipelas, tetanus and diphtheria of man (horses, rabbits), anthrax and black leg (sheep, goats, horses) and also in other diseases.

The Different Methods of Vaccination.-According to the part of the body into which the infectious material is injected, the following methods of vaccination are distinguished:

1. The cutaneous or endermatic vaccination consists of a superficial wounding of the epidermis of the skin and exposing the deeper layers of the rete malpighii. By no means must the wound extend to the subcutis. The vaccination is carried out with a vaccination lancet or needle in such a manner as to avoid bleeding if possible. A flow of blood will wash the infectious material off the skin. Cutaneous vaccination is used for foot-and-mouth disease, sheep pox and small pox of man. Corresponding to the cutaneous method is the mucous membrane vaccination, in which there is only a superficial wounding of the epithelium, the submucosa being avoided; cattle are vaccinated for foot-and-mouth disease upon the mucous membrane of the mouth. Vigorous rubbing of the mucous membrane is sufficient to wound it superficially. Corneal vaccination is also included in the term cutaneous. 
2. The subcutaneous or hypodermatic vaccination is carried out with a Pravaz injection syringe or by incising the skin and forming a pocket (mice, guinea-pigs, rabbits, pigeons, chickens). These methods are used for anthrax, black leg, lung plague, swine erysipelas, swine plague and hog cholera, chicken cholera, and rinderpest, in the diagnosis of glanders, for serum inoculation and for the injection of tuberculin and mallein.

3. The interstitial or intramuscular inoculation consists in the injection of the vaccine into the interstitial tissues or muscles. It is therefore an essentially deeper vaccination than the subcutaneous and is employed, for example, for the purpose of diagnosing black leg, rabies, and tuberculosis. Ostertag prefers the intramuscular injection to the intraperitoneal for the bacteriological demonstration of udder tuberculosis (milk) for the reason that the disease can be diagnosed in the inoculated guinea-pigs as early as ten days after the injection (nodules of the size of a pea in the neighboring lymph glands).

4. The intravenous vaccination introduces the infectious material directly into the blood stream. The needle of the syringe is inserted into the vein and the vaccine is injected in the same manner as drugs are injected intravenously. This method is used in the protective vaccination against tuberculosis of cattle (bovovaccine, Tauruman) and was formerly recommended in black leg and lung plague.

5. The intracranial inoculation is used only for the purpose of diagnosing rabies. It consists in trephining the skull-cap of rabbits and introducing the suspected material under the dura by means of a specially constructed curved needle. Analogous methods are the intracerebral (injection into the brain) and the lumbar injection (subdural injection in the lumbar region).

6. The intraperitoneal inoculation has attained a great importance in the diagnosis of tuberculosis. It consists in the injection of suspected milk into the peritoneal cavity of guinea-pigs and is carried out simply by means of an ordinary injection syringe. The result of the inoculation, however, is only to be expected 3 to 
5 weeks after the injection (tuberculosis of the peritoneum and the abdominal organs). This method can also be used in the diagnosis of glanders (guinea-pigs).

7. The intra-ocular inoculation consists in introducing the infectious material by means of a fine injection needle through the cocainized cornea into the anterior chamber of the eye. It is used for the purpose of diagnosing rabies (rabbits, dogs) and also to obtain pure cultures of the tubercle bacillus (rabbits).

8. Galactiferous inoculation is the term used to designate injections through the teat canal into the udder by means of canula-like needles or milk tubes (mastitis bacteria).

9. "Feeding inoculation" is the feeding of infectious material to experimental animals (tuberculosis, anthrax, chicken cholera, swine plague, hog cholera). Intra-intestinal infection is the injection of the material into the intestines.

\section{THE DIFFERENT VARIETIES OF VACCINATION}

Purposes of Vaccination.-While formerly, vaccination was almost exclusively applied to healthy individuals for the purpose of producing immunity to a possible subsequent infection (protective vaccination), it has been more recently employed for other purposes. First of all, mention must be made of the curative vaccination, which has latterly occupied a place in the foreground of therapeutic interest. Its object is to render assistance to the individual in which infection has already taken place (post-infection vaccination), while protective vaccination is employed before infection (pre-infection vaccination). In addition, vaccination is frequently resorted to to establish a diagnosis. This diagnostic vaccination has for its object neither protection nor healing but the production of the disease in question as rapidly and in as definite form as possible, or the generation of fever (tuberculin, mallein) for the purpose of establishing the presence of disease. Accordingly, the following varieties of vaccination may be recognized:

(a) Protective vaccination (pre-infection inoculation).

(b) Curative vaccination (post-infection inoculation).

(c) Diagnostic vaccination. 


\section{Protective Vaccination}

Varieties.-Depending upon how long before the outbreak of an infectious disease the vaccination is made, three varieties of protective vaccination are recognized: protective vaccination in the restricted sense, prophylactic vaccination, and emergency vaccination.

Protective Vaccination in the Restricted Sense.-This is the vaccination of all animals in healthy regions and stables, at a time when the disease is not present, for the purpose of immunizing them against a possible subsequent outbreak of the disease. A good example of this variety of protective vaccination is the vaccination of man against smallpox. In veterinary practice, protective vaccination against sheep pox (ovination) was formerly employed; i.e., all of the sheep in a district were vaccinated although there was no outbreak of the disease. On account of the great danger of introducing, spreading and to a certain extent of artificially breeding the infection when vaccination is applied under these circumstances, this variety of protective vaccination has been abandoned.

Prophylactic Vaccination.-This is also called precautionary vaccination (preventive vaccination) and is used in disease-free stables when the animals are threatened by an infectious disease which has broken out in the neighborhood and which may soon attack them. Prophylactic vaccination is especially important for those regions in which on account of local or agricultural conditions an infection is stationary (anthrax, black leg, lung plague, swine erysipelas, swine plague, rinderpest, sheep pox) and where because of the conditions an effective protection is not to be expected from the operation of veterinary police measures.

Emergency Vaccination.-This is the vaccination of animals which are yet healthy but which are in an infected stable (premises, herd), and is employed most frequently in connection with footand-mouth disease. It is indicated: (1) when the infection of the healthy animals can not be prevented; (2) when it is assured that the artificially inoculated animals will acquire a mild form of 
the disease; (3) when private and general interests will be benefited by the disease passing through the herd as rapidly as possible (shortening the period of continuance of the infection and consequently reducing the restrictions of police regulations and the economical disadvantages). When emergency vaccination is required by law (sheep pox) it is called compulsory vaccination. Permitting the infection of contagious pneumonia and influenza to spread among a lot of horses by not separating the healthy from the sick has an effect similar to that of emergency vaccination.

\section{Veterinary Police Regulations Concerning Vaccination}

The only legal regulations in Germany concerning vaccination refer to lung plague, sheep pox, and swine erysipelas. The German veterinary sanitary law of June 26,1909 , in $\$ 23$, mentions the vaccination of susceptible animals among the measures which may be instituted for protection against infectious diseases. $\$ 51$ provides that vaccination for lung plague can be undertaken only under official direction. $\$ 56$ contains the same restrictions regarding vaccination of sheep for pox and forbids protective vaccination particularly. On the other hand, when the presence of pox is established the vaccination of all animals in the flock is required by $\S 53$ (emergency vaccination). Under $\$ 54$, the vaccination of all flocks threatened by the disease and of all sheep in the same section can be officially required (precautionary vaccination) when sheep pox has obtained a great extension, or when there is danger that it will invade neighboring flocks. $\$ 60$ provides that when swine erysipelas becomes widespread the vaccination of the endangered swine of a herd, of a locality or of a large district may be ordered (precautionary vaccination). It is left with the government to determine when and under what conditions vaccination may be undertaken in other cases.

\section{Protective Vaccination for the Different Animal Infections}

Anthrax. Two methods of vaccination deserve special consideration: Vaccination according to Pasteur with attenuated cultures, and vaccination according to Sobernheim with serum and cultures. 
1. Vaccination According to Pastedr.-The first protective vaccinations were made in 1880 by Toussaint, who heated defibrinated anthrax blood at $50-55^{\circ} \mathrm{C}$. for 10 to 15 minutes and then used it immediately as a vaccine. Pasteur demonstrated in 1881 that immunity could be produced by attenuating the bacilli. Further investigations showed that the virulence of the anthrax bacilli could be reduced by very numerous and different methods: heat (Pasteur, Toussaint, Chauveau), compressed oxygen (Chauveau and Wosnessenski), antiseptics (Chamberland and Roux), sunlight (Arloing). The bacilli can also be weakened by cultivating them upon the blood of vaccinated sheep (Metschnikoff) or in the bodies of frogs (Lubarsch). Pasteur produced his vaccine by cultivating the bacilli at $42-43^{\circ} \mathrm{C}$. in an atmosphere of oxygen for 24 days for the first or weaker vaccine and for only 12 days for the second or stronger vaccine. The animal is first vaccinated with the first vaccine and 10 to 14 days later with the second vaccine. The technique of the vaccination according to the Pasteur method is as follows: The vials sent out from the Pasteur laboratory must be used at once, the entire contents being withdrawn at one time; they must not be opened until immediately before the vaccination. A syringe holding one gram and divided into eight divisions is used to make the injections. Each time before being used the syringe must be carefully cleaned and disinfected. The lymph vial containing the first vaccine is thoroughly shaken, opened and the syringe filled directly from it. One-eighth of the contents is then injected subcutaneously on the inner side of the right thigh of the sheep to be vaccinated, and the opening made by the needle is closed with the thumb. After 12 to 14 days, the second vaccination is made with the second vaccine in the same manner. One-fourth of the contents of the syringe is injected in vaccinating cattle, the first vaccine being injected in back of the right shoulder and the second vaccine in back of the left shoulder; the hair is clipped before the injection is made. Cows in advanced pregnancy and lambs and calves should not be vaccinated.

The results of the Pasteur vaccination are as follows: For sheep it is not to be recommended. Very frequently it produces, especially in sheep, only a very slight immunity or none at all, and in the most favorable cases the immunity lasts only one year, so that it is necessary to vaccinate yearly. Moreover, the mortality resulting from the vaccination is often very great, sometimes amounting to 10 to 15 per cent. after the second vaccination. The Pasteur vaccines are also very inconstant, being at one time too strong and at another too weak, according to whether they have been kept at a temperature nearer to 42 or $43^{\circ} \mathrm{C}$. Sometimes the vaccine shows a return of virulence, especially when it has been attenuated quickly at a high temperature; on the other hand, the virulence may be entirely lost when the vaccine 
is kept too long. Then again, the same vaccine will not answer for all sheep, since the different breeds differ in their susceptibility to the same vaccine. Finally, the cost of vaccination, which is not inconsiderable, must also be taken into account. According to the favorable results obtained in France and Hungary, the vaccination of sheep can only be recommended in an experimental way when a good raccine is available in regions in which the disease is stationary and is attended with regular and considerable losses (at least over 2 per cent.).

For cattle, the Pasteur protective vaccination has a certain value only in badly infected anthrax districts. It is not suitable for use in other sections on account of the uncertainty and short duration of the immunity, the losses resulting and the agricultural disadvantages (illness of vaccinated animals), but especially because of the great danger of the infection of non-vaccinated animals and man. The second vaccine contains strongly virulent bacilli. Horses bear the vaccination very badly.

2. Protective Vaccination According to Sobernheim.-The method of Sobernheim consists of the inoculation of serum followed by the injection of anthrax cultures (simultaneous vaccination); horses and cattle receive on one side of the body 5 c.c. and sheep 4 c.c. of immune serum and five minutes later upon the other side 0.5 c.c. and 0.25 c.c. respectively of an attenuated anthrax bouillon culture of the virulence of the Pasteur second vaccine. In 1904, 75,000 cattle were vaccinated in the Argentine Republic with a loss of only 1 in 1000; up to this time the results in Prussia have been less favorable. On the other hand, curative vaccination with the serum in so far as it has been tested has given very favorable results, corresponding with the results from the earlier intravenous and subcutaneous injection of immune serum.

Lung Plague.-The oldest and most important method of vaccination is that of Willems (1851). The material for inoculation is obtained from an animal in the first stages of the disease. The animal is slaughtered and the diseased areas are removed from the fresh lungs. This diseased tissue is subjected to gentle pressure to express the lymph, which is allowed to coagulate and is then filtered through clean linen. The inoculation is made on the dorsal surface of the tail 8 to $10 \mathrm{~cm}$. from the tip, the injection being made into the subcutaneous tissue in one or two places 1 to $2 \mathrm{~cm}$. apart, after clipping the hair. The lymph must be clear, of a wine-yellow color, and must not be taken from necrotic lungareas, but only from places which are in the stage of hepatization. On the average, the vaccination is effective in 75 to 90 per cent. of the cases. After 1 to 4 weeks there appears at the point of inoculation an inflammatory swelling which is not larger than a hen's egg when the course is normal; in addition, a mild fever and a slight increase in the respiration is observed. 
If the lymph is not entirely pure, there is an extensive swelling of the whole tail with necrosis of the tip, accompanied by very high fever and symptoms of septicæmia and pyæmia. The same effects are produced when the inoculation is made into the dewlap or at the root of the tail, for which reason these places are to be avoided. When the tail becomes highly inflamed, an unfavorable termination must be combated by free incisions into the swelling, antiseptic treatment, and, finally, by amputation of the necrosed end. The deaths always average from 1 to 3 per cent., while 5 to 15 per cent. lose the end of the tail. Sometimes, after 6 to 8 weeks, a second inoculation is made above the point of the first injection.

According to Nocard, cultures of the microörganism of lung plague are better suited for inoculation than the lymph; it is more certain and milder. The vaccine, an 8-day bouillon culture, is injected subcutaneously at the tip of the tail in doses of 0.15 to 0.5 c.c.; the immunity should continue 2 years.

In addition to the caudal vaccination, intravenous vaccination was undertaken by Thiernesse, Defays, Bouley, Degive, Sanderson and others, 2 c.c. of the lymph being injected directly into the jugular. The results were very good and more certain than those from caudal vaccination; in one case inflammation of the lungs, with marmoration, was produced. The vaccination with secondary calf lymph according to Pasteur has not proven satisfactory.

With regard to the value of vaccination, there is no agreement. The advocates of vaccination base their claims upon the well-known fact that after recovering from an attack of the disease cattle are immune for life. Vaccination sets up a specific local inflammatory process exactly similar to that which takes place in the lungs in lung plague and generates an immunity of the entire body. In addition, vaccination shortens the course of the plague in a stable. The losses after caudal vaccination are declared to be entirely inconsiderable. Haubner calculates that 1 to 2 per cent. die, while 5 to 10 per cent. lose the end of the tail. In Holland, where 60,000 cattle were vaccinated in 1878-79, the mortality amounted to 0.66 per cent. The favorable results obtained in the Spöling district of Holland, in the province of Saxony, in the duchy of Anhalt and in Australia are presented as proof of the value of vaccination. To these are added the positive results obtained by Schütz and Steffen. A further extension of the disease through vaccination is denied. Moreover, compared with the great pecuniary losses attending the slaughter method of controlling the disease, vaccination is by far the cheapest; while the value of sanitary laws, however stringent, is disputed. The following statistics are presented: Degive computed the results of vaccination in several countries from 1850 to 1883 and found that of 6705 vaccinated animals only 2.7 per cent. contracted the disease, and that of 2453 non-vaccinated animals 26.9 per cent. became infected, although both classes of animals were similarly 
exposed to the disease. According to Pütz, the number of animals affected with lung plague in Holland was reduced by vaccination from 6079 in 1871 to 2227 in 1875, 951 in 1877, 157 in 1879 and, finally, 11 in 1882. In Hasselt, where 200,000 cattle were vaccinated with good results during the period from 1850 to 1880, the losses regularly increased when vaccination was omitted. Rochebrune relates that for years the Moors in Senegambia have vaccinated their cattle against lung plague with good results by sticking the point of a knife into the lungs of a slaughtered animal and then incising with the knife the skin of the animal to be vaccinated in the region of the nostrils.

The opponents of vaccination point out that a positive case of immunization by vaccination has not been demonstrated up to this time. Even the friends of vaccination are not in a position to state how long the immunity continues, whether $1 / 4$ or 1 or 2 years; others who favor vaccination speak only of partial action and immunization and therefore vaccinate several times. The specific character of the vaccination swelling is disputed, since an entirely similar swelling arises after the inoculation of pus or milk. Furthermore, the vaccination has in no case produced a lung plague pneumonia, the principal criterion of the disease, while this alteration occurs even in those cases in which the infection is transmitted intra-uterine from the mother to the foetus. The results of vaccination are dependent upon the method and time of inoculation and upon the quality of the vaccine. Animals already immune were also frequently vaccinated and the previously acquired immunity attributed to the effects of the vaccine. The disease has been introduced and artificially propagated by vaccination. The losses from vaccination are under certain circumstances very considerable; the mortality isfrequently very great, even at times exceeding the usual fatalities from the disease itself. In the preamble to the German Imperial veterinarysanitarylaws thelosses from vaccination are given as 2 to 4 per cent.; the end of the tail is lost in 25 per cent. of thecases according to the observations of a French vaccination commission, in 10 to 15 per cent. according to Degive. In addition there are the economical disadvantages, decrease of the milk secretions, emaciation, etc. The disease of ten spreads in spite of vaccination, while in other cases it subsides without vaccination; many animals have the disease without showing perceptible symptoms. In those countries in which vaccination was most extensively practised the disease is not yet on the decline. In England, for example, and in France and Belgium, in which vaccination was obligatory, as well as in the province of Saxony, the disease still exists. In other countries, for instance Holland, the repression of the disease is perhaps not the result of vaccination but of the simultaneous enforcement of veterinary police measures, especially slaughter. In Belgium, according to Oemler, the number of cases of lung plague increased from 1481 in 1867 to 2800 in 1878 in spite of vaccination, but after that date, 
in consequence of the strict enforcement of veterinary police regulations, the number of cases rapidly decreased, until in 1880 there were 1781 and in 1883, 1187 cases. A compilation made by Kitt shows that in England the number of cases decreased only with the adoption of stringent protective regulations in 1878 from 4590 to 2144 in 1879 and to 1200 in 1882 . In Bavaria, following the introduction of the Imperial veterinary sanitary laws, the number of cases decreased from 846 in 1846 to 281 in 1883. In Baden, where from 1870 to 18800.2 per cent. of all the cattle died yearly from lung plague, there was not a single case in 1885 .

The answer to the question as to the value of vaccination for lung plague depends principally upon whether it is to be used only for immunizing individual animals or to combat an outbreak of the disease in a herd or in a district. In this connection the following statements can be made: (1) Vaccination, as a rule, confers a high degree of immunity upon iadividual cattle. (2) The value of vaccination as a veterinary police measure for combating lung plague is, on the contrary, doubtful. Experience has many times taught that not all vaccinated cattle acquire an immunity, but that about 2 per cent. remain susceptible to the disease. These apparently immune cattle, when they subsequently suffer a latent attaels of the disease, are a continuous source of infection for healthy animals, especially in regions where the traffic in cattle is extensive (province of Saxony). Furthermore, lung plague sometimes runs such a mild course that no symptoms of disease are apparent and the recognition of the disease in time for the vaccination of the herd is not possible. The length of the period of incubation also often prevents a prompt diagnosis or early vaccination. As a method of combating the disease, slaughter of the infected herds is much more reliable than vaccination.

Rabies.-Pasteur announced in 1884 that the virus of rabies could be gradually weakened by inoculating it from the dog into a monkey and then successively from monkey to monkey. Virus attenuated in this way and injected subcutaneously or under the dura mater, after trepanation of the cranial cavity, does not produce rabies in the dog, but on the contrary renders the animal immune to artificial or natural infection with the disease. Subsequently, Pasteur published another method of immunization in which the dried spinal cord of a rabid animal is used as the vaccine. The spinal cord of a rabbit affected with rabies is removed under aseptic precautions up to the lower end of the cerebellum, together with all of its coverings, and cut into pieces $6 \mathrm{~cm}$. long, which are suspended on threads in bottles. The bottoms of the bottles are covered to a depth of $1.5 \mathrm{~cm}$. with small pieces of caustic potash and their openings are closed with cotton plugs. They are kept at a constant temperature of $20^{\circ} \mathrm{C}$. After 3 to 4 days, the pieces of spinal cord are dried into ribbon-like, very friable strips, the toxicity of which gradually 
decreases with the continuance of the drying. For example, rabbits develop rabies after 7 days when they are inoculated with material which has been dried 24 to 28 hours; after 8 days, when the material has been dried 3 to 5 days; after 15 days, when the drying has continued 6 to 9 days. Animals (horses, dogs) and man are made immune to rabies by injecting them first with a very weak virus, then with a slightly more virulent virus, and so on, until at last a very strong virus is injected. After this, the full strength rabies virus can be injected without harm. This method of vaccination has proven of value also as a curative remedy when applied to human individuals bitten by rabid dogs (post-infection vaccination). Other methods of vaccination for rabies (diluted virus, intravenous injection of brain substance, serum inoculation, simultaneous vaccination) have been published by Högyes, Helman, Babes, Galtier, Protopopoff, and others.

Foot-and-mouth Disease. - As early as the beginning of the nineteenth century (Buniva), and very frequently since then, vaccination in the form of emergency vaccination has been effectively employed to induce the regular extension and to shorten the course of foot-and-mouth disease in large herds (Ercolani, Brauell, Renner, Hoffmann, Wirtz, Spinola, Hertwig, Lewes, Brandes and others). Emergency vaccination is a measure entirely worthy of recommendation, because it not only causes a more rapid extension of the disease through a herd and consequently permits an earlier removal of the sanitary police regulations, but the vaccination disease frequently runs a milder course and is confined to the mouth. The emergency vaccinations made in the last ten years in numerous herds in almost all sections of Germany have mostly been accompanied by good results; their influence was most favorable when the vaccinations were made at the first appearance of the disease. Of the vaccinated animals, 50 to 80 per cent. became infected on the average; the others proved themselves to be immune. Emergency vaccination is only contraindicated in the malignant form of foot-and-mouth disease, in which the vaccination disease may be dangerous.

The technique of the vaccination is very simple. The saliva of an affected animal is placed in the mouth of the animal to be vaccinated at a point where the mucous membrane has been previously rubbed, or it is inoculated with a lancet anywhere in the skin; impregnated threads can also be drawn under the skin of the ear or tail. Swine are inoculated on the nose with a syringe and needle. Fever occurs 24 hours after the inoculation; on the third day the vesicles appear, and healing of the ulcers begins from the sixth day on. The course of the vaccination disease is in general milder than that of the natural infection.

With reference to the protective vaccination with blood-serum, the investigations are not yet concluded. Hecker's vaccine and "seraphthin," intro- 
duced by Löffler in 1899, did not answer the purpose. It is doubtful if the sera employed by Nocard and Löfler on cattle ( 2 to 3 weeks' immunity), sheep and swine (3 to 8 weeks' immunity) will prove of practical value. A method of protective vaccination suitable for practice is not yet known. Löffler's serum (1912) is not adapted to general use because of the high price (about $\$ 7$ per animal), the complicated process (four vaccinations) and the short duration of the effect.

Sheep Pox.-In the first half of the nineteenth century, entire flocks of sheep were subjected to protective vaccination against sheep pox (ovination) each year, with the assistance of special vaccination institutes, whether an outbreak of the disease was threatened or not. This regulation proved to be very objectionable. Not only did the protective vaccinations establish permanently infected flocks, in which the disease became stationary, but the iufection spread from these flocks to the adjoining regions, the danger of healthy animals being infected by vaccinated individuals being exceedingly great. In several countries, for example in Prussia and Austria, the spread of the disease went hand in hand with the protective vaccination. On the other hand, emergency vaccination is worthy of recommendation, and is therefore included in the requirements of the German sanitary laws ( $\$ 53$ ). Emergency vaccination is employed only in those flocks in which the disease has already broken out. Not only does it cause the disease to run a quicker course, inducing a more rapid extension of the infection through the flock and consequently an earlier removal of the sanitary police restrictions, but the disease itself is of a milder type and usually only local, so that frequently there are no deaths and usually not more than 2 per cent. die. Only when all external factors are unfavorable, the losses exceptionally reach 10 per cent. In addition to emergency vaccination, precautionary or prophylactic vaccination may also be employed when an extensive outbreak of sheep pox prevails in the neighborhood of healthy flocks and the situation is such that there is great danger of these flocks being infected ( $\$ 54)$.

The vaccine, or "ovine," can only be obtained from sheep with normally developed pox and in which the disease runs a benign course. The lymph must be entirely clear and limpid, neither turbid nor purulent; it is therefore usually collected from the vaccinated sheep on the 10th to the 12th day after the vaccination, or 6 to 8 days after the eruption. The vaccinated sheep must be kept separate from the sheep from which it was inoculated, and the sheep to be vaccinated must not be permitted to come in contact with the infected isheep from which the material for inoculation is obtained, otherwise infection may take place spontaneously at the same time. The inoculation may be made on the inner surface of the ear, $4 \mathrm{~cm}$. from the tip, or better on the under surface of the tail, 10 to $12 \mathrm{~cm}$. from the anus. In the latter case the animal 
must be laid down. Vaccination on the ear is not entirely without danger on account of the proximity of the eye. The instrument used is either a fine and pointed vaccinating needle with a spoon-like excavation, or a vaccination lancet. In vaccinating an entire flock, it is an advantage, when time will permit, to make a preliminary or test vaccination of six to twelve animals. The vaccinated sheep show an entirely regular pox exanthema which is limited to the point of vaccination, and very mild general symptoms. The pox become ripe on the tenth day after vaccination. Exceptionally, the pox do not develop at the point of vaccination but in the surrounding area (accessory pox); very rarely, a general eruption of pox is observed following the vaccination eruption (secondary pox). The after-treatment consists in protecting the vaccinated animals from unfavorable weather and providing them with suitable food. In addition, an examination of the flock should be made in ten to twelve days and those animals again vaccinated in which the first vaccination was not effective.

In place of ovination, serum vaccination, serum therapy and simultaneous inoculation with immune serum and virulent lymph are also recommended (France, Roumania).

Rinderpest.-1. The OtD Stbcutaneods Metrod.-On the steppes of Russia, where this form of vaccination was practised as early as the middle of the eighteenth century, it was formerly employed as a prophylactic measure; but only in the form of emergency and precautionary vaccination, never as protective vaccination, because of the great risk of spreading the disease by the latter. At the present time there are four vaccination institutes in Russia (Kharkov, Karlovka in Poltava, Bondarewka in Kherson, and Salmysch in the Orenburg government), but vaccination is falling more and more into disuse. In Germany and the other European countries, except Russia, emergency vaccination is not permitted, because in them veterinary police measures, i.e., slaughter, are much more effective and certain and because the mortality from the vaccination of ordinary cattle is much too high. The mortality among the cattle of the steppes from vaccination is only about 10 per cent., while among the other breeds it is 36 per cent. Toward the end of the outbreak favorable results from vaccination are relatively more frequent. The technique of the vaccination is simple. A clean sponge is placed in the nostril and permitted to remain there until it has absorbed its fill of nasal mucus, when it is removed and the contents expressed into a small glass vessel, which is closed. A drop of this material is then injected under the skin of the neck with a Sticker syringe.

2. Bile Inoculation According to Koch,-During his investigations into the cause of rinderpest in South Africa (Kimberley) in 1897, Koch found that the bile as well as the blood-serum of cattle that had passed through an 
attack of the disease possessed immunizing properties. The bile vaccination consists simply of a single subcutaneous injection into healthy cattle of bile (10 c.c.) from cattle affected with rinderpest or which have passed through an attack of the disease. Six to 10 days after vaccination, an immunity is established which continues 4 to 6 months. The local effect consista of a hard swelling of the size of the fist, which disappears after a few weeks. Protective vaccination with bile is of extraordinary value in infected regions. Kohlstock, who introduced Koch's method of vaccination into German Southwest Africa, recommends a double vaccination of bile and rinderpest blood (emergency vaccination). This double vaccination was later approved by Koch. According to Kolle, Koch's bile method has the disadvantage that the animal is susceptible to infection for a whole week after vaccination. Furthermore, it is necessary to slaughter 3 to 7 diseased or recovered animals to obtain suffcient bile to vaccinate 100 cattle. The mixture of bile and glycerin proposed by Edington is without value, as the addition of the glycerin reduces the degree as well as the continuance of the immunity. According to Hutcheon, Koch's bile vaccination method has been abandoned entirely in Cape Colony on account of its great imperfection.

3. Sertom Vaccination.-This method requires 100 c.c. of blood-serum and produces only a passive immunity of short duration. It may be used to separate an infected region from a rinderpest-free region by creating a broad immunized area around the infected region. It has preference over the bile vaccination in that it is effective in the incubation period of rinderpest.

4. Simultantous Vaccination.-Kolle and Turner recommend the simultaneous injection of 1 c.c. of virulent rinderpest blood upon one side of the body and the injection of 15 to 40 c.c. of serum upon the other. This method confers an immunity for five months. Koch and Theiler have raised the objection against the simultaneous method that piroplasmæ (infectious hæmoglobinuria) and trypanosomes may be inoculated with the rinderpest blood in regions infected with these parasites.

Black Leg.-1. Tye Lxons Method of vaccination was discovered by Arloing, Cornevin, and Thomas. In 1880 and 1885 they vaccinated intravenously, but with great losses. They therefore later selected the subcutis at the end of the tail as the point of vaccination, because they had found that the black leg virus when introduced here produced only a temporary harmless swelling but nevertheless established subsequently a complete immunity. The slight reaction at this point is explained, in their opinion, by the density of the connective tissue and the low temperature existing there, both of which conditions prevent the spread of the black leg bacilli through the connective tissue spaces. In addition, they also weakened the black leg virus before injection by exposing it to high degrees of temperature. The preparation of 
the vaccine and the process of vaccination according to Arloing's method (Lyons method) is as follows: 40 grams (1 part) of diseased muscle tiseue is quickly dried at $33^{\circ} \mathrm{C}$. and uniformly mixed with 80 grams (2 parts) of water. The entire amount is then divided into 12 parts of 10 grams each, each part is placed in a suitable flat dish and dried for 6 hours in a thermostat. Onehalf is kept at a temperature of $100^{\circ} \mathrm{C}$. in order to obtain the weaker-acting first vaccine, and the other half is exposed to a temperature of $85^{\circ} \mathrm{C}$. to produce the stronger second vaccine. The vaccination is carried out in two sections, the first with the weaker and the second with the stronger vaccine. The dried brownish crust in the plates is used in the vaccination; this material can also be stored for a long time. For the first vaccination, 0.1 gram of the material which was heated at $100^{\circ} \mathrm{C}$. is triturated in a disinfected mortar with 5 grams of water, the contents of a 5-gram Pravaz syringe. The mixture is then filtered through a clean piece of linen and the filtrate is drawn up into the syringe, the piston of which is marked off in half-cubic centimetres and provided with a movable disk to be used in measuring the dose. The quantity of the vaccine injected into each animal is 0.5 c.c., so that the syringe will hold sufficient for 10 animals.

The point of the first vaccination is the under surface of the tail, three hand-breadths from the tip. After the hair is clipped, the trocar which accompanies the syringe is inserted obliquely through the skin and passed upwards between the skin and bones about $8 \mathrm{~cm}$.; it is then removed, the canula of the syringe introduced through the same wound and the vaccine, after being mixed by shaking the syringe, is slowly injected. To prevent the vaccine from flowing out, the point of entrance is compressed and the injected fluid is pressed upward with the thumb. If any bleeding occurs, the injection is delayed until the hemorrhage is checked, or another place is chosen. Finally, a rubber bandage $2 \mathrm{~cm}$. in width is placed upon the point of injection and allowed to remain four hours for the purpose of preventing the escape of the vaccine. In this way, it is said, 20 to 25 animals can be vaccinated in an hour, the animals being held by three assigtants. Ten days after the first vaccination, the animals are vaccinated with the second or atronger vaccine. The second vaccination is carried out in the same manner as the first except that the injection is made only two hand-breadths from the tip. The most favorable season for vaccination is spring, or the close of winter. The vaccination is not followed by any sequelæ. The vaccination experiments carried out in different countries (France, Switzerland, Austria, Germany) have established that the Lyons method of protective vaccination undoubtedly reduces the mortality from black leg very considerably.

2. The MUNich METhoD of vaccination, or Kitt's method, consists of a single subcutaneous injection on the under surface of the tail. The vaccine 
solution is prepared from powdered muscle which has been attenuated by exposure to live steam at $97^{\circ} \mathrm{C}$. (or by heating for six hours at 85 to $90^{\circ} \mathrm{C}$.). More recently, pieces of muscle of different origin are used in preparing the vaccine (polyvalent vaccine).

3. Tam Biacklegine of Thomas. - Silk or cotton threads containing black leg spores are inserted subcutaneously (back of the shoulder in calves) and permitted to remain. The method is recommended as very simple and effective and has given satisfactory results in Germany (North Dithmarsch).

Swine Erysipelas.-1. The Lorenz Metrod of vaccination and the analogous Susserin consists in the simultaneous injection of immune serum (passive immunization) and bouillon cultures of swine erysipelas bacilli (active immunization). The inoculation is made at the base of the ear, where 5 c.c. of serum and 0.5 c.c. of the culture are injected subcutaneously at the same time (simultaneous vaccination). The immunity established by the vaccination continues about half a year. Simultaneous vaccination has proven very trustworthy in Germany as a protective and curative remedy. Recently, instead of the double inoculation only the serum is injected in many instances.

2. The PABTEUR Method comprises twoinjections of attenuated cultures of swine erysipelas bacilli (premier and deuxième vaccine) on the inner surface of the thigh at intervals of 10 to 12 days; the first the weaker and then the stronger vaccine (attenuated by passing through rabbits). This method established a high and long-continuing immunity, but is not without danger (extension of the disease, sometimes great losses).

Hog Cholera.-By the use of 10 to 20 c.c. of immune serum, obtained from swine highly immunized artificially, a passive immunity is established which begins during the period of incubation and lasts several weeks. The immune serum also has a curative effect on diseased animals. By the simultaneous injection of immune serum (20 c.c.) and virulent blood (1 to 2 c.c.) an active immunity is produced which lasts for months; this method, however, appears to be not without danger, because a part of the vaccinated swine become affected with hog cholera and eliminate the virus of the disease.

[In the United States, 20 c.c. of serum are injected for each 50 pounds of body weight both in the serum alone and in the simultaneous method of vaccination.]

Swine Plague.-The several vaccines recommended for swine plague (Septizidin, Suptol, Euman, bivalent and polyvalent serum) have not proven reliable in practice.

Calf Pneumonia.-The views concerning the value of the different immune sera (Septizidin, polyvalent serum) are divided and contradictory.

Calf Cholera.-The serum vaccination of new-born calves with polyv- 
alent coli serum (Jensen) is effective, but the protective vaccination of cows in advanced pregnancy has, on the contrary, proven to be without value.

Fowl Cholera. - 1. Protective Vaccination with Atrendated ColTuRes According to PAsteur.-In 1880, Pasteur recommended the vaccination of healthy individuals with cholera bacilli attenuated by cultivation as the most effective method of combating fowl cholera. He found that vaccination with a weakened (mitigated) vaccine produced only a local swelling at the point of inoculation and that the muscle tissue beneath underwent necrosis without suppuration. The vaccinated bird was sick, indeed, but did not die and became immune to the disease. Some chickens, however, required 2 to 3 vaccinations with mitigated vaccine before they acquired an immunity. Pasteur therefore introduced a double vaccination, first injecting a very weak vaccine (premier vaccine) and then a stronger one (deuxième vaccine). The bacilli were attenuated by keeping them for 3 to 10 months under conditions which permitted the entrance of air, whereby, according to Pasteur's theory, their virulence was reduced by the oxygen of the air. Protective vaccinations were made according to this method by Cagny, Hess, and Kitt, and the results were very unfavorable (spread of the disease, fatal cases).

2. Sendm Vaccination has been of doubtful value in Prussia, but in Denmark very favorable results have been obtained.

Contagious Pneumonia of Horses.-The first serum vaccinations were made by Hell in 1892 . The vaccinations made the same year in the Prussian army to test the new process were, however, without effect, since the disease occurred with the same intensity in the vaccinated horses as in those which had not been vaccinated, while many horses which were not vaccinated did not take the disease (P. Mil. V. B., 1892). The reports concerning the vaccinations made in the army with blood-serum in 1893 are very contradictory (P. Mil. V. B., 1893). In 1894, vaccinations were made in four regiments. The results did not speak favorably for the vaccination; it neither shortened the course of the disease nor reduced the virulence. The isolated favorable results were only apparent and are to be explained by the benign character of the infection (P. Mil. V. B., 1894). In 1895 the serum vaccinations were also without effect, although every horse received 200 grams of serum in four days; 10 weeks after the vaccinations 10 horses in one squadron became ill with contagious pneumonia, some being very severely affected (P. Mil. V. B., 1895). On account of these results the vaccinations were discontinued in the army in 1896. In 1898 Töpper made a report of his experiences with bloodserum vaccinations and stated that the horses became immune 6 to 8 weeks after the injection of the serum. He also stated that the disease usually prevailed in the stable 4 to 6 weeks before the vaccinations were begun and that after the vaccinations it was always immediately checked. Not less than 150 
grams of blood-serum must be injected and it must be obtained from horses in the same stable which had recovered from the disease and which had been free from fever for at least 3 to 6 weeks. As Töpper did not make his vaccinations until 4 to 6 weeks after the appearance of the disease, Schwarznecker pointed out that it was very questionable whether the cessation of the outbreak was due to the vaccinations or to the natural course of the epizoötic. The Prussian military report for 1898 shows that the protective vaccination with blood-serum is of no practical value. Contagious pneumonia did not prevail as long in those divisions of the army in which no vaccinations were made as in those in which the horses were vaccinated. In the winter of 189899, Tröster made serum injections in 17 batteries and 6 squadrons; each horse receiving 50 grams of serum. The vaccinations caused an immediate pause of 5 to 36 days in the progress of the epizoötic, but the effect did not last and was insufficient, since the epizoötic was not extinguished. This brief protection, according to Tröster, is only of value in the case of a mobilization, when it may be desirable to protect a large number of horses from infection for a a few days. All attempts to cure horses affected with contagious pneumonia by vaccination with serum have failed. Further experiments were made by Tröster in 1899 on 58 horses with a larger quantity of serum (500 c.c., in two portions, injected into the breast). The results of this experiment, by no means extensive, seemed to Tröster to justify the conclusion that the injection of such large doses produced asufficient immunity, although the amount of serum required was very difficult to obtain. In 1900 and 1901, he vaccinated 784 and 635 horses respectively with 100 to 150 grams of active blood-serum; some of the vaccinations were effective (40 days immunity) and some were not. In May, 1900, 518 horses in Dragoon Regiment No. 21 were raccinated by him and no effect was observed on the spread of the disease; 195 of the vaccinated horses developed extensive swellings and abscesses and some of them were not fit for service for 14 days. Mieckley vaccinated 200 horses with 200 grams of serum each in the Beberbeck stud in 1900. The results were negative. In spite of the vaccination, 113 horses became affected with contagious pneumonia, and of these 14 died.

In the light of these experiences there can remain no doubt that serum vaccination for contagious pneumonia is of no value.

Cow Pox Vaccination of Man (Vaccination). -Since 1796 the lymph of cow pox (vaccine) has been used for the protective vaccination of man against smallpox (variola). Three kinds of vaccine are recognized: original, human, and animal. The original vaccine is the lymph obtained from natural cow pox, which was used in the beginning of vaccination; the human vaccine is obtained from cow pox produced artificially on children, and the animal vaccine is obtained from calves. At the present time, almost all vaccinations 
are made with animal vaccine. The animal lymph is obtained in the following manner: Healthy calves (especially steer.calves) one-fourth to one-half year old, which have passed the tuberculin test, are inoculated with human vaccine in special vaccine institutions (100 to 200 punctures or crucial incisions upon the shaved and cleaned skin of the abdomen). On the fifth day the lymph is collected from the pox which have developed with special instruments (clamp forceps, capillary tubes, lancets, spatulæ, glass plates) and conserved (exclusion of air in glass tubes, glycerin, thymol, salicylic acid, drying). The principal advantage of animal over-human lymph is the certain avoidance of the transmission of disease from the vaccinated children to those subsequently vaccinated (ayphilis, tuberculosis, acute exanthemas). The immunity established by vaccination continues for about ten years; after this time a re-vaccination is necessary.

Tuberculosis.-For the protective vaccination of cattle against tuberculosis von Behring, in 1902, recommended the intravenous injection of living human tubercle bacilli into calves 3 to 6 weeks old (Jennerization, Bovovaccine). First, 0.004 gram of tubercle bacilli suspended in 4 grams of water is injected into the jugular vein, and later 25 times the quantity of tubercle bacilli, $0.01 \mathrm{gram}$, suspended in 4 grams of water, is injected. [In 1902, Pearson and Gilliland published a report of their experiments in which they had demonstrated that it was possible to immunize cattle against tuberculosis (Phila. Med. Journal, Nov. 2, 1902). These experiments were begun September 29, 1900. They used living tubercle bacilli of the human type, injecting 0.004 of dried. tubercle bacilli suspended in salt solution intravenously and four weeks later twice this quantity.] A similar method of vaccination was recommended by Koch and Schütz (Tauruman), who claimed that a single intravenous injection of 0.01 to 0.03 gram of tubercle bacilli from human sources or of attenuated bacilli from cattle would immunize cattle against highly-virulent tubercle bacilli of bovine origin. These methods of protective vaccination have not proven of practical value because only a temporary immunity is established. The use of vaccine material containing virulent tubercle bacilli is also attended with several disadvantages: the infection of the vaccinated calves with tuberculosis, the infectiousness of the meat and milk of the vaccinated animals for man (excretion of virulent tubercle bacilli in the milk), the danger of the vaccination to the veterinarian, the transformation of chronic calf pneumonia into the acute form, and other dangerous vaccination accidents (embolic pneumonia, apoplectic death, severe febrile disease, emaciation, etc.). Antiphymatol and tuberculosan have proven equally inefficient.

Strangles.-The serum treatment of strangles (protective vaccination, curative vaccination) has not proven reliable in Germany. Experience with 
the different preparations of serum has been unsatisfactory, especially in the Prussian remount depots and in east Prussia. In Denmark, on the other hand, the protective action of subcutaneous injections of dead strangles cocci [bacterin] and the curative effect of polyvalent serum have attained a great reputation. Fröhner's experience with the Danish serum confirms the reports of its curative value. Protective vaccination (active immunization with cocci) has been recently recommended in Germany.

\section{Corative Vaccination}

Character.-While by protective vaccination a certain time before the entrance of infection an immunity is obtained (preinfection vaccination), the purpose of curative vaccination (postinfection vaccination) is to accomplish the same result after infection has taken place. The first attempt to heal an already infected body by vaccination was made by Pasteur in his experiments in the cure of rabies (1885). Then followed the experiments of Koch, von Behring, [Pearson and Gilliland], Kitasato and others with tuberculosis, tetanus, diphtheria, pneumonia, anthrax, swine erysipelas, etc.

Curative Vaccination in Rabies.-According to Pasteur, human beings who have been bitten and infected with rabies may be subsequently immunized by vaccination if they are inoculated in a systematic manner with attenuated virus. A piece of the dried spinal cord of a rabbit 2 to $3 \mathrm{~cm}$. long (see p. 242) is rubbed up with sterile bouillon, forming an emulsion, of which $1 / 2$ to $3 / 4$ c.c. is immediately injected under the skin of the abdomen. In the beginning a very weak vaccine is injected, and at each succeeding injection a somewhat stronger vaccine is used. Pasteur's work was confirmed by Contani, Metschnikoff, Ullmann, Bujwid, Bardach, R. Koch and others. It was not accepted, however, by von Frisch, Högyes, de Renzi, Bordoni-Uffreduzzi and others.

Curative Vaccination in Swine Erysipelas.-In the use of swine erysipelas serum in protective vaccination, very many cases have been observed in which swine affected with erysipelas have apparently been cured when the serum was injected early, 6 to 12 hours after the appearance of the first symptom, and in large (10 to 30 c.c.) and repeated doses. 
Curative Vaccination in Tetanus.-The curative action of tetanus antitoxin in tetanus of man was discovered by von Behring in 1896. Since that time it has been frequently employed in veterinary medicine in tetanus of horses. At first its action as a curative agent appeared to be favorable, but later the failures increased to such an extent that it is now used only in isolated cases. In the Prussian army, from 1896 to 1907, 129 horses affected with tetanus were treated with the antitoxin and 85 , or 66 per cent., died. During the years previous to the use of the serum the mortality was as follows: in 1881, 55 per cent.; 1882, 57 per cent.; 1884, 65 per cent.; 1885, 62 per cent.; 1887, 67 per cent.; 1888, 66 per cent.; 1892, 65 per cent. In the light of these figures it cannot be said that the use of the antitoxin in the years from 1896 to 1907 exerted a favorable influence upon the disease.

Recently, it was announced that the preparations of antitoxin heretofore used were too weak and that the material had been employed in insufficient quantity. It was also stated that the antitoxin must be injected as early as possible, immediately after the diagnosis is made, preferably intravenously, and the injections repeated until improvement occurs. Sawamura (Bern, 1909), in experiments with rabbits affected with tetanus ascendens endoneural, found that their life was saved when the antitoxin was injected not later than 17 hours after the appearance of the first tetanic symptom. In order to test the value of this new antitoxin, Fröhner obtained 500 c.c. of it from Marburg in the summer of 1910. The cost of the 500 c.c. was twenty-five dollars, five dollars per dose (100 c.c.). Without selection, the first two cases of tetanus entered in his clinic were treated with the antitoxin according to the accompanying directions, while the third and fourth cases received no treatment. Both of the horses which received the antitoxin immediately after the diagnosis was made in the first stages of the disease died, while the other two, which received no antitoxin, recovered. From this it would appear that the new antitoxin is not a reliable curative agent in tetanus. 
Curative Vaccination in Hog Cholera.-The serum inoculation appears to have been proven to be also a curative method (see p. 248).

Curative Vaccination in Tuberculosis.-Tuberculin, which was recommended by Koch as a specific curative agent in tuberculosis in man, did not prove to be effective for this purpose. Furthermore, it is of no value as a curative agent in tuberculosis of cattle.

Curative Vaccination in Septicæmia.-Several sera are recommended as curative and protective remedies in the diseases caused by streptococci and for the complications of contagious pneumonia; e.g., the anti-streptococcic serum of Marmorek and the sera against purpura hemorrhagica of Lignieres and Jensen. The reports concerning the action of these sera are contradictory. The Danish polyvalent serum against purpura hemorrhagica (Jensen) proved effective in a case treated by Fröhner.

Organotherapy.-The terms organotherapy, tissue-fluid therapy, and opotherapy are used to designate the use of animal organs as curative agents. Testicles, thyroid glands, ovaries, prostates, liver, etc., as such, or in the form of special preparations (spermin, thyroidin, iodothyrin, etc.), are used in impotence (spermin), goitre and myxœedema (thyroidin, iodothyrin) and diseases of the ovaries, prostates, liver, etc., being administered internally. Only the preparations of the thyroid gland in goitre and myxcedema appear to have an undisputed action. Experiments in Germany with the other opotherapeutic preparations have usually resulted negatively.

\section{Diagnostic Inoculation}

Purpose.-The object of diagnostic inoculation is to ascertain experimentally the identity of the disease in cases in which a certain demonstration and positive recognition of the infection is not possible with the usual clinical methods. This is accomplished by artificially inoculating healthy experimental animals with infected masses (blood, nasal discharge, vaginal discharge, pus, milk, fæces) in order to produce a typical picture of the disease (inoculation of other animals), or by inoculating the originallydiseased animal (self-vaccination, auto-inoculation). In a certain 
sense, injections of tuberculin and mallein belong to the latter method of inoculation.

Inoculation of Other Animals. - This method of inoculation is used for the purpose of diagnosing anthrax, glanders, tuberculosis, fowl cholera, swine erysipelas, hog cholera, swine plague, strangles, contagious pneumonia of horses, hemorrhagic septicæmia, black leg, rabies, malignant œdema and Canadian horse pox.

1. Anthrax.-Although the protective vaccination for anthrax is subcutaneous, the diagnostic inoculation is made cutaneously in order to avoid mixed infection. The best animals for inoculation are mice, rabbits and guinea-pigs; the best inoculation materials are the blood and spleen pulp. Mice are inoculated on the end of the ear after the tip has been clipped off; rabbits and guinea-pigs are inoculated in slight scratch-wounds on the ears. If anthrax is present, the inoculated animal dies in two to three days; on post-mortem examination numerous characteristic anthrax bacilli are found in the blood.

2. BLACK LEG.-Guinea-pigs are used as inoculation animals. Rabbits are immune to black leg but are very susceptible to anthrax. If guinea-pigs and rabbits are inoculated simultaneously with the same material and only the guinea-pigs die, then black leg is probably present. Inoculated guinea-pigs continue to live if they are treated with black-leg serum. In contrast with anthrax, the inoculations are made subcutaneously only and not cutaneously. The subcutaneous inoculation of guinea-pigs is the most important diagnostic method in doubtful cases and in questionable post-mortem findings.

3. GLANDERs. - Several male guinea-pigs are inoculated simultaneously with the suspected material (pus). The inoculations are made subcutaneously in the abdominal region - a fold of skin is cut with small scissors and a pocket is formed into which the material is inserted; or, the material is injected intraperitoneally. Fourteen days after the subcutaneous injection, if glanders is present, characteristic symptoms appear. The point of inoculation has been transformed into an ulcer, in the proximity of which 
nodular swellings of the lymph glands up to the size of a hazelnut may be felt; later, these rupture; on post-mortem examination, purulent lymph glands and glanders abscesses are found, the latter especially in the testicles (glandular orchitis); also glandular nodules in the spleen, liver and lungs. A negative result from the inoculation does not exclude the presence of glanders. When the result is positive, a diagnosis of glanders is only justified when the bacillus mallei is also demonstrated in cultures (pseudo-glanders!). Other inoculation animals are the horse and the ass, the latter contracting acute glanders and usually dying in about 8 days; also cats, which, after subcutaneous (dorsal) inoculation, present symptoms similar to those exhibited by guinea-pigs. Concerning injection with mallein, see page 260 .

4. Tuberculosis.-The best inoculation animal is the guineapig. After the usual intraperitoneal inoculation (milk), numerous fresh tubercles of the size of millet seed appear in about three weeks upon the peritoneum and in the spleen, liver, and lymph glands. Following subcutaneous injection (mucus from the lungs), a purulent ulcer develops at the point of injection and the neighboring lymph glands become swollen; after 3 to 4 weeks numerous tubercles form in the internal organs. After intramuscular inoculation (rapid inoculation), the neighboring lymph glands are transformed in ten days into firm nodules of the size of small peas, which may be extirpated and examined microscopically. Intramammary inoculation and subcutaneous injection with simultaneous crushing of the lymph glands are followed in five days by the appearance of nodular swellings in the glands concerned. Test inoculations of guinea-pigs is the most certain method of clinically diagnosing tuberculosis. Concerning inoculation with tuberculin, see page 258 .

Fowl Cholera. - A pigeon is inoculated subcutaneously with blood from a dead bird. A drop of blood from the dead bird is introduced under the skin of the breast of the pigeon by means of a lancet to the depth of a millimetre. If cholera is present, the musculature beneath the point of inoculation becomes necrotic 
and yellow and the pigeon dies in 12 to 48 hours; on post-mortem examination a characteristic picture of cholera is found (hemorrhagic enteritis) and the specific cholera bacilli are recovered from the blood.

6. SwINe ERYsipelas.-Subcutaneous inoculation of mice (skin pocket) or pigeons (breast) is followed in 24 hours by characteristic symptoms: dejection, roughened hair, dyspncea, mucous discharge from the eyes and agglutination of the eyelids. Mice die in 2 to 4 days; pigeons in 3 to 4 days; the specific bacilli are found in the blood.

7. Stranglas. - The most suitable inoculation animals are white mice, which are immune to glanders. The inoculation is made subcutaneously in the sacral region with a drop of pus or nasal discharge. Death usually occurs in 3 to 6 days and on post-mortem examination a pronounced picture of septicæmia is presented (enlarged spleen, cloudy swelling of the internal organs, exudate in the body cavities, blood infiltration at the point of inoculation); in the blood, the specific strangles cocci are found. More rarely, the animals die in 10 to 20 days with symptoms of metatastic pyæmia. Field mice react only locally to strangles inoculation, which is contrary to their behavior to glanders inoculation.

8. RABIES.-The best method is the intra-ocular inoculation of rabbits with the brain substance of the suspected dog; the material should be obtained in as fresh condition as possible. After 12 to 14 days, the inoculated animal dies of dumb rabies. Intramuscular and subconjunctival injection are also simple methods, but the subdural (intracranial, lumbar) and intracerebral inoculation of rabbits is more complicated.

9. Hemorrhagic Septiczmia.--Rabbits and mice die in 6 hours after cutaneous and subcutaneous inoculation, and in $\mathbf{1 2}$ to 24 hours after inoculation by feeding; large numbers of the bacilli of hemorrhagic septicæmia are found in the blood; a further characteristic in rabbits is hemorrhagic tracheitis.

10. Malignant CEdema.-Only subcutaneous inoculations are effective with mice and guinea-pigs; cutaneous inoculations are 
without effect (contrary to anthrax). The inoculated animals die in 8 to 14 hours with symptoms of an extensive subcutaneous œdema, in which the characteristic bacilli are easily demonstrated. Guinea-pigs are also inoculated subcutaneously for black leg.

Tuberculin Injection.- Tuberculin is obtained by the evaporation of bouillon cultures of tubercle bacilli and is official [in Germany] in two forms: as fluid (old tuberculin) and as dried tuberculin (dry tuberculin, tuberculol). [In the United States, the evaporated tuberculin is diluted with a $1 / 2$ per cent. solution of carbolic acid before it is sent out to the practitioner, the extent of the dilution varying in different laboratories.] Tuberculous animals are very sensitive to tuberculin and react to it with fever and local inflammation. This reaction indicates the presence of tuberculosis; not with certainty, it is true, but yet with great probability. Therefore, tuberculin is, after all, a very valuable diagnostic agent. It is employed according to different methods:

1. Subcutaneous (thermal reaction).

2. Conjunctival (ophthalmic reaction, eye test).

3. Cutaneous and intracutaneous (dermal reaction).

1. The Subcutaneous Tuberculin Test (thermal reaction) is the oldest method. Fluid tuberculin diluted [in Germany] with ten times the amount of $1 / 2$ per cent. carbolic acid solution is injected under the skin. The dose of the tuberculin for cows is $0.5 \mathrm{gram}$, for calves $0.1 \mathrm{gram}$. [The dose of the preparations of tuberculin used in the United States varies from 1 c.c. to 4 c.c., depending upon the extent to which the evaporated tuberculin has been diluted with the $1 / 2$ per cent. carbolic acid solution.] The diagnostic reaction consists of a rise of temperature of at least $2^{\circ} \mathrm{F}$., which occurs 12 to 15 hours after the injection. [It is very generally agreed that a reaction may occur or begin at any time from the 9th to the 20th hour after injection, and it is therefore considered necessary to begin the temperature measurements not later than the 9th hour and to continue them in all cases until at least the 20th hour after the tuberculin is injected. In any case in which the temperature is rising at the 20th hour, 
the temperature measurements should be continued until the temperature begins to fall or until a reaction occurs. American veterinary sanitary authorities recommend that at least three temperature measurements be taken before the tuberculin is injected, at intervals of 2 or 3 hours, and that the tuberculin be injected preferably between 6 and 9 P.M.] The disadvantages of the subcutaneous method are that it is misleading in 13 per cent. of the cases tested (incorrect diagnosis); that it requires a great amount of time, as at least two temperature measurements must be taken before and four after the injection; that it cannot be applied to animals in a febrile condition; that cattle previously injected with tuberculin will not react to the usual dose but require five times the quantity; that the general condition and the milk secretion is influenced unfavorably by the reaction fever, and that now and then chronic tuberculosis is transformed into the acute form. [According to American statistics, errors in diagnosis are much fewer than are indicated by the German figures given above. Statistics compiled by the Bureau of Animal Industry $^{2}$ show that of 24,784 cattle which reacted to tuberculin from 1893 to $1908,24,387$ were found to be tuberculous on postmortem, which is only 1.07 per cent. failures.]

2. The Ophthalmic Test (ophthalmic reaction, conjunctival reaction) has been recently given the preference [in Germany] over the subcutaneous method on account of its greater certainty, simplicity, cheapness, and harmlessness, and also because it is not influenced by the presence of fever or by a previous subcutaneous injection. It is applied in the following manner: four drops of a 5 per cent. solution of dry tuberculin or of a 25 per cent. solution of tuberculol are introduced into the conjunctival sac, or a quantity of a 1 to 2 per cent. tuberculin vaseline the size of a pea is placed in the conjunctival sac with a glass rod. A typical purulent discharge from the eye and a pronounced cedema appear in 12 to 14 hours. Rectal, vaginal, and nasal applications have the same effect. [In Pennsylvania, an alcoholic precipitate of a glycerin

2 Twenty-fifth Annual Report, Bureau of Animal Industry, p. 99. 
bouillon culture of tubercle bacilli suspended in physiological salt solution is used. Two suspensions are made, one containing 4 per cent. of the precipitate and the other 8 per cent. Two drops of the 4 per cent. suspension are placed in the eye to sensitize it, and 4 days later two drops of the 8 per cent. suspension are placed in the same eye. The reaction occurs in 6 to 14 hours. As yet, however, the test has not been substituted for the subcutaneous test.]

3. The Cutantous and Intracotaneous method consists in the application of a concentrated tuberculin solution to the shaved or scarified skin, or its injection into the tissue of the cutis. The dermal reaction is a pronounced cedematous, puffy swelling at the place of inoculation (lateral surface of the neck, caudalanal fold).

In order to insure the greatest possible certainty in diagnosis, it is recommended that all three tests, the subcutaneous, conjunctival, and cutaneous, be applied (simultaneous method).

Mallein Injection.-Fluid mallein (raw mallein), obtained by the evaporation of cultures of glanders bacilli, and dry mallein, an alcoholic precipitate of the fluid mallein, are introduced into the animal body in order to utilize for diagnostic purposes the excessive sensibility of infected animals to mallein, manifested in the form of local symptoms of inflammation or symptoms of a general febrile affection. According to the place of application of the mallein (conjunctiva, subcutis, cutis), three methods of malleinization are distinguished:

(a) The ophthalmic test or ophthalmic reaction.

(b) The subcutaneous test or thermal reaction.

(c) The cutaneous test or cuti-reaction.

(a) The Ophthalmic Test (ophthalmic reaction, conjunctival malleinization) is applied by introducing into the conjunctival sac with a brush or dropper a few drops of fluid mallein ( 0.2 c.c. or 0.2 gram per horse, 0.5 c.c. or 0.5 gram for 5 horses), or a few drops of a 1 per cent. solution of dry mallein [precipitated mallein]. The other eye serves as a control. The specific reaction usually begins 5 to 6 hours after the instillation and continues 36 to 48 
hours. It consists of a purulent discharge from the eye, congestion and swelling of the conjunctiva, and swelling and agglutination of the eyelids. The result of the test is to be determined at the twelfth hour after the instillation at the earliest, and at the twentyfourth hour at the latest. A positive reaction is a certain indication of the presence of glanders. On the other hand, the presence of glanders is not positively excluded by a single negative reaction, but only when a second test three weeks after the first also gives a negative reaction. In the early stages of glanders the hypersensitiveness to mallein is absent; it does not appear until toward the second week after the infection. In doubtful cases (serous or mucous discharge from the eye), the eye test sometimes gives a positive result when repeated on the same day (sensitization). With a positive reaction, an increase of temperature to over $38.5^{\circ} \mathrm{C}$. [101.3 ${ }^{\circ} \mathrm{F}$.] usually occurs in $\mathbf{2 4}$ hours which is also of diagnostic importance. The presence of fever is not a contraindication to the application of the eye test. (To be preferred to the subcutaneous method.)

In recent years, the eye test has been found to be the most trustworthy, the simplest, the most convenient, the cheapest, the most rapid and, for examining large numbers of horses (remounts, imported horses), the most suitable method of diagnosing glanders, especially in Austria, where it has been recognized by the veterinary sanitary laws since 1910 (civil and military laws), in Denmark and in Germany (Prussia, Würtemburg). Recently, it has been officially prescribed in Bavaria.

(b) The Subcutaneous Method or the thermal reaction consists [in Germany] in the subcutaneous injection of 0.02 gram of dry mallein or 0.5 gram of raw mallein. [In the United States, the evaporated glycerin bouillon culture of bacillus mallei is diluted in the laboratory with $1 / 2$ per cent. carbolic acid solution, and the quantity injected depends upon the extent of the dilution.] A rise of temperature of over $2^{\circ} \mathrm{C}$. [3.6 $6^{\circ} \mathrm{F}$.] during the two days following the injection, with a typical temperature curve with two apexes, is to be regarded as a positive indication of glanders, while horses which do not show any febrile reaction and those with 
a temperature rise of less than $1.2^{\circ} \mathrm{C} .\left[2.1^{\circ} \mathrm{F}\right.$.] are to be considered free from the disease. The reports from different countries concerning the diagnostic value of subcutaneous malleinization are contradictory. [In the United States, the Bureau of Animal Industry and the Pennsylvania Livestock Sanitary Board have abandoned it for the ophthalmic test.] A disadvantage of the method is that it cannot be applied to horses in a febrile condition.

[The directions for applying the subcutaneous mallein test, as published by the U. S. Bureau of Animal Industry in 1910 and 1912, are as follows:

The preferable site for injection is on the side of the neck about the centre, where any local swelling is plainly visible. The hair should be clipped from an area about 2 inches in diameter, and the skin thoroughly cleansed with a disinfecting solution, such as $\mathbf{5}$ per cent. carbolic acid. Carefully sterilize the syringe and needle before commencing the injection of each group of animals, and immerse the needle in a disinfecting solution before injecting each animal. It is better to use a separate syringe, needle, and thermometer for animals exhibiting symptoms suspicious of glanders. Carbolized oil, vaseline, or lard should be used to facilitate the insertion of thermometers and also to disinfect them. On the day of injection, the temperature of each animal should be recorded not less than three times at intervals of not less than two hours; for instance, at 2,5 , and 8 P.M. A careful clinical examination of each animal. should also be made, and to each one some designation should be given by which the animal will be known throughout the test. Mallein may then be injected at 8 or 10 P.M., providing the preliminary temperatures are not abnormal. After injection the temperatures should again be recorded, starting at the expiration of not more than 10 hours, and should be repeated at intervals of approximately 2 hours until the expiration of at least 20 hours from the time of injection, and should be continued over a longer period in the case of an animal with a rising temperature at the twentieth hour, if, at the same time, a local reaction is present. What constitutes a reaction sufficient to warrant condemnation of the animal has been the subject of many articles and prolonged discussion. The Bureau of Animal Industry has adopted the following uniform principles for judging the mallein test:

1. In order that a reaction produced by mallein may be considered positive it should evince the characteristics of a typical reaction; that is, a combination of thermal, local, and general reactions.

2. By a typical reaction is to be understood a gradual rise of temperature of at least $3^{\circ} \mathrm{F}$. and to above $104^{\circ} \mathrm{F}$., the maximum temperature being sustained in the form of a single or double plateau. It should be accompanied by a local as well as a general reaction. 
The local reaction consists of an infiltration at the site of injection, forming a large, abrupt, painful swelling with radiating lymphatics appearing as raised cords, generally attaining greatest prominence at from 18 to 21 hours after injection. The general reaction is exhibited by a stiffened gait, depression, loss of appetite, and accelerated breathing.

3. The presence of a local reaction, especially when associated with a general reaction, should be regarded as evidence of glanders, even if the thermal reaction be slight or absent.

4. Animals giving an atypical reaction and those reaching a maximum temperature of $103^{\circ} \mathrm{F}$. should be retested after the expiration of not less than 15 days.]

(c) Cutaneous malleinization consists of the injection of mallein into the scarified skin and the pronounced local swelling which is produced. The method is complicated, technically difficult and the reaction varies with the method of application (superficial or deep scarification).

\section{Directions for Applying the Mallein Eye Test.}

I. Nature of the Test.-Horses infected with glanders are hypersensitive to mallein. They therefore react when mallein is instilled into the conjunctival sac with specific local symptoms of inflammation. The specific reaction is a pronounced redness and swelling and a purulent inflammation of the conjunctiva of the eyelids, especially in the inner canthus of the eye (drops of pus, flakes of pus in the discharge, purulent discharge). A serous, seromucous or mucous discharge is not specific. The specific reaction does not begin immediately, but at the earliest 5 to 6 hours after the instillation of the mallein; it usually continues for 36 to 48 hours, sometimes longer. The best time to observe it is from the twelfth to the twenty-fourth hour. The slight symptoms of irritation of the conjunctiva (tears, photophobia, slight redness of the conjunctiva), which occur in many horses very soon after the mallein is instilled and disappear after a few hours, must not be mistaken for the specific mallein reaction.

II. Application.-Either fluid mallein (raw mallein) or dry [precipitated] mallein is used.

With a brush or dropper (eye pipette), a few drops of fluid, undiluted mallein or of a freshly-prepared 1 per cent. solution of dry [precipitated] mallein in distilled water or physiological salt solution are introduced into the right eye. The left eye serves as a control.

In order that the eye may be examined for the symptoms of the specific reaction in daylight, it is recommended that the test be begun in the morning 
or in the evening. When the mallein is instilled in the morning, the reaction will appear at the earliest in the afternoon; when it is made in the evening, the first observation of the eye can be made the next morning.

Since drops of pus flowing from the eye and sticking to the hair may be thrown off by movements of the head and thus escape detection, restless horses should be tied up short during the test.

The presence of fever does not interfere with the application of the test.

The test does not influence the blood test.

III. Judgment.-The test may terminate in three ways: positive, negative, doubtful.

The reaction is positive if a purulent discharge from the eye occurs in 12 to 24 hours.

The reaction is negative if a discharge from the eye does not appear in 12 to 24 hours.

The reaction is doubtful if after 12 to 24 hours only a serous, seromucous or mucous discharge occurs from the eye, or if only a drop of purulent secretion collects in the inner canthus of the eye without any discharge.

The judgment of the test is based upon the following principles:

1. The presence of glanders is assumed as probable if a positive reaction occurs 12 to 24 hours after the instillation of the mallein.

2. The absence of glanders is assumed as probable if a negative reaction occurs 12 to 24 hours after the instillation of the mallein and if, in addition, a negative reaction is again obtained upon a repetition of the test three weeks later. (In the initial stages of glanders the hypersensitiveness to mallein is absent; this appears only toward the second week after infection.)

3. If the reaction to the first test is doubtful, a second test is begun on the same day. If the reaction to the second test is positive, the presence of glanders is assumed as probable. If the reaction to the second test is negative or doubtful, then a third test is made after 3 weeks. If the reaction to the third test is negative, it is assumed as probable that glanders is not present. If the reaction to the third test is positive, the presence of glanders is assumed as probable. If the reaction to the third test is doubtful, the horse remains under suspicion of glanders. 


\section{WATER AS A REMEDY. HYDROTHERAPY}

Synonyms: Hydrotherapy, hydriatrics, balneotherapy, Priessnitz's' cure, water-cure.

General.-Water has been employed as a healing remedy since ancient times. In spite of this, it is not possible to present to-day a clear, scientific analysis of its method of action. Some of the undoubted successes of hydrotherapy are still based upon pure empiricism (Priessnitz), and cannot at this time be scientifically explained at all, or only incompletely. The difficulty of placing hydrotherapy on a rational foundation is due to the circumstance that the effect of water upon the body is very complicated. Several very different factors apparently coöperate in the water-cure. Not only the water itself, but also its temperature and likewise certain mechanical factors appear to exert a combined action. The continuance of the application and the change from cold to warm water are also of importance. Among the different properties of water, the irritant action of cold water upon the skin is of the greatest consideration in veterinary therapeutics. The internal use of water (mineral water), as ordinarily employed in human medicine, is not practicable in veterinary medicine. As with the other cutaneous irritants, the effect of water upon the body is very extensive, which accounts for the frequent use of hydrotherapy in the most varied disease conditions.

Actions.-1. Upon THE SKIN water has in the first place a cleansing effect, and consequently in a certain sense a disinfectant action. In addition, when its influence is long continued, it brings about a swelling and loosening (maceration) of the epithelial cells, with increased desquamation of the same. Since the capacity of cold water for heat is very great, it takes up heat from the body

1 Vincent Priessnitz, agriculturist in Gräfenberg, Austrian Silesia; lived from 1799 to 1851 , and founded in 1826 the first hydropathic establishment. 
when applied locally or generally and therefore operates as an antipyretic in conditions of abnormally high temperature. Cold and hot water act as cutaneous irritants, producing at first a contraction of the cutaneous vessels and of the smooth muscle of the skin with a consequent anæmia, followed by a pronounced reactive relaxation of the vessels of the skin and of the underlying parts with a decided hyperæmia of the same. On this account, cold water is to be regarded as an epispastic rubefacient which not only influences and changes the local circulatory relations of the skin and the tissues beneath it (subcutis, tendons, tendon sheaths, muscles, articulations, bones) but also, when used extensively, affects the blood distribution of the entire body, causing the blood to flow from the centre of the body to the periphery and depleting the internal organs. While cold water produces a passive hyperæmia, hot water, and moderately warm water when the application is long continued (cataplasms), generate an active hyperæmia. These actions upon the skin point to numerous indications for the treatment of local diseases of the skin, the subcutis, the musculature, tendons, bones and articulations as well as general febrile and internal diseases. Furthermore, the hyperæmia produced considerably stimulates the secretory activity of the skin (water, urea and metabolic products), sometimes even to the extent of producing perspiration. Since a relation exists between the secretory functions of the skin and different internal organs, especially the kidneys and lungs, a therapeutic action may be exerted upon these organs by the use of water. Depending upon the temperature, water exerts a stimulant action upon nerves or exerts a sedative effect in abnormal, painful conditions.

Of great practical importance in all these cases, in addition to the temperature of the water, is the continuance of its action and a change from cold to warm. In reference to the temperature, a distinction must be made between cold $\left(0-15^{\circ} \mathrm{C}\right.$.), tepid (15$30^{\circ}$ C. ), warm $\left(30-38^{\circ}\right.$ C. $)$ and hot $\left(38^{\circ} \mathrm{C}\right.$. and above). Only cold and hot water exert a pronounced action as cutaneous irritants in the manner described above. Warm water corresponding in 
temperature to that of the body is indifferent, and the action of tepid water is weak. The colder or the warmer the water in comparison to the body temperature of the animal, the more pronounced is its action upon the skin. In regard to the continuance of action, when cold water is used for the purpose of reducing temperature its application must naturally be continued as long as possible. But if the water is employed as a cutaneous irritant, then the therapeutic effect is in direct relation to the shortness and frequency of the individual applications. When a single application of cold water produces a reaction in the skin in the form of a change in the circulatory relations (hyperæmia), the condition disappears after a certain interval, and a renewal of the cutaneous irritation is naturally required to again bring about the same reaction with its healing effects upon the organism. One longcontinued application of cold water produces only a single reaction, which occurs in the beginning. For these reasons frequent applications are indicated, and on this account Priessnitz dressings, for example, are changed frequently (on the average every 3 hours). The effects produced under these circumstances are as follows: When the dressing which has been dipped in cold water is first applied, anæmia and cooling of the skin occurs; this is gradually followed by a hyperæmia, which is continued several hours and which is promoted by the warm overlying dressing. After this period, the circulatory relations are again equalized and in order to produce a new reaction the cold irritant must be renewed; i.e., a new cold dressing must be applied.

Failure of a patient to show any reaction in the skin after a proper application of cold water is evidence of great weakness of the nervous system and justifies an unfavorable prognosis. For prophylactic purposes, the frequent use of cold water in healthy animals is recommended to facilitate the occurrence of the reaction and to exercise and strengthen the skin (cold rub-down for horses and cattle, washing).

2. The circolatory apparatus is influenced in very different directions by hydrotherapy. As already observed, cold water 
causes at first, in consequence of irritation of the peripheral vasomotor centres (Lenaschew, Plügers Archiv., vol. 26), a contraction of the cutaneous vessels with anæmia of the skin, which drives the blood to the interior and also increases the blood-pressure, the activity of the heart and the internal temperature. With the occurrence of the reaction in the skin, contrary effects are produced. The blood-vessels dilate, the blood flows from the centre to the periphery, and blood-pressure, heart activity, pulse frequency and internal temperature decrease. It is therefore possible to act with hydrotherapy upon every single factor of the circulation: upon the lumen and tension of the blood-vessels, upon the heart, upon the blood-pressure, upon the blood distribution and upon the blood heat. Water is consequently a valuable remedy in all conditions of inflammation and congestion and in general febrile diseases. It is also a derivative remedy, particularly on account of its regulating influence upon blood distribution. Interesting investigations have been made by Schüller (Deutsches Archiv. für Klin. Medizin, vol. 14) concerning the derivative effect of water upon the deeply situated organs, which are of therapeutic importance, especially in inflammation of the brain. In trephined rabbits, constriction of the vessels of the pia and contraction of the brain were observed after the application of warm compresses to the skin, while the employment of cold dressings on the skin or a cold bath caused a dilation of the vessels of the pia. The same action apparently extends to the spinal cord, the lungs and the other viscera. These experimentally established facts justify scientifically the hydropathic derivation which has been hitherto practised empirically, especially in inflammation of the brain, pneumonia, pleuritis and peritonitis. Finally, since cold water also increases metabolism, as is shown by the increased elimination of carbon dioxide and the increased absorption of oxygen demonstrated in animals experimentally by Röhrig and Zuntz, the resorbent effects of external applications of water in connection with the stimulation of the circulation are readily understood.

3. The Nervous system is affected in different ways, accord- 
ing to the temperature of the water. Cold water stimulates the activity of nerve tissue; warm water, on the contrary, has a sedative effect, soothing and depressing. Depending upon whether the application of the water is local or general, a local or total alteration of innervation can be brought about, either of the nature of a stimulation or depression, which is not possible with other cutaneousirritants. Upon the nerves of the skin the action is direct, while the other parts of the nervous system are influenced reflexly through the skin (counter-irritation). By the application of cold water, a weakened nervous system is stimulated, entirely independent of the change in circulation (derivation), while warm water applications depress an abnormally stimulated nervous apparatus. This is true of the brain and spinal cord as well as of the nerves of the different internal organs, such as the stomach, intestines, kidneys, uterus, lungs, heart, etc. Well-known examples of these actions of water are the stimulant effects of cold and the sedative, anodyne effects of warm water applications in colic of the horse and the favorable action of the Priessnitz dressing in abnormal sensibility of the larynx (cough), pharynx (difficult swallowing), brain, musculature (rheumatism), articulations and tendons (inflammation).

4. The BoDy CeLLS are nutritively stimulated by moist heat (cold acts reversely) and their function of forming antitoxins and their regeneration activity are thereby accelerated. Local leucocytosis is also promoted by heat, but is inhibited by cold (see the chapter on acrics).

5. The Glands of the body (liver, kidneys, pancreas; gastric, intestinal and cutaneous glands) are also influenced in different ways by applications of water, partly through the circulation (derivation) and partly through the nervous system (counter-irritation). The secretions of the liver and kidneys in particular are stimulated by cold and decreased by warm water applications.

6. The Musculature has, as is well known, special relations with the skin; the diseases caused by chilling (rheumatism, rheumatic hæmoglobinæmia [azoturia]) demonstrate that the two are 
intimately associated. In these diseases, it may be assumed, the chilling acts as an abnormal cutaneous irritant, causing a disturbance of the circulation, innervation and especially the metabolism of the musculature. Similar influences can be exerted upon the diseased musculature by the employment of hydropathic dressings, the disturbed circulation and innervation being affected by derivation and counter-irritation. Warm water is most desirable for this purpose because it at the same time allayspain. Cold water is more suitable to the prophylactic hardening against the diseases mentioned. Experience has taught that muscular rheumatism as well as rheumatic hæmoglobinæmia [azoturia] can be effectively prevented by accustoming the skin to the irritation from cold and hardening it against the harmful results. Avoiding too warm stabling and covering, accustoming to cold, and especially frequent cold rubbing down when rationally employed afford good protection against these diseases.

7. The respiration is stimulated by cold water. Cold applications are therefore employed as stimulants in weakness and paralytic conditions of the respiratory centre (syncope, poisoning by chloroform) and to strengthen expiration in pulmonary and bronchial diseases. On the other hand, the diseased lungs are relieved by the derivation of the blood from the lungs to the skin (cutaneous respiration) by means of moist, warm applications. In these respects hydrotherapy is of importance in the treatment of pneumonia and other respiratory diseases.

8. LOCALLY, cold at first causes contraction of the arteries and anæmia (checking hemorrhage, antiphlogistic action), later dilation and congestion of the veins with slowing of the circulation and resorption and decrease of the leucocytes. Moist heat, especially the Priessnitz dressing and cataplasms, promotes local blood circulation and cell activity and also local leucocytosis and the formation of antitoxins. Hot applications generate an active hyperæmia, which extends to a considerable depth (musculature, peritoneum) and continues for 24 hours and longer; in addition, the circulation of lymph and consequently resorption is stimulated 
(hyperlymphia, cedema formation). See the experimental investigations of Schäffer. ${ }^{2}$

Hyperæmia as a Healing Remedy.-Bier ${ }^{3}$ has drawn attention to the favorable effects of chronic passive hyperæmia, produced artificially by elastic bandages and by hot-air apparatuses, upon surgical suppurative and inflammatory processes. Passive hyperæmia, like active hyperæmia, has a resorbent, bactericidal and anodyne action and stimulates the formation of new tissue. The same effects are produced by the continued employment of high degrees of heat by means of Ulimann's $\mathrm{s}^{4}$ hydro-thermo regulator, which was introduced into veterinary surgery by Bayer ${ }^{5}$ and Eberlein ${ }^{6}$ and found by them to be of value especially in chronic tendinitis, tendovaginitis, shoulder boils, exostoses, thickening of the skin, articular and muscular diseases, wounds and ulcers of the horse (constant, regulated heat treatment in the form of dry or moist heat). Upon the basis of his experiences in the Vienna clinic, Schmidt ${ }^{7}$ has formed the following conclusions concerning the value of Bier's passive hyperæmia in veterinary medicine: The aspirator is most useful in diseases of the paws of small animals (phlegmon, panaritium). The rubber tubing and the elastic bandage can be used on all of the domestic animals, but the technique presents numerous difficulties, which are greater in animals than in man. Purulent inflammations of the articulations and tendon sheaths and phlegmonous processes in the hoof are best suited to the passive hyperæmic treatment. On account of the technical difficulties and the dangers attending the treatment, however, it can only be employed in stationary clinics under continual control; its use in general practice and in polyclinics is precluded. According to Rōder ${ }^{8}$ also, the field for the use of Bier's passive hyperæmia is limited; he recommends venous stasis by means of bandages in wounds of the coronet, in contused wounds and after resection of the lateral cartilage, and the employment of the aspirator in shoulder boils. Krüger," as a result

${ }^{2}$ Schäffer, Der Einfluss unserer therapeutischen Massaahmen auf die Entzündung. Stuttgart, 1907, Ferd. Enke.

s Bier, Die Hyperämie als Heilnittel. Leipzig, 5. Aufl., 1907.

4 Ulmann, Wirkungen und therapeutische Verwertung konstanter Wärmeapplikationen. Physiklinisch-medizinische Monatshefte, 1904.

' Bayer, Der Hydrothermoregulator. Zeitschr. f. Tiermed., 1903.

- Eberlein, Der Hydrothermoregulator. Berl. Archiv., 1905.

'Schmidt, Die Biersche Stauungshyperämie in der Tierheilkunde. Monatshefte für prakt. Tierheilkde., 1907.

s Röder, Dresdener Naturforscherversammlung, 1907.

- Krūger, Hyperämie als Heilmittel in der Tierheilkunde. Zeitschr. für Vet., 1910. 
of his experiences in the Berlin Horse-shoeing School, agrees with Schmidt and Röder that passive hyperæmia, when properly employed in suitable cases, is to be regarded as a good healing method in veterinary medicine. Eberlein and Braun $^{10}$ have cured four cases of inflammation of the temporomaxillary articulation in the horse with Klapp's aspirator.

Uses.-The external use of water for healing purposes is in many cases similar to the use of the cutaneous irritants (see p. 148). The indications for the employment of hydrotherapy are, however, more numerous than for the use of cutaneous irritants. Moreover, the water is often preferred to these drugs because of the simplicity of its application and the cheapness of the hydropathic apparatuses. The most important diseased conditions treated with water are the following:

1. Local surgical affections of the skin, subcutis, tendons, tendon sheaths, muscles, joints, bones, and lymph glands. The application of cold water is indicated only in active hyperæmia and in entirely fresh, acute inflammatory conditions in these parts. On the other hand, warm, moist applications or cataplasms are indicated in all subacute and chronic inflammations, in passive hyperæmia and in extravasations in the parts named. This is true especially of fluid and solid exudates, indurations, old swellings and thickenings of the skin, subcutis, tendons, articulations, bones and muscles, and also of purulent inflammations and ripening abscesses. The moist heat in these cases dilates the vessels, accelerates the disturbed and sluggish blood and lymph circulation, promotes metabolism and resorption, softens hard inflammatory products, encourages the outwandering of white blood-cells (phagocytosis, histolysis), accelerates connective tissue formation and cicatrization and stimulates the formation of antitoxins.

2. General febrile diseases. The antipyretic action of cold water in the form of cold poultices, irrigations, douches, baths, fomentations and rectal infusions depends upon the withdrawal of heat; the cold must continue to operate for a long time, however,

${ }^{10}$ Braun, Die Saugbehandlung nach Klapp bei der Arthritis purulenta des Kiefergelenks des Pferdes. Monatshefte für prakt. Tierheilkunde, 1912. 
because a temporary application causes at first a rise of the internal temperature. Warm, moist applications also exert an antipyretic effect, since under their influence blood is drawn from the centre of the body to the periphery and gives off heat; coöperating with this action is the influence upon the vasomotor and caloric nerve centres.

3. Inflammation of the lungs, pleura, peritoneum, brain and spinal cord, stomach and intestines, kidneys, liver, and uterus. The hydropathic effect of warm, moist applications consists of the contraction of the dilated vessels in diseased internal organs and of the derivation of the blood to the skin.

4. Edema of the lungs, brain, and glottis. The action is the same.

5. Muscular rheumatism. The warm, moist applications exert a derivative and anodyne action.

6. Colic and cough. The action is the same.

7. Paralytic conditions of the nervous system (cerebral, spinal and peripheral paralyses, weakness of the loins, parturient paresis, poisonings, paresis of the gastric and intestinal musculature). Cold or hot water (cutaneous irritation, counter-irritation) is indicated in these conditions.

8. Excited conditions of the nervous system (pains, spasms, hyperæsthesia, increased reflex activity). Warm water (sedative action) is employed in these conditions.

9. Exudates (fluid and solid) in the internal organs. The warm, moist applications promote the circulation, leucocytosis, metabolism, and resorption.

10. Kidney and liver diseases (stimulation of the secretion, derivation).

11. Catarrhs of the respiratory apparatus (inhalation of steam).

12. Obesity (stimulation of metabolism).

Forms of Application.-1. Priessnitz's dressing. This is applied in the following manner: The skin is first covered with a piece of linen or cotton cloth, or a bandage, which has been dipped in cold water, and over this cold, wet dressing a dry, woollen cover- 
ing or bandage is so applied that the surface of the dressing remains entirely dry (warm, moist poultice). After about 1 to 3 hours, a reactive hyperæmia of the skin has taken place under the bandage in the manner before described, so that the dressing must be renewed after this period. A renewal of the dressing is also indicated when the inner layers of the bandage have become dry. On account of the early occurrence of maceration of the skin, care must be taken not to continue the use of the dressing too long; in order to avoid inflammation of the skin, it is recommended that the dressing be left off over night or every second or third day.

2. Irrigation. By this is understood the irrigation of the body or parts of it with cold water by means of a rubber hose. This serves to withdraw heat generally and locally in fever and in inflammations of peripheral parts (hoofs, tendons, joints, head).

3. Douche. This is the application of a stream of cold water with the simultaneous action of mechanical force (pressure from a syringe or from water pipes, irrigation from a higher level); it acts as a powerful stimulant to the nervous system, especially in depressed conditions of the brain and spinal cord.

4. Baths. The domestic animals are in general rarely subjected to complete baths for hydrotherapeutic purposes; on the other hand, cold and warm local baths are employed in different forms, especially foot baths for horses, cattle and dogs, for the purpose of reducing temperature, softening and cleansing. According to Wenz (Dissertation, Giessen, 1911), a preliminary warm bath increases the effect of a succeeding cold bath, while the reverse of this order is unfavorable. Additional investigations on the action and use of baths on animals have been published by Lucas (Berl. Arch., 1910).

5. Cold rectal infusions. These serve to reduce the temperature in fever and to stimulate intestinal peristalsis and evacuate the rectum; they are used most frequently on the horse (clysters, irrigations).

6. Clay poultices. This is the oldest form of hydrotherapy used in veterinary medicine. The cooling effect is slight, accord- 
ing to Bayer, the temperature being only temporarily depressed a few degrees.

7. Cataplasms. These are effective especially because of their heat and moisture.

Other forms of hydrotherapy which may be mentioned are simple cold and hot poultices, the ice poultice (ice pack), Leiter's cooling apparatus, and the different cold and warm washes. In regard to the use of the thermo-regulator, see the text-books on operations and instruments. This apparatus makes possible the uninterrupted (24 hours and more) action of radiating heat of 42 to $44^{\circ} \mathrm{C}$. 


\section{MASSAGE}

Synongm: Mechanotherapy.

Nature and Forms.-Massage is the application of pressure upon the skin and the parts beneath it. It has been used for a long time in veterinary medicine, intentionally in the form of rubbing and unintentionally in the application of ointments. It is also one of the oldest methods of healing used in the treatment of man (Chinese, Greeks, Romans, primitive people). After being forgotten for a long time (during the entire middle ages up to modern times), the method was to a certain extent rediscovered in Sweden by Per Hendrick Ling (Swedish medical gymnastics). Zander displaced the masseur by mechanical apparatus (mechanotherapy). Mezger, Mosengeil, Zabludowski and others have recently built up the method practically and scientifically. The following varieties of massage are distinguished:

1. Stroking (EFfleurage).-This consists in passing the finger tips or the flat of the hand superficially and gently over the skin.

2. Rubbing (Massage À Friction).-In this variety of massage, the skin is rubbed under strong pressure.

3. Kneading (PÉtrissage).- - The part of the body concerned is pressed with the finger tips or fist as dough is kneaded.

4. Tapping (Tapotgment).-The diseased member is tapped or struck at short intervals (edge of the hand, fist, stick). Tapping is employed in paralyses of muscles and nerves and as preliminary massage in the neighborhood of articulations.

In addition, compression (constant pressure), vibration (intermittent pressure by special apparatuses) and active and passive movement (mechanotherapy in the restricted sense, Swedish medical gymnastics) may be considered as forms of massage.

Action.-As in the case of cutaneous irritation and hydrotherapy, the effect of massage upon the body is very extensive. 276 
The action appears to be not exclusively mechanical but partly dynamic. The most important local effects of massage are a cleansing, stimulant, anodyne and a dynamic action. In addition, massage produces important general effects. Its influence upon the individual organs is as follows:

1. The circulation of the blood and lymph is influenced by massage first of all. The action here is the same as that which occurs during motion in the vessels of the extremities, where the venous blood and lymph is forced centripetally toward the heart by the contraction of the muscles and the tension of the fascia with the coöperation of the valves of the vessels, a process which may be called a natural or physiological massage. The pressure exerted upon the body by artificial massage produces at first an anæmia of the parts concerned in consequence of the blood and lymph being pressed out of the veins and lymph vessels toward the heart. With the cessation of the pressure, a large amount of fresh blood flows into the empty spaces and the area becomes hyperæmic (aspiratory and pressure action). The repeated alternate occurrence of anæmia and hyperæmia stimulates and accelerates the circulation in the massaged parts.

2. The acceleration of the circulation brings about an increase of metabolism, leucocytosis, histolysis, and local antitoxin formation, promotes retrogressive changes and regeneration, stimulates the resorption of the products of fatigue and metabolism, pathological products, exudates and extravasations, and improves the nutrition of the part massaged (experiments of Mosengeil with pigments injected into the joints).

3. Solid, fibrinous exudates and blood coagula beneath the skin, in tendon sheaths and in joint cavities are mechanically crushed by massage and thus prepared for resorption. In addition, swellings of the skin and mucous membranes, subcutis, musculature, tendons and tendon sheaths, joints, etc., are reduced in volume.

4. Contractions of the musculature are overcome by tapping the muscle; simple rubbing and stroking brings about hyperæmia 
with increased nutrition and blood formation; in addition, the capacity for work is increased, as by training.

5. The nervous system is stimulated by massage in the same manner as by cutaneous irritation. The cutaneous nerves are stimulated by kneading and tapping and the stimulation acts reflexly upon the central nervous system. Paralyzed peripheral nerves (motor and sensory) especially are treated by massage. Light massage (stroking) appears to reduce increased susceptibility of nerves.

6. Other general effects of massage include increase of cardiac activity, pulse and body temperature, reflex stimulation of gastric and intestinal peristalsis, diuresis and diaphoresis, change in the distribution of the blood (depletion of the central organs, derivative action), improvement of the general state of nutrition and strengthening of the body constitution.

Uses.-Massage is used principally in surgery; it can, however, be employed with advantage in internal medicine. But its value in veterinary medicine must not be overestimated. The alleged results of massage in practice are in great part to be attributed to the healing power of nature and to the simultaneous employment of hydrotherapy and medical treatment. For horses especially, massage will never attain the same importance as for man, where suggestion is frequently the principal healing factor; in addition, the hair of the horse, the resistance of many animals and the bodily difficulties arising for the masseur hinder the application of the method. Furthermore, much time is often lost with massage; blisters and firing are much more valuable therapeutic_measures than massage.

The most important indications for massage are the following:

1. Blood stasis, lymph stasis, œdemas of the skin, mucous membranes, and subcutis (especially prophylactically against formation of thrombi and in decubitus).

2. Contusions, hemorrhages and lymphorrhagia on the surface of the body (saddle pressure, hæmatomas of the thigh).

3. Subacute, aseptic inflammations of the skin, subcutis, ten- 
dons, tendon sheaths, joints, bones, glands and udder (milking). Massage should not be applied too early in fresh distortions of the joints and in acute periostitis (only after several days' treatment).

4. Chronic inflammatory thickenings and indurations in the organs mentioned in paragraph 3 , splints, galls, ulcers, badly granulating wounds, stiffness and weakness of the limbs in old horses and after hard work.

5. Muscular rheumatism, muscular paralysis, muscular atrophy and muscular cramp.

6. Paralysis of peripheral, motor and sensory nerves, spinal and cerebral paralytic conditions.

7. Chronic inflammation of the cornea (leucoma); œdema of the eyelids.

8. Colic, constipation, tympanites, paresis of the rumen, atony of the gastric and intestinal musculature.

9. Febrile general diseases, inflammation of internal organs, obesity, diabetes, anæmia (Weir Mitchell's method), cardiac failure (Oertel's method).

10. In healthy animals, massage in the form of training and rubbing down after exercise is an important factor in maintaining bealth, especially in horses.

Contraindications.-Since massage promotes the resorption of the products of inflammation (fibrin, serum, blood), it can be employed only in simple, non-septic inflammations. In all cases in which septic material is present in the area of inflammation, especially the bacteria of septicæmia and pyæmia, massage must be omitted, otherwise a general infection of the body will result from the resorption of the infectious material. Massage is especially contraindicated in phlegmona, abscesses, septic mastitis, metastatic tendovaginitis (contagious pneumonia of horses), erysipelatous swellings (influenza), malignant œdema, black leg, all purulent, sanious and septic inflammatory conditions in general and in fresh keratitis.

Massage is to be avoided particularly in phlebitis, lymphangitis and purulent lymphadenitis, because here the danger of a 
blood infection is the greatest. In addition, phlebotomy fistulæ and venous thrombi should not be massaged, since lung emboli may result from the disintegration of the thrombus. Massage of arterial thrombi cannot be unqualifiedly recommended because of the danger of peripheral emboli. Finally, massage is forbidden in animals in many cases by the local sensitiveness.

Technique of Massage.-To massage correctly requires on the one hand good instruction and practice and on the other hand a certain adaptability of the fingers and hand. Definite rules concerning the variety and the continuance of massage can not be laid down. Massaging according to a fixed system should be especially avoided; each case is to be scientifically individualized. The principle of massage is that the stroking, rubbing and kneading movements should be centripetal where possible, i.e., in the direction from the periphery toward the heart. On the legs, for example, one massages from below upward, and on the neck from above downward, following the course of the venous blood and lymph. The movements may also be circular, from above downward and the reverse alternately; the chief result is then alteration of the circulation. Usually, massage is begun and ended with stroking. The "introductory massage" is begun on the healthy, centrally situated parts in order first of all to clear the paths of diversion. Massage is usually performed with the dry hand (finger tips, surface of the hand, thumbs, fist); when massage is long-continued the hand is moistened or is anointed with pure oil, paraffin ointment, lard or with a medicated ointment (camphor ointment, iodoform ointment). The official paraffin ointment ${ }^{1}$ (white vaseline) is the best. Massage is continued on the average for 10 to 20 minutes and is applied once or twice daily. In massaging articulations, flexing, extending and rotating movements are employed. Very hard indurations, as splints, etc., can be massaged with the assistance of sticks and plates. Aids of this kind are unnecessary in other conditions; this is also true of the use of objects placed upon the skin to receive the pressure or blow. Tapping is used in paralytic conditions and as a preliminary massage in distortions. The surface of the fingers and the hollow of the hand are well adapted for stroking, both hands being used alternately; deep-lying parts are massaged with the tips of the thumbs. Rubbing is performed with the joints of the fingers and hand held rigidly, the hands moving in straight lines or in a circle over small surfaces. In kneading, the soft parts are grasped transversely with the hands and pressed with the fingers progressively toward the centre of the body. Tapping consists of elastic taps with the loose-jointed hand or slapping with the

[1 Petrolatum album of the U. S. Pharmacopcia.] 
hollow of the hand; the fist and instruments are also used. Massage of the rumen and intestines is performed by pressing both fists into the flank region against the organ concerned; general rubbing of the abdominal wall operates indirectly. In colic of the horse, the large colon can be massaged from the rectum. Massage of the cornea is performed by placing some ointment (yellow mercuric oxide salve, $1: 25$ ) between the lids, drawing them over the eye and gently rubbing. The articulations, tendons and muscles are most frequently massaged. For more details of the technique of massage, see the works of von Mosengeil, Reibmayr, Zabludowski, Vogel and others. Concerning the action and uses of massage in veterinary medicine see also Kohlhepp (Dissertation, Giessen, 1906), Goldbeck (Zeitschr. f. Vet., 1908) and Leuffen (Monatshefte für prakt. Tierheilkunde, 1912). 


\section{ELECTRICITY AS A REMEDY. ELECTROTHERAPY}

Synonyms: Galvanotherapy, Faradotherapy, Franklinotherapy.

General.-Although electricity has been employed for healing purposes for a long time, it is only within the last seventy years that its action upon the body has been closely examined scientifically (Remak, Ziemssen, Erb). Furthermore, its physiological action remains unexplained to-day in many respects. Electrotherapy is therefore still in part a purely empirical healing method. In veterinary medicine, electricity is seldom employed for curative purposes and, indeed, is used mostly only on dogs and horses. According to the form of electricity employed and the object of the treatment, the following forms of electrotherapy are differentiated:

1. Galvanotherapy consists in the employment of the continuous (galvanic) current generated in galvanic batteries.

2. Faradotherapy uses the interrupted (faradic, induced) current, which is generated by means of an induction apparatus.

3. FrankLinotherapy is seldom employed; static or friction electricity is used.

4. EleCtRolysis (galvanolysis) is the chemical decomposition of fluids by the galvanic current, the electrodes being placed upon the skin.

5. Elactropuncture (galvanopuncture) serves the same purposes as the needle-shaped electrodes which are used to penetrate the tissues.

6. Galvanocatstic is the use of the galvanic current to heat firing apparatus.

Action.-The effects of electricity upon the living animal body are very complicated and have not been completely investigated. The nervous system and the musculature are influenced first of all by the electric current. But the fluids of the body, especially the blood, and the glands and other tissues appear also 282 
to be changed in certain respects by electricity. In general the following effects are recognized:

1. The stimulant action is produced most strongly by the induced (faradic) current. It causes burning of the skin (cutaneous irritation), stimulation of the sensory and motor nerves, muscular contraction, dilation of the blood-vessels (stimulation of the vasodilators), increase of gland activity and of metabolism. Electricity therefore operates as a stimulant to most of the organs of the body.

2. The electrotonic action consists in the alteration of the physiological electrotonus, i.e., a change in the susceptibility of the nerves (anelectrotonus, catelectrotonus). To this are probably due in great part the curative results of electrotherapy in nerve diseases. The electrotonic action of the continuous current especially is sedative, reducing the receptivity of the nerves.

3. A chemical action is undoubtedly produced by the electric current, especially in the region of the poles (anode, cathode). It acts first of all upon the fluids (serum, blood) and upon the salts contained in them. As is well known, salts and other compounds (water) are separated by electrolysis into their positive and negative elements and the positive (alkaline) elements are attracted by the negative pole or cathode while the negative elements (acid) are attracted by the positive pole or anode. The electric current in muscles and nerves is probably due to a similar combination of chemical opposites.

4. A cataphoric action is manifested by substances which ordinarily are not diffused through the tissues of the body becoming diffusible under the influence of the electric current. Because of the results obtained in experiments with potassium iodide and cocaine, it is assumed that pathological products also become diffusible.

The so-called catalytic (alterative) action appears to consist essentially of a stimulation of the vasomotor and trophic nerves and consequently falls under the first-named action.

Uses.-In veterinary medicine, electrotherapy is indicated in 
the following diseased conditions in the smaller domestic animals and in horses:

1. As a stimulant in pareses and paralyses of the posterior quarters and in paralyses of the peripheral nerves and muscles (paralysis of the facial and radial nerves, tongue, bladder, quadriceps and penis, and amblyopia). In these conditions, the interrupted (faradic, induced) current is used in medium strength, because every single one of the numerous interruptions stimulates the nervous system.

2. As a sedative in excitable conditions of the muscles (twitchings after canine distemper). The galvanic or continuous current is used as mildly as possible.

3. As a diagnostic and prognostic to demonstrate the retention or loss of sensory and motor excitability in paralytic conditions of the nervous system and musculature (testing electric excitability).

The surgical employment of the galvanocaustic and electropuncture for the destruction of new formations has not been made use of in veterinary medicine.

Technique.-The best induction apparatus for veterinary purposes is the small, handy apparatus of Spamer (price, $\$ 7$ to $\$ 10$ ). If the electric treatment is limited to paralytic conditions, this apparatus will be entirely sufficient. For other cases, a small galvanic apparatus can be used. The method of using these apparatuses is described in the directions which accompany them. In general, it is to be remembered that the hairy skin of animals is a poor conductor of electricity and that the electrodes must therefore be moistened (salt water is best) before they are applied. It is also to be remembered that the electric excitability is increased in the region of the negative electrode (cathode), while it is decreased in the region of the anode. Of especial practical importance are the facts already mentioned, namely: that the nerves are soothed by a weak galvanic current, while they are stimulated by a strong faradic current. In the employment of the faradic current, a weak current is used in the beginning and the strength is slowly increased until a reaction (twitching, pain) is obtained; the current is permitted to operate in the latter strength on the paralyzed part for 10 to 15 minutes, one to three times a day. If a weaker current later produces twitching it is an indication of improvement. The strength of current used can be exactly regulated with the apparatus. The application of the electrodes differs; usually one electrode is placed as 
close as possible to the paralyzed nerve or muscle, while the other one is applied in the neighborhood. In spinal paresis of the posterior quarters an electrode is placed on the moistened sole of each hind foot so that the current will pass through the entire posterior parts; in paralysis of a single limb, one electrode is applied to the sole of the foot and the other in the region of the lumbar cord. The spinal cord is either treated longitudinally, with the anode placed upon the upper (anterior) and the cathode upon the lower (posterior) part; or transversely, with the electrodes upon the sternum (anode) and spinal column (cathode); the galvanic current, with the largest electrodes possible, operates best. The brain is treated with the electrodes applied to the back of the neck and to the forehead, the weakest galvanic current possible being used.

A more complete discussion of electrotherapy will be found in Grundriss der Elektrotherapie für Tierärzte by Tereg (Berlin, 1902), and in the special works of Erb, Pierson-Sperling, Rosenthal, Benedikt, Levandowski, Meyer, Gräupner and others.

Thermopenetration.-This term refers to the generation of heat in the inner parts of the body with the assistance of the electric current. The unlimited high-frequency current can be introduced into the body in any strength desired without producing any effect upon the nervous system. It exerts a favorable influence on man, especially. in neuralgias and rbeumatic affections. An electric warm-current healing apparatus called the "Stangerotherm" has been recommended for animals, 


\section{BLEEDING}

Synonyms: Venesection, phlebotomy.

General.-Bleeding, a healing method of the old therapeutics, has in the last several decades very correctly passed out of fashion. A critical examination of its indications has shown that in most cases in which it is employed early it is at least unnecessary, while in a large number of cases it is even directly harmful. This is especially true of its former conventional employment in all febrile and inflammatory diseases. Still, it cannot be entirely dispensed with even to-day, especially in veterinary medicine. There are some very well-defined diseases, especially of horses and cattle, in which bleeding under certain circumstances saves life.

Action.- The most important effect upon the body of the decrease in the total quantity of blood ( 5 to 10 liters in horses and cattle) which results from bleeding is the withdrawal of a large amount of water. In this respect, the effect of bleeding agrees entirely with the action of diaphoretic, sialagogue, diuretic, and laxative drugs, especially arecoline. Following this withdrawal of water, the blood seeks to regain at least its former volume as quickly as possible by taking up any fluids in the body at its disposal (lymph, fluid exudates), creating a temporary hydræmia. Upon this process rests one of the most important effects of bleeding. Another important effect is the derivation of the blood from the internal organs; the quicker the blood is withdrawn in bleeding the more rapidly and promptly this action occurs. The other effects of bleeding upon the body are of no special practical importance. It may be mentioned that bleeding also corrects disturbances of the circulation, removes poisons from the body with the blood and increases proteid metabolism, while the fat and carbohydrate metabolism is depressed (increased excretion of urea, decreased elimination of carbon dioxide and decreased absorption of oxygen, according to Bauer). 
Oses.-Bleeding is still indicated to-day in the following conditions:

1. Laminitis of horses. Experience has taught that a free bleeding as early as possible in this condition, in connection with arecoline, is the best treatment for laminitis (founder).

2. In the first stages of inflammation of the brain, so long as symptoms of cerebral congestion are present (pronounced injection of the visible mucous membranes of the head, increased temperature of the cranium, strong pulsation of the arteries of the head, symptoms of psychic excitement).

3 . In congestion of the lungs with threatened cedema of the lungs.

4. In poisoning of the blood (carbon monoxide, illuminating gas, hæmoglobin, uræmia).

Bleeding is also recommended in rheumatic hæmoglobinæmia [azoturia] of horses, as a prophylactic against parturient apoplexy and in different internal circulatory disturbances (heart diseases). In some sections it is used empirically in the beginning of fattening. On the other hand, its employment has been proven to be not without objection in inflammation of the brain and lungs, in pulmonary hemorrhage and in chlorosis (it is alleged to promote the formation of blood in chlorosis of man). Bleeding exerts no prophylactic effect against infectious diseases, as has been established experimentally by Zschokke.

Transfusion.-This method is unnecessary in veterinary medicine and its use in human medicine is strongly contested. It consists in introducing into the veins of a diseased individual defibrinated blood from an individual of the same species. Transfusion is recommended in excessive loss of blood and in poisoning of the blood (carbon monoxide). It has, however, been recently supplanted by infusion and injections of sodium chloride in solution.

Infusion.-By this is understood the introduction of solutions of medicines directly into the circulation through a vein. In human medicine, infusion of sodium chloride solution especially is recommended in place of transfusion in poisonings, cholera, etc. 


\section{GENERAL THERAPEUTICS OF THE ORGANS OF LOCO- MOTION (MUSCLES, TENDONS, NERVES, ARTICULATIONS, BONES)}

Therapeutic Methods.-The diseases of the organs of locomotion comprise the principal part of surgery. They include the consideration of inflammation, atrophy, paralysis and laceration of the muscles, paralysis of the nerves, inflammation and laceration of tendons, inflammation and fracture of the bones, and inflammation and dislocation of the articulations. The therapeutic methods employed in the treatment of these diseases are naturally very numerous and differ with the character of the individual case. With regard to them and the excellent natural healing processes which operate in these conditions, especially in bone fractures, the reader is referred to the text-books on general surgery. Nevertheless, there are some observations to be made from the standpoint of general therapeutics. The surgical methods of treatment most frequently used are the following:

1. The rest treatment is the most important method of healing in all painful and acute inflammatory conditions of the muscles, tendons, bones, and articulations. "Rest the afflicted part." Simply permitting horses to "stand" will alone in many cases bring about healing (natural healing). The application of a plaster-of-Paris dressing in fracture of bones and sprained joints acts in the same manner.

2. The exercise treatment, on the contrary, is indicated in some chronic inflammatory conditions of the muscles, nerves, tendons and articulations (muscular rheumatism, atrophy of muscles, paralyses of muscles and nerves, and contractions). By exercise, as by massage, the circulation, resorption, innervation and metabolism are stimulated locally and generally, muscular activity and the body constitution are strengthened, and under 288 
certain circumstances pain is decreased. In addition, exercise operates as a prophylactic against hæmoglobinæmia [azoturia], parturient apoplexy and stable founder.

3. Hydrotherapy (cold, heat, Priessnitz dressing), cutaneous irritants, firing, massage, and electrotherapy are very important methods of treatment in numerous acute and chronic surgical disease conditions. (See the chapters on those methods.)

4. The medical method consists in the use of direct stimulants of the muscles and nerves: veratrin and strychnine; also in the subcutaneous and parenchymatous injection of morphine, cocaine, sodium chloride, and oil of turpentine (shoulder lameness).

5. The operative treatment is either direct or indirect. Examples of the direct method are: section of contracted tendons or muscles (tenotomy, myotomy), incision of purulent inflammations of muscles, bones and tendon sheaths, and resection of necrotic tendons, while neurotomy is an example of the indirect or symptomatic method.

6. Regulation of the shoeing is very important in numerous surgical diseases (diseases of the hoof, ring bone, spavin, diseases of tendons). 


\section{INDIFFERENT REMEDIES. MECHANICALS}

Definition.-The terms indifferent and mechanical are used to designate a group of therapeutic agents which in general do not produce any chemical or pharmacological effect upon the organism and which are employed only for their mechanical action. The group includes protective, emollient, cleansing, absorbent, dilating, and uniting remedies.

\section{PROTECTIVES}

Synonyms: Obtegents, involvents, obvolents, lubricants; covering, protecting, soothing remedies, bandages or dressings.

Uses.-The protectives serve to cover the skin and mucous membranes in inflammatory conditions and when wounds are present; also to guard and defend these structures against injurious influences. Wounds upon the skin are most frequently treated by the use of bandaging materials. In addition, the different varieties of eczema, erosions and burns and also the specific inflammations of the skin require the employment of protective remedies in the form of salves. The latter are also applied to the skin prophylactically to protect it from flowing secretions (pus) and caustic substances (cantharides). The protectives are administered internally in the different inflammatory conditions of the mucous membrane of the pharynx, cesophagus, stomach and intestines and as mechanical antidotes in intoxications (see p. 216); the mucous membrane of the bladder and rectum is also accessible to applications of protectives. Finally, they are used to prevent the dissipation of heat and moisture from the skin, to promote cutaneous resorption in the epidermatic application of medicines and to lubricate the instruments and hands.

Remedies.-1. Fats and oils: Lard, olive oil, linseed oil, peanut oil, almond oil, rape oil, poppy-seed oil, cod liver oil, cacao oil, 290 
coca oil, mutton suet, spermaceti, ointment of rose water, and sevum salicylatum. ${ }^{1}$

2. Mucilaginous remedies: Linseed, acacia, althæa, tragacanth, salep, mallow, dietetic remedies (oat and barley mucilage).

3. Paraffin ointment, ${ }^{2}$ lanolin, simple cerate.

4. Plasters and liniments: Adhesive plaster, Lund's plaster, ${ }^{3}$ Carron oil (linamentum calcis).

5. Silver nitrate (silver covering of burns and other cutaneous affections).

6. Gelatin, collodion, traumaticin [gutta-percha dissolved in chloroform], water glass.

7. Starch, lycopodium, zinc oxide, bolus, talcum, pulvis talci salicylicus (N.F.).

8. Cotton and other dressing material,

\section{EMOLLIENTS}

Synonyms: Demulcents, solvents; softening, loosening, liquefying, disintegrating remedies.

Actions and Uses.-The emollients operate upon the different pathological indurations of the skin, subcutis, tendons, tendon sheaths, and muscles by mechanical diffusion and imbibition (water, oil, glycerin, soap), by preventing the dissipation of heat and water (poultices) and by chemical solution (lyes, alkalies), disintegration and liquefaction. The remedies concerned in internal softening and solution have already been considered in the chapters on resorbents (p. 76), expectorants (p. 107) and diuretics (p. 113.) The external disease conditions which are most frequently treated with emollients are thickenings of the skin, calluses, scabs and crusts upon the skin, squamous eczema, thickenings of the subcutis, hard swellings in the tendons and tendon sheaths as in

[1 Salicylic acid 2 parts, benzoic acid 1, mutton suet 97.]

[2 Petrolatum album.]

[ 3 Turpentine and pitch, equal parts; liquefy by heat, mix, and spread on cloth.] 
tendinitis and tendovaginitis, indurations of glands, udder nodules and rheumatic indurations of muscles.

Remedies.-1. Water and all remedies containing water (baths, cataplasms, moist applications).

2. Moist heat, especially in the form of the Priessnitz dressing and cataplasms.

3. Fats, soaps, glycerin, and lyes (soda lye, potash lye, potassium sulphate).

\section{CLEANSING REMEDIES}

Action and Uses.-The ordinary cleansing agents, water and soap, operate mechanically to remove dirt, scabs, pus, and other disease products, and particularly infectious materials. In the latter respect, the cleansing agents act at the same time as disinfectants. As already stated (p. 182), a thorough cleansing is in many cases equal in value to a disinfection. Water and soap, when rationally used, frequently suffice as disinfectants; provided the water is clean, i.e., free from infectious materials, that it is used in sufficient amount and, when possible, warm or hot, and that a good preparation of soap is used. An entirely neutral soap is best for cleansing the skin (soft soap irritates the skin, as may be frequently observed especially in dogs). On the other hand, for cleansing utensils and other articles a soap with a strong alkaline reaction is desired, i.e., a soap which contains free lye, because the free lye exerts a disinfectant as well as a cleansing effect. Soft soap is therefore preferred for this purpose.

Absorbent Remedies (Imbibents, Rophetics).-These serve to absorb blood, serum, pus, and other fluids. Their action is purely physical. They include dressing materials (cotton, wood wool, jute, etc.). Wood wool possesses the greatest imbibing properties. Freshly-burned charcoal absorbs gases $\left(\mathrm{SH}_{2}\right)$ and poisons, and starch absorbs iodine.

Dilating Remedies (Dilatants).-A mechanical dilation by means of dilating pencils (laminaria, tupelo) is used in surgery and obstetrics. 
Uniting Remedies (Contentives). - These serve to unite solutions of continuity (wounds, fractures of bones). The so-called immovable dressings are made of rubber, gelatin, water glass, plaster-of-Paris, starch paste, etc. Medical uniting substances for wounds are collodion, traumaticin [gutta-percha dissolved in chloroform], and adhesive plaster. 


\section{AIR AS A REMEDY}

General.-While the air acts as the excitant or carrier in different diseases, it is also an important healing factor in a number of diseases. As regards the harmful effects of the air, these can be referred in part to its temperature and in part to the admixture of dust, bacteria, and poisons. Stable air is an especially frequent source of diseases of all kinds. Its constant, uniform temperature, which is usually too high, exerts a relaxing effect upon the general body constitution and produces a consequent predisposition to disease. The best known of the stable diseases which occur through the influence of the temperature of the stable air are rheumatism, rheumatic hæmoglobinæmia [azoturia], catarrhs, brain and lung diseases, and also summer wounds in army horses. Secondly, stable air operates pathologically through its contained bacteria, gases and mechanical admixtures. These favor especially the development of tuberculosis, glanders, contagious pneumonia of horses and other infectious diseases. The carbon dioxide, ammonia and other gases which may be present act harmfully upon the respiration. The outside air can also cause disease in different ways (hot air, dusty air, arsenical fumes, lead vapors). By a careful prophylaxis all of these disease conditions may be indirectly avoided.

Furthermore, air is a very important direct curative agent. Its healing action is in part due to the oxygen it contains (open suppuration, anaërobes), in part to its temperature and in part to its purity. To what extent the ozone of the air possesses healing properties has not been scientifically determined.

Therapeutic Action of Cold Air.-Cold air operates first, like cold water, as a cutaneous irritant. The anæmia produced primarily in the cutaneous vessels is followed by a reactive hyperæmia of the skin. As a consequence the circulation is stimulated through294 
out the entire body and the blood is driven from the internal organs, especially the lungs, digestive apparatus and brain, to the periphery, metabolism is increased and the constitution is strengthened. Heat is given off by the body to the surrounding cold air, provided the action of the latter continues long enough. Cold air consequently acts as a refrigerant. Upon the basis of these actions, cold air is employed in the form of ventilation of stables, and animals are exercised in cold air, bivouacked and placed in cold compartments and in shady places to make them hardy; cold air is also employed as a preventive against rheumatism and other diseases due to chilling, as a curative agent in all febrile diseases of the internal organs, and in congestion of the lungs and brain.

Therapeutic Action of Pure Air.-Pure air, rich in oxygen, poor in carbon dioxide, free from bacteria and other impurities, is the best natural remedy in all catarrhal affections of the respiratory apparatus. It is employed in the form of good ventilation, the air in the stable being renewed as often as possible, or the animal is placed in the open air (pasture, bivouac, exercise in the open). The renewal of the air in the bronchi and alveoli promotes respiration and removes the injurious air from these structures. Furthermore, the introduction of fresh air dilutes and removes the infectious material present, which is also in part destroyed by the oxygen (disinfectant action of fresh air). Especially good results have been obtained in this respect in strangles, contagious pneumonia, tuberculosis, and other infectious diseases.

Climate.-Climate can only be made use of for healing purposes in veterinary medicine in exceptional cases, which is in contrast to the very highlydeveloped climato-therapy of human medicine. The importance of climate in a hygienic sense for the domestic animals has, it is true, been known for a long time. It is known, for example, that the mountain breeds, because of the vigorous climate, are more resistant to diseases of all kinds than the breeds of the lowlands. It is further known that imported animals (monkeys, parrots) are very susceptible to disease and frequently die of tuberculosis because of the change of climate. A similar effect is observed on horses, cattle and sheep when they are taken into $\mathrm{a}$ new region with a different climate. 
For economical reasons, a change of climate for therapeutic purposes is permissible with the domestic animals only in very rare cases (removal to elevated pastures or to distant farms), although frequently such a change appears to be indicated, especially in convalescence.

Light.-The use of light as a healing remedy was introduced by Finsen of Copenhagen. He uses the bactericidal (chemical action of the ultraviolet, violet and blue rays) as well as the inflammation-producing (thermal action of the red rays) forces of light and has in this manner cured numerous cases of lupus in man. His method consists in concentrating the rays of a very strong arc light by means of a rock crystal and directing them through a layer of distilled water which is continually cooled. Iwanow (Repert. d. pol. Sanit. vét., 1902) has obtained excellent results with Finsen's apparatus in the treatment of eczemas of the horse.

Röntgen's Rays.-These are used in surgery for the local treatment of malignant neoplasms (carcinomas, sarcomas). Radium acts in a similar manner (radiotherapy). In internal medicine, the Röntgen rays have proven less satisfactory (employed in chronic myelomas and in lymphatic leukæmia and aleukamic splenic tumors). 


\section{INDEX}

Abortive method, 8

Abortives, 119

Absorbent materials, 292

Absorbents of gas, 32, 52

Acid-neutralizing stomachice, 32

Acrics, 146

Air as a remedy, 104, 294

harmful effects of, 294

Alactics, 127

Alterants, 76, 159

Amara, 30

Amblotics, 119

Anæsthesia, general and local, 101, 102

Anæsthetics, 97

Analeptics, 93

Analgesics, 97

Anaphrodisiacs, 121

Anexosmotics, 48

Angio-asthenics, 72

Angiosthenics, 71

Anodynes, 97

Antacids, 32

Antagonisties, 216

Antagonists, 216

Antaphrodisiacs, 121

Antatrophics, 134

Antemetics, 32

Antentozoa, 165

Antepizon, 160

Anterotics, 121

Anthelmintics, 165

Anti-asthmatics, 97

Anticatarrhalics, 159

Anticathartics, 48

Anticonvulsives, 97

Antidepertitorics, 134

Antidiarrhoics, 48

Antidotes, 216

chemical, 217

mechanical, 216

physiological, 219

symptomatic, 220

Antidyscratics, 159

Antidysenterics, 48

Antidyspeptics, 29

Antiepileptics, 97

Antifebrilics, 84

Antifermentatives, 169

Antiferments, 52

Antigalactagogues, 127

Antigalactics, 127
Antihidrotics, 132

Antineuralgics, 97

Antiparalytics, 93

Antiparasitics, 109, 160

Antipediculous, 160

Antiperiodics, 84

Antiphlogistics, 159

Antiphthiriacs, 160

Antiplastics, 76, 138

Antipsorics, 160

Antiputrids, 169

Antipyretics, 84, 86

Antiscabious, 160

Antisepsis, 170

Antiseptic stomachics, 31 styptics, 49

Antiseptics, 109, 169, 172 for wounds, 209 internal, 213

Antisialagogues, 132

Antispasmodics, 52, 97

Antitænics, 165

Antitetanics, 97

Antithermics, 84

Antitoxics, 216

Antitypics, 84

Antityposics, 84

Antizymotics, 169

Antodontalgics, 97

Aperients, 40

Aperitives, 40

Aphrodisiacs, 120

Arabian medicine, 14

Aristotle, 12

Aromatic stomachics, 31

Artificial arrest of hemorrhage, 68

Asclepiadæ, 9

Asepsis, 170

Aseptics, 169

Astringent styptics, 49

Astringents, 48, 157

Attenuation, methods of, 230

Balneotherapy, 265

Bechics, 109

Bitter stomachies, 30

Bleeding, 286

Blisters, 146

Blood diseases, dietetic treatment of, 68

general therapeutics of, 60 
Blood-forming remedies, 63

Blood plastics, 63,134

Blood-vessels, general therapeutics of the diseases of, 64

Boerhaave, 10, 17

Bone plastics, 134

Bradysphygmics, 57

Brown, 18

Calefacients, 159

Cardiac diseases, general therapeutics of, 56

sedatives, 58

stimulants, 58

tonics, 58

Cardiacs, 57

Carminatives, 52

Cathæretics, 152

Catharsis, results of, 43 theories of, 41

Cathartics, 40, 45

Causal healing method, 6

Causes of immunity, 227

Caustics, 152

Cauterics, 152

Cauterization, 156

Cellular pathology, 21

Cerebralics, 91

Chemical antidotes, 217

Chemotherapy, 23

Cholagogue laxatives, 54

Cholagogues, 54

Cleansing remedies, 292

Climate, 295

Coagulants, 157

Cold, 69

Colyseptics, 169, 172

Composting, 202

Compression, 68, 276

Confortants, 137

Confortatives, 137

Conservants, 169

Conservation of animal products, 214

Conservative healing method, 8

Constipating remedies, 48

Contentives, 293

Corrosives, 152

Curative vaccination, 252

Cutaneous irritants, 146 mallein test, 263 tuberculin test, 260

Demulcent styptics, 48

Demulcents, 291

Derivants, 146

Derivative healing method, 5 medicines, 146
Dermerethistics, 146

Diagnostic inoculation, 254

Diaphoretics, 129

Diapnoics, 129

Dietetic method, 7 treatment of blood diseases, 63 intestinal diseases, 40 liver diseases, 55 stomach diseases, 29

Digestive organs, general therapeutics of, 25

Digestives, 29

Dilatants, 292

Dilating remedies, 292

Direct healing method, 5

Discutients, 76

Disinfectant stomachics, 31

Disinfectants, 169 for infectious diseases, 201

for wounds, 209

mechanical, 208

relative rank of, 173

Disinfection for infectious animal diseases, 183

of wounds, 178, 209

preliminary, 182 preparation for, 182

Diuretics, 113

Drastics, 40

Ebriantics, 97

Ecbolics, 119

Eccoprotics, 40

Ectrotics, 119

Electropuncture, 282

Electricity as a remedy, 282

Electrolysis, 282

Emesis, effects of, 35

Emetics, 34, 216

Emmenagogues, 119

Emollients, 291

Empirical healing method, 7

Enterostyptics, 48

Epileptifacients, 93

Epispastics, 146

Erasistratus, 12

Erethistics, 146

Erotics, 120

Errhines, 107

Erythrotics, 63

Escharotics, 152

Euphorics, 93

Euplastics, 63, 134

Evacuants, 40

Excitants, 93

Exhilarants, 93

Expectant healing method, 7 
Expectorants, 107

Exsiccants, 157

External antiparasitics, 160

Frudates, general therapeutics of, $\mathbf{7 3}$

Eye diseases, general therapeutics of, 141

\section{Fallopius, 16}

Faradotherapy, 282

Febrifuges, 84

Fever, general therapeutics of, 80

Firing, 156

Foods, 134

Franklinotherapy, 282

Galactagogues, 122

Galactics; 122

Galen, 12

Galvanocaustic, 282

Galvanotherapy, 282

Gas absorbents, 52

Gas-expelling drugs, 52

General healing method, 5

Genital organs, general therapeutics of the diseases of, 118

Glands, general therapeutics of, 128

Hæmatics, 63

Hæmatinics, 63

Hæmatopoietics, 63

Hæmostatics, 69

Hahnemann, 19

Harvey, 16

Healing methods, 4

Heart diseases, general therapeutica of, 56

remedies, 57

sedatives, 58

stimulants, 57 tonics, 58

Heat, 69

Hemorrhage, methods of arresting, 63

Hepatic stimulants, 54

Hepatics, 54

Herophilus, 12

Hidrotics, 129

Hippocrates, 10

History of therapeutics, 9

Hoffmann, 17

Homceopathy, 19

Hufeland, 18

Humoral pathology, 11, 13

Hydragogue catharties, 43 diuretics, 113

Hydriatrics, 265

Hydrotherapy, 105, 265

Hyperæmia as a remedy, 271
Hyperinotics, 63

Hyperkineties, 93

Hypnotics, 97

Imbibents, 292

Immunity, 225

acquired, 226

active, 226

artificial, 227

causes of, 227

individual, 225

inherited, 226

natural, 225

passive, 226

species, 225

Indifferent remedies, 290

Indirect healing method, 5

Inhalations, 105, 106

Inoculation, diagnostic, 254

methods of, 225

Internal antiseptics, 213

Intestinal diseases, dietetic treatment of, 40 general therapeutics of, 38 mechanical treatment of, 51 operative treatment of, 51

Intracutaneous tuberculin test, 260

Intradermal tuberculin test, 260

Involvents, 290

Irritants, 146

Kneading, 276

Lactics, 122

Lactifuges, 127

Laxatives, 41

Lenitives, 41

Ligation, 68

Light, 208, 296

Litholytics, 113

Lithothryptics, 113

Liver diseases, dietetic treatment of, 55

general therapeutics of, 52 mechanical treatment of, 55 remedies, 53

Locomotory organs, general therapeutics of, 288

Lubricants, 290

Mallein, 260

test, cutaneous, 263

ophthalmic, 260, 263

subcutaneous, 261

Malpighi, 16

Mange remedies, 160

Massage, 276 
Masticatives, 131

Masticatorics, 131

Mechanical antidotes, 216

disinfectants, 208

treatment of intestinal diseases, 51

of liver diseases, $\mathbf{5 5}$ of stomach diseases, 33

Mechanicals, 290

Mechanotherapy, 276

Medicinal plastics, 135

Metabolism, general therapeutics of the diseases of, 133

Metasyncritics, 159

Methods of healing, 4

Mitigation, methods of, 230

Monk's medicine, 14

Morgagni, 16

Mucous membranes, general therapeutics of, 143

Mucus-dissolving remedies, 109

Mydriatics, 141

Myotics, 142

Narcosis, 101

Narcotic stomachics, 32 styptics, 49

Narcotics, 97

Nauseo6a, 34

Nauseotics, 34

Nerve sedatives, 97 stimulants, 93

Nervines, 90

Nervous system, general therapeutics of diseases of, 88

Neurotics, 90

Nutrients, 134, 137

Obstruents, 48

Obtegents, 290

Obvolvents, 290

Odinegogues, 119

Operative treatment of intestinal diseases, 51

of stomach diseases, 34

Ophthalmic mallein test, 260, 263 tuberculin test, 259

Osmotics, 40

Palliative method, 6

Paracelsus, 9, 14

Paralyzants, 97

Parasitics, external, 160

Paré, 16

Paregorics, 97

Parturefacients, 119

Pasteurization, 207
Pellentics, 119

Peptics, 29

Peripherics, 92

Peristal tics, 40

Phlebotomy, 286

Physical antidotes, 216

Physiological antidotes, 219 healing method, 6 stomachics, 29

Plastics, 134 food, 134 medicinal, 134

Pneumatics, 107

Polysphygmics, 57

Preservants, 169

Preservation of animal products, 214

Priessnitz, 19, 265

Priessnitz's dressing, 273

Prophylactic method, 7

Protective vaccination, 236

Protectives, 48, 290

Ptarmics, 107

Ptyalogogues, 131

Pupil-contracting remedies, 142

Pupil-dilating remedies, 141

Purgatives, 40

Pustulants, 146, 148

Rademacher, 19

Radical healing method, 6

Rational healing method, 6

Reducing remedies, 138

Refrigerants, 159

Regulations concerning vaccination, 237

for disinfection, 184

Resolvents, 76

Resorbents, 76

Respiratory apparatus, general therapeutics of the diseases of, 103

Roborants, 137

Röntgen's rays, 296

Rophetics, 292

Rubbing, 276

Rubefacients, 146, 148

Ruminatorics, 32

Saline stomachics, 30

Schönlein, 18

Sedantics, 97

Sedatives, cardiac, 58 nerve, 97

Serum therapy, 22

Sialagogues, 131

Sialics, 131

Skin, general therapeutics of, 143, 144

Solvents, 291 
Somniferics, 97

Soporifics, 97

Spasmodics, 93

Spinalics, 92

Spinants, 93

Spontaneous arrest of hemorrhage, 66

Stahl, 18

Statistical healing method, 7

Sternutatorics, 107

Stimulant stomachics, 31

Stimulants, cardiac, 58 nerve, 93

Stomach diserses, dietetic treatment of, 29 general therapeutics of, 25 mechanical treatment of, 33

Stomachics, 29 operative treatment of, 34

Stroking, 276

Styptics, general, 70 intestinal, 48 local or topical, 69

Subeutaneous mallein test, 261 tuberculin test, 258

Sudorifics, 129

Suppletives, 29

Suppurants, 148

Suppuratives, 146

Sydenham, 17

Symptomatic antidotes, 220 healing method, 6

Tapping, 276

Temperantics, 97

Temperants, 159

Tetanics, 93

Thermopenetration, 285

Tonics, 137, 157 cardiac, 58

Torsion, 68

Transfusion of blood, 62

Transudates, general therapeutics of, 73
Tuberculin, 258

test, cutaneous and intracutaneous, 260

ophthalmic, 259

subcutaneous, 258

Udder, general therapeutics of the diseases of, 118

Uniting remedies, 293

Urinary organs, general therapeutics of the diseases of, 112

Uterines, 119

Uterus, general therapeuties of the diseases of, 118

Vaccination, 225, 250

curative, 252

emergency, 236

for the different diseases, 237

methods of, 233

prophylactic, 236

protective, 236

purposes of, 235

varieties of, 235

Vaso-astringents, 71

Vaso-constringents, 71

Vasodilants, 72

Vasodilators, 72

Vasomotor stimulante, 71

Venesection, 286

Vermifuges, 165

Vesal, 16

Vesicants, 146, 148

Vibration, 276

Vital healing method, 8

Vomiting, effects of, 35

Vomitives, 34

Vomitorics, 34

Von Haller, 18

Waiting healing method, 7

Water as a remedy, 265

Worm remedies, 165

Wound disinfectants, 209 








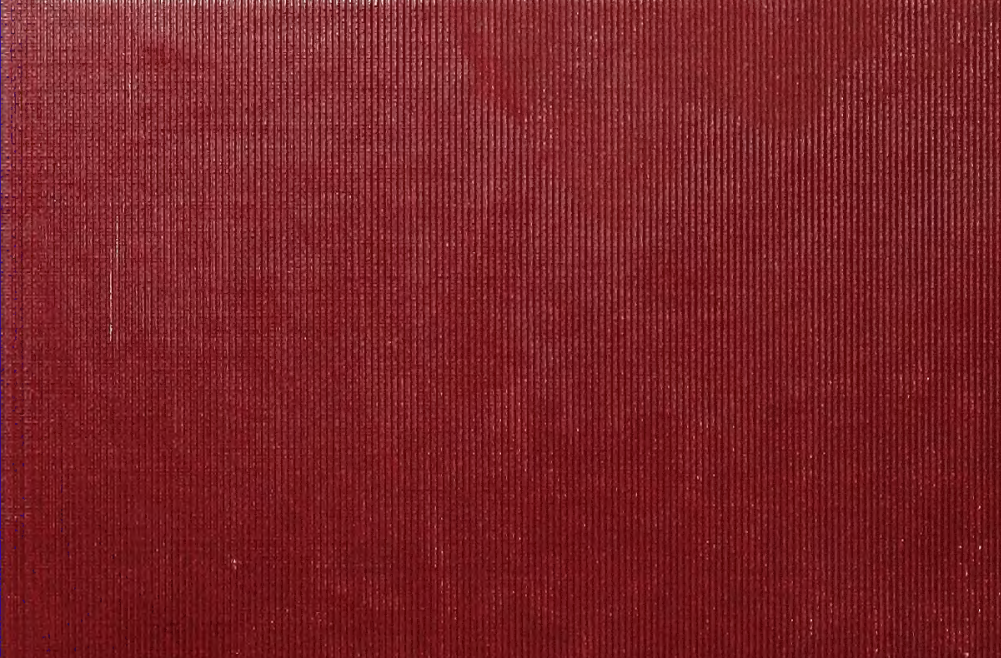

